University of Tennessee Health Science Center

UTHSC Digital Commons

\title{
Adverse Effects of Asparaginase in Pediatric Patients with Acute Lymphoblastic Leukemia
}

Chengcheng Liu

University of Tennessee Health Science Center

Follow this and additional works at: https://dc.uthsc.edu/dissertations

Part of the Diseases Commons, Medical Sciences Commons, and the Therapeutics Commons

\section{Recommended Citation}

Liu, Chengcheng , "Adverse Effects of Asparaginase in Pediatric Patients with Acute Lymphoblastic Leukemia" (2015). Theses and Dissertations (ETD). Paper 157. http://dx.doi.org/10.21007/ etd.cghs.2015.0185.

This Dissertation is brought to you for free and open access by the College of Graduate Health Sciences at UTHSC Digital Commons. It has been accepted for inclusion in Theses and Dissertations (ETD) by an authorized administrator of UTHSC Digital Commons. For more information, please contact jwelch30@uthsc.edu. 


\title{
Adverse Effects of Asparaginase in Pediatric Patients with Acute Lymphoblastic Leukemia
}

\begin{abstract}
Acute lymphoblastic leukemia (ALL) is the most common type of childhood cancer. Asparaginase is a critical treatment component for ALL. However, its use is complicated by adverse effects, such as hypersensitivity, osteonecrosis and pancreatitis.

Hypersensitivity to asparaginase typically requires discontinuation of current formulation and substitution with other formulations, but the differential diagnosis can be challenging, and the diagnostic utility of antibody tests is unclear. We comprehensively analyzed anti-Elspar (native E.coli asparaginase) IgG antibodies in 410 pediatric patients treated on an asparaginaseintensive front-line clinical trial. Of 169 patients (41.2\%) who exhibited clinical allergy, 147 (87.0\%) were positive for anti-Elspar antibody. Of 241 patients without clinical allergy, $89(36.9 \%)$ had detectable antibody. Among those positive for antibody, the antibody titers were higher in those who developed allergy than in those who did not $(P<1.0 \times 10-15)$. Antibody measures at week 7 of continuation therapy had a sensitivity of $87 \%-88 \%$ and a specificity of $68 \%-69 \%$ for predicting or confirming clinical reactions. Antibodies were inversely associated with serum asparaginase activity $(P=7.0 \times 10-6)$. Interestingly, high antibodies were associated with a lower risk of osteonecrosis (odds ratio $=0.83 ; 95 \%$ confidence interval, $0.78-0.89 ; \mathrm{P}=0.007$ ), which is a dose-limiting adverse effect of glucocorticoids but has also been linked to asparaginase treatment. We conclude that antibodies were related to clinical allergy and to low systemic exposure to asparaginase, leading to lower risk of other adverse effects of therapy. Measures of serum antibodies to asparaginase can be useful in patients with ALL.

Osteonecrosis is a common dose-limiting toxicity of glucocorticoids. Data from clinical trials suggest that other medications can increase the risk of glucocorticoid-induced osteonecrosis. Here we utilized a mouse model to study the effect of asparaginase treatment on dexamethasone-induced osteonecrosis. After 6 weeks of treatment, mice receiving asparaginase along with dexamethasone had a higher rate of osteonecrosis than those receiving only dexamethasone ( $44 \%$ vs. $10 \%, P=0.006)$. Primary epiphyseal arteriopathy, an initiating event for osteonecrosis, was observed in $58 \%$ of mice receiving asparaginase and dexamethasone compared to $17 \%$ of mice receiving dexamethasone only $(P=0.007)$. As in the clinic, greater exposure to asparaginase was associated with greater plasma exposure to dexamethasone $(\mathrm{P}=$ 0.0001). This model also recapitulated other clinical risk factors for osteonecrosis, including age at start of treatment, and association with the systemic exposure to dexamethasone $(P=0.027)$ and asparaginase $(P=0.036)$. We conclude that asparaginase can potentiate the osteonecrotic effect of glucocorticoids.
\end{abstract}

Acute pancreatitis is a serious complication of asparaginase with no definitive treatment. Risk factors for asparaginase-induced pancreatitis, especially the genetic predisposition, have not been clearly identified. We studied 5398 pediatric patients with ALL and showed that older age, higher exposure to asparaginase, higher Native American ancestry or African ancestry were independent clinical risk factors for pancreatitis. To determine genetic risk factors, we performed a genome-wide association study. A rare nonsense variant rs199695765 in CPA2, a pancreatic enzyme, was highly associated with pancreatitis (odds ratio $=588,95 \%$ confidence interval 66.8- 5166, $\mathrm{P}=9.0 \times 10-9$ ). A gene-level analysis showed an excess of additional CPA2 variants in those who did versus did not develop pancreatitis $(P=0.018)$. Furthermore, common variants in genes critical to purine metabolism and cytoskeleton function were also associated with development of pancreatitis. Our findings are consistent with a mixed genetic architecture underlying serious adverse drug effects, wherein a combination of rare but highly penetrant and common but weakly penetrant genetic risk factors contribute to genetic risk. For the patients carrying the highly penetrant variants, consideration should be given to treatment with a nonasparaginase 
containing ALL chemotherapy regimens.

Overall, we studied the major adverse effects of asparaginase in patients treated for ALL. These findings will provide important guidance for precision medicine.

\section{Document Type}

Dissertation

\section{Degree Name}

Doctor of Philosophy (PhD)

\section{Program}

Biomedical Sciences

\section{Research Advisor}

Mary V. Relling, Pharm.D.

\section{Keywords}

Acute lymphoblastic leukemia Asparaginase, GWAS, Hypersensitivity, Osteonecrosis, Pancreatitis

\section{Subject Categories}

Diseases | Medical Sciences | Medicine and Health Sciences | Therapeutics 


\title{
ADVERSE EFFECTS OF ASPARAGINASE IN PEDIATRIC PATIENTS WITH ACUTE LYMPHOBLASTIC LEUKEMIA
}

\author{
A Dissertation \\ Presented for \\ The Graduate Studies Council \\ The University of Tennessee \\ Health Science Center
}

\begin{abstract}
In Partial Fulfillment
Of the Requirements for the Degree

Doctor of Philosophy

From The University of Tennessee
\end{abstract}

By

Chengcheng Liu

August 2015 
Copyright (C) 2015 by Chengcheng Liu. All rights reserved. 


\section{DEDICATION}

This work is dedicated to my parents

Haiying Liu

Hua Cheng

and my husband

$\mathrm{Hao} W u$

Thank you for all of your love and support along the way. 


\section{ACKNOWLEDGEMENTS}

First and foremost, I would like to express my most sincere gratitude and appreciation to my advisor, Dr. Mary V. Relling, who has been extremely supportive to me over these years. Her insight, guidance and commitment to the highest standards have deeply inspired and motivated me. Despite her busy schedules, she was always there to guide my research, clarify my doubts, help me with writing, and encourage me to believe in my abilities. I simply cannot imagine having completed the Ph.D. study with a better advisor.

I really appreciate the guidance, support and encouragement of my committee members, Dr. Laura Janke, Dr. Cheng Cheng, Dr. Jun Yang, Dr. David Nelson, and Dr. Russell Chesney - sadly he will not see me graduate. I would particularly like to acknowledge the important contribution of Dr. Janke to our animal research projects.

I am grateful to all the present and past members of the lab, Dr. Laura Ramsey, Dr. Christian Fernandez, Dr. Seth Karol, Dr. Jean Cai, Dr. Colton Smith, David Jenkins, Monique Payton, Pamela McGill, Dr. Jitesh Kawedia and Dr. Tamara Chang. It has been the most pleasurable experience working in the lab with a group of talented scientists as well as caring friends. Dr. Wenjian Yang deserves a special acknowledgement for sharing his invaluable expertise and guidance in computational genomics. I would like to thank Deqing Pei for her assistance with statistical analysis, and Dr. William Evans for his scientific insight and helpful advice.

Thanks to University of Tennessee Health Science Center and St. Jude Children's Research Hospital for providing me with an excellent environment for doing research. I am deeply indebted to everyone who participated, helped and supported my research projects directly or indirectly. Finally, I would like to thank my parents Haiying Liu and Hua Cheng, and my husband Hao Wu, who also graduated from UTHSC. I could never have completed this work without your love and support. 


\begin{abstract}
Acute lymphoblastic leukemia (ALL) is the most common type of childhood cancer. Asparaginase is a critical treatment component for ALL. However, its use is complicated by adverse effects, such as hypersensitivity, osteonecrosis and pancreatitis.

Hypersensitivity to asparaginase typically requires discontinuation of current formulation and substitution with other formulations, but the differential diagnosis can be challenging, and the diagnostic utility of antibody tests is unclear. We comprehensively analyzed anti-Elspar (native E.coli asparaginase) IgG antibodies in 410 pediatric patients treated on an asparaginase-intensive front-line clinical trial. Of 169 patients $(41.2 \%)$ who exhibited clinical allergy, 147 (87.0\%) were positive for anti-Elspar antibody. Of 241 patients without clinical allergy, $89(36.9 \%)$ had detectable antibody. Among those positive for antibody, the antibody titers were higher in those who developed allergy than in those who did not $\left(P<1.0 \times 10^{-15}\right)$. Antibody measures at week 7 of continuation therapy had a sensitivity of $87 \%-88 \%$ and a specificity of $68 \%-69 \%$ for predicting or confirming clinical reactions. Antibodies were inversely associated with serum asparaginase activity $\left(P=7.0 \times 10^{-6}\right)$. Interestingly, high antibodies were associated with a lower risk of osteonecrosis (odds ratio $=0.83 ; 95 \%$ confidence interval, $0.78-0.89 ; P=$ 0.007), which is a dose-limiting adverse effect of glucocorticoids but has also been linked to asparaginase treatment. We conclude that antibodies were related to clinical allergy and to low systemic exposure to asparaginase, leading to lower risk of other adverse effects of therapy. Measures of serum antibodies to asparaginase can be useful in patients with ALL.
\end{abstract}

Osteonecrosis is a common dose-limiting toxicity of glucocorticoids. Data from clinical trials suggest that other medications can increase the risk of glucocorticoidinduced osteonecrosis. Here we utilized a mouse model to study the effect of asparaginase treatment on dexamethasone-induced osteonecrosis. After 6 weeks of treatment, mice receiving asparaginase along with dexamethasone had a higher rate of osteonecrosis than those receiving only dexamethasone ( $44 \%$ vs. $10 \%, P=0.006)$. Primary epiphyseal arteriopathy, an initiating event for osteonecrosis, was observed in $58 \%$ of mice receiving asparaginase and dexamethasone compared to $17 \%$ of mice receiving dexamethasone only $(P=0.007)$. As in the clinic, greater exposure to asparaginase was associated with greater plasma exposure to dexamethasone $(P=$ 0.0001). This model also recapitulated other clinical risk factors for osteonecrosis, including age at start of treatment, and association with the systemic exposure to dexamethasone $(P=0.027)$ and asparaginase $(P=0.036)$. We conclude that asparaginase can potentiate the osteonecrotic effect of glucocorticoids.

Acute pancreatitis is a serious complication of asparaginase with no definitive treatment. Risk factors for asparaginase-induced pancreatitis, especially the genetic predisposition, have not been clearly identified. We studied 5398 pediatric patients with ALL and showed that older age, higher exposure to asparaginase, higher Native American ancestry or African ancestry were independent clinical risk factors for 
pancreatitis. To determine genetic risk factors, we performed a genome-wide association study. A rare nonsense variant rs199695765 in $C P A 2$, a pancreatic enzyme, was highly associated with pancreatitis (odds ratio $=588,95 \%$ confidence interval 66.8-5166, $P=$ $\left.9.0 \times 10^{-9}\right)$. A gene-level analysis showed an excess of additional CPA2 variants in those who did versus did not develop pancreatitis $(P=0.018)$. Furthermore, common variants in genes critical to purine metabolism and cytoskeleton function were also associated with development of pancreatitis. Our findings are consistent with a mixed genetic architecture underlying serious adverse drug effects, wherein a combination of rare but highly penetrant and common but weakly penetrant genetic risk factors contribute to genetic risk. For the patients carrying the highly penetrant variants, consideration should be given to treatment with a non-asparaginase containing ALL chemotherapy regimens.

Overall, we studied the major adverse effects of asparaginase in patients treated for ALL. These findings will provide important guidance for precision medicine. 


\section{TABLE OF CONTENTS}

CHAPTER 1. BACKGROUND....................................................................................

Acute Lymphoblastic Leukemia (ALL) ............................................................ 1

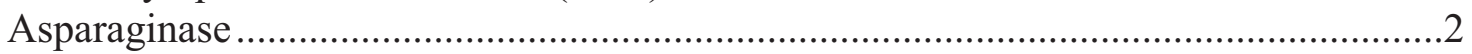

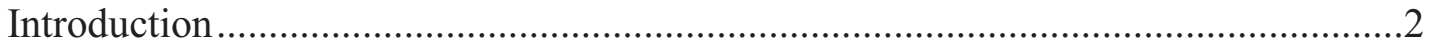

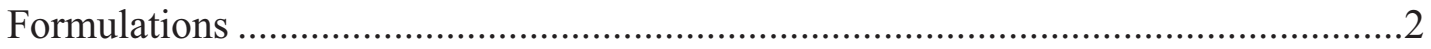

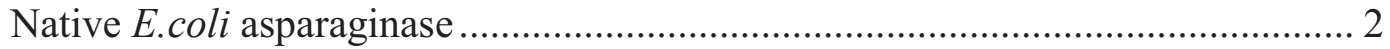

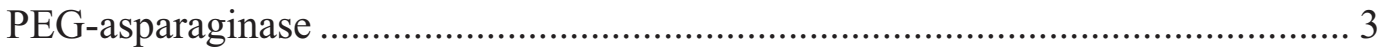

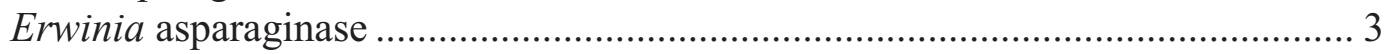

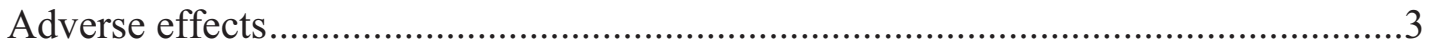

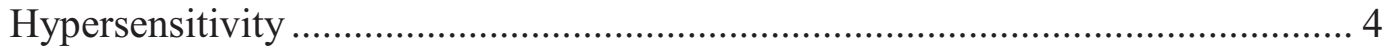

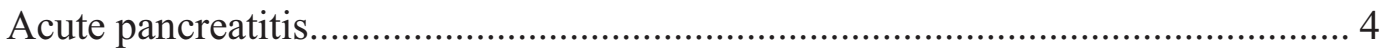

Drug interaction with glucocorticoids ...................................................... 6

Effect on glucocorticoid-induced osteonecrosis ........................................... 6

Pharmacogenomics: A Brief Introduction .......................................................

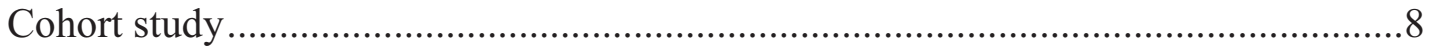

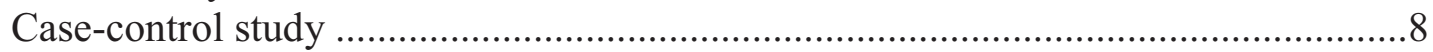

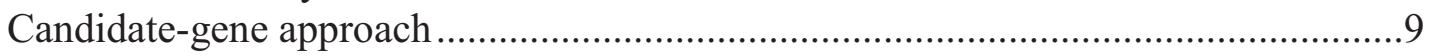

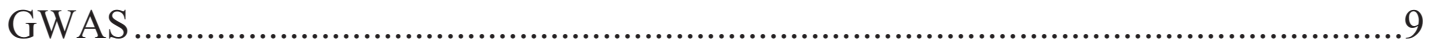

CHAPTER 2. ASPARAGINASE HYPERSENSITIVITY.............................................10

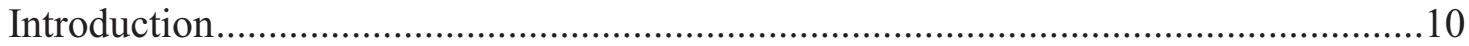

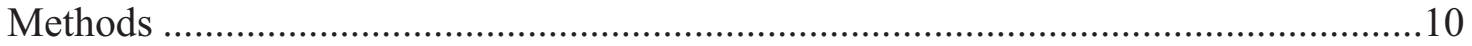

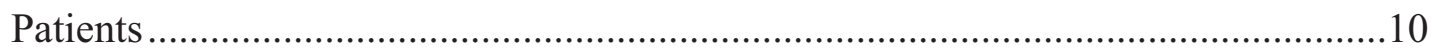

Asparaginase regimen and sample collection .................................................... 10

Phenotyping of clinical allergy to asparaginase .................................................. 12

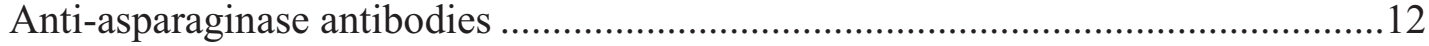

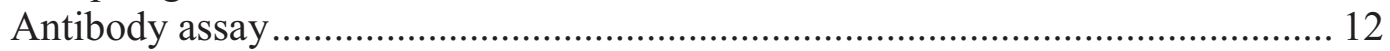

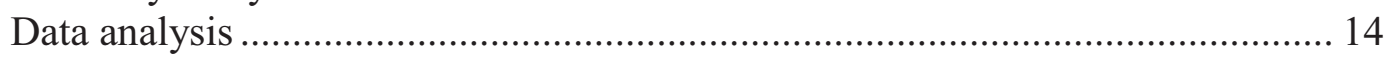

Estimation of antibody area-under-the-curve (AUC) ...................................... 14

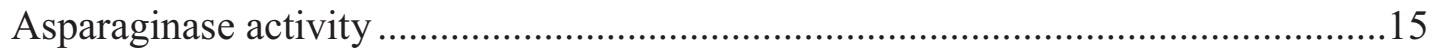

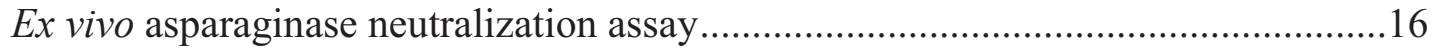

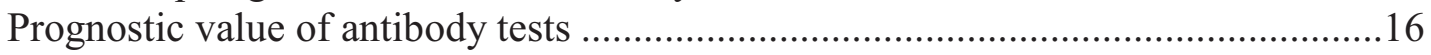

Sensitivity, specificity and predictive values................................................. 16

Receiver-operator-characteristic (ROC) curves............................................... 16

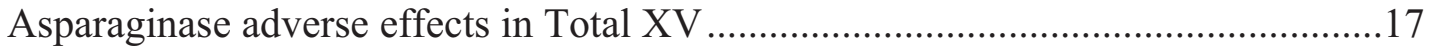

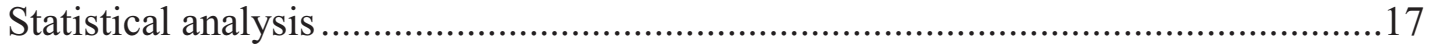

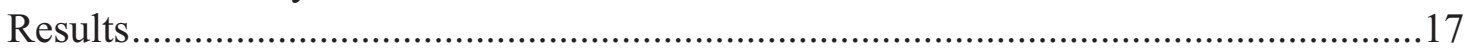

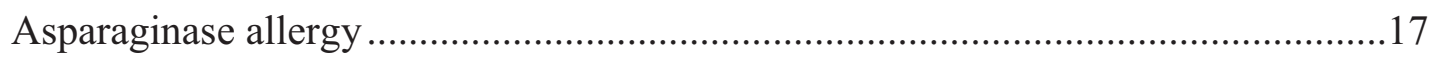

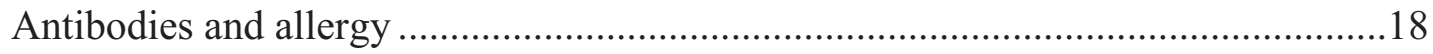

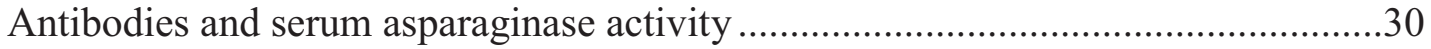

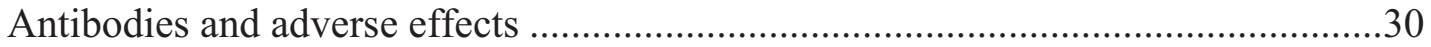

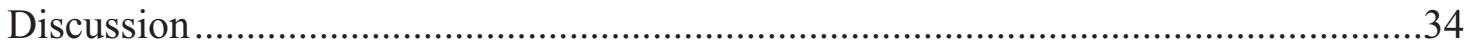

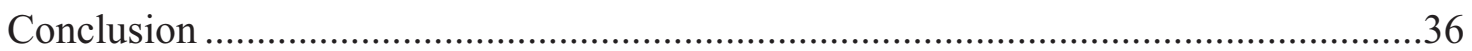




\section{CHAPTER 3. POTENTIATING EFFECT OF ASPARAGINASE ON GLUCOCORTICOID-INDUCED OSTEONECROSIS ..................................................38}

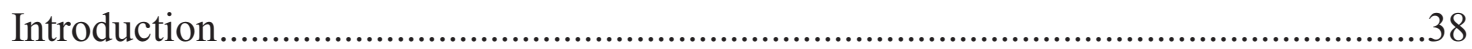

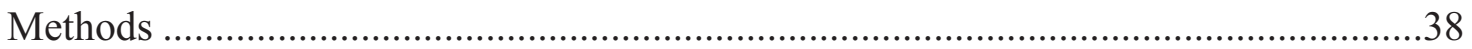

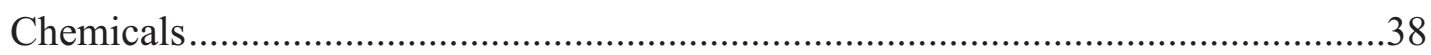

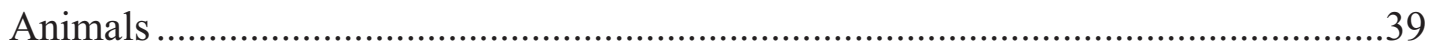

Asparaginase and dexamethasone pharmacokinetics ..................................................39

Mouse model of dexamethasone-induced osteonecrosis .............................................39

Effect of asparaginase on osteonecrosis..................................................................

Plasma dexamethasone concentration and asparaginase activity ................................41

Histological evaluation of osteonecrosis and arteriopathy ........................................41

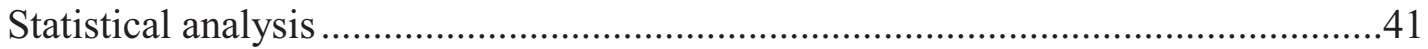

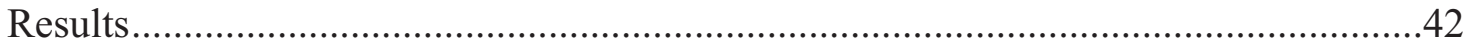

Plasma dexamethasone concentration was increased by asparaginase treatment......42

Asparaginase treatment potentiated osteonecrosis and arteriopathy in

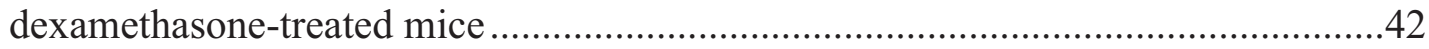

Arteriopathy was likely the initiating event of osteonecrosis ....................................46

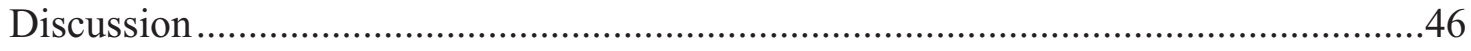

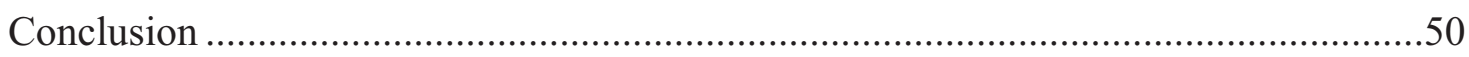

CHAPTER 4. ASPARAGINASE-INDUCED ACUTE PANCREATITIS ..................51

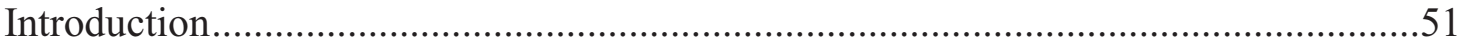

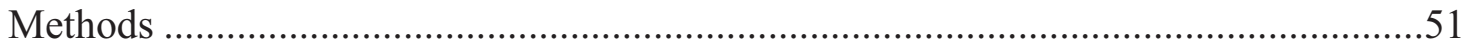

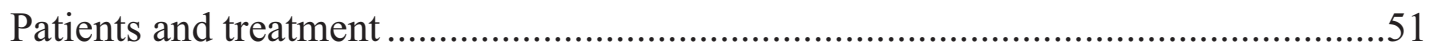

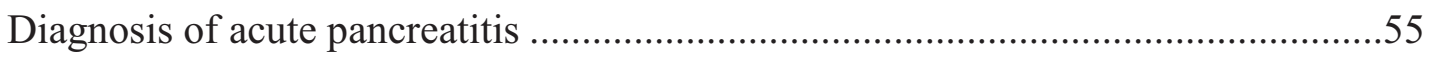

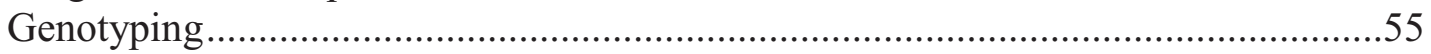

SNP-based analysis for pancreatitis .................................................................58

Gene-level analysis of nonsense variants using SKAT …………...........................58

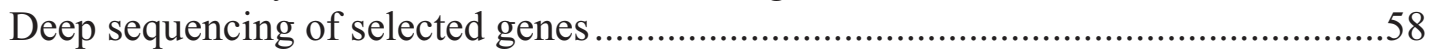

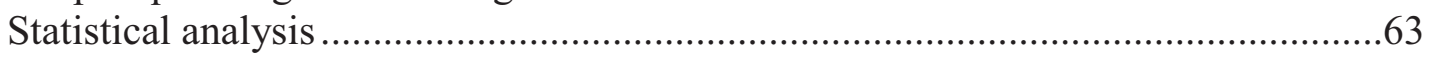

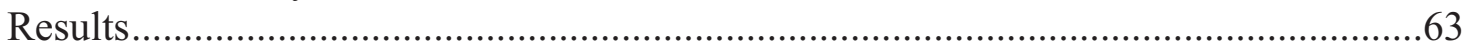

Pancreatitis associated with asparaginase ………………....................................63

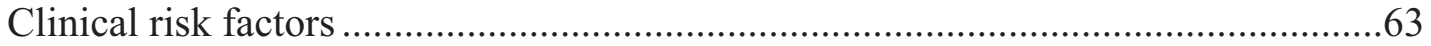

Gene-level analysis of nonsense variants identified $C P A 2 \ldots \ldots \ldots \ldots \ldots \ldots \ldots \ldots \ldots \ldots \ldots \ldots \ldots \ldots . . .68$

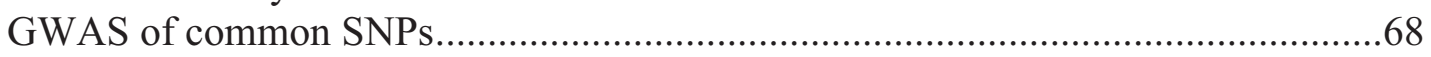

Deep sequencing detected novel variants in CPA2 and other candidate genes ..........77

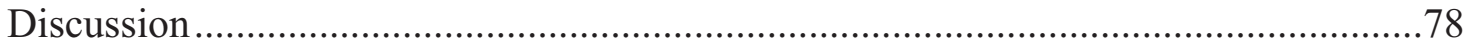

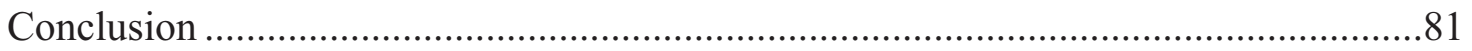

CHAPTER 5. SUMMARY..................................................................................................82

LIST OF REFERENCES ........................................................................................................83

APPENDIX A. SUPPLEMENTARY INFORMATION FOR CHAPTER 2 .............97

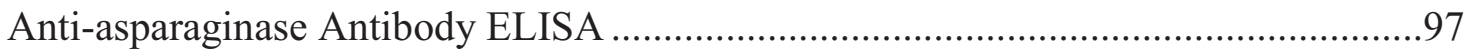

Positive and negative controls ............................................................................97 


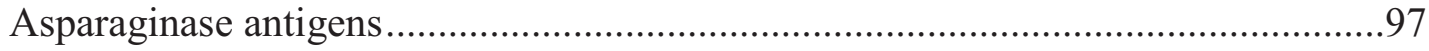

Measurement of anti-asparaginase IgG antibodies by ELISA ..............................97

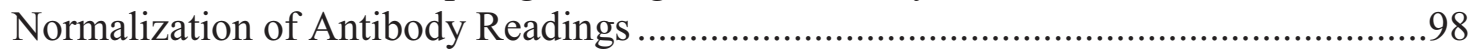

\section{APPENDIX B. SUPPLEMENTARY INFORMATION FOR CHAPTER 3 ..........111}

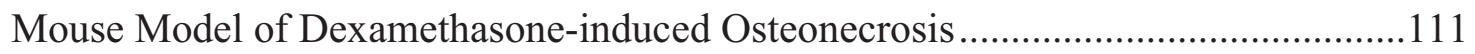

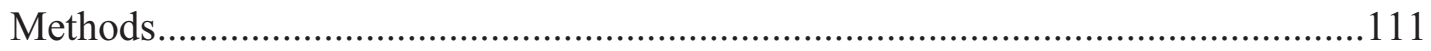

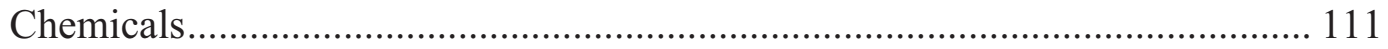

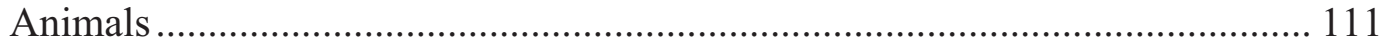

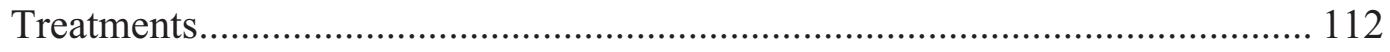

Enteric and blood cultures ...................................................................... 112

Measurement of plasma concentration of dexamethasone and corticosterone... 112

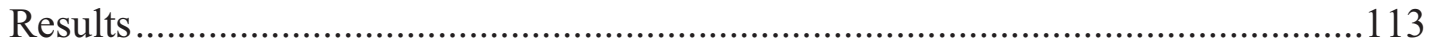

Body weight differed by substrains ............................................................. 113

Effect of substrain, source, age and treatment regimens on survival and development of osteonecrosis ................................................................ 113

Dexamethasone pharmacokinetics and pharmacodynamics ........................... 122

Gender-dependent difference in susceptibility to osteonecrosis ....................... 122

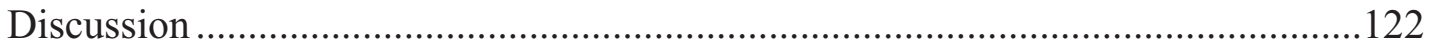

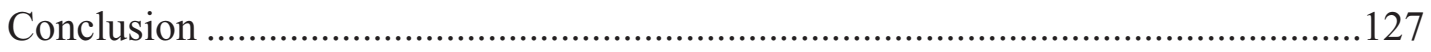

Effect of Asparaginase Treatment on Dexamethasone Pharmacokinetics ..................128

Effect of asparaginase and dexamethasone on plasma protein and lipid levels ..........129

\section{APPENDIX C. SUPPLEMENTARY INFORMATION FOR CHAPTER 4 ..........139}

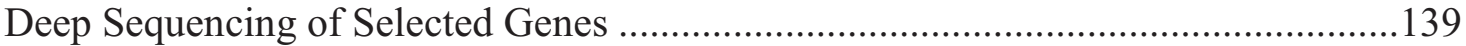

SNP-based and Gene-based Analysis of Variants Identified by Deep Sequencing ....139 


\section{LIST OF TABLES}

Table 2-1. Clinical features of patients with $(n=410)$ and without $(n=88)$ samples evaluable for anti-asparaginase antibodies

Table 2-2. Number of patients with evaluable blood samples at each time point..........13

Table 2-3. Timing of allergic reaction to Elspar ................................................... 19

Table 2-4. Clinical features and allergic reactions to Elspar......................................20

Table 2-5. Clinical features and anti-Elspar antibody status ....................................21

Table 2-6. Sensitivity, specificity and predictive values of three anti-Elspar antibody tests for predicting or confirming clinical reactions to Elspar ......25

Table 2-7. Multivariate analysis showed that low anti-Elspar antibody area-undercurve (AUC) was a risk factor of osteonecrosis $(n=360)$

Table 4-1. Comparison of asparaginase regimens ...............................................52

Table 4-2. Number of patients included in this study ..........................................56

Table 4-3. Putative genes associated with pancreatitis $(n=42)$ selected for deep sequencing based on GWAS result or literature review.

Table 4-4. Multivariate analysis for clinical risk of pancreatitis in the cohort study $(\mathrm{n}=5185)$

Table 4-5. Six genes associated with pancreatitis from the gene-level analysis of nonsense SNPs

Table 4-6. Top 20 common SNPs (cohort risk allele frequency $>1 \%$ ) significantly associated with pancreatitis in the cohort.................................................75

Table A-1. Comparison between antibody OD readings obtained at SJCRH ( $\mathrm{n}=$ $1539)$ and UTHSC $(n=665)$

Table A-2. Sensitivity, specificity and predictive values of anti-Elspar antibody test at week 7 for predicting or confirming clinical reactions to Elspar using current positivity cutoff and optimized cutoff by recursive partitioning ...107

Table B-1. Incidence of $\mathrm{ON}$ in male BALB/c mice from different experiments..... 118

Table B-2. Enteric culture isolates from BALB/cJ mice from vendor and in-house breeding colony 
Table B-3. Inhibition of plasma protein level in human and mouse by asparaginase treatment

Table C-1. Top 10 SNPs from genome-wide analysis of nonsense SNPs; $n=2,357$ SNPS

Table C-2. Top 10 SNPs from genome-wide analysis of missense SNPs (excluding nonsense SNPs); $\mathrm{n}=144,349$ SNPs.

Table C-3. Top 10 SNPs from genome-wide analysis of synonymous and noncoding SNPs; $\mathrm{n}=774,238$ SNPs

Table C-4. All 380 CPA2 variants identified in the cohort $(\mathrm{n}=4217)$ and the casecontrol group $(\mathrm{n}=162)$

Table C-5. Top genes $(P<0.05)$ in the gene-level analysis of nonsense variants identified in putative pancreatitis $(n=42)$ and non-pancreatitis genes $(n$ $=241$ ) based on variants identified by deep sequencing.

Table C-6. SNPs $(\mathrm{n}=81)$ identified by deep sequencing in previously reported pancreatitis-associated candidate genes $(\mathrm{n}=8)$ at the $P<0.05$ level (highlighted) in either the cohort or the case-control group. 


\section{LIST OF FIGURES}

Figure 1-1. Proposed mechanisms of glucocorticoid-induced osteonecrosis ...................

Figure 2-1. Total XV asparaginase regimen and anti-asparaginase antibody

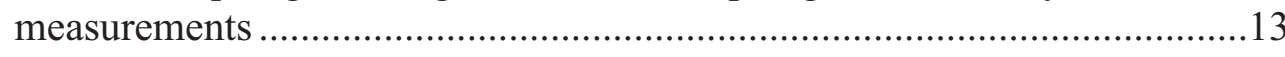

Figure 2-2. Enzyme reactions in asparaginase activity assay .................................. 15

Figure 2-3. Frequency of hypersensitivity to Elspar ................................................ 19

Figure 2-4. Antibody levels in patients with and without clinical reactions .................22

Figure 2-5. Anti-Elspar antibody level relative to the time of clinical reaction to

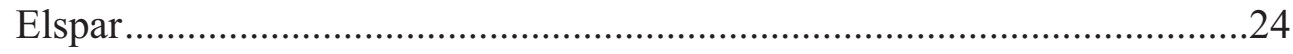

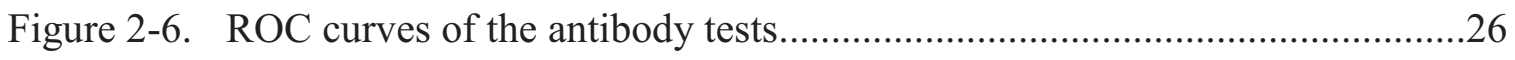

Figure 2-7. Association between week 7 anti-Elspar antibody level and the proportion of patients reacting to Elspar around week 7 .........................28

Figure 2-8. The frequency of hypersensitivity to Oncaspar and Erwinase....................29

Figure 2-9. Correlation between serum asparaginase activity and antibody level .........31

Figure 2-10. Ex vivo neutralization of patient sera with antibodies ................................32

Figure 2-11. Cumulative incidence of osteonecrosis based on anti-asparaginase

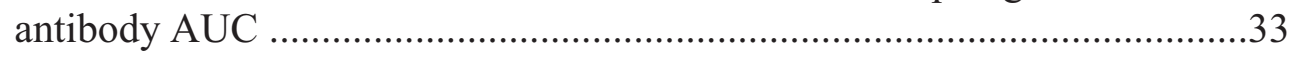

Figure 3-1. Dexamethasone and asparaginase treatment regimens .............................40

Figure 3-2. Plasma dexamethasone concentration was positively associated with asparaginase activity in PK experiment

Figure 3-3. Asparaginase treatment potentiated osteonecrosis and arteriopathy in dexamethasone-treated mice .

Figure 3-4. Osteonecrosis was associated with higher plasma dexamethasone and asparaginase levels in mice receiving both dexamethasone and asparaginase treatment

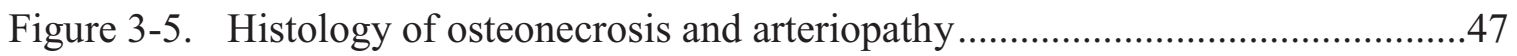

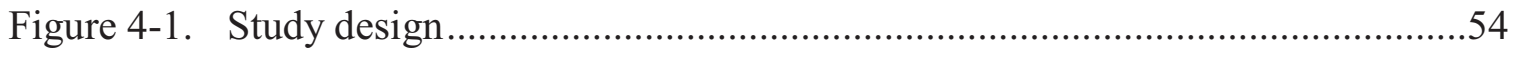

Figure 4-2. Race group assignments based on the ancestral composition of patients in the cohort $(n=5185)$ by treatment protocol 
Figure 4-3. Incidence of pancreatitis differed by protocols

Figure 4-4. Time of pancreatitis in the cohort $(n=5185)$ during the first year of therapy

Figure 4-5. The incidence of pancreatitis based on clinical characteristics .67

Figure 4-6. Association between Native American ancestry and pancreatitis .70

Figure 4-7. CART analysis of clinical risk factors for pancreatitis in the cohort $(\mathrm{n}=$ 5185) .71

Figure A-1. Anti-Elspar OD readings obtained at SJCRH $(\mathrm{n}=1539)$ and UTHSC $(\mathrm{n}$ $=665)$ relative to the time of clinical reaction

Figure A-2. Fitted curves for (A) anti-Elspar, (B) anti-Erwinase and (C) antiOncaspar OD readings of the samples $(n=194)$ measured at both SJCRH and UTHSC.

Figure A-3. UTHSC OD readings before and after normalization................................105

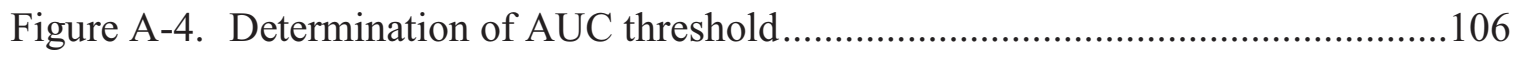

Figure A-5. Week-7 optimal threshold of OD value to predict or to confirm clinical reactions

Figure A-6. Elspar has stronger cross-reactivity with Oncaspar than with Erwinase ....109

Figure A-7. Sensitivity of antibody tests within 30 days of reactions......

Figure B-1. Body weight of mice before and after treatment

Figure B-2. Incidence of osteonecrosis increased with longer treatment duration

Figure B-3. Similar histologic appearance of osteonecrosis in mice of different substrain, age and treatment groups

Figure B-4. Survival differed by substrains, sources, regimens and age of mice 119

Figure B-5. Plasma concentrations of dexamethasone 123

Figure B-6. Inverse association between plasma corticosterone and dexamethasone levels.

Figure B-7. Gender-dependent differences in susceptibility to osteonecrosis .....

Figure B-8. Plasma dexamethasone concentration was positively associated with asparaginase activity in osteonecrosis experiment. 
Figure B-9. Mouse plasma amylase level after treatment with dexamethasone and asparaginase

Figure B-10. Mouse plasma lipase level after treatment with dexamethasone and asparaginase

Figure B-11. Mouse plasma triglyceride level after treatment with dexamethasone and asparaginase

Figure B-12. Mouse plasma albumin level after treatment with dexamethasone and asparaginase

Figure B-13. Mouse plasma cortisol level after treatment with dexamethasone and asparaginase

Figure B-14. Correlation between asparagine content in the protein and the extent of inhibition by asparaginase treatment

Figure C-1. CADD score in all SNPs on exome array $(\mathrm{n}=162586)$ and pancreatitisassociated SNPs $(P<0.05 ; \mathrm{n}=8018)$

Figure C-2. Top pathways and functions of pancreatitis-associated genes $(n=42) \ldots \ldots 163$

Figure C-3. Top pathways and functions of pancreatitis-associated genes identified by GWAS $(\mathrm{n}=34)$ 


\section{LIST OF ABBREVIATIONS}

ABL

ACTN2

ALL

ANTI-ASP

AP

ASNS

ASP

AUC

BCR

CADD

CART

CASR

CFTR

CI

CLDN2

CNS

COG

CPA1

CPA2

CPB

$\mathrm{CSF}$

CTRC

CYP

DEX

DI

DOCK5

ELISA

ER

ExAC

FDA

FHIT

FN

FP

GEF

GWAS

HLA

HOGA1

HPLC

HR

IACUC

Ig

IM

I.m.
Abelson tyrosine protein kinase

Actinin-2

Acute lymphoblastic leukemia

Anti-asparaginase antibodies

Acute pancreatitis

Asparagine synthetase

Asparaginase

Area-under-the-curve

Breakpoint cluster region

Combined Annotation Dependent Depletion

Classification and regression tree

Calcium sensing receptor

Cystic fibrosis trans-membrane conductance regulator

Confidence interval

Claudin-2

Central nervous system

Children's Oncology Group

Carboxypeptidase A1

Carboxypeptidase A2

Carboxypeptidase B

Cerebrospinal fluid

Chymotrypsionogen $\mathrm{C}$

Cytochrome P450

Dexamethasone

Delayed intensification

Dedicator of cytokinesis 5

Enzyme-linked immunosorbent assay

Endoplasmic reticulum

Exome Aggregation Consortium

U.S. Food and Drug Administration

Fragile histidine traid protein

False negative

False positive

Guanine nucleotide exchange factor

Genome-wide association study

Human leukocyte antigen

4-hydroxy-2-oxoglutarate aldolase

High-performance liquid chromatography

Hazard ratio

Institutional Animal Care and Use Committee

Immunoglobulin

Interim maintenance

Intramuscularly 


\begin{tabular}{|c|c|}
\hline I.p. & Intraperitoneally \\
\hline IU & International unit(s) \\
\hline I.v. & Intravenously \\
\hline LOQ & Limit of quantitation \\
\hline LR & Low-risk \\
\hline MAF & Minor allele frequency \\
\hline MICAL2 & Molecule interacting with CasL protein 2 \\
\hline MRD & Minimal residual disease \\
\hline MRI & Magnetic resonance imaging \\
\hline MS & Mass spectrometric \\
\hline MSC & Mesenchymal stem cells \\
\hline NADH & Nicotinamide adenine dinucleotide (reduced) \\
\hline NCI CTCAE & $\begin{array}{l}\text { National Cancer Institute Common Toxicity Criteria for Adverse } \\
\text { Events }\end{array}$ \\
\hline NHP & Normal human plasma \\
\hline NPV & Negative predictive value \\
\hline OD & Optical density \\
\hline $\mathrm{ON}$ & Osteonecrosis \\
\hline OR & Odds ratio \\
\hline P24 (or P28) & Postnatal day 24 (or 28 ) \\
\hline PBS & Phosphate-buffered saline \\
\hline PEG & Polyethylene glycol \\
\hline PK & Pharmacokinetics \\
\hline PPV & Positive predictive value \\
\hline PRSS1 & Protease serine 1 (cationic trypsinogen) \\
\hline PRSS2 & Protease serine 2 (anionic trypsinogen) \\
\hline PSTI & Pancreatic secretory trypsin inhibitor \\
\hline RAF & Risk allele frequency \\
\hline ROC & Receiver-operator-characteristic \\
\hline RSID & Reference SNP identification number \\
\hline SD & Standard deviation \\
\hline SE & Standard error \\
\hline SHR & Standard/high-risk \\
\hline SJCRH & St. Jude Children's Research Hospital \\
\hline SKAT & Sequence kernel association test \\
\hline SMZ & Sulfamethoxazole \\
\hline SNP & Single nucleotide polymorphism \\
\hline SPINK1 & Serine protease inhibitor kazal type 1 \\
\hline TCN & Tetracycline \\
\hline TMP & Trimethoprim \\
\hline $\mathrm{TN}$ & True negative \\
\hline $\mathrm{TP}$ & True positive \\
\hline ТРMT & Thiopurine S-methyltransferase \\
\hline UTHSC & University of Tennessee Health Science Center \\
\hline UTR & Untranslated region \\
\hline U & Ultraviolet \\
\hline
\end{tabular}




\section{CHAPTER 1. BACKGROUND}

\section{Acute Lymphoblastic Leukemia (ALL)}

ALL is a malignant disorder of lymphoid progenitor cells, characterized by proliferation of immature white blood cells - known as lymphoblasts. The lymphoblasts invade peripheral blood, bone marrow and other organs, causing tissue damage and inhibition of normal cells. The symptoms of ALL include weakness, fever, pain in bone and joints, bruising and bleeding, petechiae and others. Diagnostic methods include blood tests, bone marrow aspiration and biopsy, lumbar puncture and chest X-ray. ${ }^{1}$ The immunophenotype of lymphoblasts (B- or T-lineage) can be identified by their surface antigens using flow cytometry. ${ }^{2}$

ALL is the most common childhood cancer and accounts for $25 \%$ of cancer diagnoses in children younger than 15 years, with peak prevalence between 2 and 5 years. ${ }^{3}$ The incidence of new diagnosis every year is approximately 4 cases per million for children and adolescents under 20 years, with an upward trend over the past 40 years. ${ }^{4}$ The increasing incidence may be associated with changes in environmental factors, such as industrialization. In addition, the improved diagnosis and access to medical care over time may also play a role. ${ }^{4}$

Although the precise pathogenesis of ALL remains unknown, this disease has been associated with genetic alterations in tumor-suppressors, proto-oncogenes and other key regulatory genes of hematopoietic stem cells and progenitors. ${ }^{1,5}$ In about $75 \%$ of childhood ALL cases, recurring chromosomal alterations have been detected. ${ }^{6}$ For example, the $\mathrm{t}(9 ; 22)$ chromosomal translocation results in fusion of the breakpoint cluster region $(B C R)$ on chromosome 22 with the Abelson tyrosine protein kinase $1(A B L 1)$ on chromosome 9 , which is a tyrosine kinase whose activity is well regulated under normal conditions. The fused BCR-ABL protein, however, has high constitutive activity that leads to enhanced proliferation and self-renewal of hematopoietic stem cells. ${ }^{7}$

There has been dramatic increase in the survival rate of children and adolescents with ALL. In the past few decades, the five-year event-free survival rate of pediatric patients has been improved from less than $5 \%$ to over $80 \%$, and the overall survival rate has reached $90 \%{ }^{5,8}$ Success was achieved by the intense use of chemotherapeutic drugs, such as asparaginase, glucocorticoids and thiopurines. Meanwhile, with such a high cure rate, investigators and clinicians have also turned their attention to improving the quality of life of ALL survivors. In recent years, many efforts have been made to modify ALL therapies in attempts to achieve optimal efficacy and avoid serious toxicities, which requires a clear understanding of the mechanisms of drug response and the risk factors of adverse drug reactions. 


\section{Asparaginase}

\section{Introduction}

In $1953, \mathrm{Kidd}^{9,10}$ first observed that injections of normal guinea pig serum induced regression of transplanted lymphoma in mice and rats. At that time it was believed to be an immunological phenomenon. In 1963, Broome ${ }^{11}$ found out that an enzyme, asparaginase, was responsible for the anti-tumor activity. Mashburn and Wriston $^{12}$ subsequently showed that L-asparaginase derived from Escherichia coli had similar tumor inhibitory effect. The first clinical use of asparaginase, which was purified from guinea pig serum, showed a short therapeutic effect in a pediatric patient with relapsed ALL. ${ }^{13}$ Later the development of isolation and purification procedures of Lasparaginase from E.coli has enabled large-scale production of this effective chemotherapeutic agent. $^{14}$

Asparaginase is an enzyme consisting of four identical subunits and has a molecular weight of $140000 \mathrm{Da}$. It catalyzes the hydrolysis of a non-essential amino acid, asparagine, to aspartic acid and ammonia. Unlike other tissues, leukemic lymphoblasts are deficient of asparagine synthetase (ASNS) and therefore are sensitive to depletion of circulating asparagine. It has been reported that asparaginase sensitivity was correlated with ASNS protein content in ALL cell lines. ${ }^{15}$ Moreover, bone marrow-derived mesenchymal stem cells (MSCs), which form the microenvironment for leukemic cells to grow, have been shown to have high expression of ASNS. ${ }^{16}$

Asparaginase also shows glutaminase activity, which catalyzes the hydrolysis of glutamine to glutamate. For E.coli- and Erwinia chrysanthemi-derived asparaginase, glutaminase activity is about $5 \%$ of asparaginase activity. ${ }^{17}$

\section{Formulations}

There are currently three formulations of asparaginase available for the treatment of patients with ALL: the native E.coli asparaginase, a pegylated (polyethylene glycolconjugated) form of native E.coli asparaginase (PEG-asparaginase), and Erwinia chrysanthemi-derived asparaginase.

\section{Native E.coli asparaginase}

In 1978, Native E.coli asparaginase was approved by the U.S. Food and Drug Administration (FDA) as a component of a multi-agent chemotherapeutic regimen for the treatment of patients with ALL. It has been the most widely used asparaginase formulation, and common brand names include Elspar (USA), Kidrolase (UK), Leunase (France), Crasnitin (Germany) and Asparaginase Medac (Japan). Some of the brand 
names have been discontinued, and Native E.coli asparaginase is no longer available as a pharmaceutical preparation in the U.S.

The half-life of native E.coli asparaginase is approximately 1.2 days. ${ }^{18}$ Elspar is stored as lyophilized powder, and is typically given intramuscularly (i.m.) at 6000-10000 $\mathrm{U} / \mathrm{m}^{2}$ three times a week. Although asparaginase cannot cross the blood-brain barrier, it can deplete asparagine in the cerebrospinal fluid (CSF). ${ }^{19}$ Serum level of asparaginase at 100 international units (IU)/L was considered adequate to fully deplete asparagine in blood and CSF. ${ }^{20}$ It has been reported that the presence of neutralizing antibodies against native E.coli asparaginase was associated with higher risk of relapse in the central nervous system (CNS). ${ }^{21}$

\section{PEG-asparaginase}

PEG-conjugation has substantially prolonged the circulating half-life and decreased the immunogenicity of native E.coli asparaginase. ${ }^{22}$ PEG-asparaginase (or pegaspargase, brand name: Oncaspar) was first used as a second-line option for patients who developed hypersensitivity to native E.coli asparaginase. It was approved for the first-line use by FDA in $2006 .^{23}$ PEG-asparaginase has replaced native E.coli asparaginase in some recent ALL trials ${ }^{24}$ because it has a longer half-life (about 5.7 days), ${ }^{18}$ thus requires a reduced dose and frequency $\left(2500-3500 \mathrm{U} / \mathrm{m}^{2}\right.$ every two weeks) comparing to native E.coli asparaginase. The disadvantages of PEG-asparaginase include its high cost and short shelf-life (about 6-8 months).

\section{Erwinia asparaginase}

Erwinia asparaginase (brand name: Erwinaze in USA or Erwinase in UK) was approved by FDA as second-line therapy when patients develop allergy to native E.coli asparaginase or PEG-asparaginase for ALL in 2011. ${ }^{25}$ Erwinia asparaginase has a short half-life of 0.6 day. ${ }^{18}$ It has been shown to have lower serum activity and therapeutic efficacy than native E.coli asparaginase. ${ }^{26}$ In EORTC-CLG 58881 trial, patients receiving Erwinia asparaginase had inferior outcome comparing to those receiving native E.coli asparaginase at the same dose $\left(10000 \mathrm{U} / \mathrm{m}^{2}\right.$ twice weekly). ${ }^{27}$ Therefore, higher dose (20000-30000 U/m ${ }^{2}$ ) and more frequent administration (three times a week) is recommended when using Erwinia asparaginase. Recently, a pegylated recombinant Erwinia asparaginase (PEGcrisantaspase) has been reported to have similar efficacy and lower immunogenicity than native Erwinia asparaginase in a preclinical study. ${ }^{28}$

\section{Adverse effects}

The use of asparaginase is complicated by its adverse effects. Common adverse effects include hypersensitivity, thrombosis, coagulopathy, pancreatitis, altered lipid metabolism, glucose intolerance, hepatotoxicity and neurotoxicity. ${ }^{29}$ Drug interactions 
between asparaginase and other antileukemic agents, such as glucocorticoids, have also been observed. ${ }^{30}$

\section{Hypersensitivity}

Because asparaginase is a bacteria-derived protein, hypersensitivity reaction is the most frequent adverse effect. Up to $45 \%$ patients can develop clinical allergy to native $E$. coli asparaginase, mostly upon re-exposure. ${ }^{31}$ Some patients may also develop antiasparaginase antibodies without any clinical signs of hypersensitivity, which is referred to as "silent hypersensitivity". 32 Anti-asparaginase antibodies can be detected by enzymelinked immunosorbent assay (ELISA) in patients with hypersensitivity to asparaginase. The majority of anti-asparaginase antibodies are of the immunoglobulin $\mathrm{G}$ ( $\mathrm{IgG}$ ) class, and the presence of IgM and IgE has been reported in several cases. ${ }^{33-36}$

Allergic reactions typically require discontinuation of the offending formulation (e.g., native E. coli asparaginase) and substitution with other formulations (e.g., Erwinia asparaginase or PEG-asparaginase). Only $20-25 \%$ of patients will develop allergic reactions to the substituting formulation. ${ }^{18}$ However, allergic reactions caused by asparaginase should be differentiated from acute reactions caused by other drugs. So far only few studies have focused on using a diagnostic test, such as anti-asparaginase antibodies, to confirm allergic reactions. ${ }^{37}$

Attempts have been made to predict asparaginase hypersensitivity, especially the life-threatening anaphylactic reactions, before asparaginase is given. Some studies used a small test dose to determine the reactivity of patients; however, this method seemed to lack sensitivity and the test dose itself may pre-sensitize the patients. ${ }^{38,39}$

Anti-asparaginase IgG antibodies were associated with lower serum asparaginase activity and a decreased half-life of asparaginase. ${ }^{32,40,41}$ Moreover, because of the crossreactivity between native and pegylated E.coli asparaginase, the activity of PEGasparaginase as a second-line treatment was also reported to be associated with antiE.coli asparaginase antibody levels. ${ }^{42}$ The presence of $\mathrm{IgG}$ antibodies has been shown to affect the therapeutic outcome of ALL, ${ }^{21,43}$ although there are conflicting data. ${ }^{31,36,44-46}$ There was no correlation between serum asparaginase activity and IgM or IgE antibody levels. 33

Thus, we studied the effect of anti-asparaginase (Elspar) IgG antibodies on serum asparaginase activity, and investigated the clinical utility of anti-Elspar antibodies (see Chapter 2).

\section{Acute pancreatitis}

Acute pancreatitis is one of the most serious adverse events of asparaginase treatment. It occurs in up to $18 \%$ of patients, ${ }^{40,47-54}$ and can be life-threatening for some 
patients who develop pseudocysts or necrosis. ${ }^{55,56}$ Common symptoms associated with pancreatitis include nausea, vomiting, and upper abdominal pain especially after eating. ${ }^{57}$ The diagnostic criteria include (a) upper abnominal pain, (b) serum level of amylase or lipase $\geq 3$ times the upper limit of normal, and (c) radiographical findings consistent with pancreatitis. The grading system is based on the National Cancer Institute Common Toxicity Criteria for Adverse Events (NCI CTCAE) ${ }^{58}$ or the Atlanta Classification system. $^{59}$

Development of pancreatitis during asparaginase treatment can result in temporarily or permanently withholding of asparaginase based on the severity of the initial episode. Some of the patients may be allowed to receive asparaginase after recovery if their initial episode is considered mild. However, up to $63 \%$ of patients may experience a second episode of pancreatitis after being re-challenged. ${ }^{60}$ There is no definitive treatment for pancreatitis; only supportive care is given. ${ }^{57,61}$

The pathophysiology behind asparaginase-induced pancreatitis remains unknown. It is hypothesized that asparaginase treatment may be associated with amino acid imbalance and altered pancreatic secretion. After pediatric patients with ALL received the first dose of asparaginase, an imbalance of plasma amino acid levels was observed along with elevations of trypsin and pancreatic secretory trypsin inhibitor (PSTI), a sign of pancreatic injury. ${ }^{62}$ In a rat model of asparaginase-induced "pre-pancreatitis", which was characterized by slight morphologic change without enzyme elevation, it was observed that zymogen granules accumulated within pancreatic acinar cells, indicating that asparaginase may alter exocrine function of pancreas. ${ }^{63}$ Asparaginase has also been shown to affect insulin response, which can be attributed to an impairment of exocrine secretion. ${ }^{64}$ However, the underlying mechanism has not been elucidated.

Older age and intensive asparaginase therapy have been indicated to increase the risk of pancreatitis in pediatric patients with ALL, ${ }^{47-50,56,65}$ although most studies of asparaginase-induced pancreatitis lack sufficient power to robustly assess risk factors. ${ }^{46-49}$ Genetic predispositions may also play a role, because some patients develop pancreatitis after only few doses of asparaginase. Several genetic factors have been identified for other forms of pancreatitis, mostly associated with premature trypsin activation or sustained trypsin activity in the pancreas. All of these studies focused on pancreatitis of other etiologies, such as alcoholic, idiopathic and chronic pancreatitis. There has been no genetic study on asparaginase-induced pancreatitis. ${ }^{82}$ Gain-of-function mutations in cationic and anionic trypsinogen genes (PRSS1 and PRSS2) have been associated with pancreatitis. ${ }^{66-69}$ Polymorphisms in serine protease inhibitor kazal type 1 (SPINK1), a gene encoding an inhibitor that prevents premature trypsin activation, have been shown to be associated with pancreatitis. ${ }^{70-72}$ Cystic fibrosis trans-membrane conductance regulator (CFTR), which regulates elimination of trypsin through the pancreatic duct, has also been linked to pancreatitis. ${ }^{73-75}$ Other risk genes include chymotrypsinogen $\mathrm{C}$ $(C T R C)$, calcium sensing receptor (CASR), claudin-2 (CLDN2), carboxypeptidase 1 (CPA1), and HLA-DRB1*07:01. ${ }^{72,75-81}$ 
In our current study, we aimed to identify the clinical and genetic risk factors for asparaginase-induced acute pancreatitis in pediatric patients with ALL (see Chapter 4).

\section{Drug interaction with glucocorticoids}

Because asparaginase inhibits the synthesis of protein, including enzymes responsible for drug metabolism and DNA synthesis, it may have interactions with accompanying antileukemic medication, such as glucocorticoids.

Glucocorticoids (e.g. dexamethasone and prednisone) are a class of steroid hormones with anti-inflammatory activities, thus are used extensively in adult and pediatric oncology. ${ }^{83-86}$ Asparaginase exposure has been shown to be associated with decreased clearance and increased exposure of dexamethasone. ${ }^{30}$ Moreover, a clinical trial reported that the development of an antibody response against asparaginase was associated with a decreased incidence of glucocorticoid-induced osteonecrosis, ${ }^{87}$ a common adverse effect of glucocorticoids. ${ }^{88-92}$

\section{Effect on glucocorticoid-induced osteonecrosis}

Osteonecrosis is one of the primary dose-limiting toxicities of ALL therapy. ${ }^{88-92}$ The development of osteonecrosis can result in debilitation and surgical intervention, and seriously affect the quality of life. Proposed mechanisms (Figure 1-1) of glucocorticoidinduced osteonecrosis include inhibition of angiogenesis, bone marrow adipogenesis, hypercoagulation, and apoptosis of endothelial cells and osteocytes. ${ }^{93}$ Our study recently suggested that damage to blood vessels supplying bone (refered to as arteriopathy) may play a primary role in the pathogenesis of osteonecrosis. ${ }^{94}$

Evidence is accumulating that asparaginase can increase the risk of glucocorticoid-induced osteonecrosis. A recent ALL trial showed that additional doses of asparaginase during interim maintenance therapy caused more osteonecrosis in patients receiving prednisone. ${ }^{95}$ The hypoalbuminemia caused by asparaginase was also associated with osteonecrosis risk. ${ }^{90}$ However, these clinical data are not definitive, in that they do not come from a trial in which patients receive identical glucocorticoid regimens with different exposures to asparaginase.

Because any modification of current chemotherapy to decrease the risk of adverse events must be weighed against the possible compromised antileukemic effect, preclinical models are needed to characterize the contribution of concurrent medications, different dosing schedules and genetic variations to glucocorticoid-induced osteonecrosis, and will be coupled with studies to evaluate the influence of these risk factors for antileukemic effectiveness. Our lab has developed the first murine model for glucocorticoid-induced osteonecrosis. ${ }^{96}$ Male BALB/cJ mice were treated with oral dexamethasone continuously for 12 weeks, and the incidence of osteonecrosis was approximately $45 \%$. In past few years we have further optimized the murine model, 


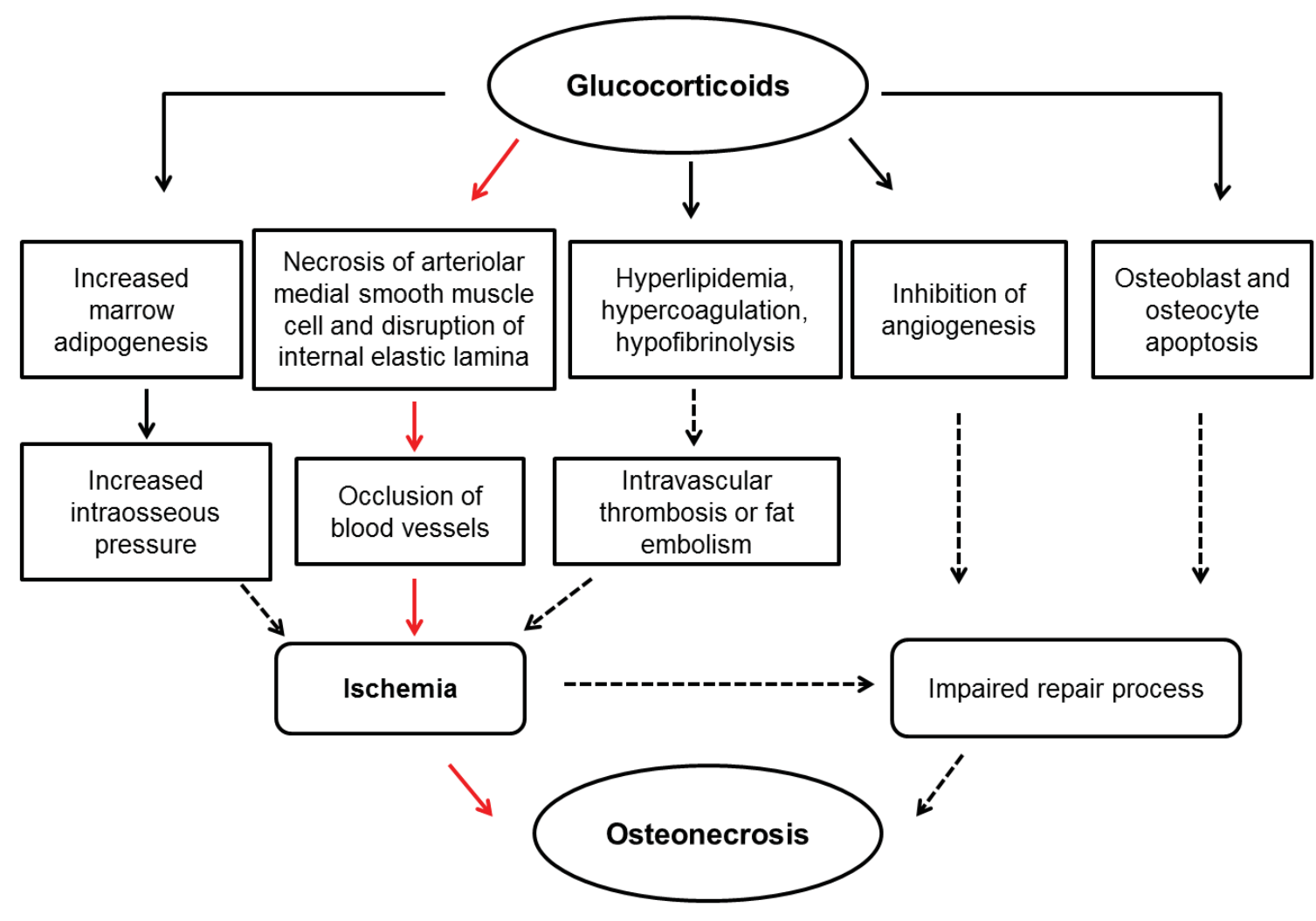

Figure 1-1. Proposed mechanisms of glucocorticoid-induced osteonecrosis

Solid arrows represent the relationships that have been experimentally confirmed, and dashed arrows represent hypothesized relationships. Red arrows show the mechanism that involves damage to blood vessels supplying bone (arteriopathy). ${ }^{94}$ 
and here we utilized this model to study the effect of asparaginase on glucocorticoidinduced osteonecrosis (see Chapter 3).

\section{Pharmacogenomics: A Brief Introduction}

Pharmacogenomics is the study of the role of genetics in drug response. There is substantial interpatient variability in response to many medications, including drug absorption, distribution, metabolism and excretion ("pharmacokinetics"), and the pharmacologic effects of drugs on the body ("pharmacodynamics"). These individual differences can be caused by genetic polymorphisms (e.g. single-nucleotide polymorphism, SNP) in genes encoding drug-metabolizing enzymes, drug transporters or drug targets or modifiers. Given the narrow therapeutic index of anticancer drugs, especially for pediatric patients, it is particularly important to optimize the treatment for both safety and efficacy. ${ }^{97}$ Moreover, pharmacogenomic studies also yielded new insights into the pathogenesis of disease and underlying mechanisms of drug response. ${ }^{66,69,80,90,98}$ The ultimate goal of pharmacogenomics is to provide personalized treatment with maximal efficacy and minimal toxicity for individual patients - also known as "personalized medicine".

Over the past half century, advances of genomic technology have enabled a more concise, comprehensive and systematic view of the genome. Currently, the major challenge is to use appropriate study designs and analysis strategies to elucidate the complex genotype-phenotype relationships. Two primary types of designs are cohort and case-control studies, and two common strategies are genome-wide association study (GWAS) and the candidate-gene approach. ${ }^{98}$

\section{Cohort study}

A cohort means a group of people who share a common characteristic (e.g. pediatric patients treated for ALL). Cohort studies assess the outcome status of each individual in the cohort, thus limit the potential of biases and provide strong generalizability power. Cohort studies can be prospective (from the present time to the future) or retrospective (from the present time to the past). Prospective studies have welldesigned data collection methods that allow the investigators to collect complete and specific data, but it may be less efficient when studying diseases with long follow-up period. In contrast, retrospective studies are more cost- and time-effective, but the investigators have very limited control over data collection, ${ }^{99}$ including data on factors needed for correcting bias.

\section{Case-control study}

Case-control studies identify subjects by their outcome status, therefore are retrospective studies. Subjects are categorized as "cases" and "controls", and the 
association between outcomes and exposures are determined. Comparing with cohort studies, case-control studies requires fewer subjects; however, their biggest disadvantage is their susceptibility to biases. The selection of matching controls is thus critical - each control subject should be paired with a case with respect to the background variables in order to ensure comparability. ${ }^{100}$ However, because important covariates are not always known, controls may not be appropriately matched to cases, which could lead to bias. In addition, as the interpretation largely hinges on the definitions of "cases" and "controls", great caution must be taken when generalizing to study population.

\section{Candidate-gene approach}

The candidate-gene approach has been the traditional strategy of pharmacogenomics studies, especially before the development of large-scale and highthroughput genotyping techniques. The selection of single-gene candidates is based on our knowledge about the medication, and most of the candidates are key metabolizing enzymes. One of the well-known successes was thiopurine S-methyltransferase (TPMT), an enzyme responsible for inactivation of thiopurine drugs. Polymorphisms in TPMT have been found to be associated with increased levels of active thiopurine metabolites and bone marrow suppression in patients receiving thiopurines. ${ }^{101}$ It has been recommended that TPMT variants should be tested before starting treatment with thiopurines. ${ }^{102}$ The disadvantage of candidate gene approach is that it requires a priori knowledge, which may limit the ability to include all possible causative genes and polymorphisms. ${ }^{103}$

\section{GWAS}

In contrast to candidate-gene approach, GWAS involves an scan of the genome, interrogating all genetic variations for phenotype association. The advantage of GWAS is clear - it can identify totally novel genes and lead to important insights into the overall mechanisms of drug response. With high sample sizes, GWAS has adequate power to allow detection of common genetic variations with modest to high effect sizes, ${ }^{104}$ but the ability of testing rare variation by GWAS approach can be limited by genotyping platforms, because most DNA microarrays have been designed to capture common variations. ${ }^{105}$ Deep re-sequencing of the regions implicated by GWAS may be effective in revealing the functional rare variants. ${ }^{106}$

There are currently fewer genome-wide studies on adverse effects of drugs than there are on drug response, probably because of the limited sample sizes. ${ }^{107}$ However, prediction of the potential toxicity, especially the serious and rare adverse events, may provide important guidance for personalized medication. Understanding the genetic factors that predispose patients to adverse effects can help us discover the underlying mechanism, identify patients at risk, and develop clinical tests and treatment regimens to reduce the toxicity. ${ }^{108}$ In Chapter 4 , we will present a comprehensive study of genetic risk factors associated with asparaginase-induced pancreatitis in pediatric patients with ALL. 


\section{CHAPTER 2. ASPARAGINASE HYPERSENSITIVITY*}

\section{Introduction}

Asparaginase is a critical treatment component for ALL; ${ }^{109-113}$ but its use is complicated by the development of clinical hypersensitivity. ${ }^{33,37,114-117}$ Differentiating allergy to asparaginase from other acute reactions can sometimes be challenging. Serum asparaginase antibody has been associated with clinical allergy, although few studies have focused on its utility as a diagnostic test. ${ }^{37}$

Some previous studies have indicated that serum antibodies, even in the absence of clinical allergy, may inhibit serum asparaginase activity ${ }^{40}$ and attenuate its anticancer effect, ${ }^{43}$ although there are conflicting data. ${ }^{31,36,44-46}$ However, many studies lack properly timed control samples from patients whose asparaginase therapy is identical to that of patients who do develop antibodies.

Here, we prospectively measured IgG antibodies to asparaginase at predetermined time points among 410 pediatric patients treated on a front-line trial of ALL, St. Jude Total XV protocol, and evaluated the predictive utility of antibody measures for allergy, and their association with asparaginase activity and adverse effects.

\section{Methods}

\section{Patients}

Between 2000 and 2007, 498 patients with newly diagnosed childhood ALL were enrolled in St. Jude Children's Research Hospital (SJCRH) front-line Total XV protocol: 239 treated on the low-risk (LR) arm and 259 on the standard/high-risk (SHR) arm. All patients received asparaginase treatment, and 410 (197 LR and 213 SHR) had serum samples evaluable for anti-asparaginase antibodies (Table 2-1). The risk arm assignment and detailed treatment regimens have been described previously. ${ }^{8}$ Genetic ancestry was estimated using STRUCTURE ${ }^{118}$ and race was categorized as white (European ancestry > 90\%), black (African ancestry $>70 \%$ ) and other.

\section{Asparaginase regimen and sample collection}

During remission induction, Elspar was administered i.m. at a dose of 10000 $\mathrm{U} / \mathrm{m}^{2}$ thrice weekly, for a total of 6 doses (on days $6,8,10,12,14$, and 16) or 9 doses

*Adapted with permission. Liu C, Kawedia JD, Cheng C, et al. Clinical utility and implications of asparaginase antibodies in acute lymphoblastic leukemia. Leukemia. Nov 2012;26(11):2303-2309. 
Table 2-1. Clinical features of patients with $(n=410)$ and without $(n=88)$ samples evaluable for anti-asparaginase antibodies

\begin{tabular}{|c|c|c|c|}
\hline Clinical features & $\begin{array}{c}\text { Patients with samples } \\
\text { n (\%) }\end{array}$ & $\begin{array}{c}\text { Patients without samples } \\
\text { n (\%) }\end{array}$ & $P$ \\
\hline \multicolumn{4}{|l|}{ Age } \\
\hline$<10$ years & $302(81.2)$ & $70(18.8)$ & 0.28 \\
\hline$\geq 10$ years & $108(85.7)$ & $18(14.3)$ & \\
\hline \multicolumn{4}{|l|}{ Sex } \\
\hline Male & $228(81.7)$ & $51(18.3)$ & 0.72 \\
\hline Female & $182(83.1)$ & $37(16.9)$ & \\
\hline \multicolumn{4}{|l|}{$\operatorname{Race}^{\mathrm{a}}$} \\
\hline White & $282(88.4)$ & $37(11.6)$ & 0.0001 \\
\hline Black & $65(91.5)$ & $6(8.5)$ & \\
\hline Other & $63(58.3)$ & $45(41.7)$ & \\
\hline \multicolumn{4}{|l|}{ Immunophenotype $\mathrm{e}^{\mathrm{b}}$} \\
\hline B-lineage & $345(81.6)$ & $78(18.4)$ & 0.33 \\
\hline T-lineage & $65(86.7)$ & $10(13.3)$ & \\
\hline \multicolumn{4}{|l|}{ Treatment arm } \\
\hline Low-risk & $197(82.4)$ & $42(17.6)$ & 1.0 \\
\hline Standard/high-risk & $213(82.2)$ & $46(17.8)$ & \\
\hline
\end{tabular}

${ }^{\mathrm{a}}$ Genetically determined race as described. ${ }^{118}$ bLL immunophenotype. 
(additionally on days 19, 21, 23) in patients with high levels (i.e., 1\% or more) of leukemic cells in bone marrow on day 19 of remission induction.

Patients on the LR arm received 9 doses of Elspar during reinduction I (weeks 7-9 from start of continuation treatment), and 9 doses during reinduction II (weeks 17-19; Figure 2-1). Patients on the SHR arm received Elspar at $25000 \mathrm{U} / \mathrm{m}^{2}$ weekly for 19 doses in continuation treatment (weeks 1-19). For SHR patients with Philadelphia chromosome-positive ALL or induction failure, an additional dose of $25000 \mathrm{U} / \mathrm{m}^{2}$ Elspar was given in the reintensification phase (after consolidation or after reinduction I based on minimal residual disease [MRD] status). Patients with clinical allergy to Elspar were subsequently given Erwinia asparaginase (Erwinase) or PEG-asparaginase (Oncaspar) by i.m. injection, based on their availability (Erwinase was used preferentially when both were available), which was influenced by manufacturer-related drug shortages. Erwinase was given at $20000 \mathrm{U} / \mathrm{m}^{2}$ thrice weekly during remission induction for both LR and SHR patients, $20000 \mathrm{U} / \mathrm{m}^{2}$ thrice weekly during reinduction for LR patients, and $25000 \mathrm{U} / \mathrm{m}^{2}$ twice weekly in weeks 1-19 of continuation therapy for the SHR patients. Oncaspar was given at $2500 \mathrm{U} / \mathrm{m}^{2}$ weekly according to treatment phase. If allergy was confirmed for all three forms, asparaginase was discontinued.

Blood was collected into tubes without anticoagulant for asparaginase measures on days 5, 19, and 34 of remission induction, day 1 of reinduction I, and day 1 of reinduction II. Samples were collected before the asparaginase injection if given on the same day of sampling. Serum was frozen at $-80^{\circ} \mathrm{C}$ until analysis.

\section{Phenotyping of clinical allergy to asparaginase}

The allergic reactions to asparaginase were characterized by local (pain, swelling, erythema) and/or systemic manifestations (fever, urticaria, or edema) and graded using the NCI CTCAE version 3.0; only patients who developed grade 2 or higher reactions received drug substitution. ${ }^{119}$

\section{Anti-asparaginase antibodies}

\section{Antibody assay}

A total of 2010 serum samples (Table 2-2) were measured for antibodies to three forms of asparaginase (Elspar, Erwinase, and Oncaspar). Of these, 471 samples were assayed at the University of Tennessee Health Science Center (UTHSC) and 1345 samples were assayed at SJCRH. Antibodies to three forms of asparaginase (Elspar, Erwinase, and Oncaspar) were measured by ELISA based on a modification of a previously reported assay. ${ }^{120}$ The details are presented in Appendix A (Table A-1 and Figures A-1 through A-3). 


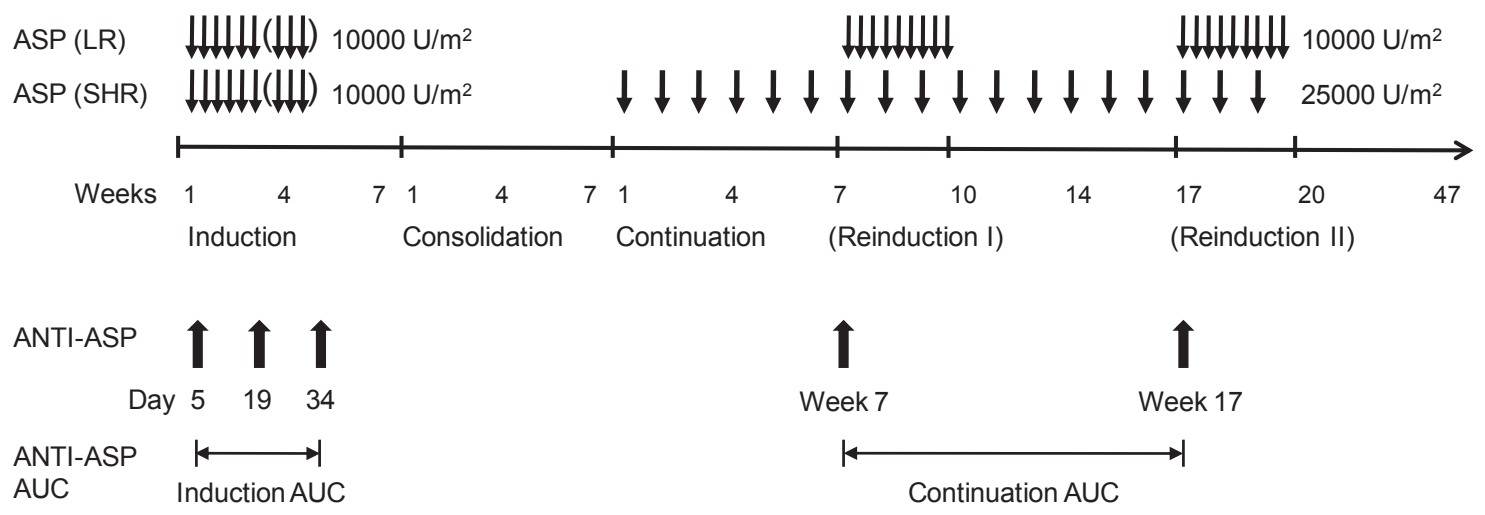

Figure 2-1. Total XV asparaginase regimen and anti-asparaginase antibody measurements

All patients received Elspar (ASP) at $10000 \mathrm{U} / \mathrm{m}^{2}$ per dose during remission induction; patients on the SHR arm received $25000 \mathrm{U} / \mathrm{m}^{2}$ per week at weeks 1-19; those on the LR arm received $10000 \mathrm{U} / \mathrm{m}^{2}$ thrice weekly at reinduction weeks 7-9 and 17-19. Serum samples for anti-asparaginase antibodies (ANTI-ASP) were collected on days 5, 19, and 34 of remission induction and weeks 7 and 17 of continuation therapy. The area under the antibody-concentration-versus-time curve (ANTI-ASP AUC) was estimated for induction (between day 5 and day 34) and continuation (between week 7 and week 17) using OD readings tested against Elspar.

Table 2-2. Number of patients with evaluable blood samples at each time point

\begin{tabular}{lc}
\hline Time point of antibody test & Number of patients (samples) \\
\hline Day 5 of induction & $401(420)$ \\
Day 19 of induction & $392(396)$ \\
Day 34 of induction & $384(399)$ \\
Week 7 of continuation & $381(408)$ \\
Week 17 of continuation & $367(387)$ \\
\hline
\end{tabular}




\section{Data analysis}

Anti-asparaginase antibodies were analyzed as a continuous variable (optical density [OD] readings at 1:400 dilution after normalization across instruments) and also classified as positive or negative. Those with positive antibodies in the absence of clinical allergy were considered to have silent hypersensitivity to asparaginase.

The positive control reference pool was prepared from patients known to have received the specific form of asparaginase and who exhibited clinical signs of allergic reaction and had high titers of antibodies, represented by high OD readings. The negative control reference pool was prepared from healthy volunteers who had never received asparaginase treatment. A sample assayed at SJCRH was considered positive if the natural $\log$ of its 1:400 OD reading was greater than 2.58 standard deviations above the negative control process mean. A sample assayed at UTHSC was considered positive if the natural $\log$ of its 1:3200 OD reading was greater than 2 standard deviations above the negative control process mean. Samples that were not positive were considered negative. Because the magnitudes of the scale of antibody OD readings obtained at the two facilities differed, we normalized the OD readings based on a comparison of 194 serum samples measured for antibody levels at both SJCRH and UTHSC. The normalization of UTHSC OD readings to the same scale was applicable to the SJCRH readings was described by an exponential model using Equation 2-1 (Elspar), Equation 2-2 (Erwinase), and Equation 2-3 (Oncaspar) to maintain consistency of OD readings between UTHSC and SJCRH. All of the samples measured at UTHSC had their OD readings normalized to the scale of the readings obtained at SJCRH. The details of data normalization are shown in Appendix A.

$\log ($ normalized OD $)=-5.066+3.509 \cdot \exp [0.526 \times \log ($ original OD $)]$

$\log ($ normalized OD $)=-4.360+3.518 \cdot \exp [0.806 \times \log ($ original OD $)]$

$\log ($ normalized OD $)=-5.280+3.684 \cdot \exp [0.520 \times \log ($ original OD $)]$

\section{Estimation of antibody area-under-the-curve (AUC)}

To estimate the long-term exposure to antibodies, the area under the antibodyconcentration-versus-time curve (AUC) was estimated using the method of trapezoids in the 360 patients who had at least four out of five scheduled antibody tests over the first 35 weeks of therapy (including week 7 and week 17 tests in the continuation phase and at least two antibody tests in the induction phase). Induction AUC (antibody AUC between day 5 and day 34 of remission induction) and continuation AUC (antibody AUC between week 7 and week 17 of continuation) were estimated separately using OD readings for each patient's serum tested against Elspar, the front-line asparaginase preparation.Analyses were performed using the sum of the induction AUC and continuation AUC to Elspar (Figure 2-1). 
Patients were considered to have detectable antibody if the AUC was greater than 2.12 (OD value $\times$ days), which corresponds to 2.58 standard deviations above the mean value of negative cases (i.e., the antibody AUCs of the patients negative for anti-Elspar antibody consistently during therapy; Figure A-4).

\section{Asparaginase activity}

Serum asparaginase activity was determined in a subset of samples from patients in the SHR arm: 77 week-7 samples and 69 week-17 samples which were collected 6-8 days after the last Elspar administration of $25000 \mathrm{U} / \mathrm{m}^{2}$; 9 week-7 samples and 10 week17 samples which were collected 3-4 days after the last Erwinase administration of 25000 $\mathrm{U} / \mathrm{m}^{2}$; and 14 week-7 samples and 16 week-17 samples which were collected 6-8 days after the last Oncaspar administration of $2500 \mathrm{U} / \mathrm{m}^{2}$.

We used a kinetic spectrophotometric assay similar to a previously reported assay, ${ }^{32}$ with a faster and more controlled process as well as improved linearity range without sample pretreatments. The asparaginase activity in serum was measured by an enzymatically coupled oxidation of reduced nicotinamide adenine dinucleotide (NADH). The reactions were shown in Figure 2-2. When monitored by spectrophotometry, the oxidation of NADH leads to a corresponding decrease in absorbance at $340 \mathrm{nM}$. The rate of change of absorbance is directly proportional to asparaginase activity.

The enzyme reaction mixture was prepared by combining $200 \mathrm{~mL}$ of 10x Trisbuffered saline, $400 \mathrm{~mL}$ of glycerol, $200 \mathrm{mg}$ of $\alpha$-ketoglutaric acid, $264 \mathrm{mg}$ of asparagine, $200 \mathrm{mg}$ of $\beta$-NADH, $400 \mu \mathrm{L}$ of glutamic oxaloacetic transaminase, $200 \mu \mathrm{L}$ of malic dehydrogenase, and $1400 \mathrm{~mL}$ distilled and deionized water, and stored at $-80^{\circ} \mathrm{C}$. (All the reagents above were from Sigma-Aldrich, St. Louis, MO.) Before plate reading, the enzyme reaction mixture was incubated in a $37^{\circ} \mathrm{C}$ water bath for $30 \mathrm{~min}$. Then $10 \mu \mathrm{L}$ patient sera were added to 96-well UV-transparent plates (Corning. Inc, NY) in quadruplicate.

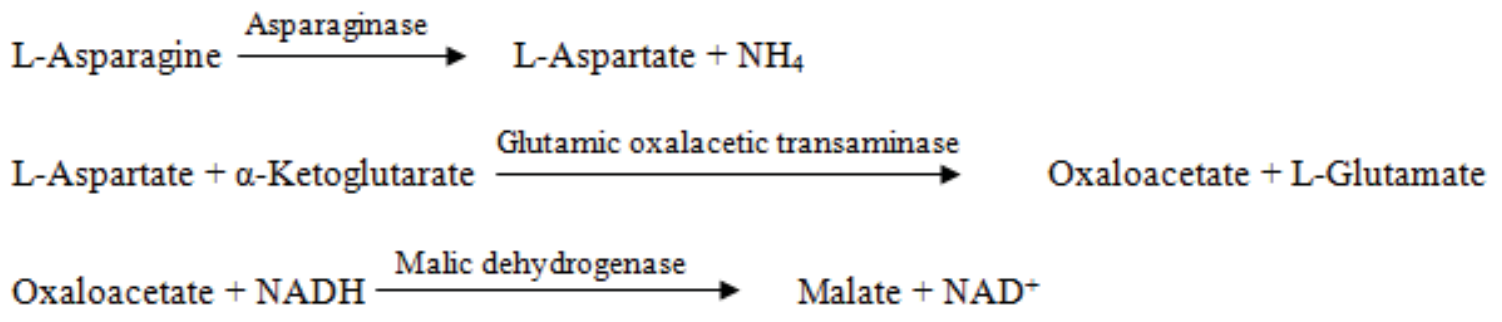

Figure 2-2. Enzyme reactions in asparaginase activity assay 
The reaction was started by adding $190 \mu \mathrm{L}$ of enzyme reaction mixture to each well. The enzyme reaction mixture was added one row at a time, with a 20 second interval between each addition. The plate was read using a BioTek ELx808IU Microplate Reader (BioTek, Winooski, VT) at $340 \mathrm{~nm}$ with a 20 second interval for $15 \mathrm{~min}$. The activity was estimated by the slope of the change of optical density over time.The linearity range of this assay was $0.011-2.2 \mathrm{U} / \mathrm{mL}$. Samples higher than $2.2 \mathrm{U} / \mathrm{mL}$ were diluted with sample dilution buffer (5\% bovine serum albumin in deionized water). Calibrators were established with asparaginase standard solutions: $0.025-2.0 \mathrm{U} / \mathrm{mL}$ native E. coli asparaginase (BioVendor Laboratory Medicine. Inc, Czech Republic) in sample dilution buffer.

\section{Ex vivo asparaginase neutralization assay}

Patient serum samples were tested for their neutralizing effect against asparaginase using an ex vivo neutralization assay. Elspar and Oncaspar were diluted with sample dilution buffer ( $5 \%$ bovine serum albumin in deionized water) to $1.0 \mathrm{IU} / \mathrm{mL}$, and then mixed with patient sera at $1: 1$ volume. The mixture was incubated at $37^{\circ} \mathrm{C}$ for 1 hour. The remaining asparaginase activity was then measured as described above.

\section{Prognostic value of antibody tests}

\section{Sensitivity, specificity and predictive values}

Sensitivity, specificity, positive predictive value (PPV) and negative predictive value (NPV) were calculated using the numbers of patients with true positive (TP), false positive (FP), true negative (TN) and false negative (FN) antibody results. For this purpose, patients who had clinical reactions attributed to Elspar over a given time period (e.g., weeks 7-9) were classified as TP or FN, depending on whether they were positive for anti-Elspar at an adjacent time point (e.g., week 7); the patients who never had a clinical reaction were classified as TN or FP, depending on whether they were ever positive for anti-Elspar during therapy. Sensitivity was defined as the percentage of reacting patients who were positive for anti-Elspar (calculated as TP / [TP + FN]); specificity was defined as the percentage of non-reacting patients who were negative for anti-Elspar (calculated as TN / [TN + FP]); PPV was defined as the likelihood that a patient who was positive for antibody had a clinical reaction (calculated as TP / [TP + FP]); and NPV was defined as the likelihood that a patient who was negative for antibody never had a clinical reaction (calculated as TN / [TN + FN]).

\section{Receiver-operator-characteristic (ROC) curves}

The prognostic capacity of antibody tests at different threshold levels were also evaluated in terms of the ROC curve. The ROC curves of antibody tests were generated 
by plotting the true positive rate (sensitivity) and corresponding false positive rate (1specificity) for every possible threshold OD value (to separate positive from negative samples). The AUC of the ROC curve is a relative measure of the diagnostic accuracy of a test. An AUC of 1.0 would indicate a perfect test (100\% TP, 0\% FP, $100 \%$ TN, $0 \%$ FN), whereas 0.5 would indicate a random classification. A value greater 0.9 represents a rather high accuracy, a value less than 0.7 represents a low accuracy, and a value in between would be considered fair to good. ${ }^{121}$

\section{Asparaginase adverse effects in Total XV}

Other than allergy, the adverse effects possibly related to asparaginase include pancreatitis, thrombosis, and osteonecrosis, which exposure were uniformly assessed in all patients. Patients were prospectively screened by magnetic resonance imaging (MRI) for osteonecrosis of the hips and knees, which was graded as 0 (absent), 1 (asymptomatic), 2 (symptomatic), 3 (severe), and 4 (disabling) based on the NCI CTCAE version 3.0, as previously reported. ${ }^{90}$ All cases of grade 2-4 thrombosis and pancreatitis before week 20 of continuation therapy were considered possible adverse effects of asparaginase.

\section{Statistical analysis}

Statistical analyses were conducted using R 2.11.1 (R Development Core Team, http://www.r-project.org) and SAS 9.2 (SAS Institute Inc., Cary, NC, USA).

Comparisons of continuous variables between groups were conducted with Wilcoxon rank sum test. Fisher's exact test was used to determine the difference in frequencies of categorical variables (e.g., allergy or antibody positivity). Nonlinear and linear regression analyses were used to describe the change of antibody OD readings with time or with the frequency of clinical reaction. Recursive partitioning approach (using with the rpart function of R) was performed to determine the optimal threshold to differentiate allergy status based on OD values. Logistic regression was applied for multivariate analysis of risk factors of osteonecrosis; and the cumulative incidence of osteonecrosis was compared between groups by log-rank test. Pancreatitis and thrombosis were compared between groups using time-dependent analysis, ${ }^{122}$ a_Cox proportional-hazards regression model where antibody status was treated as a time-dependent covariate.

\section{Results}

\section{Asparaginase allergy}

Among 410 patients, 169 (41\%) had clinical allergy to Elspar, of whom 72 were first re-challenged with Erwinase and 94 with Oncaspar, and 3 were switched to other drugs. Thirty-three patients experienced a second episode of allergy (9 to Erwinase and 
24 to Oncaspar), and 2 patients developed allergy to all three preparations. Patients in the LR arm were more likely than those in the SHR arm to experience hypersensitivity to Elspar, with cumulative incidences of clinical allergy being $51 \%$ versus $32 \%(P=$ 0.0002 ), a finding consistent with that of our prior report. ${ }^{119}$ Antibody against Elspar was also more common in patients in the LR arm than the SHR arm: $69 \%$ versus $47 \%, P=$ $6.6 \times 10^{-6}($ Figure 2-3).

The majority of allergic reactions occurred upon first re-exposure to Elspar following a 10- to 16-week hiatus after the induction phase: 78\% (54 of 69) of patients in the SHR arm who had clinical allergy reacted during continuation weeks 1-6, and 96\% (96 of 100) of patients in the LR arm who had clinical allergy reacted at reinduction I (Table 2-3).

We also investigated whether other clinical features such as age, gender, race, or ALL lineage were associated with the risk of developing antibodies or an allergic reaction to Elspar (Table 2-4 and Table 2-5). Age and gender were not associated with allergy or antibody status; age was not related to allergy $(P=0.68)$ or antibody status $(P$ $=0.86$ ) even when the analysis was restricted to those with B-lineage ALL (adjusting for treatment arm and race). Allergies differed by race: reactions occurred in $45 \%$ of white, $31 \%$ of black, and $33 \%$ of other (Hispanic and Asian) patients $(P=0.037)$, and the frequencies of positive antibodies during therapy were $62 \%, 55 \%$, and $40 \%$ in those patient groups $(P=0.005)$, respectively. All patients with T-cell ALL were enrolled in the SHR arm, and they had significantly fewer clinical reactions (19\% vs. $39 \%, P=$ $0.003)$ and lower antibodies ( $35 \%$ vs. $52 \%, P=0.02)$ to Elspar than did those with Blineage ALL in the SHR arm.

\section{Antibodies and allergy}

Anti-Elspar antibodies were more frequent among the patients with clinical allergy to Elspar: $87.0 \%$ (147 of 169 reacting patients) compared to only $36.9 \%$ (89 of $241)$ among non-reacting patients $\left(P<1.0 \times 10^{-15}\right)$. Among the 236 patients who were considered positive for anti-Elspar antibody, those who had reactions had higher levels of antibody (based on OD readings) at the end of induction $(P=0.0002)$ and during the continuation phase (week 7, $P=8 \times 10^{-9}$; and week 17, $P<1.0 \times 10^{-15}$ ), and higher cumulative antibody titer based on anti-Elspar antibody AUC (27.8 \pm 17.9 vs. $10.8 \pm$ 13.3, $P<1 \times 10^{-15}$ ) than those who did not have reactions (i.e., the group who would be classified as "silent hypersensitivity"; Figure 2-4). However, patients with more severe clinical toxicity (grade 3-4 reactions) against Elspar did not have higher cumulative antiElspar antibody AUC $(22.8 \pm 23.0$ vs. $28.6 \pm 16.9, P=0.11)$ than those with mild toxicity (grade 2 reactions). For Oncaspar, similarly, we observed no difference in antiOncaspar antibody AUC in those with grade 3-4 vs. grade 2 reactions (5.7 \pm 7.8 vs. $5.4 \pm$ $4.8, P=0.83)$. For Erwinase, all allergic reactions were grade 2, and thus no comparisons are possible. 


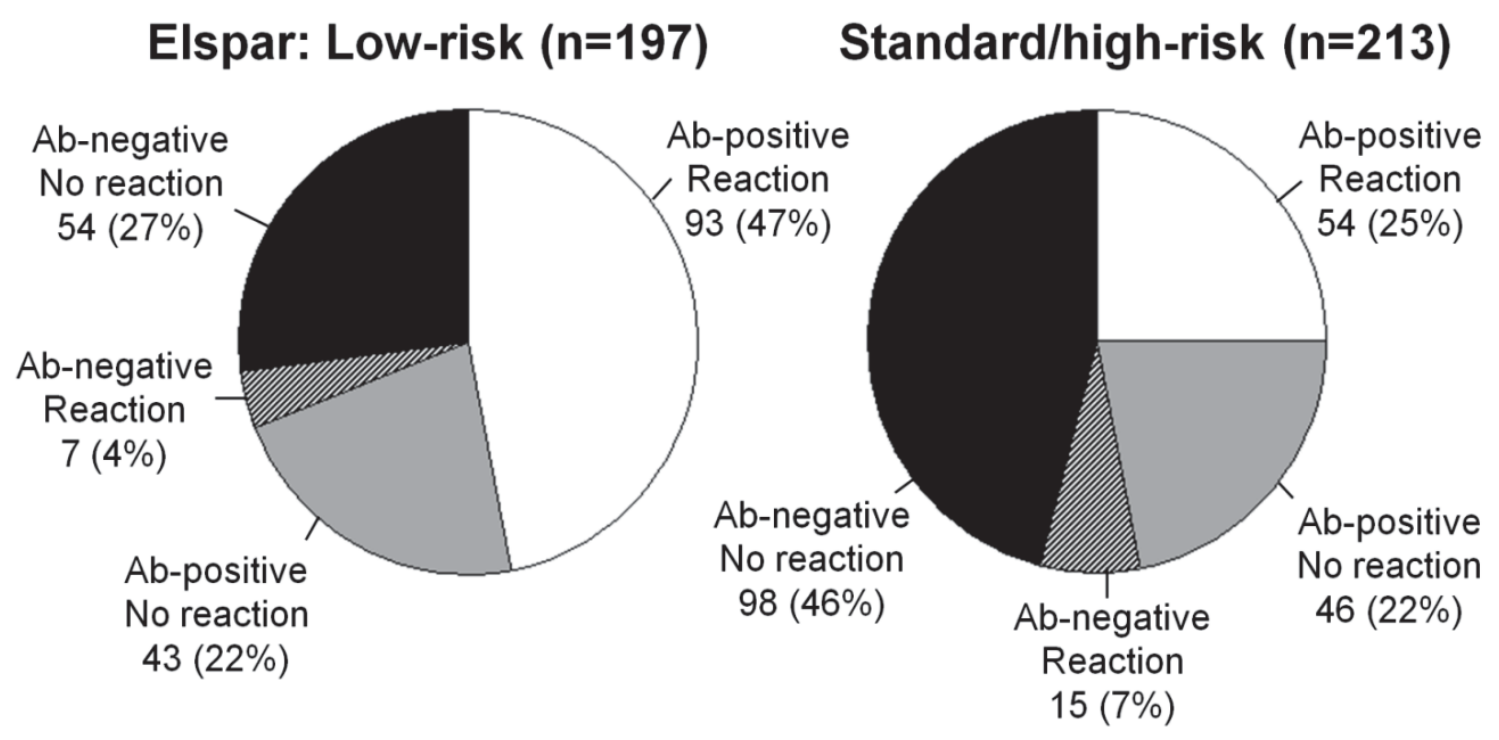

Figure 2-3. Frequency of hypersensitivity to Elspar

The frequency of allergic reactions and anti-Elspar antibody $(\mathrm{Ab})$ positivity in patients enrolled on the low-risk (LR) and standard/high-risk (SHR) treatment arms. Compared with those on the SHR arm, patients on the LR arm had more reactions $(P<0.00001)$ and anti-Elspar antibody $(P=0.0002)$.

Table 2-3. Timing of allergic reaction to Elspar

\begin{tabular}{lccc}
\hline Phase & $\begin{array}{c}\text { Low-risk } \\
(\mathbf{n}=\mathbf{1 0 0})\end{array}$ & $\begin{array}{c}\text { Standard/high-risk } \\
(\mathbf{n}=\mathbf{6 9})\end{array}$ & $\begin{array}{c}\text { Total } \\
(\mathbf{n}=\mathbf{1 6 9})\end{array}$ \\
\hline Induction & $2(2 \%)$ & $3(4 \%)$ & $5(3 \%)$ \\
Continuation weeks 1-6 & $0(0 \%)$ & $54(78 \%)$ & $54(32 \%)$ \\
Reinduction I & $96(96 \%)$ & $1(2 \%)$ & $97(58 \%)$ \\
Continuation weeks 10-16 & $0(0 \%)$ & $7(10 \%)$ & $7(4 \%)$ \\
Reinduction II & $2(2 \%)$ & $0(0 \%)$ & $2(1 \%)$ \\
Reintensification & $0(0 \%)$ & $4(6 \%)$ & $4(2 \%)$ \\
\hline
\end{tabular}

Number (and percentage) of patients reacting to Elspar at each phase of therapy are compared to the total number of patients who had a reaction by risk groups. 
Table 2-4. Clinical features and allergic reactions to Elspar

\begin{tabular}{|c|c|c|c|c|c|c|c|c|c|c|c|c|}
\hline \multirow[b]{2}{*}{$\begin{array}{l}\text { Clinical } \\
\text { features }\end{array}$} & \multicolumn{4}{|c|}{ All patients $(n=410)^{\mathrm{a}}$} & \multicolumn{4}{|c|}{ Low-risk $\operatorname{arm}(\mathrm{n}=197)$} & \multicolumn{4}{|c|}{ Standard/high-risk $\operatorname{arm}(n=213)$} \\
\hline & $\mathbf{n}$ & $\begin{array}{c}\text { Allergy } \\
\text { n (\%) }\end{array}$ & $\begin{array}{c}\text { No allergy } \\
\text { n }(\%)\end{array}$ & $\boldsymbol{P}$ & $\mathbf{n}$ & $\begin{array}{c}\text { Allergy } \\
\text { n (\%) }\end{array}$ & $\begin{array}{c}\text { No allergy } \\
\text { n }(\%)\end{array}$ & $\boldsymbol{P}$ & $\mathbf{n}$ & $\begin{array}{c}\text { Allergy } \\
\text { n (\%) }\end{array}$ & $\begin{array}{c}\text { No allergy } \\
\text { n }(\%)\end{array}$ & $\boldsymbol{P}$ \\
\hline \multicolumn{13}{|l|}{ Age } \\
\hline$<10$ years & 302 & $129(42.7)$ & $173(57.3)$ & 0.36 & 183 & $94(51.4)$ & $89(48.6)$ & 0.59 & 119 & $35(29.4)$ & $84(70.6)$ & 0.31 \\
\hline$\geq 10$ years & 108 & $40(37.0)$ & $68(63.0)$ & & 14 & $6(42.9)$ & $8(57.1)$ & & 94 & $34(36.2)$ & $60(63.8)$ & \\
\hline \multicolumn{13}{|l|}{ Sex } \\
\hline Male & 228 & $96(42.1)$ & $132(57.9)$ & 0.69 & 93 & $53(57.0)$ & $40(43.0)$ & 0.12 & 135 & $43(31.9)$ & $92(68.1)$ & 0.88 \\
\hline Female & 182 & $73(40.1)$ & $109(59.9)$ & & 104 & $47(45.2)$ & $57(54.8)$ & & 78 & $26(33.3)$ & $52(66.7)$ & \\
\hline \multicolumn{13}{|l|}{$\operatorname{Race}^{\mathrm{b}}$} \\
\hline White & 282 & $128(45.4)$ & $154(54.6)$ & 0.037 & 146 & $75(51.4)$ & $71(48.6)$ & 0.19 & 136 & $53(39.0)$ & $83(61.0)$ & 0.004 \\
\hline Black & 65 & $20(30.8)$ & $45(69.2)$ & & 24 & $15(62.5)$ & $9(37.5)$ & & 41 & $5(12.2)$ & $36(87.8)$ & \\
\hline Other & 63 & $21(33.3)$ & $42(66.7)$ & & 27 & $10(37.0)$ & $17(63.0)$ & & 36 & $11(30.6)$ & $25(69.4)$ & \\
\hline \multicolumn{13}{|c|}{ Immunophenotype $^{c}$} \\
\hline B-lineage & 345 & $157(45.5)$ & $188(54.5)$ & 0.0001 & 197 & $100(50.8)$ & $97(49.2)$ & NA & 148 & $57(39.2)$ & $91(60.8)$ & 0.003 \\
\hline T-lineage & 65 & $12(18.5)$ & $53(81.5)$ & & 0 & $0(0.0)$ & $0(0.0)$ & & 65 & $12(18.5)$ & $53(81.5)$ & \\
\hline
\end{tabular}

${ }^{a}$ Patients with serum samples evaluable for anti-asparaginase antibodies $(n=410)$. Analysis for all patients $(n=498)$ has been previously reported. ${ }^{119 \mathrm{~b}}$ Genetically determined race as described. ${ }^{118 \mathrm{c}}$ Acute lymphoblastic leukemia immunophenotype. NA, not applicable. 
Table 2-5. Clinical features and anti-Elspar antibody status

\begin{tabular}{|c|c|c|c|c|c|c|c|c|c|c|c|c|}
\hline \multirow[b]{2}{*}{$\begin{array}{l}\text { Clinical } \\
\text { features }\end{array}$} & \multicolumn{4}{|c|}{ All patients $(n=410)$} & \multicolumn{4}{|c|}{ Low-risk arm $(n=197)$} & \multicolumn{4}{|c|}{ Standard/high-risk arm $(n=213)$} \\
\hline & $\mathbf{n}$ & $\begin{array}{c}\text { Positive } \\
\text { n (\%) }\end{array}$ & $\begin{array}{c}\text { Negative } \\
\text { n }(\%)\end{array}$ & $P$ & n & $\begin{array}{c}\text { Positive } \\
\text { n (\%) }\end{array}$ & $\begin{array}{c}\text { Negative } \\
\text { n }(\%)\end{array}$ & $P$ & n & $\begin{array}{c}\text { Positive } \\
\text { n }(\%)\end{array}$ & $\begin{array}{c}\text { Negative } \\
\text { n (\%) }\end{array}$ & $P$ \\
\hline \multicolumn{13}{|l|}{ Age } \\
\hline$<10$ years & 302 & $182(60.3)$ & $120(39.7)$ & 0.07 & 183 & $129(70.5)$ & $54(29.5)$ & 0.14 & 119 & $53(44.5)$ & $66(55.5)$ & 0.49 \\
\hline$\geq 10$ years & 108 & $54(50.0)$ & $54(50.0)$ & & 14 & $7(50.0)$ & $7(50.0)$ & & 94 & $47(50.0)$ & $47(50.0)$ & \\
\hline \multicolumn{13}{|l|}{ Sex } \\
\hline Male & 228 & $135(59.2)$ & $93(40.8)$ & 0.48 & 93 & $70(75.3)$ & $23(24.7)$ & 0.09 & 135 & $65(48.2)$ & $70(51.8)$ & 0.67 \\
\hline Female & 182 & $101(55.5)$ & $81(44.5)$ & & 104 & $66(63.5)$ & $38(36.5)$ & & 78 & $35(44.9)$ & $43(55.1)$ & \\
\hline \multicolumn{13}{|l|}{ Race $^{\mathrm{a}}$} \\
\hline White & 282 & $175(62.1)$ & $107(37.9)$ & 0.005 & 146 & $104(71.2)$ & $42(28.8)$ & 0.40 & 136 & $71(52.2)$ & $65(47.8)$ & 0.013 \\
\hline Black & 65 & $36(55.4)$ & $29(44.6)$ & & 24 & $16(66.7)$ & $8(33.3)$ & & 41 & $20(48.8)$ & $21(51.2)$ & \\
\hline Other & 63 & $25(39.7)$ & $38(60.3)$ & & 27 & $16(59.3)$ & $11(40.7)$ & & 36 & $9(25.0)$ & $27(75.0)$ & \\
\hline \multicolumn{13}{|c|}{ Immunophenotype ${ }^{b}$} \\
\hline B-lineage & 345 & $213(61.7)$ & $132(38.3)$ & 0.0001 & 197 & $136(69.0)$ & $61(31.0)$ & NA & 148 & $77(52.0)$ & $71(48.0)$ & 0.026 \\
\hline $\mathrm{T}$-lineage & 65 & $23(35.4)$ & $42(64.6)$ & & 0 & $0(0.0)$ & $0(0.0)$ & & 65 & $23(35.4)$ & $42(64.6)$ & \\
\hline
\end{tabular}

${ }^{\mathrm{a}}$ Genetically determined race as described. ${ }^{118}$ b Acute lymphoblastic leukemia immunophenotype. NA, not applicable. 


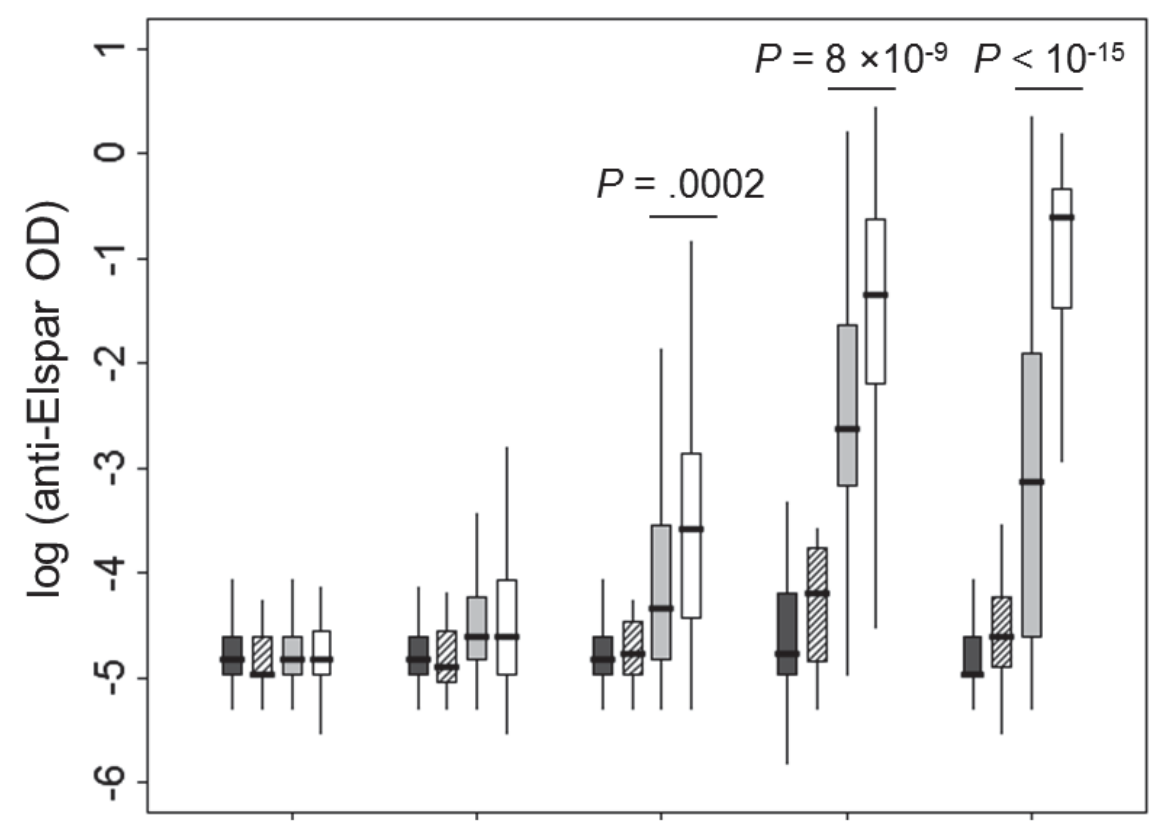

Day 5 Day 19 Day 34 Week 7 Week 17

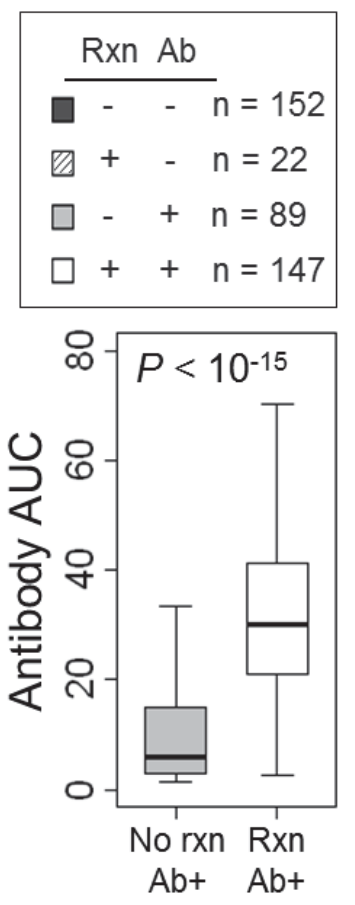

Figure 2-4. Antibody levels in patients with and without clinical reactions Left: Anti-Elspar optical density (OD) measures (natural log) for 410 patients at days 5, 19 , and 34 of remission induction and weeks 7 and 17 of continuation therapy. At each time point, patients are grouped based on whether they ever (or never) experienced a clinical allergic reaction (Rxn), and whether they were ever (or never) positive for antiElspar antibody $(\mathrm{Ab})$ at anytime during therapy. Among the patients positive for antiElspar, those who had reactions (white bars) had significantly higher anti-Elspar levels than did those who had silent hypersensitivity (light grey) at day 34, week 7, and week 17. Among the patients negative for anti-Elspar antibodies (as defined by the threshold in the Supplement), those who had allergic reactions (hatched) exhibited slightly elevated anti-Elspar levels compared with those without any allergic reactions (dark grey) at weeks $7(P=0.05)$ and $17(P=0.03)$. Right: cumulative anti-Elspar antibody AUC over the first 35 weeks of therapy for antibody-positive patients who had allergic reactions versus those who had silent hypersensitivity. Box plots: median, quartiles, non-outlier range. 
This study's sampling strategy was designed to have evaluable antibody samples at comparable times relative to asparaginase dosing for all patients (those who developed an allergy and those who did not). Because allergic reactions may have occurred at times during which no samples were obtained, maximal values may not have been captured if antibody levels are maximal at the time of reaction. However, serial samples that happened to be obtained before, during, and after reactions indicated that antibody levels were estimated to be maximal at about 50 days after an allergic reaction (Figure 2-5).

We investigated the utility of serum anti-Elspar antibody positivity to predict future (if sampling just before) or to confirm (if sampling just after) clinical allergic reactions. The sensitivity, specificity, and predictive values are shown in Table 2-6. The greatest utility was evident for the week 7 serum samples, which happened to be obtained closest to the time period during which most reacting patients had their allergic reaction (continuation weeks 7-9 for those in the LR arm and weeks 1-6 for those in the SHR arm). The week 7 anti-Elspar antibody test had a sensitivity of $87-88 \%$ and a specificity of $68-69 \%$ for predicting clinical reactions to Elspar at weeks 7-9 for LR patients and for confirming clinical reactions to Elspar at weeks 1-6 for SHR patients. Using the optimal OD thresholds determined by recursive partitioning (Table A-2 and Figure A-5), the specificity was slightly improved to $71-74 \%$ but the sensitivity of prediction was lowered to $83 \%$. The prognostic utility of the antibody tests at day 34, week 7 and week 17, as evaluated by the AUC of the ROC curves, ranged from 0.70 to 0.91 (Figure 2-6).

Antibody test obtained at day 34 of induction for future clinical reactions showed a lower prognostic capacity compared with those at week 7 and week 17 (Figure 2-6).

Interestingly, we observed a strong positive linear relationship between the antiElspar level (natural log of OD reading) at week 7 and the probability of patients reacting to Elspar at weeks 7-9 on the LR arm (R-squared $\left.=0.96, P=6 \times 10^{-8}\right)$ or weeks 1-6 on the SHR arm (R-squared $=0.83, P=4 \times 10^{-5}$; Figure 2-7), consistent with our finding that anti-Elspar level was higher in patients with allergy than those with silent hypersensitivity (Figure 2-4).

The frequency of secondary allergy to Oncaspar was higher than to Erwinase ( $24 / 94$ or $26 \%$ vs. $9 / 72$ or $13 \%, P=0.05$ ), probably due to the cross reactivity between Oncaspar and Elspar (Figure A-6). For secondary treatment with Erwinase and Oncaspar, there was less association between clinical reactions to these agents and corresponding antibodies. We measured anti-Erwinase and anti-Oncaspar antibodies in 63 of the 72 patients who went on to be treated with Erwinase and 85 of the 94 patients who went on to receive Oncaspar. Among the 63 patients receiving Erwinase, 9 had an allergy to it, of whom only 4 had detectable anti-Erwinase antibody. Among the 85 patients receiving Oncaspar, 23 had a clinical reaction, of whom 20 had detectable antiOncaspar antibody. In addition, silent hypersensitivity occurred among 8 of 63 (13\%) patients treated with Erwinase and among 47 of 85 (55\%) treated with Oncaspar (Figure 2-8). 


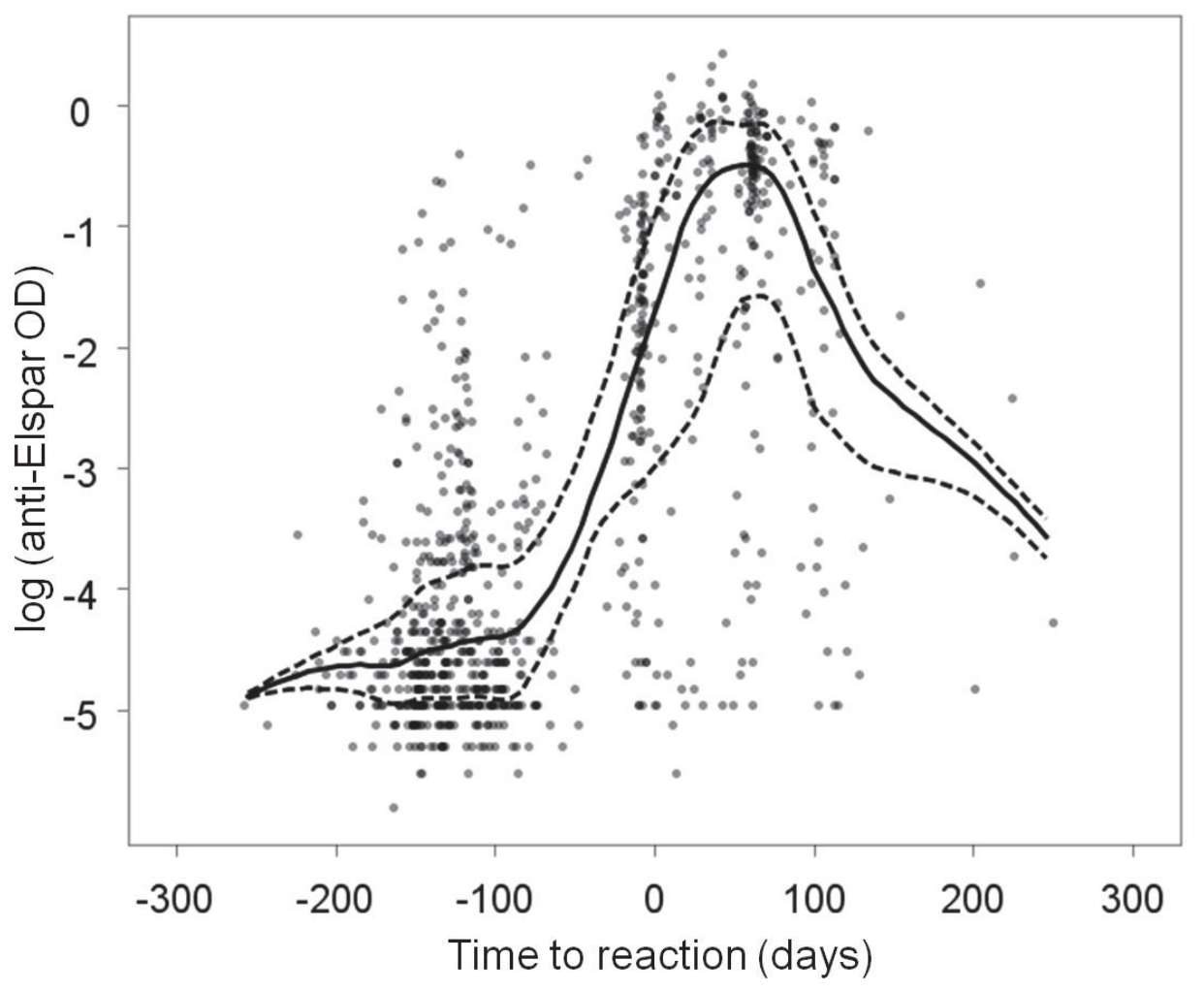

Figure 2-5. Anti-Elspar antibody level relative to the time of clinical reaction to Elspar

Anti-Elspar OD level in patient samples (dots) relative to the number of days elapsed before (negative) or after (positive) between sample collection and clinical reaction to Elspar. The trend lines of median (solid) and quartiles (dashed) are shown. 
Table 2-6. Sensitivity, specificity and predictive values of three anti-Elspar antibody tests for predicting or confirming clinical reactions to Elspar

\begin{tabular}{|c|c|c|c|c|c|c|c|c|}
\hline Risk arm & $\begin{array}{c}\mathrm{N} \text { of } \\
\text { patients }\end{array}$ & $\begin{array}{l}\text { Time of } \\
\text { Ab test }\end{array}$ & $\begin{array}{l}\text { Time of } \\
\text { Rxn }\end{array}$ & $\begin{array}{c}\text { Time between } \\
\text { test and rxn } \\
\text { (weeks) }\end{array}$ & $\begin{array}{c}\text { Sensitivity } \\
(\%)\end{array}$ & $\begin{array}{c}\text { Specificity } \\
(\%)\end{array}$ & $\begin{array}{l}\text { PPV } \\
(\%)\end{array}$ & $\begin{array}{l}\text { NPV } \\
(\%)\end{array}$ \\
\hline \multicolumn{9}{|c|}{ Prediction of future reactions } \\
\hline LR & 185 & Day 34 & Weeks 7-9 & $17-20$ & 42.8 & 92.5 & 84.8 & 62.6 \\
\hline SHR & 190 & Day 34 & Weeks 1-6 & $10-17$ & 26.1 & 93.1 & 54.5 & 79.8 \\
\hline LR & 192 & Week 7 & Weeks 7-9 & $0-3$ & 87.4 & 68.0 & 72.8 & 84.6 \\
\hline \multicolumn{9}{|c|}{ Confirmation of past reactions } \\
\hline SHR & 184 & Week 7 & Weeks 1-6 & $0-6$ & 87.8 & 68.9 & 50.6 & 93.9 \\
\hline LR & 189 & Week 17 & Weeks 7-9 & $7-10$ & 89.4 & 65.3 & 71.8 & 86.1 \\
\hline SHR & 174 & Week 17 & Weeks 1-16 & $0-16$ & 72.7 & 88.2 & 74.1 & 87.5 \\
\hline
\end{tabular}

SHR, standard/high-risk; LR, low-risk; PPV, positive predictive value; NPV, negative predictive value; Ab, anti-Elspar antibody; Rxn, clinical allergic reaction; Day 34, day 34 of remission induction therapy (34 days from diagnosis of ALL); Week 7, week 7 of continuation therapy (23 weeks from diagnosis of ALL; and Week 17, week 17 of continuation therapy (33 weeks from diagnosis of ALL). 


\section{Figure 2-6. ROC curves of the antibody tests}

Receiver operating characteristic (ROC) curves of the antibody tests at day 34, week 7 and week 17, showing the true positive rates and respective false positive rates for predicting or confirming clinical allergic reactions. Each point on the curve corresponds to a specific threshold of anti-Elspar OD that putatively divides antibody-positive from antibody-negative samples. Right $y$-axis shows the color key that visualizes the mapping from the natural log of anti-Elspar OD to colors. The positive threshold dividing positive from negative samples for logOD is -3.2 (circles). The AUC of the ROC curves, which represents overall test performance on predictions, is shown in each graph. LR, low-risk; SHR, standard/high-risk; Ab, antiElspar antibody; Rxn, clinical reaction; AUC, area under the curve. Time of antibody tests: D34, Day 34 of induction therapy; W7, Week 7 of continuation therapy; and W17, Week 17 of continuation therapy. 
D34 Ab vs. W7-9 Rxn (LR)

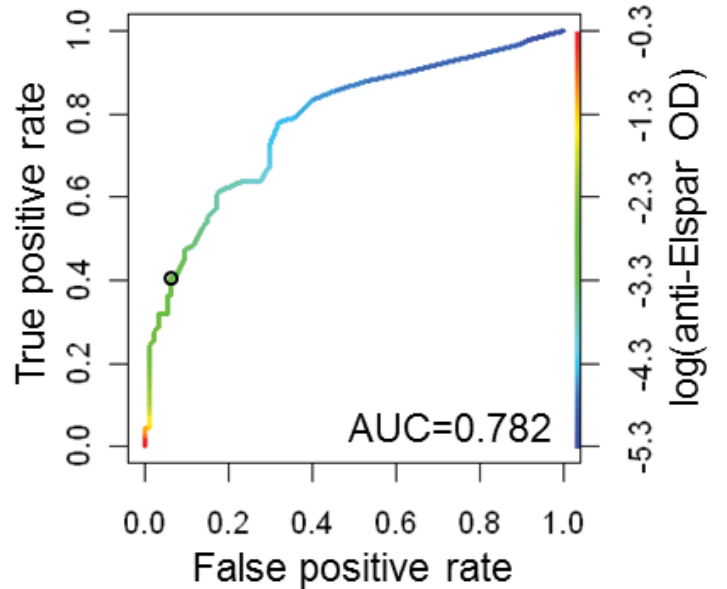

D34 Ab vs. W1-6 Rxn (SHR)

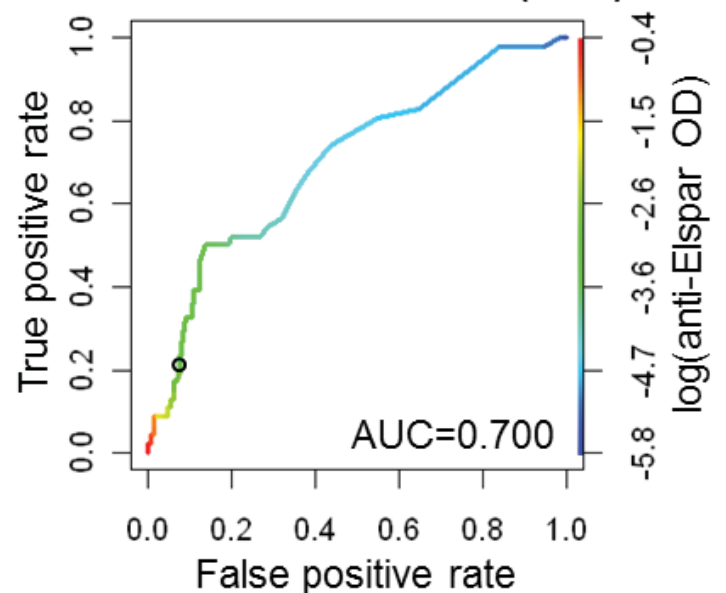

W7 Ab vs. W7-9 Rxn (LR)

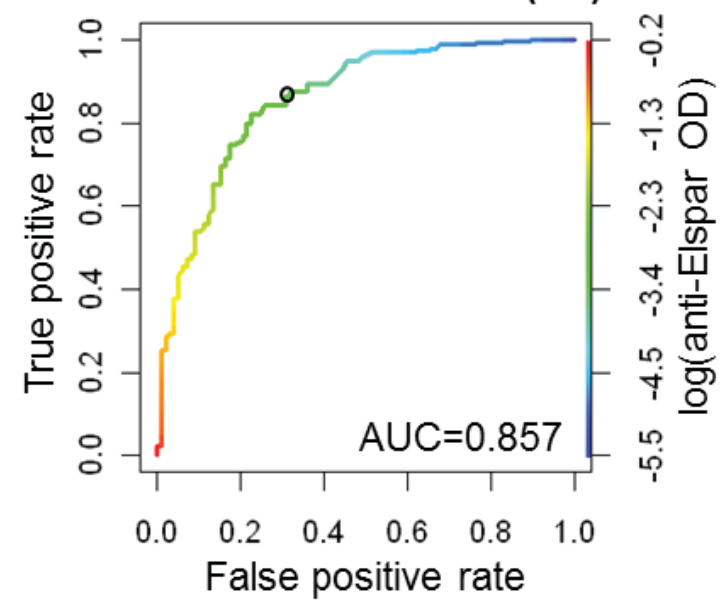

W7 Ab vs. W1-6 Rxn (SHR)

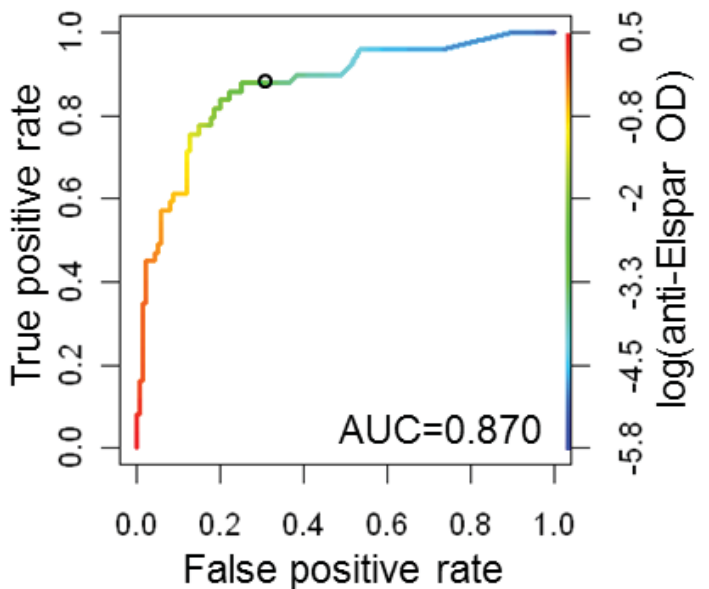

W17 Ab vs. W7-9 Rxn (LR)

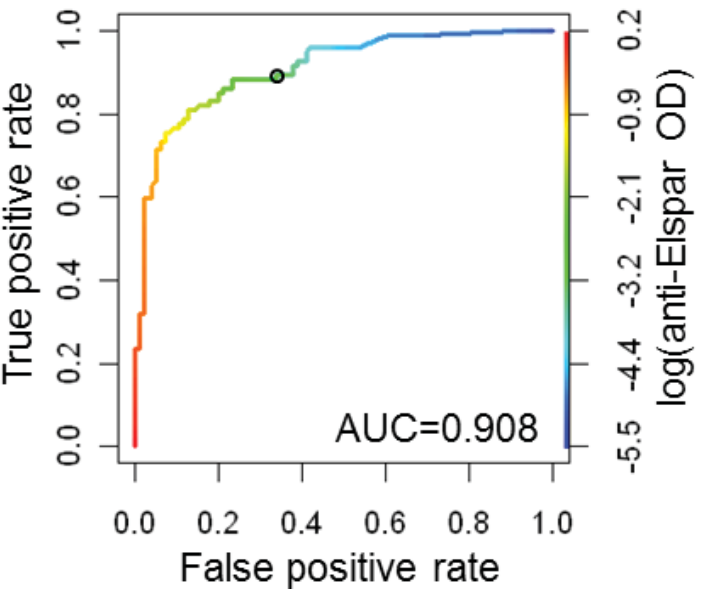

W17 Ab vs. W1-16 Rxn (SHR)

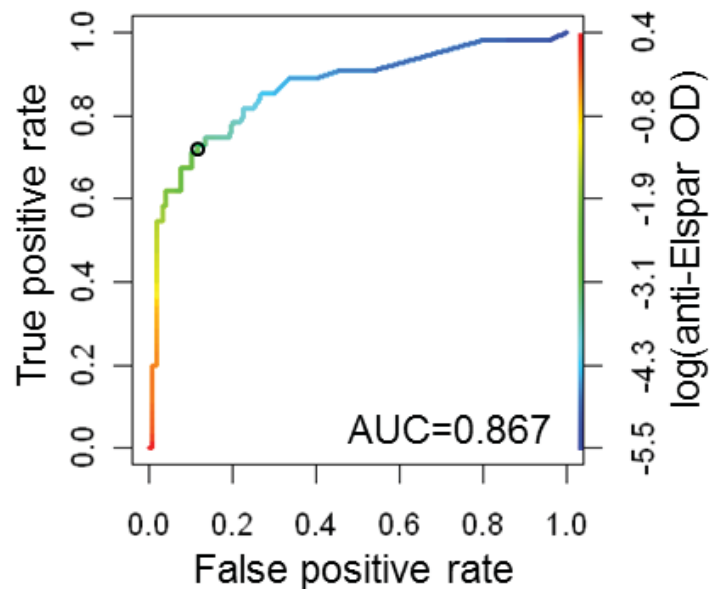



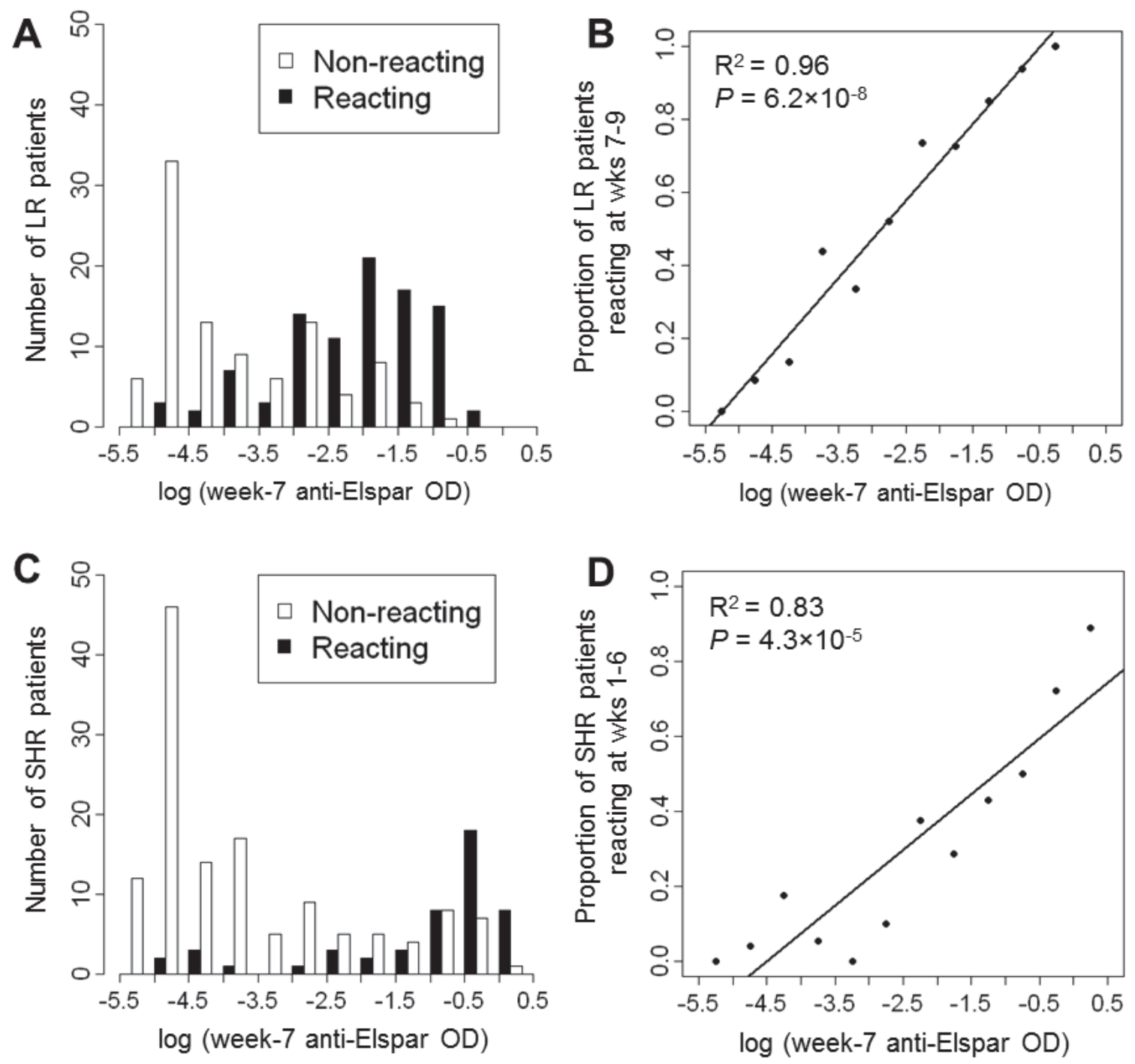

Figure 2-7. Association between week 7 anti-Elspar antibody level and the proportion of patients reacting to Elspar around week 7

Left panels: the distribution of patients with (solid bars) and without (open bars) clinical reactions by their anti-Elspar OD at week 7. (A) Patients on the LR arm $(n=195)$ with and without reactions during weeks 7-9. (C) Patients on the SHR arm ( $n=175)$ with and without reactions during weeks 1-6. Right panels: the positive correlation between week 7 anti-Elspar OD and the proportion of patients having clinical reaction at (B) weeks 7-9 (LR) and (D) weeks 1-6 (SHR). Trend lines are shown. LR, low-risk; SHR, standard/high-risk. 


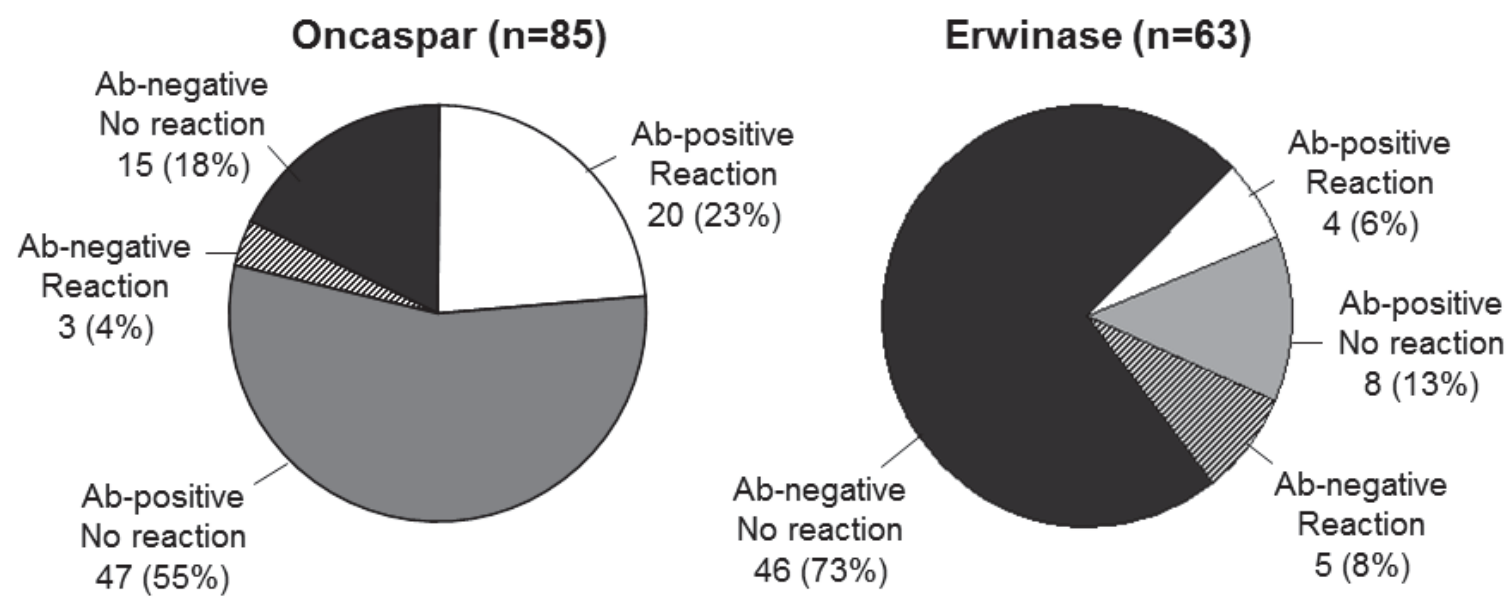

Figure 2-8. The frequency of hypersensitivity to Oncaspar and Erwinase

The proportion of patients exhibiting secondary allergic reactions and antibody positivity among the patients who had samples tested for anti-Oncaspar $(\mathrm{n}=85)$ or anti-Erwinase (n $=63$ ) after they received Oncaspar or Erwinase. Ab, antibody against Oncaspar (left) or Erwinase (right). 


\section{Antibodies and serum asparaginase activity}

In addition to testing the clinical utility of asparaginase antibodies as a tool to predict or confirm clinical allergy, antibodies have been hypothesized to be important as indicators of lower systemic exposure to asparaginase. Indeed, we found that in vivo serum asparaginase activity after Elspar was lower in the samples positive for antibodies than in those negative for antibodies $\left(P=7 \times 10^{-6}\right)$, and was inversely related to the level of antibody (based on OD readings, $P=7 \times 10^{-11}$; Figure 2-9).

To determine whether antibody was also inversely related to asparaginase activity after secondary treatment with Erwinase and Oncaspar, we measured in vivo serum asparaginase activity in 19 patients on Erwinase and 30 patients on Oncaspar at the time of the anti-Elspar antibody measurement. Asparaginase activity did not differ by antiElspar antibody positivity in patients who received second line treatment with Erwinase $(P=0.29)$, but it was lower (median $0.03 \mathrm{IU} / \mathrm{mL}$, range $0-2.73 \mathrm{IU} / \mathrm{mL}$ ) in those who were positive for anti-Elspar antibody and received second line treatment with Oncaspar compared to those who were antibody negative (median $2.04 \mathrm{IU} / \mathrm{mL}$, range 1.07-2.77 $\mathrm{IU} / \mathrm{mL} ; P=0.005)$.

The neutralizing effect of asparaginase antibodies was also confirmed by the ex vivo experiment (Figure 2-10). Patient sera that were positive for anti-Elspar antibodies neutralized up to about $60 \%$ of asparaginase activity of Elspar. There was a linear correlation between the level of antibody (based on OD readings) and the neutralizing capacity of patient samples (R-squared $=0.82, P=6.9 \times 10^{-21}$; Figure 2-10A). Similar neutralizing effect on Oncaspar activity was observed for patient sera with anti-Oncaspar antibodies, although not as strong as Elspar (R-squared $=0.28, P=3.4 \times 10^{-5}$; Figure 2-10B).

\section{Antibodies and adverse effects}

As we have shown that asparaginase can potentiate exposure to dexamethasone, ${ }^{30}$ we investigated whether cumulative asparaginase antibody titer affected the risk of symptomatic (grade 2 to 4 ) osteonecrosis. After adjustment for age and treatment arm, risk factors for the osteonecrosis, higher cumulative antibody titer (based on antibody AUC) was associated with lower risk of symptomatic osteonecrosis (odds ratio for $\log _{2}$ antibody $\mathrm{AUC}=0.83 ; 95 \% \mathrm{CI}, 0.78-0.89 ; P=0.007$; Table 2-7), consistent with antibodies causing less asparaginase exposure.

We also compared the cumulative risk of symptomatic osteonecrosis between patients negative for anti-Elspar antibody (i.e., anti-Elspar antibody AUC $\leq 2.12$ ) and those with detectable antibody (i.e., anti-Elspar antibody AUC > 2.12; Figure 2-11). There was a lower cumulative incidence of symptomatic osteonecrosis in those with a higher anti-Elspar antibody AUC who were older than 10 years of age (in the LR arm, $P$ $=0.048$; and the SHR arm, $P=0.037$ ), and in younger patients (in the LR arm, $P=$ 0.047; but not on the SHR arm, $P=1.0$; Figure 2-11). 

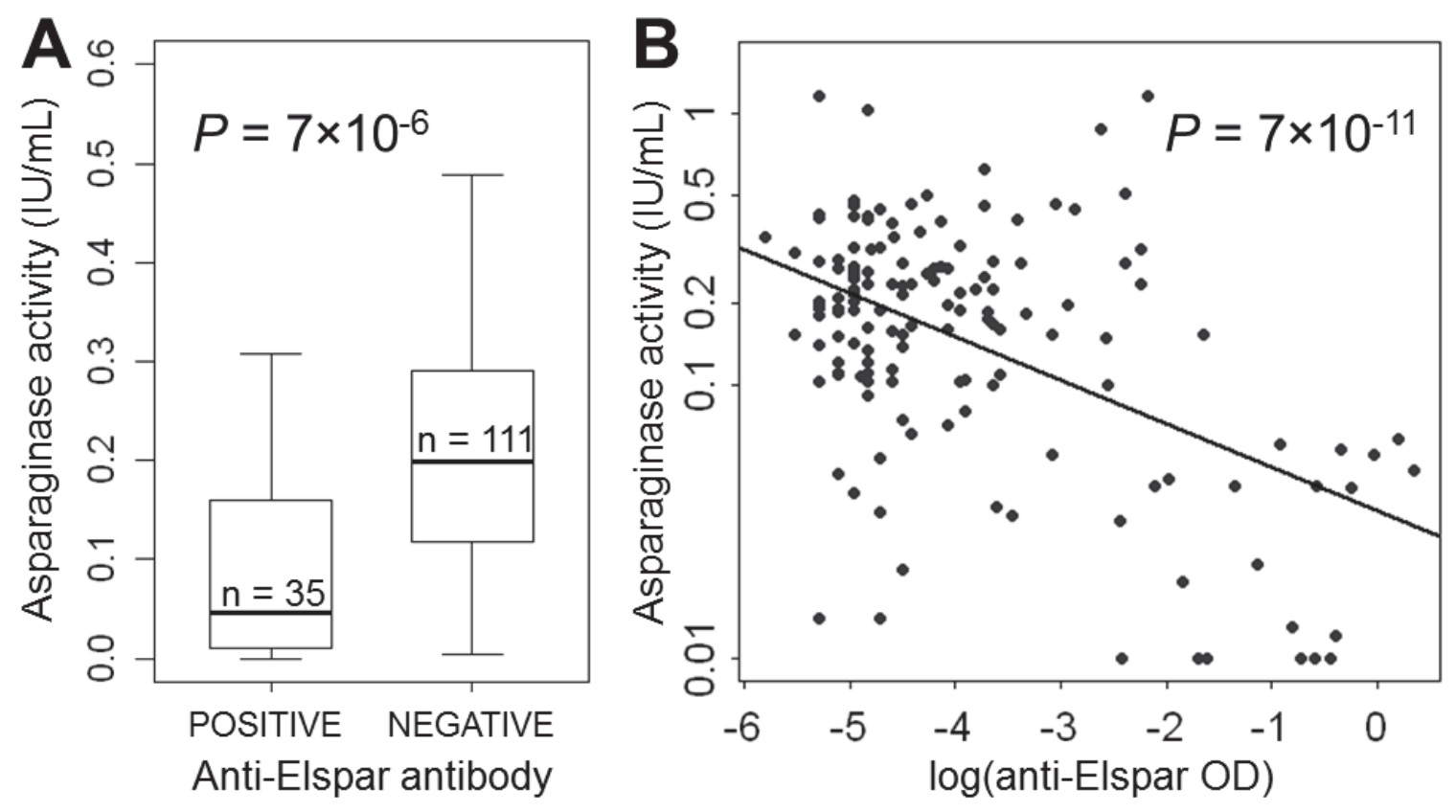

Figure 2-9. Correlation between serum asparaginase activity and antibody level Serum asparaginase activity (IU/mL) measured 6-8 days after the last Elspar dose of $25000 \mathrm{U} / \mathrm{m}^{2}$. (A) Asparaginase activity was significantly lower in the samples positive than in those negative for anti-Elspar antibodies. Box plots: median, quartiles, non-outlier range. (B) Asparaginase activity inversely correlated with anti-Elspar OD reading of serum samples. Individual data points and the regression line are shown for all cases $(\mathrm{n}=$ 146). 

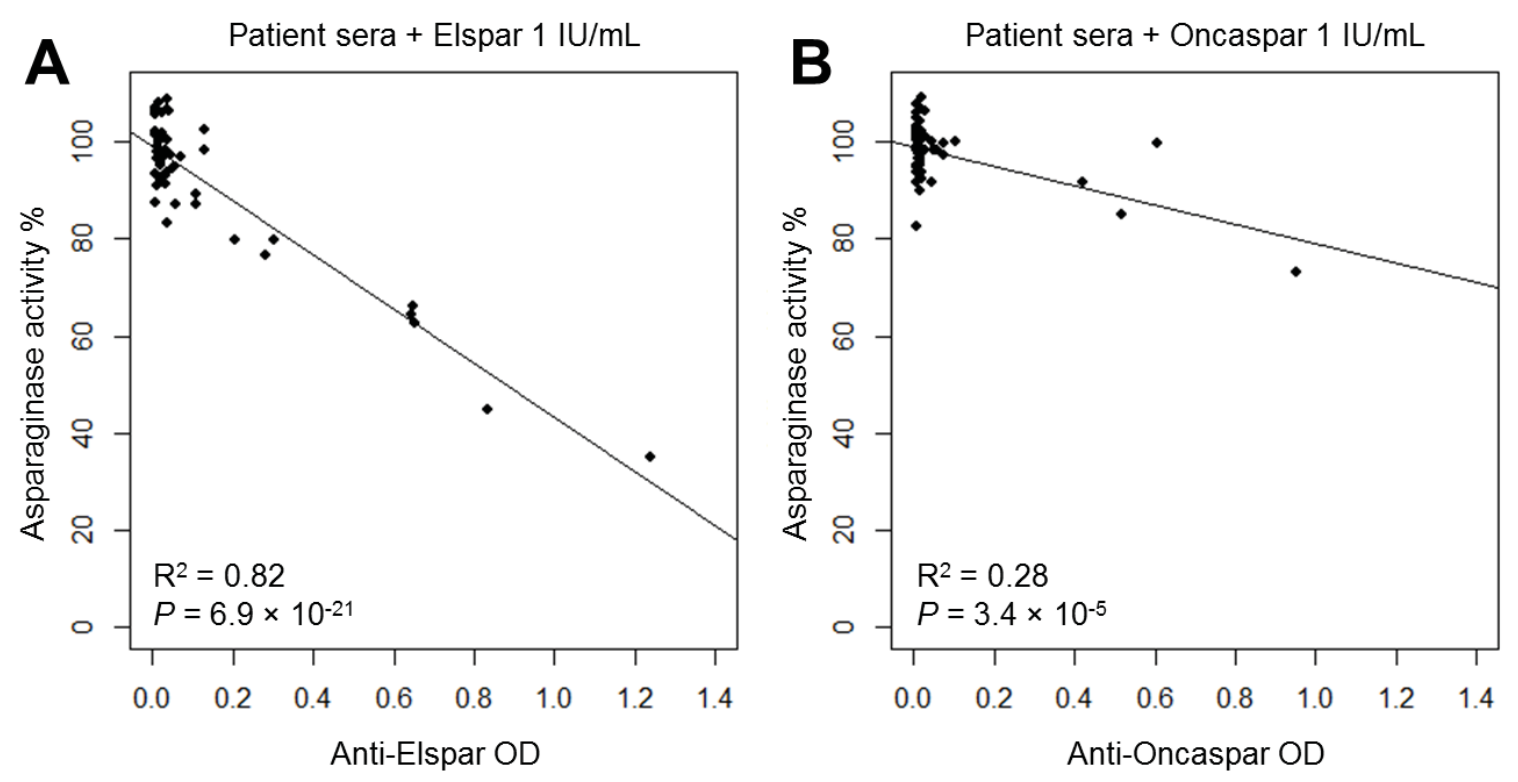

Figure 2-10. Ex vivo neutralization of patient sera with antibodies Patient serum samples were incubated with (A) Elspar (1.0 IU/mL) or (B) Oncaspar (1.0 $\mathrm{IU} / \mathrm{mL}$ ) for 1 hour. The remaining activity of the mixture was plotted against the antibody OD reading for each serum sample. Regression lines are shown.

Table 2-7. Multivariate analysis showed that low anti-Elspar antibody areaunder-curve (AUC) was a risk factor of osteonecrosis $(n=360)$

\begin{tabular}{lcc}
\hline Patient characteristics & $\boldsymbol{P}$ & Hazard ratio $\mathbf{( 9 5 \%}$ CI) \\
\hline Older than 10 years & $4.0 \times 10^{-7}$ & $5.52(2.84-10.71)$ \\
Standard/high-risk arm & 0.053 & $2.03(0.99-4.16)$ \\
Log2 (anti-Elspar antibody AUC) & 0.007 & $0.83(0.78-0.89)$ \\
\hline
\end{tabular}

Association with risk of osteonecrosis was assessed using Cox proportional hazard model. Log2(anti-Elspar antibody AUC) was treated as continuous variable; e.g. a hazard ratio of 0.83 suggests $83 \%$ risk of osteonecrosis for every two-fold increase in AUC. Other characteristic features are dichotomized for presence versus absence of the indicated feature. 
A

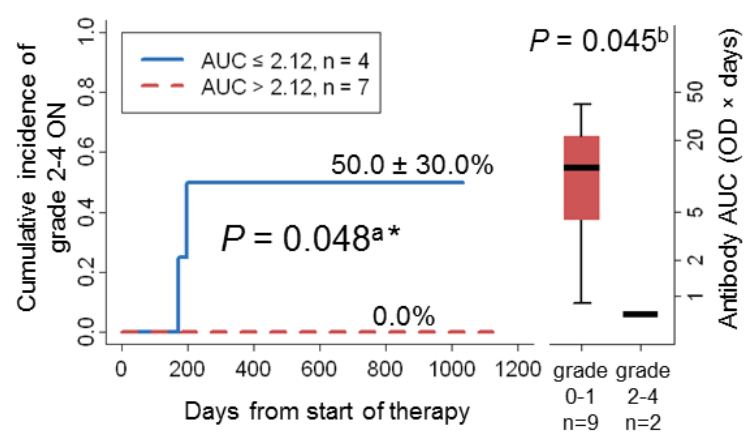

C

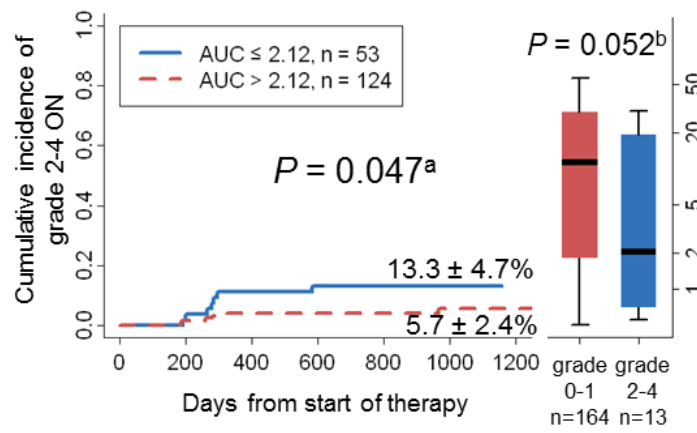

B Standard/high-risk (Age $\geq 10$ years)

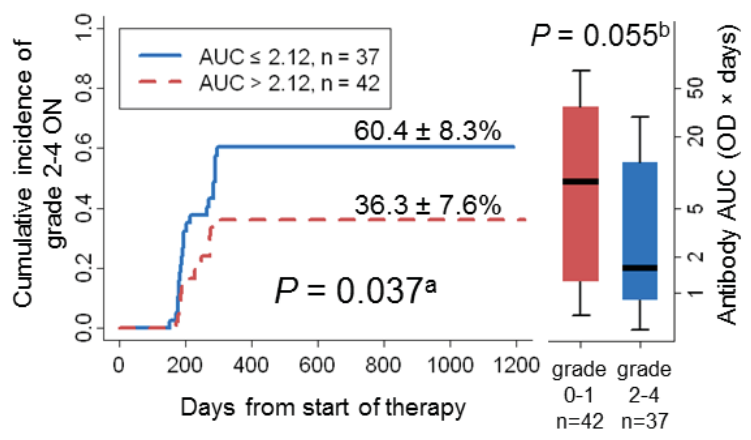

D Standard/high-risk (Age $<10$ years)

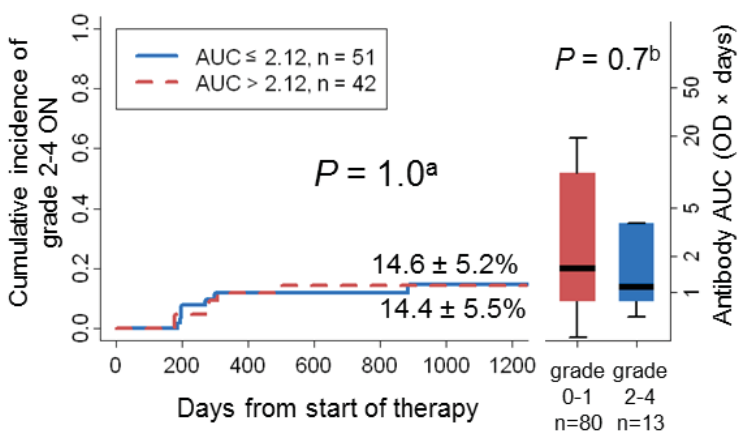

Figure 2-11. Cumulative incidence of osteonecrosis based on anti-asparaginase antibody AUC

Patients were stratified by low-risk (LR) versus standard/high-risk (SHR) treatment arm and age at diagnosis: (A) patients in the LR arm with age greater than 10 years; (B) patients in the SHR arm with age greater than 10 years; (C) patients in the LR arm with age under 10 years; (D) patients in the SHR arm with age under 10 years. Left:

Cumulative incidence of symptomatic (grade 2 to 4 ) osteonecrosis $(\mathrm{ON})$ in patients with anti-Elspar antibody AUC (OD $\times$ days) lower than 2.12 (blue) versus greater than 2.12 (red). Right: box plot of anti-Elspar antibody AUC in patients with (blue) versus without symptomatic $\mathrm{ON}$ (red). ${ }^{\mathrm{a}} P$ value based on log-rank test. ${ }^{\mathrm{b}} P$ value based on Wilcoxon rank sum test. *Fisher's exact test $(P=0.11)$ was also performed because the sample size is small. 
Seven patients had pancreatitis before the end of reinduction II, and five of them were positive for antibody. Twenty-eight patients had thrombotic events before the end of reinduction II, and seven of them were positive for antibody. After adjusting for age and treatment arm, there was no apparent association between antibody positivity and pancreatitis $(P=0.22)$ or thrombosis $(P=0.20)$.

\section{Discussion}

Although the relationship between antibodies and allergic reactions to asparaginase has been observed in many studies, ${ }^{32,33,37,117}$ this is the first report focusing on the diagnostic utility of asparaginase antibody measures to predict or to confirm clinical allergy to asparaginase. Particularly in children, differentiating allergy from other diagnoses can be challenging. In the context of an asparaginase-intensive front-line clinical trial and by dichotomizing samples as positive versus negative, the most informative sample (at week 7 of continuation phase) had reasonably high $(88 \%)$ sensitivity to predict reactions in the next 3 weeks but less favorable specificity (68\%) to confirm past reactions (Table 2-6). In this regard, there was a relatively frequent occurrence of silent hypersensitivity during therapy $(22 \%, 90$ of 410 patients on the protocol). Based on this test performance, for every 100 patients with a reaction during weeks 1-9, 88 would be expected to have positive and 12 would have negative antibody tests during this time period (at week 7); for every 100 patients without an allergy, 32 would have positive and 68 would have negative antibody tests at that time (week 7). The diagnostic value of antibody test at this time point is similar to those of some widely used clinical laboratory tests, such as the diagnosis of venous thromboembolism with d-dimer (sensitivity $80-95 \%$ and specificity $40-70 \%$ ); ${ }^{123}$ the prediction of rheumatoid arthritis with rheumatoid factor (sensitivity $40-80 \%$, specificity $70-90 \%$ ) $;{ }^{124}$ and the diagnosis of systemic lupus erythematosus with antinuclear antibody (sensitivity $93 \%$, specificity $50-80 \%)^{125}$

We were unable to identify a more informative time point that yielded better antibody test performance. The early antibody test at the end of induction lacked the sensitivity to predict future clinical allergy. The later antibody tests (i.e., week 17) did not confirm as well for patients in the LR arm; for those in the SHR arm, the week 17 result had slightly reduced sensitivity (72.7\%) and increased specificity (88.2\%), but clinically, it would not be possible to use such a result for making therapeutic decisions. Because antibody levels tend to increase over time with successive asparaginase exposures, even in the absence of a reaction, comparisons between reacting and non-reacting patients should control for place-in-therapy. In our study, serum samples were timed uniformly relative to therapy but were not available at the exact time of the reaction in most cases (and control samples, of course, would not have been available in non-reacting patients at exactly comparable times); thus, we were not able to assess the specificity of the serum anti-Elspar antibody test at the time of clinical reaction to Elspar. However, even if we limited analysis to those reacting patients who had serum obtained 5-30 days before or after the allergy ( $\mathrm{n}=132$ patients), the estimated sensitivity was not higher than $80 \%$ (Figure A-7). 
We also investigated whether the predictive utility of the antibody measures was improved by leaving the measure as a continuous measure (OD of anti-Elspar antibody titers) instead of dichotomizing the results as positive versus negative. We observed a linear correlation between the antibody OD at week 7 and the probability of patients having a clinical reaction in the subsequent 3-week asparaginase course, and a correlation between week 7 antibody OD and the proportion who had clinical reactions in the previous 6-week asparaginase course (Figure 2-7). For the LR patients with anti-Elspar OD $>0.39$ at week $7,90 \%$ of them are predicted to have an event in the subsequent course; for the SHR patients with anti-Elspar OD $>1.06$ at week 7, 80\% of them would have had an event in the previous course. However, falsely positive readings (high OD readings in patients with no known reaction) remained a limitation, and thus we were not able to define an alternative universal threshold for distinguishing positive from negative antibody status that resulted in better overall balanced test performance (based on the ROC curve of the antibody tests, see Figures 2-6 and A-5).

In addition to the possible utility of an anti-asparaginase antibody test to facilitate diagnosis of allergy, antibodies may be an indicator of reduced serum asparaginase exposure. In samples obtained at a uniform time (6-8 days) post-dose, we found that serum asparaginase activity was indeed inversely related to antibody level (Figure 2-9), consistent with reports from other groups..$^{32,40,42,43}$ The ex vivo experiment also showed that asparaginase activity was inhibited by adding patient sera with antibodies (Figure 2-10), suggesting that attenuation of serum asparaginase activity may be due to a direct neutralizing effect of antibodies. However, it is also possible that the presence of antibodies is an indicator of other immune-based mechanisms that enhance drug clearance (e.g., via the reticuloendothelial system). This may explain why a few samples had low asparaginase activity despite being low in asparaginase antibodies (Figure 2-9).

We explored whether the presence of antibodies, with its likely attendant reduced exposure to asparaginase over time, would reduce other adverse effects of asparaginase therapy. Even though dexamethasone is the major cause of osteonecrosis in children with ALL, ${ }^{89,96,126-128}$ we have shown that asparaginase can increase the risk of osteonecrosis, ${ }^{96}$ which we hypothesize could be because asparaginase inhibits protein synthesis, decreases dexamethasone clearance, ${ }^{30}$ alters lipid metabolism, ${ }^{129,130}$ and induces coagulopathy. ${ }^{131,132}$ Because osteonecrosis is a long-term complication, we used antibody AUC as a measurement of long-term exposure to antibodies against Elspar, the front-line asparaginase preparation. We observed that, among younger patients on the LR arm and all older patients on either LR or SHR arm, those with high antibody AUC were at lower risk to develop symptomatic osteonecrosis than those with low antibody AUC (Figure 2-11). The difference was not apparent among the younger patients on the SHR arm, possibly because younger patients are less prone to osteonecrosis, and those on the SHR arm also had lower antibodies, perhaps too low to have any effect on the development of osteonecrosis (Figure 2-11D, right panel). In multivariate analysis, after adjusting for age and treatment arm, antibody AUC was significantly associated with grade 2-4

osteonecrosis (Table 2-7). This is the first report that anti-asparaginase antibodies were inversely related to the risk of osteonecrosis, but is consistent with our hypothesis that asparaginase potentiates glucocorticoid effects. ${ }^{96}$ We recently reported that asparaginase 
antibodies were associated with a higher risk of central nervous system relapse, ${ }^{21}$ but did not find that asparaginase antibodies were related to pancreatitis or thrombosis, perhaps indicating that these latter two adverse events are less "dose-related" than others.

In the Total XV study, with Elspar as the front-line asparaginase preparation, the frequency of clinical hypersensitivity to Elspar was 41\% (169/410) for all patients who started with Elspar, and the incidence of IgG antibodies to Elspar was 58\% (236/410), similar to the average from other reported series..$^{31,40,43,117}$ The hypersensitivity appeared to differ by treatment arm and immunophenotype, consistent with the result from our previous ALL trial, Total XIII. ${ }^{31,117}$ Patients on the LR arm exhibited more hypersensitivity to asparaginase than did those on the SHR arm, possibly because the LR arm included less immunosuppressive chemotherapy and included longer periods of asparaginase "holiday" followed by re-challenge, a practice noted to be associated with allergy by others. ${ }^{116}$

There was a very high frequency of "silent hypersensitivity" observed in patients who subsequently received Oncaspar (55\% of patients with no allergy had positive antibodies against Oncaspar). However, 24\% of patients who never received Oncaspar had positive antibodies at week 7 and 17, which could be due to cross reactivity between sera positive to both Elspar and Oncaspar in patients who are exposed initially to Elspar (Oncaspar is pegylated Elspar). ${ }^{133}$ Consistent with that idea, only $13 \%$ of patients who received Erwinase had silent hypersensitivity, compared to $6 \%$ of patients who never received Erwinase. Allergy was also more common $(P=0.05)$ in those who received Oncaspar rather than Erwinase as their second-line agent, and serum asparaginase activity was lower in those with (compared to those without) anti-Elspar antibodies who received Oncaspar $(P=0.005)$, but not in those who received Erwinase $(P=0.29)$, consistent with recent reports. ${ }^{42}$ Together, these data suggest that Erwinase may be preferred over Oncaspar in those who receive primary treatment with Elspar and experience allergy, although it is possible that such a decision depends upon the dose of Oncaspar versus Erwinase and the absolute level of anti-Elspar antibodies. ${ }^{42}$

Interestingly, patients with T-cell ALL were less likely to develop asparaginase allergy and had lower antibody levels at post-asparaginase time points than those with Blineage ALL. The mechanisms underlying this difference are unclear, but suggest an intriguing added value for asparaginase in T-cell ALL.

\section{Conclusion}

In summary, we comprehensively analyzed anti-Elspar antibody in an asparaginase-intensive front-line clinical trial. In this context, in which Elspar is given as the primary form of asparaginase, serum anti-Elspar antibodies have good utility as tools to help make the diagnosis of clinical hypersensitivity. Our data show that higher antibody levels are associated with greater clinical pharmacologic effects including a higher risk of clinical allergy, and a greater attenuation of serum asparaginase enzyme 
activity with associated lower risk of osteonecrosis. Thus, measures of serum antibodies to asparaginase can be useful in patients with ALL. 


\section{CHAPTER 3. POTENTIATING EFFECT OF ASPARAGINASE ON GLUCOCORTICOID-INDUCED OSTEONECROSIS}

\section{Introduction}

Osteonecrosis is a common complication in patients treated for ALL, primarily due to the use of glucocorticoids (e.g. dexamethasone and prednisone). ${ }^{88-92}$ Proposed mechanisms of glucocorticoid-induced osteonecrosis included inhibition of angiogenesis, bone marrow adipogenesis, hypercoagulation, and apoptosis of endothelial cells and osteocytes. ${ }^{93}$ Our study recently suggested that damage to blood vessels supplying bone (arteriopathy) plays a primary role in the pathogenesis of osteonecrosis. ${ }^{94}$ Clinical studies have identified several risk factors for osteonecrosis in ALL patients, including adolescent age, ${ }^{88-92,126,128,134-136}$ female sex, ${ }^{88,89,91,134,136}$ white race, ${ }^{89,126}$ and exposure to intensive, long-term glucocorticoids. ${ }^{88-92}$

The reported frequency of osteonecrosis has varied widely from $1 \%$ to $20 \%$ among different ALL protocols ${ }^{88-92,126,128,134-136}$ even with relatively similar glucocorticoid regimens, and interactions with accompanying antileukemic medication is suspected to contribute to this variability. Evidence is accumulating that asparaginase can increase the risk of osteonecrosis. Asparaginase exposure was associated with decreased clearance and increased exposure of dexamethasone, ${ }^{30}$ and the development of an antibody response against asparaginase is associated with a decreased 2incidence of symptomatic osteonecrosis. ${ }^{87}$ A recent ALL trial showed that extra doses of asparaginase during interim maintenance therapy caused more osteonecrosis in patients receiving prednisone. ${ }^{95}$ Moreover, the hypoalbuminemia caused by asparaginase was also associated with osteonecrosis risk. ${ }^{90}$

However, these clinical data are not definitive, in that they do not come from a trial in which patients receive identical glucocorticoid regimens with different exposures to asparaginase. Thus, our study aimed to address the impact of asparaginase on osteonecrosis in a controlled, pre-clinical model. We also evaluated the impact of host factors (e.g. age and gender), treatment factors (e.g. glucocorticoid regimen and duration), and environmental factors (mice derived from in-house breeding colonies vs. shipped from vendor) on dexamethasone tolerance and the development of osteonecrosis using this mouse model.

\section{Methods}

\section{Chemicals}

Dexamethasone sodium phosphate solution was purchased from American Pharmaceutical Partners, Inc. (Schaumburg, IL). PEG-asparaginase (Oncaspar) was a gift from Sigma Tau (Gaithersburg, MD). Tetracycline was purchased from Sigma-Aldrich 
(St. Louis, MO), and sulfamethoxazole/trimethoprim oral suspension was obtained from Hi-Tech Pharmacal Co., Inc. (Amityville, NY).

\begin{abstract}
Animals
Unless otherwise specified, we studied male BALB/cJ mice (age 24 or 28 days) bred in our own facilities (SJCRH, Memphis, TN; the original breeding pairs were from Jackson Laboratories). We also studied BALB/cJ mice directly shipped from Jackson Laboratories (Bar Harbor, ME), or male BALB/cAnNHsd mice (age 24 or 28 days) obtained from Harlan Laboratories (Houston, TX). The details are described in Appendix B (Table B-1 and Figure B-1).
\end{abstract}

\title{
Asparaginase and dexamethasone pharmacokinetics
}

To determine the effect of asparaginase treatment on dexamethasone pharmacokinetics, we conducted an experiment using males at 6-8 weeks of age. Mice received dexamethasone at $4 \mathrm{mg} / \mathrm{L}$ (drinking water) and PEG-asparaginase intraperitoneally (i.p.) at $1500 \mathrm{IU} / \mathrm{kg}$ for 0 to 4 doses at an interval of 3.5 days (Figure 3-1). All mice were sacrificed at the end of week-2 (3.5 days after last asparaginase dose). Blood was collected via cardiac puncture with anticoagulant, and plasma was measured for the levels of dexamethasone, asparaginase, amylase, lipase, albumin, triglyceride and cortisol (Figure 3-2 and Appendix B).

\section{Mouse model of dexamethasone-induced osteonecrosis}

A series of experiments were conducted to refine a mouse model of osteonecrosis (see Appendix B Tables B-1 and B-2, and Figures B-1 through B-7). From postnatal day 28 (P28) or day 24 (P24), male and female BALB/cJ and BALB/cAnN mice were treated with 4 or $8 \mathrm{mg} / \mathrm{L}$ dexamethasone in drinking water, equivalent to 1.33 or 2.66 $\mathrm{mg} / \mathrm{kg} /$ day, assuming that each mouse weighed $15 \mathrm{~g}$ and consumed $5 \mathrm{~mL}$ water daily. ${ }^{137}$ Mice in dexamethasone groups were treated with dexamethasone with antibiotics, except in a pilot experiment where mice were given dexamethasone without antibiotics. The control groups were treated with only antibiotics. Dexamethasone was given at 4 or 8 $\mathrm{mg} / \mathrm{L}$ in drinking water, equivalent to 1.33 or $2.66 \mathrm{mg} / \mathrm{kg} /$ day, assuming that each mouse weighed $15 \mathrm{~g}$ and consumed $5 \mathrm{~mL}$ water daily. ${ }^{137}$ Treatment generally lasted for 6 weeks, while a subset of BALB/cAnN was treated for up to 8 weeks in an effort to increase the frequency of osteonecrosis. Antibiotic prophylaxis to prevent dexamethasone-induced infections consisted of tetracycline $(1 \mathrm{~g} / \mathrm{L})$ constantly, and sulfamethoxazole $(600 \mathrm{mg} / \mathrm{L})$ and trimethoprim $(120 \mathrm{mg} / \mathrm{L})$ given 3.5 days per week. These antibiotics have no effect on osteonecrosis. ${ }^{96}$ Water bottles were changed every 3.5 days. 


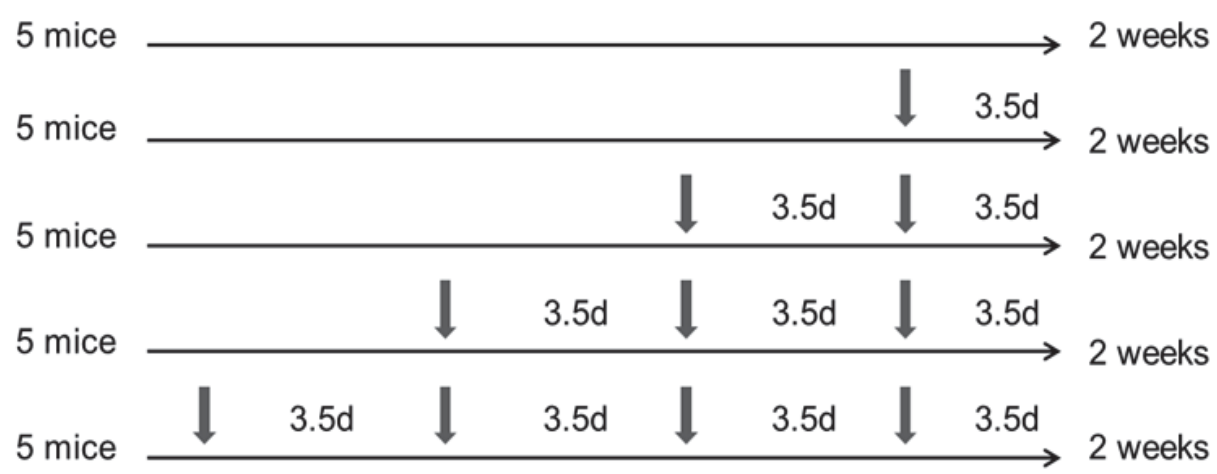

$4 \mathrm{mg} / \mathrm{L}$

$4 \mathrm{mg} / \mathrm{L} \quad 1500 \mathrm{IU} / \mathrm{kg}$ (1)

$4 \mathrm{mg} / \mathrm{L} \quad 1500 \mathrm{IU} / \mathrm{kg}(2)$

$4 \mathrm{mg} / \mathrm{L} \quad 1500 \mathrm{IU} / \mathrm{kg}(3)$

$4 \mathrm{mg} / \mathrm{L} \quad 1500 \mathrm{IU} / \mathrm{kg}(4)$

ON experiment

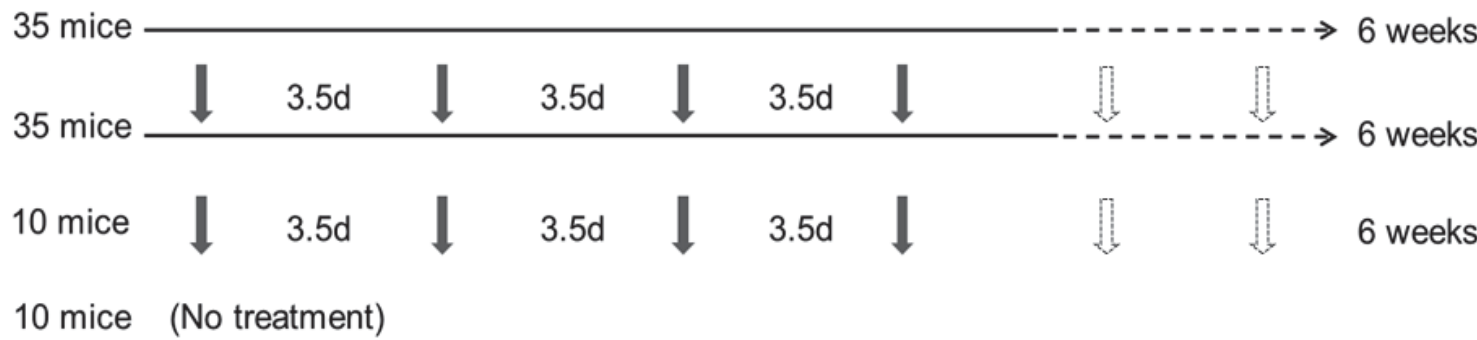

$2 \mathrm{mg} / \mathrm{L}$

$2 \mathrm{mg} / \mathrm{L} \quad 1200 \mathrm{IU} / \mathrm{kg}(12)$

$1200 \mathrm{IU} / \mathrm{kg}(12)$

10 mice (No treatment)

\section{Figure 3-1. Dexamethasone and asparaginase treatment regimens}

Horizontal arrows represent dexamethasone treatment $(2$ or $4 \mathrm{mg} / \mathrm{L}$ in drinking water) and vertical arrows represent PEGasparaginase (Oncaspar) treatment (1200 or $1500 \mathrm{IU} / \mathrm{kg}$ twice weekly via i.p. injection). Dashed lines and arrows indicate continuous treatment after week 2. The dose of dexamethasone (in drinking water), and the dose and number of i.p. injections of asparaginase are shown on the right. PK, pharmacokinetics. ON, osteonecrosis. DEX, dexamethasone. ASP, PEGasparaginase (Oncaspar). 


\section{Effect of asparaginase on osteonecrosis}

Male P24 BALB/cJ in-house bred mice were used to determine the effect of asparaginase on dexamethasone-induced osteonecrosis. Ninety mice were divided into four groups: 35 mice were assigned to the dexamethasone-alone group, 35 mice to the dexamethasone and asparaginase group, 10 mice to the asparaginase-alone group and 10 mice to untreated control (Figure 3-1). Dexamethasone was given at $2 \mathrm{mg} / \mathrm{L}$ (equivalent to $0.67 \mathrm{mg} / \mathrm{kg} /$ day) in drinking water for 6 weeks, and asparaginase was injected i.p. at $1200 \mathrm{IU} / \mathrm{kg}$ twice weekly. The same antibiotic prophylaxis was used as described above.

\section{Plasma dexamethasone concentration and asparaginase activity}

At the time of sacrifice (between 9am and noon), mice were anesthetized with $2 \%$ isoflurane, and blood was collected via cardiac puncture. Plasma was frozen at $-80^{\circ} \mathrm{C}$ until assayed. Dexamethasone concentration was quantified by high-performance liquid chromatography (HPLC). Plasma asparaginase activity was determined using a highthroughput assay by monitoring the enzymatically-coupled oxidation of reduced nicotinamide adenine dinucleotide $(\mathrm{NADH})$ to $\mathrm{NAD}(+) .{ }^{138}$ The linear range of the assay was established from 0.025 to $2.2 \mathrm{IU} / \mathrm{mL}$; if the activity was out of the range, the plasma was diluted with serum albumin to be in range. Please see Appendix $\mathbf{B}$ for the detailed assay protocols.

\section{Histological evaluation of osteonecrosis and arteriopathy}

Osteonecrosis was determined by empty lacunae, pyknotic nuclei or ghost nuclei in osteocytes in the bone trabeculae, and necrosis of the adjacent marrow and stromal elements, as described previously. ${ }^{94,96,137}$ Arteriopathy was defined by the presence or absence of lesions in arteriolar branches of the medial genicular artery located along the surface of the distal femoral condyles. ${ }^{94}$ If there was no evaluable arteriolar branch present, the case was categorized as "unknown". Mice with osteonecrosis and/or arteriopathy in one or both legs were classified as positive for osteonecrosis and/or arteriopathy.

\section{Statistical analysis}

The Chi-square test was used to evaluate intergroup differences in categorical variables. The Mann-Whitney test was used to compare continuous variables. A P-value of less than 0.05 was considered statistically significant. 


\section{Results}

\section{Plasma dexamethasone concentration was increased by asparaginase treatment}

After 2 weeks of treatment with dexamethasone at $4 \mathrm{mg} / \mathrm{L}$ and PEG-asparaginase at $1500 \mathrm{IU} / \mathrm{kg}$ for 0-4 doses, all 25 mice in the PK experiment were evaluable at the end of week 2 ("pharmacokenetics experiment"; Figure 3-1). There was no significant difference in plasma asparaginase activity among the three groups $(15.3 \pm 6.5,14.5 \pm 7.7$ and $13.2 \pm 4.1 \mathrm{IU} / \mathrm{mL}$, respectively) that received 2,3 or 4 doses of asparaginase at 1500 IU/kg i.p. every 3.5 days. Those who received only one dose of asparaginase had slightly lower asparaginase activity $(11.9 \pm 2.6 \mathrm{IU} / \mathrm{mL}, P=0.02)$ compared with other groups. We observed a positive association between plasma dexamethasone concentration and asparaginase activity $(P=0.0001$; Figure 3-2). No mice developed osteonecrosis after only two weeks of treatment.

\section{Asparaginase treatment potentiated osteonecrosis and arteriopathy in dexamethasone-treated mice}

Based on the result of preliminary experiments, we studied the effect of asparaginase on dexamethasone-induced osteonecrosis using 24-day-old BALB/cJ male mice ("osteonecrosis experiment"; Figure 3-1). The mean weights of mice treated with asparaginase (14.7 $\pm 1.9 \mathrm{~g}$ with dexamethasone and $19.4 \pm 2.5 \mathrm{~g}$ without dexamethasone) were significantly lower than those who did not receive asparaginase $(17.4 \pm 1.5 \mathrm{~g}$ with dexamethasone and $26.8 \pm 2.3 \mathrm{~g}$ without dexamethasone; $P<0.0001)$ after 6 weeks of treatment. Thirty of $35(86 \%)$ mice in the dexamethasone-alone group and 27 of $35(77 \%$; $P=0.8)$ mice in the dexamethasone plus asparaginase group survived the 6 -week treatment. There were no deaths among the 10 untreated controls and the 10 asparaginase-alone mice.

Osteonecrosis developed in 12 of 27 (44.4\%) mice who received dexamethasone and asparaginase, significantly higher $(P=0.006)$ than in mice who received dexamethasone alone (3 of 30 or 10.0\%; Figure 3-3). An arteriolar branch coursing along the dorsal surface of the distal femur was detected in 47 of the 57 mice evaluated for osteonecrosis. Lesions were present in the vessels of 14 of $24(58 \%)$ mice who received both dexamethasone and asparaginase, compared with 4 of $23(17 \%)$ mice who received dexamethasone alone $(P=0.007)$. No mice in the control group or the asparaginase alone group developed either osteonecrosis or arteriopathy.

Consistent with the pharmacokinetic experiment, we observed an association between plasma dexamethasone concentration and asparaginase activity $(P=0.005$; see Appendix B, Figure B-8) in the osteonecrosis experiment. In the 27 mice that received both dexamethasone and PEG-asparaginase, there was a significant trend towards higher dexamethasone levels $(P=0.027)$ and higher asparaginase activity $(P=0.036$; Figure 3-4) in osteonecrosis-positive cases. A multivariate analysis suggested that plasma 


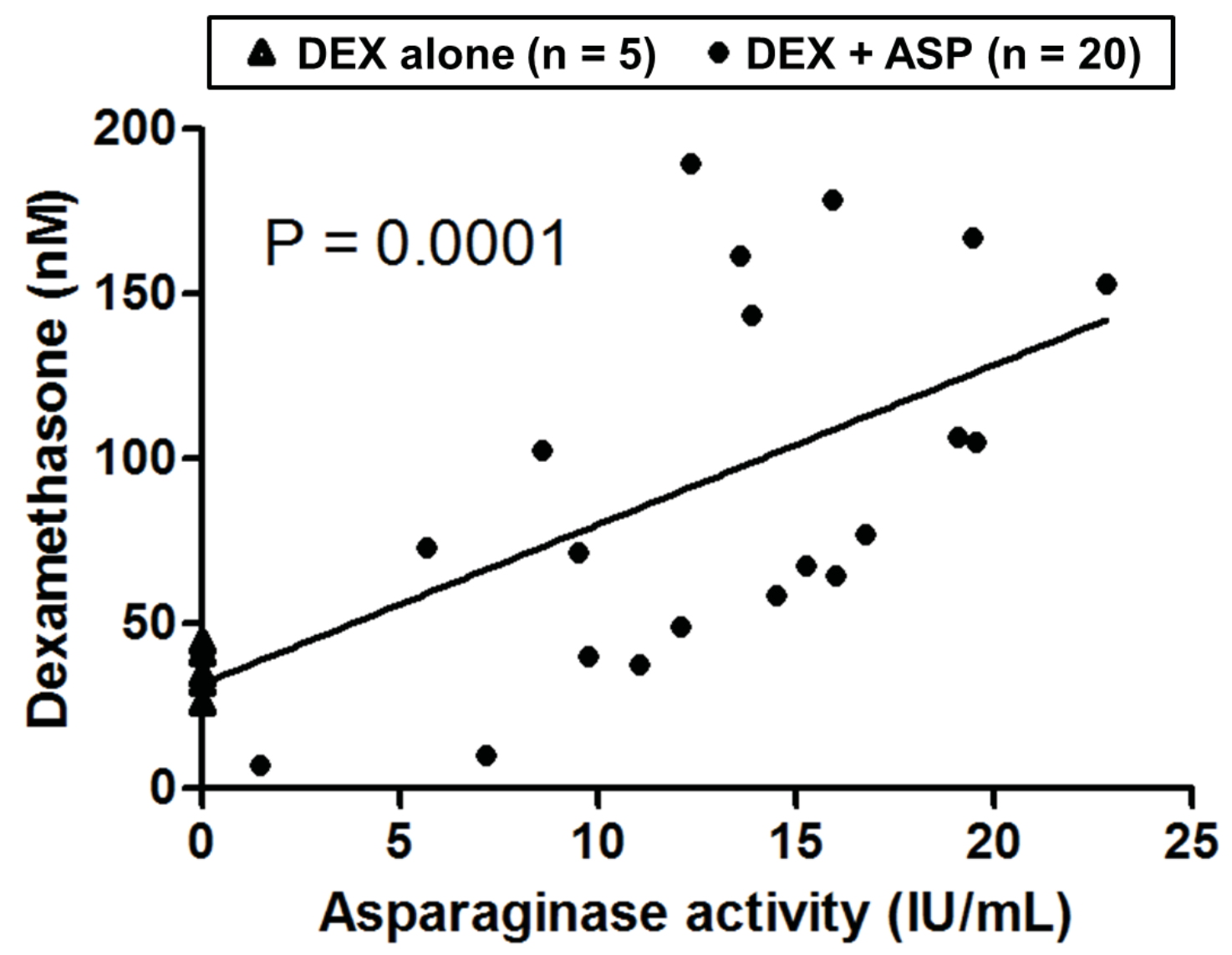

Figure 3-2. Plasma dexamethasone concentration was positively associated with asparaginase activity in PK experiment

Mice received dexamethasone (DEX; $4 \mathrm{mg} / \mathrm{L}$ in drinking water) for 2 weeks and 0-4 doses of PEG-asparaginase (ASP; 1500 IU/kg i.p.) at 3.5 day intervals. Samples Blood samples from dexamethasone-alone mice (triangles) and those received additional asparaginase (points) were collected at the end of week 2 (3.5 days after the last asparaginase injection). Each dot represents a mouse. Linear regression line is shown. 


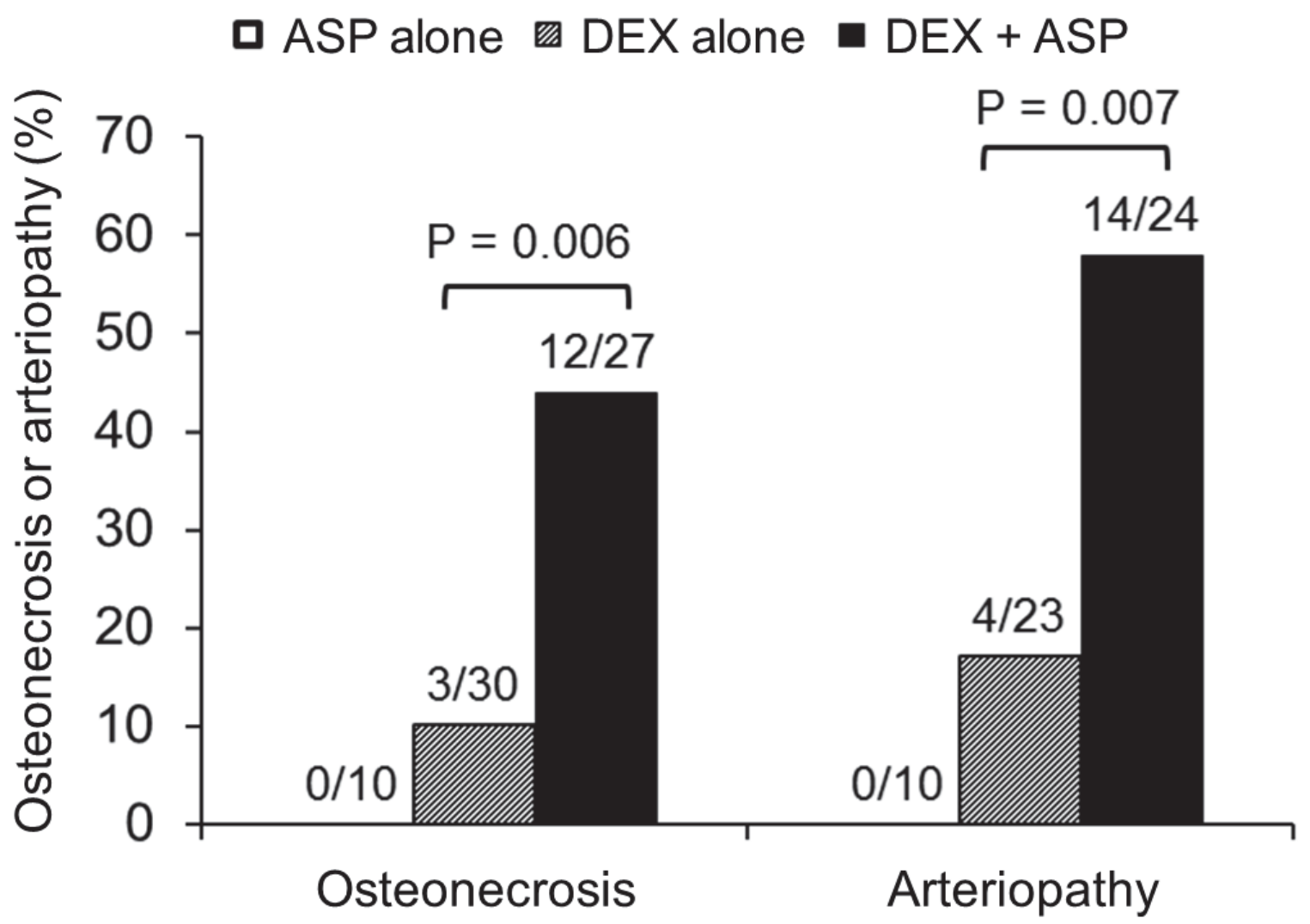

Figure 3-3. Asparaginase treatment potentiated osteonecrosis and arteriopathy in dexamethasone-treated mice

Chi-square $\mathrm{P}$ values were calculated between dexamethasone (DEX)-treated mice that received and vs. those did not receive asparaginase (ASP). 

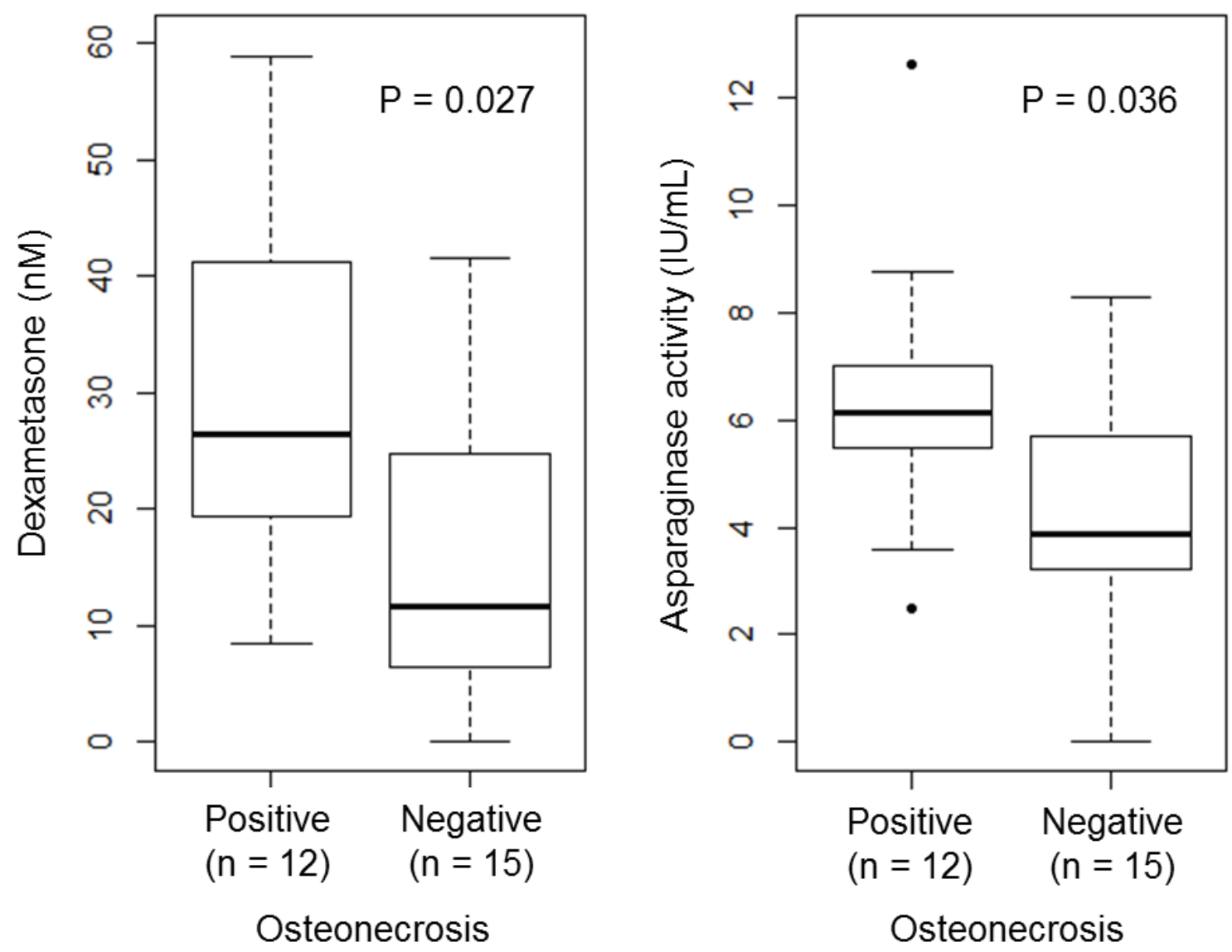

Figure 3-4. Osteonecrosis was associated with higher plasma dexamethasone and asparaginase levels in mice receiving both dexamethasone and asparaginase treatment

$\mathrm{BALB} / \mathrm{cJ}$ males received dexamethasone (4 mg/L) and PEG-asparaginase (1200 IU/kg i.p. twice weekly) for 6 weeks. Blood samples were collected approximately 3.5 days after last injection. 
dexamethasone $(\mathrm{OR}=1.01, P=0.029)$ and asparaginase concentrations $(\mathrm{OR}=1.06, P=$ 0.002 ) were both independently associated with osteonecrosis.

\section{Arteriopathy was likely the initiating event of osteonecrosis}

Among the 15 mice positive for osteonecrosis, one did not have an evaluable vessel present in the plane of section, and all the other $14(100 \%)$ were positive for arteriopathy. Among the 33 mice with no signs of osteonecrosis, arteriopathy was present in 4 mice $\left(12 \% ; P=9 \times 10^{-9}\right)$, consistent with our previous study which indicated that vascular damage may be the initiating event of osteonecrosis development. ${ }^{94}$

The histopathological changes of bone and vessels during dexamethasone and asparaginase treatment are shown in Figure 3-5. When arteriopathy was present without osteonecrosis (Figure 3-5D), it was evident in branches of the medial genicular artery supplying the distal femoral epiphysis (Figure 3-5E) with signs of luminal occlusion and loss of endothelium and smooth muscle cells; the interruption of blood supply was localized, resulting in reduced hematopoietic cells in bone marrow (Figure 3-5F). When arteriopathy and osteonecrosis were both present, long-term poor circulation led to death of osteocytes and necrosis of marrow and hematopoietic cells (Figure 3-5I) and ultimately, extensive necrosis of the bone (Figure 3-5G).

\section{Discussion}

Clinical studies have indicated interaction between asparaginase and glucocorticoids in pediatric patients receiving the two critical agents of ALL chemotherapy. ${ }^{21,30}$ Herein, we used a mouse model to confirm the clinical observations that systemic exposure of glucocorticoids was increased by asparaginase treatment, and we showed definitively that asparaginase treatment contributes to the osteonecrotic effect of glucocorticoids.

In the present study, we confirmed the positive association between plasma dexamethasone level and asparaginase activity in two independent experiments (Figure 3-2 and Figure B-8). Mice that received asparaginase and dexamethasone (2 - $4 \mathrm{mg} / \mathrm{L}$ in drinking water) had a higher level of plasma dexamethasone than those who received dexamethasone alone $(P=0.001$ and $P=0.005$ in two independent experiments respectively). Osteonecrosis was associated with higher systemic exposure to dexamethasone and asparaginase in the univariate $(P=0.027$ and $P=0.036$ respectively; Figure 3-4) and the multivariate analysis $(P=0.029$ and $P=0.002$, respectively).

Concurrent use of asparaginase and glucocorticoids can potentiate each other's effects. Asparaginase is associated with decreased clearance of dexamethasone, ${ }^{30}$ which could be due to its hypoproteinemic effects, possibly decreasing hepatic CYP3A or transporters. Also, the immunosuppressive effects of glucocorticoids also inhibit the antibody response against asparaginase and prevent its neutralizing effect, which in turn 


\section{Figure 3-5. Histology of osteonecrosis and arteriopathy}

$\mathrm{H} \& \mathrm{E}$ staining of representative stifle joints from mice negative for osteonecrosis (ON) and arteriopathy (Art; top panel), negative for osteonecrosis but positive for arteriopathy (middle panel), and positive for both osteonecrosis and arteriopathy (bottom panel). All mice received dexamethasone and PEG-asparaginase for 6 weeks. (A) Normal arteriole (arrow), marrow and trabecular bone. (B) Magnified cross-section of the arteriole in A. The inset shows the normal endothelial cells (dashed arrows) with elongated nuclei and smooth muscle cells (solid arrows) with round nuclei. (C) Magnification of boxed area in A, showing healthy osteocytes in lacunae (arrows) and healthy hematopoietic cells in marrow (asterisk). (D) Vessel with arteriopathy (arrow) and healthy bone with slightly decreased hematopoietic cells (asterisk). (E) Magnification of the arteriole in D, showing thickened, occluded blood vessel (arrow) and loss of endothelium and smooth muscle cells. (F) Magnification of boxed area in D, showing healthy osteocytes in lacunae (arrows) and decreased hematopoietic cells (asterisk). (G) Vessel with arteriopathy (arrow) and necrotic marrow and trabecular bone. (H) Magnification of the arteriole in G, showing thickened, occluded blood vessel (arrow) and loss of endothelium and smooth muscle cells. (I) Magnification of boxed area in G, showing empty lacunae and dead osteocytes (arrows) and necrotic marrow (asterisk). Scale bars are 500 microns in the left panel and 50 microns in the middle and right panels. 

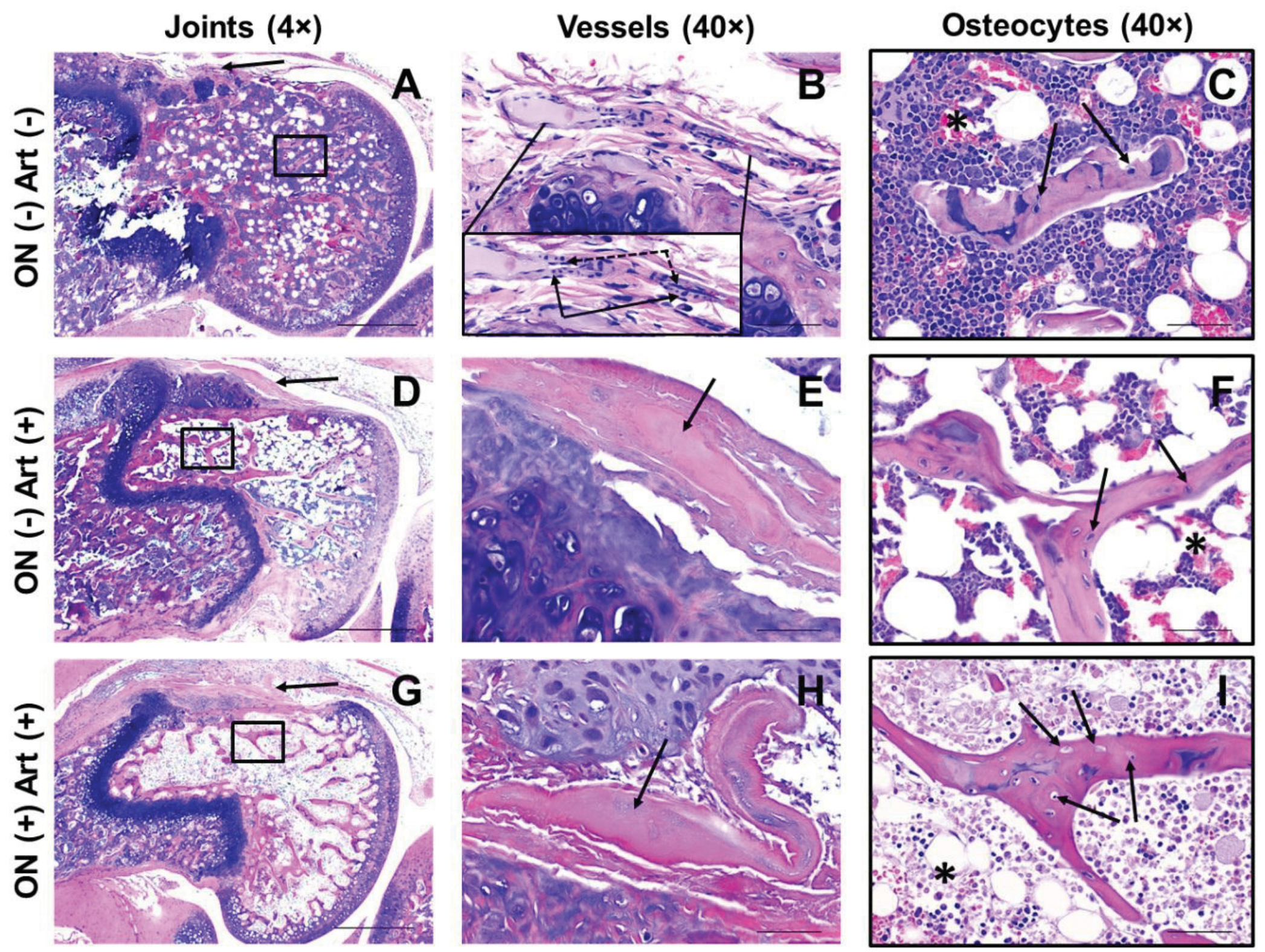
results in higher plasma asparaginase activity. ${ }^{87}$ In a front-line ALL study, St. Jude Total $\mathrm{XV}$, patients with antibodies against asparaginase had a lower risk of developing osteonecrosis than those who did not develop antibodies. ${ }^{87}$

Asparaginase is always used in therapy which includes glucocorticoids. Hanada et $\mathrm{al}^{131}$ reported a pediatric ALL patient who developed osteonecrosis during asparaginase therapy, but the patient had also received prednisone. In the present study, we did not observe any significant change in bone or vessels of mice receiving asparaginase alone, consistent with asparaginase enhancing the osteonecrotic effect of glucocorticoids, rather than a direct impact on osteonecrosis.

There are several possible mechanisms for the potentiating effect of asparaginase on glucocorticoid-induced osteonecrosis. Both asparaginase and glucocorticoids have been shown to induce a hypercoagulable state by suppression of anticoagulant factors such as antithrombin, plasminogen and d-dimer, and by elevation in F VIII/vWF complex. ${ }^{139}$ The hypercoagulable state may lead to impaired circulation, vascular damage and subsequent osteonecrosis. ${ }^{131,132}$ Interestingly, patients between 11 and 16 years had more significant alteration of anticoagulant and fibrinolytic parameters than children of other ages, ${ }^{140}$ consistent with the high susceptibility of adolescents to osteonecrosis. Moreover, alterations in lipid metabolism after asparaginase treatment ${ }^{129,130}$ may lead to formation of lipidic droplets that can be entrapped in the arterial lumen, followed by reduced blood flow and damage to the vascular endothelium. Asparaginase treatment is also associated with venous stasis and deep vein thrombosis in clinic ${ }^{141}$ and in animal models, ${ }^{142}$ although we did not observe such effects with asparaginase alone.

We previously showed preliminary data that native asparaginase could potentiate glucocorticoid-induced osteonecrosis. ${ }^{96}$ However, native E.coli asparaginase is no longer commercially available; it has been replace by PEG-asparaginase. In most ALL protocols, PEG-asparaginase is administered intravenously (i.v.) or intramuscularly (i.m.), and the typical doses range from $1000 \mathrm{IU} / \mathrm{m}^{2}$ to $3500 \mathrm{IU} / \mathrm{m}^{2}{ }^{2}{ }^{138,143-145}$ Plasma asparaginase activity at 3 days after receiving a usual recommend dose of PEG-asparaginase (2500 $\mathrm{IU} / \mathrm{m}^{2}$ i.v.) ranged from 2 to $4 \mathrm{IU} / \mathrm{mL}$ in patients. ${ }^{146}$ Herein, we used doses of PEGasparaginase (1200-1500 IU/kg i.p.) that have previously been shown to result in antileukemic effects in murine models. ${ }^{147}$ After injection of PEG-asparaginase at 1200 $\mathrm{IU} / \mathrm{kg}$ to mice, we achieved plasma asparaginase activity that was slightly higher $(5.4 \pm$ $2.9 \mathrm{IU} / \mathrm{mL})$ than those in patients receiving standard dose $\left(2500 \mathrm{IU} / \mathrm{m}^{2}\right)$, and may be more comparable to patients on high-dose asparaginase therapies.

Despite the anatomical and genetic differences between mouse and human, this mouse model yielded similar lesions in bone and arteries as those that have been reported clinically. Biopsy specimens of the femoral head from patients with early-stage osteonecrosis showed structural damage to arteriolar walls before necrosis of trabecular bone and marrow. ${ }^{148}$

We tested multiple host-related and treatment-related risk factors for osteonecrosis using this murine model (please see Appendix B for details). Osteonecrosis 
was associated with male sex, earlier start of treatment (before the onset of puberty), and higher dose and longer treatment duration with dexamethasone. All of these predisposing factors have been reported in clinical studies; ${ }^{89-91,126,128,134,136}$ however, in clinical studies that do show a gender difference, females are at higher risk than males. ${ }^{88,89,91,134,136}$ Why male mice have a higher risk than females is not clear. Among the mice of different substrains, sources and ages, four-week-old in-house bred BALB/cJ male mice displayed high susceptibility to osteonecrosis with an acceptable survival rate; therefore they may serve as a reliable and reproducible mouse model for studying glucocorticoid-induced osteonecrosis in the future.

In the pharmacokinetics experiment, we also measured plasma level of proteins and lipids after dexamethasone ( $4 \mathrm{mg} / \mathrm{L}$ for 2 weeks) and asparaginase treatment (1500 $\mathrm{IU} / \mathrm{kg}$ i.p. for 0-4 doses). Amylase was significantly increased in dexamethasone alone group, and was suppressed by asparaginase treatment (Figure B-9). Lipase was inhibited by asparaginase during the first week, but then rebound over time (Figure B-10), consistent with previous clinical report. ${ }^{62}$ Triglyceride level was directly correlated with lipase level (Figure B-11). There was no significant change in albumin level with increased asparaginase activity (Figure B-12). Plasma cortisol was increased by asparaginase treatment $(P=0.026)$ and suppressed by dexamethasone (Figure B-13).

We reviewed multiple clinical studies on the inhibitory effect of asparaginase treatment on protein synthesis, and summarized the data in Table B-3. Intriguingly, there was a strong association $(\mathrm{R}$-squared $=0.68, P<0.0001)$ between the percentage of inhibition and the content of asparagine residues of the protein (Figure B-14).

Almost all contemporary front-line ALL regimens contain glucocorticoids and asparaginase during induction therapy. The interaction between these two important antileukemic drugs can cause inter-individual variability in dexamethasone and asparaginase pharmacokinetics, which may influence the efficacy and toxicity of ALL treatment. This animal model allows us to study the optimal dosing strategy of glucocorticoids and asparaginase, and explore the utility of new biomarkers for osteonecrosis.

\section{Conclusion}

In summary, we were able to recapitulate the potentiating effect of asparaginase on glucocorticoid-induced osteonecrosis using a mouse model. Mice receiving asparaginase concomitantly with dexamethasone developed more arteriopathy and subsequent osteonecrosis than those received dexamethasone alone. The drug interaction should be considered when using glucocorticoids and asparaginase concomitantly in the treatment of ALL. 


\section{CHAPTER 4. ASPARAGINASE-INDUCED ACUTE PANCREATITIS}

\section{Introduction}

Acute pancreatitis is a major life-threatening complication of ALL treatment. Asparaginase has been recognized as the major cause of pancreatitis in children, adolescents, and adults being treated with combination chemotherapy for ALL, although steroids and thiopurines can also cause the complication. ${ }^{149-151}$ Acute pancreatitis occurs in 2-18\% of patients receiving asparaginase ${ }^{40,47-54}$ and is one of the common causes of asparaginase intolerance. Severe pancreatitis is a contraindication to further asparaginase therapy, leading to discontinuation of this agent. Because discontinuation of asparaginase treatment has been associated with compromised ALL treatment outcome, ${ }^{49,53}$ better understanding of risk factors for pancreatitis would provide insights towards therapy modifications that could decrease pancreatitis risk while maintaining effectiveness in patients with ALL.

The pathogenesis of asparaginase-induced pancreatitis has not been elucidated. Currently known clinical risk factors include intensive asparaginase therapy and older age $;^{47-50,56,65}$ however most studies lack sufficient power to robustly assess risk factors. ${ }^{4-8}$

Studies on pancreatitis of other etiologies, such as alcoholic, idiopathic and chronic pancreatitis, have identified a few genetic risk factors including cationic and anionic trypsinogen (PRSS1 and PRSS2), serine protease inhibitor kazal type 1 (SPINK1), cystic fibrosis trans-membrane conductance regulator $(C F T R)$, chymotrypsinogen $\mathrm{C}$ $(C T R C)$, calcium sensing receptor (CASR), claudin-2 (CLDN2), carboxypeptidase 1 $(C P A 1)$, and $H L A-D R B 1^{*} 07: 01$, most of which relate to premature trypsin activation or sustained trypsin activity in the pancreas. ${ }^{66,72,75-79,152,162}$ However, there has not been an agnostic genome-wide analysis of asparaginase-induced acute pancreatitis. ${ }^{82}$ Here we report the largest study to date to determine the clinical and genetic risk factors for acute pancreatitis in 5398 children, adolescents, and young adults treated for newly diagnosed ALL with multiagent chemotherapy regimens containing asparaginase.

\section{Methods}

\section{Patients and treatment}

We investigated 6171 children and young adults (age 0 to 30 years) with newly diagnosed ALL treated on seven front-line ALL protocols: Total XIIIB (NCI-T930101D) ${ }^{153}$, Total XV (NCT00137111) ${ }^{8}$, COG P9904 (NCT00005585), P9905 (NCT00005596), P9906 (NCT00005603) $)^{154}$, AALL0232 (NCT00075725) and AALL0331 (NCT00103285) at St. Jude Children's Research Hospital (SJCRH) and in the Children's Oncology Group (COG) between 1994 and 2011 (Table 4-1 and Figure 4-1). 
Table 4-1. Comparison of asparaginase regimens

\begin{tabular}{|c|c|c|c|c|c|c|c|}
\hline Protocol & Induction & Consolidation & $\begin{array}{l}\text { Interim Maintenance } \\
\text { (IM) and Delayed } \\
\text { Intensification (DI) }\end{array}$ & Maintenance & $\begin{array}{c}\text { Total } \\
\text { ASP } \\
\text { dose }^{b} \\
\left(\mathbf{U} / \mathbf{m}^{2}\right)\end{array}$ & $\begin{array}{c}\text { Total } \\
\text { ASP } \\
\text { weeks }\end{array}$ & $\begin{array}{c}\mathrm{N}(\%) \text { of } \\
\text { patients } \\
\text { developing } \\
\text { pancreatitis }\end{array}$ \\
\hline $\begin{array}{l}\text { SJCRH } \\
\text { Total XIIIB } \\
(1994-1998)\end{array}$ & $\begin{array}{l}\text { E. coli } 10000 \\
\mathrm{U} / \mathrm{m}^{2} \times 6\end{array}$ & No ASP & NA & $\begin{array}{l}\text { E. coli } 10000 \\
\mathrm{U} / \mathrm{m}^{2} \times 6\end{array}$ & 120000 & 4 & $\begin{array}{c}2 / 222 \\
(0.9 \%)\end{array}$ \\
\hline $\begin{array}{l}\text { SJCRH } \\
\text { Total XV } \\
(2000-2007)\end{array}$ & $\begin{array}{l}\text { E. coli } 10000 \\
\mathrm{U} / \mathrm{m}^{2} \times 6\end{array}$ & No ASP & NA & $\begin{array}{l}\text { Low risk: } E \text {. coli } \\
10000 \mathrm{U} / \mathrm{m}^{2} \times 18 \\
\text { Standard/high } \\
\text { risk: } E . \text { coli } 25000 \\
\mathrm{U} / \mathrm{m}^{2} \times 19\end{array}$ & $\begin{array}{c}240000- \\
535000\end{array}$ & $8-21$ & $\begin{array}{l}16 / 483 \\
(3.3 \%)\end{array}$ \\
\hline $\begin{array}{l}\text { COG } \\
\text { P9904/P9905 } \\
(2000-2007)\end{array}$ & $\begin{array}{l}\text { E. coli } 10000 \\
\mathrm{U} / \mathrm{m}^{2} \times 6^{\mathrm{d}} \text { or } \\
\mathrm{PEG} 2500 \\
\mathrm{U} / \mathrm{m}^{2} \times 1^{\mathrm{e}}\end{array}$ & $\begin{array}{l}\text { Randomized } \\
\text { to PEG } 2500 \\
\mathrm{U} / \mathrm{m}^{2} \times 1 \mathrm{vs} \text {. } \\
\text { no ASP }\end{array}$ & NA & No ASP & $\begin{array}{l}50000- \\
110000\end{array}$ & $2-4$ & $\begin{array}{l}12 / 1605 \\
(0.7 \%)\end{array}$ \\
\hline $\begin{array}{l}\text { COG P9906 } \\
(2000-2003)\end{array}$ & $\begin{array}{l}\text { E. coli } 10000 \\
\mathrm{U} / \mathrm{m}^{2} \times 6\end{array}$ & $\begin{array}{l}\text { E. coli } 6000 \\
\mathrm{U} / \mathrm{m}^{2} \times 12\end{array}$ & $\begin{array}{l}\text { E. coli } 15000 \mathrm{U} / \mathrm{m}^{2} \times 10 \\
\text { and } 6000 \mathrm{U} / \mathrm{m}^{2} \times 24\end{array}$ & No ASP & 420000 & 24 & $\begin{array}{l}11 / 222 \\
(5.0 \%)\end{array}$ \\
\hline \multirow[t]{2}{*}{$\begin{array}{l}\text { AALL0232 } \\
(2004-2011)\end{array}$} & $\begin{array}{l}\mathrm{PEG} 2500 \\
\mathrm{U} / \mathrm{m}^{2} \times 1\end{array}$ & $\begin{array}{l}\mathrm{PEG} 2500 \\
\mathrm{U} / \mathrm{m}^{2} \times 2\end{array}$ & $\begin{array}{l}\mathrm{DH} / \mathrm{PH}: \text { PEG } 2500 \\
\mathrm{U} / \mathrm{m}^{2} \times 2(\text { single IM/DI }) \\
\text { or } \times 6(\text { double IM/DI })\end{array}$ & No ASP & $\begin{array}{l}250000- \\
450000\end{array}$ & $10-18$ & $\begin{array}{l}32 / 1359 \\
(2.4 \%)\end{array}$ \\
\hline & & & $\begin{array}{l}\text { DC/PC: PEG } 2500 \\
\mathrm{U} / \mathrm{m}^{2} \times 4(\text { single IM/DI }) \\
\text { or } \times 6(\text { double IM/DI })\end{array}$ & & $\begin{array}{c}350000- \\
550000\end{array}$ & $14-22$ & $\begin{array}{c}44 / 1294 \\
(3.4 \%)\end{array}$ \\
\hline
\end{tabular}


Table 4-1. (Continued)

\begin{tabular}{|c|c|c|c|c|c|c|c|}
\hline Protocol & Induction & Consolidation & $\begin{array}{l}\text { Interim Maintenance } \\
\text { (IM) and Delayed } \\
\text { Intensification (DI) }\end{array}$ & Maintenance & $\begin{array}{c}\text { Total } \\
\text { ASP } \\
\text { dose }^{b} \\
\left(\mathbf{U} / \mathbf{m}^{2}\right)\end{array}$ & $\begin{array}{c}\text { Total } \\
\text { ASP } \\
\text { weeks }\end{array}$ & $\begin{array}{c}\mathrm{N}(\%) \text { of } \\
\text { patients } \\
\text { developing } \\
\text { pancreatitis }\end{array}$ \\
\hline \multirow[t]{2}{*}{$\begin{array}{l}\text { AALL0331 } \\
(2005-2010)\end{array}$} & \multirow[t]{2}{*}{$\begin{array}{l}\mathrm{PEG} 2500 \\
\mathrm{U} / \mathrm{m}^{2} \times 1\end{array}$} & \multirow{2}{*}{$\begin{array}{l}\text { Randomized } \\
\text { to PEG } 2500 \\
\mathrm{U} / \mathrm{m}^{2} \times 2 \mathrm{vs} \\
\text { no ASP }\end{array}$} & $\begin{array}{l}\text { Low/average risk: } \\
\text { Randomized to PEG } 2500 \\
\mathrm{U} / \mathrm{m}^{2} \times 1 \text { vs. } \times 4\end{array}$ & No ASP & $\begin{array}{l}100000- \\
350000\end{array}$ & $4-14$ & \multirow[t]{2}{*}{$\begin{array}{c}\text { NA } \\
\text { (Case-control } \\
\text { study) }\end{array}$} \\
\hline & & & $\begin{array}{l}\text { High risk: PEG } 2500 \\
\mathrm{U} / \mathrm{m}^{2} \times 8\end{array}$ & & $\begin{array}{c}450000- \\
550000\end{array}$ & $18-22$ & \\
\hline
\end{tabular}

All asparaginase was given intramuscularly. ${ }^{\mathrm{a}}$ Depends on patients' bone marrow blast percent during induction or minimal residual disease (MRD) status at the end of Induction. ${ }^{\mathrm{b}}$ Total dose and ${ }^{\mathrm{c}}$ total weeks of native E.coli asparaginase during therapy, excluded the extended Induction. PEG-asparaginase administration at $2500 \mathrm{U} / \mathrm{m}^{2}$ biweekly was considered therapeutically equivalent to native E.coli asparaginase at $25000 \mathrm{U} / \mathrm{m}^{2}$ weekly for two weeks. ${ }^{\mathrm{d}}$ Pre-amendment (before 10/07/2003), native E.coli asparaginase was used. ${ }^{\mathrm{e}}$ Post-amendment (from 10/07/2003), PEG-ASP was used. Abbreviations: ASP, asparaginase; E.coli, native E.coli asparaginase; PEG, pegylated E.coli asparaginase; DH/PH, dexamethasone/prednisone during induction therapy and high-dose methotrexate/leucovorin during the first interim maintenance; DC/PC, dexamethasone/prednisone during induction therapy and escalating methotrexate/leucovorin during the first interim maintenance. NA, not applicable. 


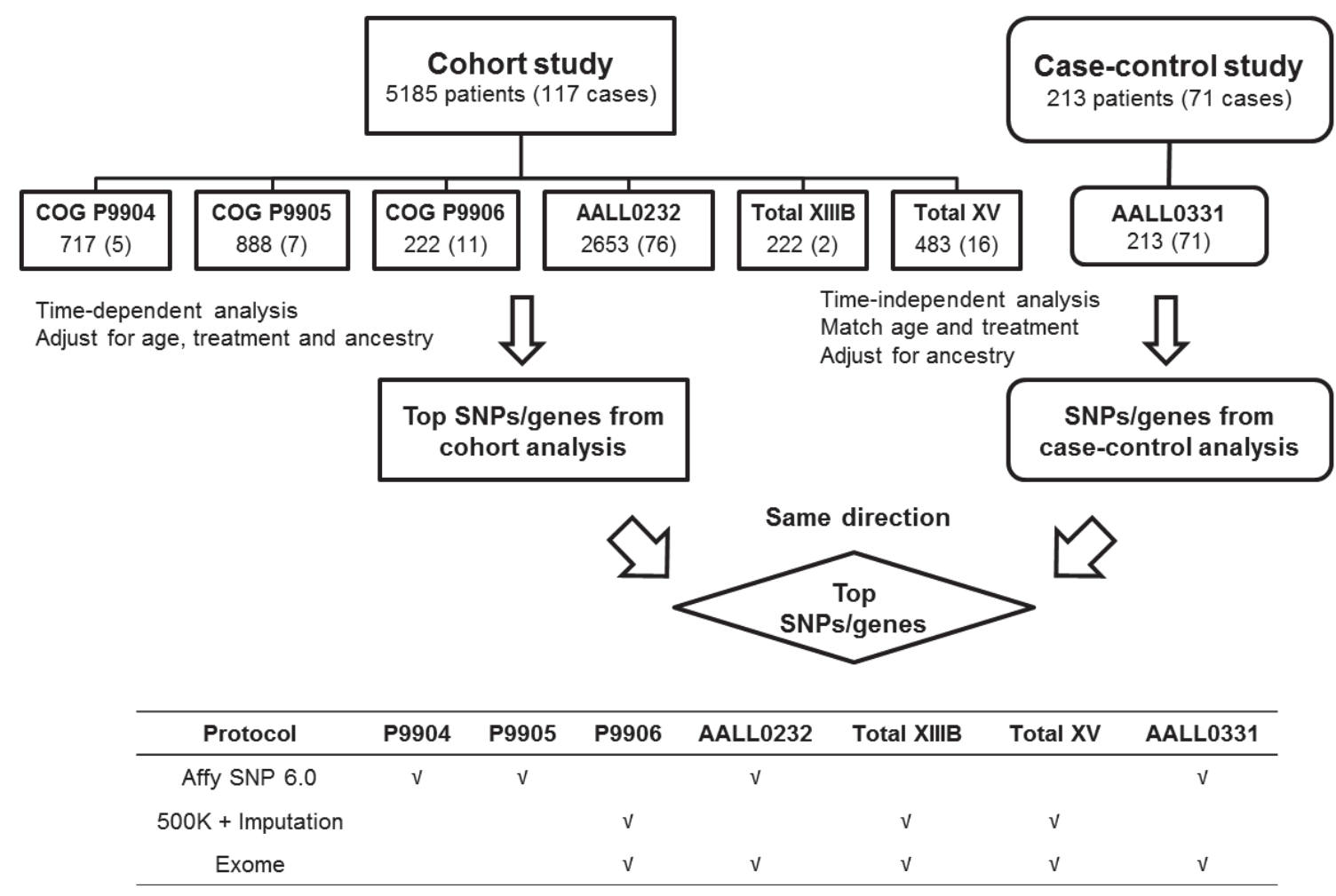

Figure 4-1. Study design

Study design and genotyping platforms of the initial GWAS (5398 patients) that aimed to identify the top ranked SNPs and genes from the cohort, and the SNPs or genes significantly associated with pancreatitis $(P<0.05)$ in the case-control group. The number of patients and number of cases (in parentheses) in each protocol are shown. 
The studies were approved by the institutional review boards of all participating institutions, and informed consent was obtained in accordance with the Declaration of Helsinki.

We studied 5185 of 5958 patients (87\%) enrolled on Total XIIIB/XV, COG P9904/P9905/P9906 and AALL0232 with available DNA, and a case-control group of 213 patients (71 cases and 142 controls) was selected from 5500 patients treated on AALL0331 (Table 4-2). In the case-control group, we studied every pancreatitis case on AALL0331 with available DNA and matched each case to two controls by age $( \pm 2$ years) and asparaginase treatment.

\section{Diagnosis of acute pancreatitis}

Acute pancreatitis was prospectively graded using NCI CTCAE version 3.0 for AALL0232 and AALL0331, and version 2.0 for all the other protocols. The diagnostic criteria included clinical symptoms (abdominal pain, nausea and vomiting), elevation in the serum amylase or lipase levels, and abnormal radiographic or surgical findings consistent with pancreatitis. For time-dependent analyses, time to first episode of pancreatitis was considered even if the patient had more than one episode of pancreatitis recorded.

\section{Genotyping}

Genotyping of SNPs was performed using the Illumina HumanExome Beadchips and either Affymetrix Genome-Wide Human SNP Array 6.0 or GeneChip Human Mapping 500K Array sets ${ }^{118}$ (Figure 4-1). This included a total of 5398 patients with Affymetrix Genome-Wide Human SNP 6.0 (or GeneChip Human Mapping 500K) Array data and 3469 patients with Illumina HumanExome Beadchips Array data. For those patients studied using the $500 \mathrm{~K}$ array sets, SNPs were imputed to include those on the Affymetrix SNP 6.0 array using MACH1.0. ${ }^{155}$ All patients in the cohort study $(\mathrm{n}=5185)$ were genotyped (or imputed) using SNP 6.0 array, while only a subset of patients $(\mathrm{n}=$ 3256) were genotyped using the Exome Beadchips. All patients in the case-control study $(\mathrm{n}=213)$ were genotyped on both arrays (Figure 4-1). The prediction of the potential damaging effect of SNPs was made using in silico prediction algorithms (SIFT, ${ }^{156}$ PolyPhen, ${ }^{157}$ LRT $^{158}$ and MutationTaster ${ }^{159}$ ). Combined Annotation Dependent Depletion (CADD) score ${ }^{160}$ was also used to estimate the deleteriousness of the coding SNPs (Table 4-5 and Figure C-1).

Genetic ancestries were determined using STRUCTURE, ${ }^{118}$ and either treated as a continuous variable or used to categorize patients as white, black, Hispanics, Asian, and "other" groups based on the percentage of European, African, Native American and Asian ancestries (Figure 4-2). 
Table 4-2. Number of patients included in this study

\begin{tabular}{llccc}
\hline Study & \multicolumn{1}{c}{ Protocol } & $\begin{array}{c}\text { N of } \\
\text { patients }\end{array}$ & $\begin{array}{c}\text { N (\%) with } \\
\text { array data }^{\text {a }}\end{array}$ & $\begin{array}{c}\text { N (\%) with deep } \\
\text { sequencing data }^{\text {b }}\end{array}$ \\
\hline Cohort study & COG P9904/P9905 & 1883 & $1605(85.2 \%)$ & $1359(72.2 \%)$ \\
& COG P9906 & 272 & $222(81.6 \%)$ & $158(58.1 \%)$ \\
& AALL0232 & 3058 & $2653(86.7 \%)$ & $2030(66.3 \%)$ \\
& Total XIIIB & 247 & $222(89.9 \%)$ & $213(86.2 \%)$ \\
& Total XV & 498 & $483(96.9 \%)$ & $457(91.8 \%)$ \\
$\begin{array}{l}\text { Case-control } \\
\text { study }\end{array}$ & AALL0331 & 5958 & $5185(87.0 \%)$ & $4217(70.8 \%)$ \\
\hline
\end{tabular}

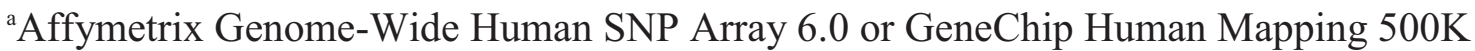
Array sets. ${ }^{\text {b }} 283$ genes were sequenced, including 42 putative pancreatitis candidate genes based on literatures and the initial GWAS using the array data. 


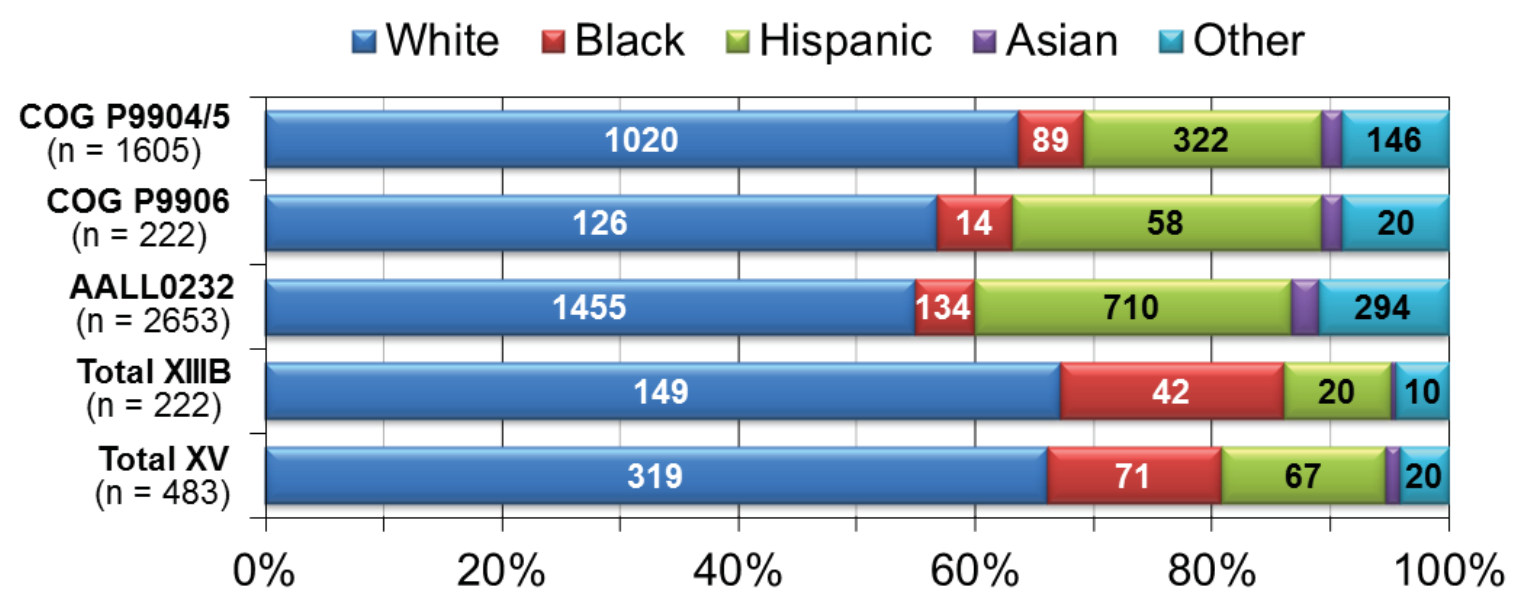

Figure 4-2. Race group assignments based on the ancestral composition of patients in the cohort $(n=5185)$ by treatment protocol

Number of patients of each ancestry (except Asian) are shown on the bars. Genetic ancestry was estimated using STRUCTURE, ${ }^{118}$ and patients were assigned to race groups categorized as white (European ancestry $>90 \%$ ), black (African ancestry $>70 \%$ ), Hispanic (Native American ancestry $>10 \%$ ), Asian (Asian ancestry $>90 \%$ ) and "other". 


\section{SNP-based analysis for pancreatitis}

After excluding the SNPs with a call rate $<95 \%, 751455$ of 931947 SNPs on the SNP 6.0 array and 169521 of 174435 SNPs on the Exome Beadchip were interrogated in the initial GWAS. Each SNP was first individually tested using the Gray test and those achieving $P<0.05$ were subsequently analyzed. Genotype calls were coded as 0,1 or 2 for the number of $\mathrm{B}$ alleles $(\mathrm{AA}, \mathrm{AB}$ or $\mathrm{BB})$ assuming an additive genetic model. In the cohort $(n=5185)$, GWAS was performed using a Cox proportional hazard model considering the onset time of pancreatitis (time-dependent GWAS). Covariates included age (continuous variable in years), genetic ancestry (continuous variable as percentages of European, African, Asian and Native American ancestries), and treatment regimen (protocol-planned cumulative asparaginase dose $\leq 120000 \mathrm{U} / \mathrm{m}^{2} \mathrm{vs} . \geq 240000 \mathrm{U} / \mathrm{m}^{2}$ as a categorical variable). For patients with pancreatitis, their time-at-risk was censored at the first episode of pancreatitis; for those without pancreatitis, their time-at-risk was censored at off-study date. In the case-control group $(n=213)$, controls were matched to cases for age and treatment regimen, and GWAS was performed using logistic regression, adjusted for genetic ancestry only (time-independent GWAS).

\section{Gene-level analysis of nonsense variants using SKAT}

SNPs were assigned to genes using Refseq boundaries (UCSC Genome Browser, hg19 assembly). For each gene, sequence kernel association test ${ }^{161}$ (SKAT) was applied to test for gene level significance for the effects of nonsense variants. For time dependent analysis, Martingale residuals as a continuous phenotype in the SKAT analysis were determined after applying Cox proportional hazard model using demographic and clinical covariates, including age, genetic ancestry, and treatment regimen.

\section{Deep sequencing of selected genes}

To interrogate rare and low-frequency variants in genes of interest for ALLrelated phenotypes, we sequenced 283 genes in 4217 children in the cohort and 162 in the case-control group (Table 4-2). The 283 genes were selected for sequencing because they were candidate genes for a number of ALL phenotypes (e.g. relapse, drug sensitivity, pharmacokinetics, leukemia risk, and several adverse effects); 42 of these were possible pancreatitis risk genes (Table 4-3). For more details of deep sequencing, please see Appendix C.

Gene-level analysis of all 283 genes and single variant analysis of 9229 SNPs in the 42 pancreatitis-associated genes was performed using the same methods as in the initial GWAS (see Appendix C). 
Table 4-3. Putative genes associated with pancreatitis $(n=42)$ selected for deep sequencing based on GWAS result or literature review

\begin{tabular}{|c|c|c|c|}
\hline Gene & Official full name & Function* & Source \\
\hline ADAMTS18 & $\begin{array}{l}\text { ADAM metallopeptidase with } \\
\text { thrombospondin type } 1 \text { motif, } 18\end{array}$ & Tumor suppressor & GWAS \\
\hline$A G E R$ & $\begin{array}{l}\text { Advanced glycosylation end } \\
\text { product-specific receptor }\end{array}$ & $\begin{array}{l}\text { Member of the immunoglobulin superfamily of cell surface } \\
\text { receptors implicated in homeostasis, development and } \\
\text { inflammation, and certain diseases, such as diabetes and } \\
\text { Alzheimer's disease }\end{array}$ & GWAS \\
\hline$A L S 2 C L$ & ALS2 C-terminal like & $\begin{array}{l}\text { Guanine nucleotide exchange factor (GEF) for Rab5 GTPase } \\
\text { regulating the ALS2-mediated endosome dynamics }\end{array}$ & GWAS \\
\hline C11orf63 & $\begin{array}{l}\text { Chromosome } 11 \text { open reading } \\
\text { frame } 63\end{array}$ & Protein-coding gene & GWAS \\
\hline C12orf40 & $\begin{array}{l}\text { Chromosome } 12 \text { open reading } \\
\text { frame } 40\end{array}$ & Protein-coding gene & GWAS \\
\hline C9orf117 & $\begin{array}{l}\text { Chromosome } 9 \text { open reading } \\
\text { frame } 117\end{array}$ & Protein-coding gene & GWAS \\
\hline$C A S R$ & Calcium-sensing receptor & $\begin{array}{l}\text { G protein-coupled receptor that senses small changes in } \\
\text { circulating calcium concentration and couples this information } \\
\text { to intracellular signaling pathways }\end{array}$ & Literature $^{72}$ \\
\hline CD52 & Cluster of differentiation 52 & Possible role in carrying and orienting carbohydrate & GWAS \\
\hline CFTR & $\begin{array}{l}\text { Cystic fibrosis transmembrane } \\
\text { conductance regulator }\end{array}$ & $\begin{array}{l}\text { cAMP-regulated epithelial cell channel involved in fluid } \\
\text { transport }\end{array}$ & Literature $^{153,162}$ \\
\hline CLDN2 & Claudin 2 & $\begin{array}{l}\text { Major integral membrane protein localized exclusively at tight } \\
\text { junction }\end{array}$ & Literature $^{66,69}$ \\
\hline CPA1 & $\begin{array}{l}\text { Carboxypeptidase A1 } \\
\text { (pancreatic) }\end{array}$ & $\begin{array}{l}\text { A1 form of the pancreatic procarboxypeptidase produced in } \\
\text { the pancreas and preferentially cleaves C-terminal branched- } \\
\text { chain and aromatic amino acids from dietary proteins }\end{array}$ & Literature $^{80,81}$ \\
\hline
\end{tabular}




\section{Table 4-3. (Continued)}

\begin{tabular}{|c|c|c|c|}
\hline Gene & Official full name & Function* & Source \\
\hline CPA2 & $\begin{array}{l}\text { Carboxypeptidase A2 } \\
\text { (pancreatic) }\end{array}$ & $\begin{array}{l}\text { A2 form of pancreatic procarboxypeptidase produced in the } \\
\text { pancreas and preferentially cleaves aromatic C-terminal } \\
\text { residues from dietary proteins }\end{array}$ & GWAS \\
\hline CTRC & Chymotrypsin C & $\begin{array}{l}\text { Serum calcium-decreasing factor with chymotrypsin-like } \\
\text { protease activity, regulating activation and degradation of } \\
\text { trypsinogens and procarboxypeptidases by targeting specific } \\
\text { cleavage sites within their zymogen precursors }\end{array}$ & Literature $^{162-164}$ \\
\hline$D D X 49$ & $\begin{array}{l}\text { DEAD (Asp-Glu-Ala-Asp) box } \\
\text { polypeptide } 49\end{array}$ & RNA binding and ATP-dependent helicase activity & GWAS \\
\hline DOCK5 & Dedicator of cytokinesis 5 & $\begin{array}{l}\text { Guanine nucleotide exchange factor (GEF) for Rho and Rac } \\
\text { which regulates of actin distribution and amylase secretion in } \\
\text { pancreatic acinar cells }\end{array}$ & GWAS \\
\hline FHIT & Fragile histidine triad & $\begin{array}{l}\text { Diadenosine } 5 \text { ',5"'-P1,P3-triphosphate hydrolase involved in } \\
\text { purine metabolism }\end{array}$ & GWAS \\
\hline$G C K R$ & $\begin{array}{l}\text { Glucokinase (hexokinase 4) } \\
\text { regulator }\end{array}$ & $\begin{array}{l}\text { Inhibiting glucokinase in liver and pancreatic islet cells by } \\
\text { binding non-covalently to form an inactive complex with the } \\
\text { enzyme }\end{array}$ & GWAS \\
\hline HOGA1 & $\begin{array}{l}\text { 4-hydroxy-2-oxoglutarate } \\
\text { aldolase } 1\end{array}$ & $\begin{array}{l}\text { Catalyzing the final step in the metabolic pathway of } \\
\text { hydroxyproline, releasing glyoxylate and pyruvate }\end{array}$ & GWAS \\
\hline$H S D 17 B 2$ & $\begin{array}{l}\text { Hydroxysteroid (17-beta) } \\
\text { dehydrogenase } 2\end{array}$ & $\begin{array}{l}\text { Capable of catalyzing the interconversion of testosterone and } \\
\text { androstenedione, as well as estradiol and estrone }\end{array}$ & GWAS \\
\hline IFNA5 & Interferon, alpha 5 & Cytokine activity and antiviral activity & GWAS \\
\hline$I N M T$ & $\begin{array}{l}\text { Indolethylamine N- } \\
\text { methyltransferase }\end{array}$ & Catalyzing N-methylation of indoles such as tryptamine & GWAS \\
\hline ITFG1 & $\begin{array}{l}\text { Integrin alpha FG-GAP repeat } \\
\text { containing } 1\end{array}$ & Modulator of T-cell function & GWAS \\
\hline KLHDC1 & Kelch domain containing 1 & Protein-coding gene, paralog of $H C F C 2$ (Host Cell Factor C2) & GWAS \\
\hline
\end{tabular}




\section{Table 4-3. (Continued)}

\begin{tabular}{|c|c|c|c|}
\hline Gene & Official full name & Function* & Source \\
\hline LRRC39 & $\begin{array}{l}\text { Leucine rich repeat containing } \\
39\end{array}$ & $\begin{array}{l}\text { Protein-coding gene, paraglog of } L R S A M 1 \text {, which is a } \\
\text { regulator of signaling pathways of cell adhesion, mediating } \\
\text { self-ubiquitylation and cargo sorting during receptor } \\
\text { endocytosis }\end{array}$ & GWAS \\
\hline MICAL2 & $\begin{array}{l}\text { Microtubule associated } \\
\text { monooxygenase, calponin and } \\
\text { LIM domain containing } 2\end{array}$ & $\begin{array}{l}\text { Nuclear monooxygenase that promotes depolymerization of F- } \\
\text { actin, and interacts with small GTPase Rab1, which plays a } \\
\text { role in vesicle trafficking from the endoplasmic reticulum to } \\
\text { the Golgi }\end{array}$ & GWAS \\
\hline$M P Z L 3$ & Myelin protein zero-like 3 & Mediator of homophilic cell-cell adhesion & GWAS \\
\hline$N M I$ & N-myc (and STAT) interactor & $\begin{array}{l}\text { May be involved in augmenting coactivator protein } \\
\text { recruitment to a group of sequence-specific transcription } \\
\text { factors. Augments cytokine-mediated STAT transcription }\end{array}$ & GWAS \\
\hline PHKB & Phosphorylase kinase, beta & $\begin{array}{l}\text { Regulatory unit of phosphorylase kinase B that activates } \\
\text { cAMP-dependent phosphorylase kinase pathway }\end{array}$ & GWAS \\
\hline PRSS1 & Protease, serine, 1 (trypsin 1$)$ & $\begin{array}{l}\text { Also known as cationic trypsinogen, a pancreatic serine } \\
\text { protease that is active on peptide linkages involving the } \\
\text { carboxyl group of lysine or arginine }\end{array}$ & Literature $^{66,69,162}$ \\
\hline PRSS2 & Protease, serine, 2 (trypsin 2$)$ & $\begin{array}{l}\text { Also known as anionic trypsinogen, a pancreatic serine } \\
\text { protease that is active on peptide linkages involving the } \\
\text { carboxyl group of lysine or arginine }\end{array}$ & Literature $^{66,69}$ \\
\hline$R A N B P 10$ & RAN binding protein 10 & $\begin{array}{l}\text { Guanine nucleotide exchange factor (GEF) for RAN GTPase. } \\
\text { It plays an essential role in hemostasis and in maintaining } \\
\text { microtubule dynamics, and enhances dexamethasone-induced } \\
\text { transactivation activity of glucocorticoid receptor NR } 3 \mathrm{C} 1\end{array}$ & GWAS \\
\hline RUFY4 & $\begin{array}{l}\text { RUN and FYVE domain } \\
\text { containing } 4\end{array}$ & $\begin{array}{l}\text { Protein-coding gene, paralog of FYCO1 (FYVE And Coiled- } \\
\text { Coil Domain Containing 1) that mediates microtubule plus } \\
\text { end-directed vesicle transport }\end{array}$ & GWAS \\
\hline
\end{tabular}




\section{Table 4-3. (Continued)}

\begin{tabular}{|c|c|c|c|}
\hline Gene & Official full name & Function* & Source \\
\hline SCAMP3 & $\begin{array}{l}\text { Secretory carrier membrane } \\
\text { protein } 3\end{array}$ & $\begin{array}{l}\text { Secretory carrier membrane protein that functions as a carrier } \\
\text { to the cell surface in post-golgi recycling pathways, and } \\
\text { involves in protein trafficking in endosomal pathways }\end{array}$ & GWAS \\
\hline SERPINA9 & $\begin{array}{l}\text { Serpin peptidase inhibitor, clade } \\
\text { A (alpha-1 antiproteinase, } \\
\text { antitrypsin), member } 9\end{array}$ & $\begin{array}{l}\text { Protease inhibitor that inhibits trypsin and trypsin-like serine } \\
\text { proteases }\end{array}$ & GWAS \\
\hline SLC6A18 & $\begin{array}{l}\text { Solute carrier family } 6 \text { (neutral } \\
\text { amino acid transporter), member } \\
18\end{array}$ & $\begin{array}{l}\text { Renal transporter for neurotransmitters, amino acids, and } \\
\text { osmolytes like betaine, taurine, and creatine }\end{array}$ & GWAS \\
\hline SNTG2 & Syntrophin, gamma 2 & $\begin{array}{l}\text { Interacting with mechanosensitive sodium channel that affects } \\
\text { channel gating }\end{array}$ & GWAS \\
\hline$S P A G 8$ & Sperm associated antigen 8 & $\begin{array}{l}\text { Involved in spermatogenesis by regulating microtubule } \\
\text { formation and cell division }\end{array}$ & GWAS \\
\hline SPINK1 & $\begin{array}{l}\text { Serine peptidase inhibitor, Kazal } \\
\text { type } 1\end{array}$ & $\begin{array}{l}\text { Prevention of trypsin-catalyzed premature activation of } \\
\text { zymogens within the pancreas and the pancreatic duct }\end{array}$ & Literature $^{71,72,162}$ \\
\hline TRAP1 & TNF receptor-associated protein & Regulating cellular stress responses & GWAS \\
\hline ZNF233 & Zinc finger protein 233 & $\begin{array}{l}\text { Protein-coding gene that may be involved in transcriptional } \\
\text { regulation }\end{array}$ & GWAS \\
\hline ZNF701 & Zinc finger protein 701 & $\begin{array}{l}\text { Protein-coding gene that may be involved in transcriptional } \\
\text { regulation }\end{array}$ & GWAS \\
\hline ZRANB3 & $\begin{array}{l}\text { Zinc finger, RAN-binding } \\
\text { domain containing } 3\end{array}$ & $\begin{array}{l}\text { DNA annealing helicase and endonuclease required to } \\
\text { maintain genome stability at stalled or collapsed replication } \\
\text { forks }\end{array}$ & GWAS \\
\hline
\end{tabular}

*Functional annotation obtained from NCBI Gene database (http://www.ncbi.nlm.nih.gov/gene), Uniprot (http://www.uniprot.org) and Genecards (www.genecards.org). Genes involved in cytoskeleton regulation and vesicle trafficking pathways are highlighted. 


\section{Statistical analysis}

All statistical analyses were conducted using R (version 3.1.0). Demographics were compared using Chi square test or Wilcoxon rank sum test. Event-time distributions were analyzed using the Kaplan-Meier method, and compared using the log-rank test. Multivariate Cox proportional hazard model was used to determine the potential risk factors for acute pancreatitis. Classification and regression tree (CART) analysis was performed with the ctree function of the package party $^{165}$ of $\mathrm{R}$ (version 3.1.0).

\section{Results}

\section{Pancreatitis associated with asparaginase}

Among the 5185 patients in the cohort, $117(2.3 \%)$ were diagnosed with at least one episode of acute pancreatitis during therapy. The frequency differed by protocols: Total XIIIB (0.9\%, 2/222), Total XV (3.3\%, 16/483), P9904 (0.7\%, 5/717), P9905 (0.8\%, 7/888), P9906 (5.0\%, 11/222) and AALL0232 (2.9\%, 76/2653; Figure 4-3A). The first episode of pancreatitis occurred at a median of 106 days from ALL diagnosis, and developed during remission induction (first 4-6 weeks of treatment) in 24 of 117 (20.5\%) patients and during the first year of therapy (including remission induction) in 104 of 117 (88.9\%) patients (Figure 4-3A). The great majority of the events were clustered with asparaginase administration (Figure 4-4).

Among the 71 pancreatitis cases treated on AALL0331 (case-control group), the first episode of pancreatitis occurred at a median of 78 days, and developed during remission induction in 24 of $71(33.8 \%)$ cases and during the first year in 65 of 71 $(91.5 \%)$ cases.

The risk of pancreatitis was related to cumulative dose (R-squared $=0.77, P=1.8$ $\left.\times 10^{-4}\right)$ and duration of asparaginase treatment $\left(\mathrm{R}\right.$-squared $=0.69, P=8.9 \times 10^{-4}$; Figure 4-3B and C). Because asparaginase dose and duration were highly correlated (R-squared $=0.86, P=1.6 \times 10^{-5}$ ), it was not possible to determine which was more important for pancreatitis risk.

\section{Clinical risk factors}

To identify covariates to include in the GWAS, we tested for the associations between pancreatitis and clinical characteristics in the cohort (Figure 4-5). Patients with pancreatitis were older $\left(11.7 \pm 5.1\right.$ years vs. $8.6 \pm 5.6$ years; $\left.P=2.2 \times 10^{-9}\right)$, and the incidence was related to age as a continuous variable in univariate analysis $\left(P=1.3 \times 10^{-}\right.$ $\left.{ }^{7}\right)$. Hispanic $(3.9 \%)$ and black (3.1\%) patients had higher risk than white $(1.5 \%)$ and Asian $(1.0 \%)$ patients. We categorized the protocols into low-dose $\left(\leq 120000 \mathrm{U} / \mathrm{m}^{2}\right)$ and high-dose asparaginase regimens $\left(\geq 240000 \mathrm{U} / \mathrm{m}^{2}\right.$; Table 4-1). High-dose regimens had 

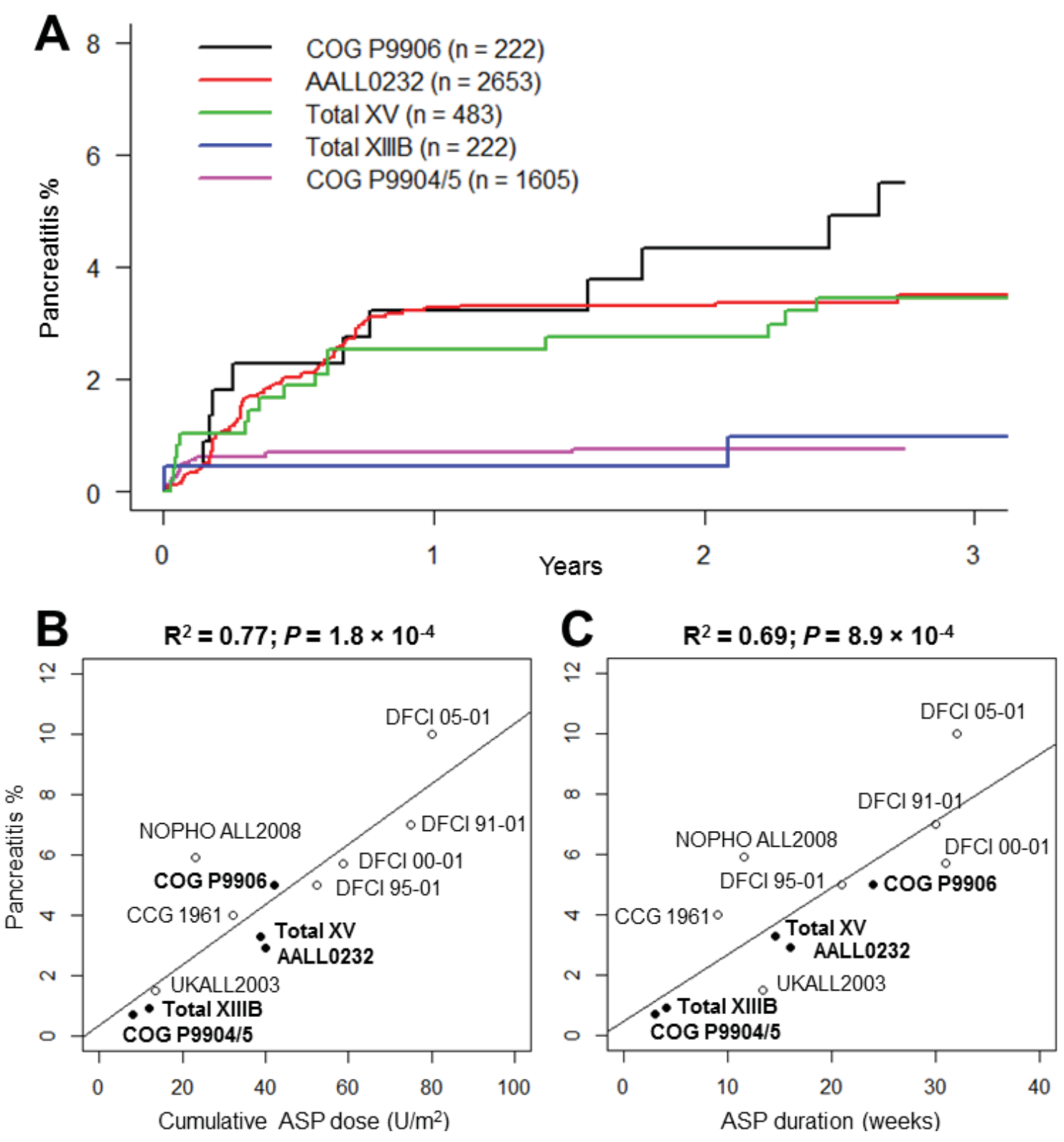

Figure 4-3. Incidence of pancreatitis differed by protocols

(A) Cumulative incidence of pancreatitis in the cohort $(n=5185)$ by different protocols.

(B) The relationship between incidence of pancreatitis and cumulative dose of ASP and

(C) the relationship between incidence and treatment duration of ASP in this study (filled circles) and in some recently reported protocols ${ }^{49,50,52,53}$ (empty circles). The individual points show the protocol-defined dose or treatment duration of native E. coli-ASP across all treatment arms for each protocol. PEG-ASP $2500 \mathrm{U} / \mathrm{m}^{2}$ biweekly is considered equivalent to native $E$. coli-ASP at $25000 \mathrm{U} / \mathrm{m}^{2}$ weekly for two weeks. The linear regression lines, R-squared and $\mathrm{P}$ values are shown. ASP, asparaginase. 
Figure 4-4. Time of pancreatitis in the cohort $(n=5185)$ during the first year of therapy

The first episode of pancreatitis (red stars) and asparaginase administration (arrows) are shown. DI, delayed intensification; $\mathrm{DH} / \mathrm{PH}$, dexamethasone/prednisone and high-dose methotrexate arm; DC/PC, dexamethasone/prednisone and Capizzi (escalating) methotrexate arm; LR, low-risk; SHR, standard/high-risk; PEG-ASP, pegylated E.coli asparaginase; IM, interim maintenance. 
Study (N of cases in the first year/N of patients)

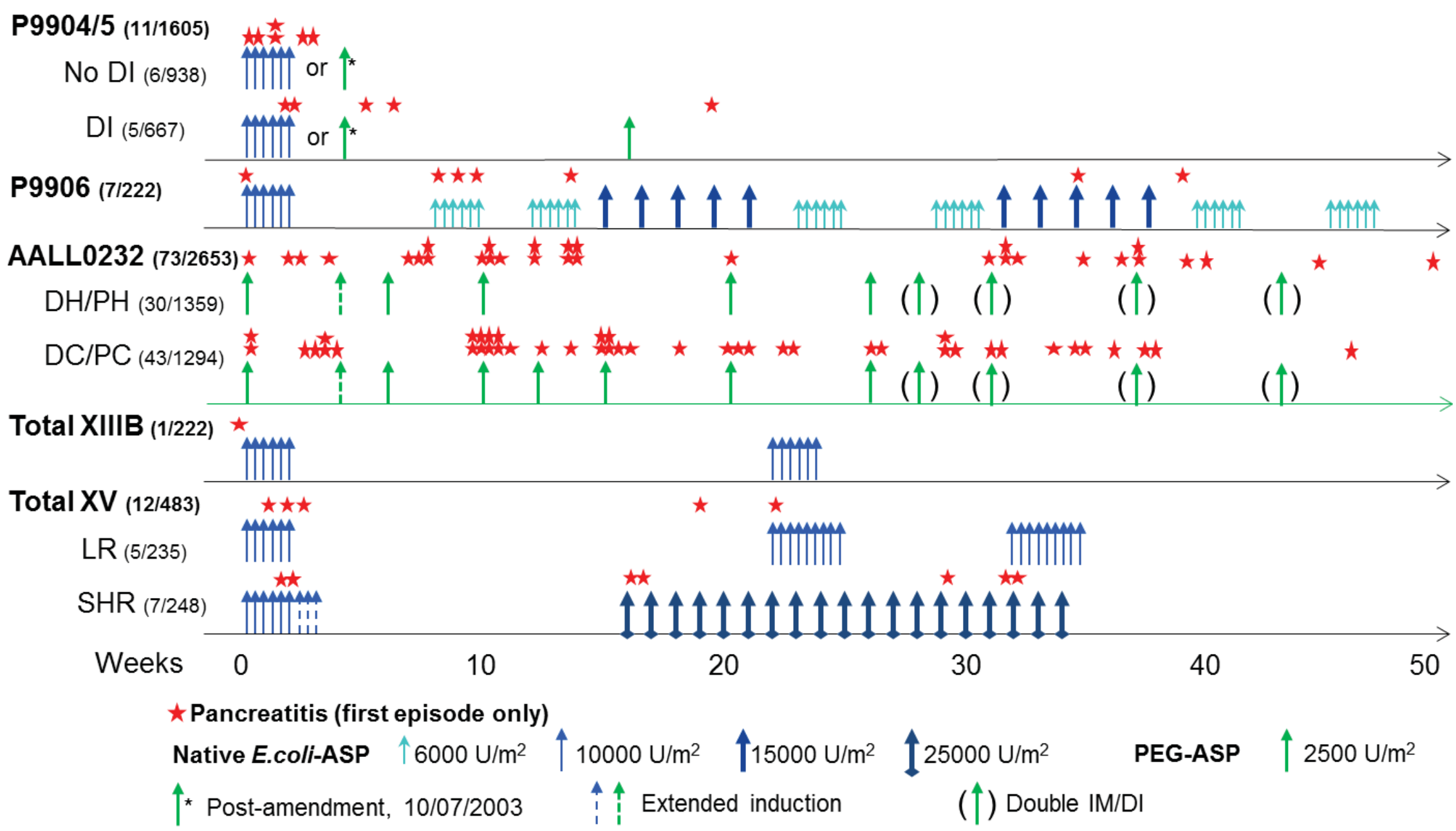


Age (years)

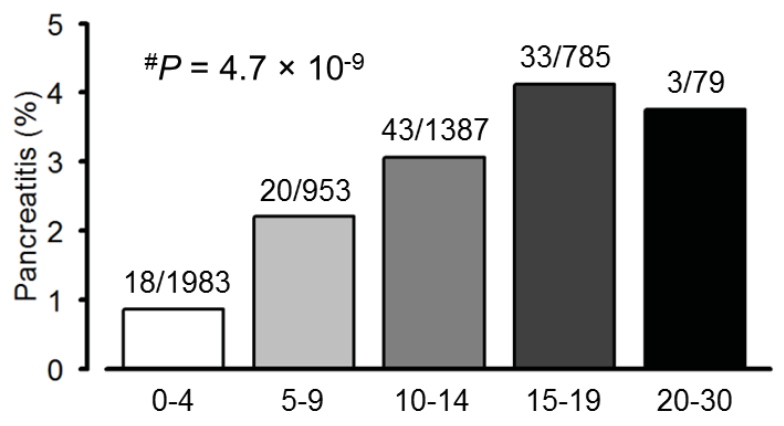

Race group (genetically assigned)

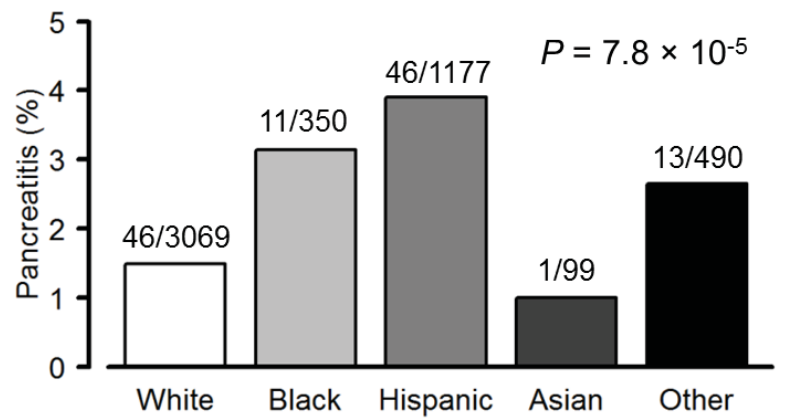

Gender

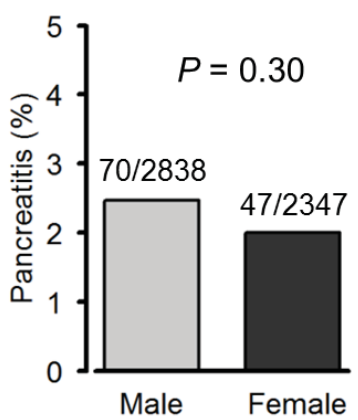

Cumulative ASP dose

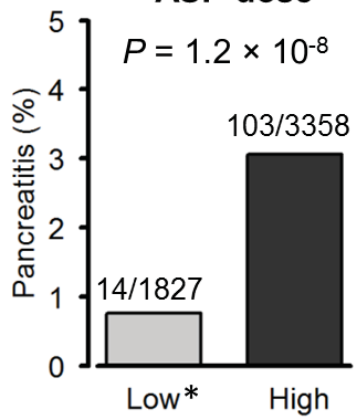

ALL lineage

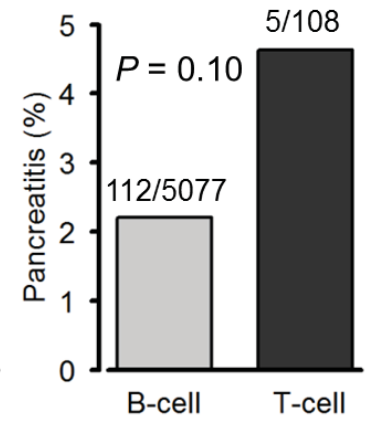

ASP formulation

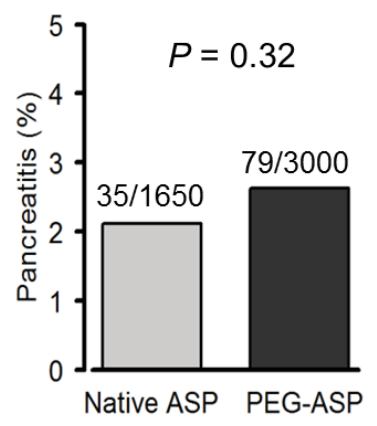

Figure 4-5. The incidence of pancreatitis based on clinical characteristics *Low-dose asparaginase ( $\leq 120000 \mathrm{U} / \mathrm{m}^{2}$, including P9904, P9905 and Total XIIIB) and high-dose asparaginase regimens $\left(\geq 240000 \mathrm{U} / \mathrm{m}^{2}\right.$, including P9906, AALL0232 and Total XV). Chi-square $\mathrm{P}$ values for categorical variables and ${ }^{\#} \mathrm{P}$ value from linear regression model for continuous variable (age) are shown. ASP, asparaginase. 
more pancreatitis $\left(3.1 \%\right.$ vs. $\left.0.8 \%, P=1.2 \times 10^{-8}\right)$. These features remained as independent risk factors in a multivariate model (Table 4-4). Interestingly, there was a direct correlation between the percentage of Native American ancestry and a higher risk of pancreatitis (Figure 4-6). Only P9904/P9905 included some patients treated with one or the other formulation of asparaginase (Table 4-1), and the incidence of pancreatitis did not differ between those who received native E.coli asparaginase alone $(0.8 \%, 6 / 723)$, PEG-asparaginase alone $(0.9 \%, 3 / 347)$ or both $(0.6 \%, 3 / 535)$.

A CART analysis was also used to depict the clinical risk factors in order of importance (Figure 4-7). The covariates included in the model are ancestry, age, lineage, gender, asparaginase regimen and formulation. The most important factor for pancreatitis is high exposure to asparaginase. For children on a low-dose regimen $\left(\leq 120000 \mathrm{U} / \mathrm{m}^{2}\right)$, those older than 10 years of age had a higher risk $(2.2 \%, 6 / 267)$ than those younger than 10 years of age $(0.5 \%, 8 / 1560 ; P=0.01)$. For those on a high-dose regimen $(\geq 240000$ $\left.\mathrm{U} / \mathrm{m}^{2}\right)$, Hispanic and black children had a higher risk $(4.9 \%, 52 / 1054)$ than other race groups $\left(2.2 \%, 51 / 2304 ; P=5.2 \times 10^{-5}\right)$.

Thus we used age, ancestry and treatment regimen as covariates in all genomic analysis in the cohort, and ancestry in the case-control group.

\section{Gene-level analysis of nonsense variants identified $C P A 2$}

Focusing on rare variants and nonsense variants, six genes were associated with pancreatitis in the cohort at the $P<0.05$ level that were also associated with pancreatitis in the case-control group (Table 4-5). In the cohort, $C P A 2$, with a nonsense SNP at rs199695765 showed the strongest association $\left(P=2.1 \times 10^{-9}\right)$. The $\mathrm{C}>\mathrm{T}$ SNP at rs199695765 was predicted to cause early termination of the CPA2 protein (Figure 4-8A). Two of 3469 patients carried this variant (one in the cohort and one in the case/control group) and both developed early pancreatitis (Figure 4-8B); one of them had recurrent episodes after each of two asparaginase re-exposures.

\section{GWAS of common SNPs}

Analyzing all coding and non-coding common SNPs (minor allele frequency $[\mathrm{MAF}] \geq 1 \%)$ in the cohort, we identified loci in a gene involved in purine metabolism, FHIT (rs9849262, $P=8.4 \times 10^{-6}$ and rs9859201, $P=1.2 \times 10^{-4}$ ). Among the top 20 common SNPs, 14 were assigned to 7 genes, and 7 of the 14 SNPs were in 3 genes involved in cytoskeleton regulation, including DOCK5, ACTN2, and MICAL2 (Table 4-6). These SNPs were also associated with higher risk in the case-control study, although some of them did not reach $P<0.05$ in this relatively small group. Results for top ranked variants from single SNP analyses, including nonsense SNPs, missense SNPs, and synonymous and non-coding SNPs are shown in Appendix C (Tables C-1, C-2 and C-3). 
Table 4-4. Multivariate analysis for clinical risk of pancreatitis in the cohort study $(\mathrm{n}=\mathbf{5 1 8 5})$

\begin{tabular}{lcc}
\hline Patient characteristics & $\boldsymbol{P}$ & Odds ratio (95\% CI) \\
\hline Native American ancestry $(+10 \%)$ & $7.6 \times 10^{-8}$ & $1.2(1.1-1.3)$ \\
Age (years) & $1.2 \times 10^{-5}$ & $1.1(1.0-1.1)$ \\
High-dose ASP regimen $^{\S}$ & $1.1 \times 10^{-4}$ & $3.3(1.8-5.9)$ \\
African ancestry (+10\%) & 0.048 & $1.1(1.0-1.2)$ \\
Native E.coli ASP only & 0.14 & $1.4(0.9-2.2)$ \\
T-cell lineage ALL & 0.27 & $1.7(0.7-4.5)$ \\
Asian ancestry (+10\%) & 0.60 & $1.0(0.9-1.2)$ \\
Male gender & 0.68 & $1.1(0.7-1.6)$ \\
\hline
\end{tabular}

Association with risk of pancreatitis was assessed using Cox proportional hazard model. Age and ancestry were treated as continuous variables; e.g. an odds ratio of 1.2 suggests 1.2 fold higher risk of pancreatitis for every $10 \%$ increase in Native American ancestry. Other characteristic features are dichotomized for presence versus absence of the indicated feature. ${ }^{\S}$ Total protocol-specified E.coli asparaginase dose $\geq 240000 \mathrm{U} / \mathrm{m}^{2}$ (i.e. all patients on Total XV, COG P9906 and AALL0232) compared to $\leq 120000 \mathrm{U} / \mathrm{m}^{2}$. 


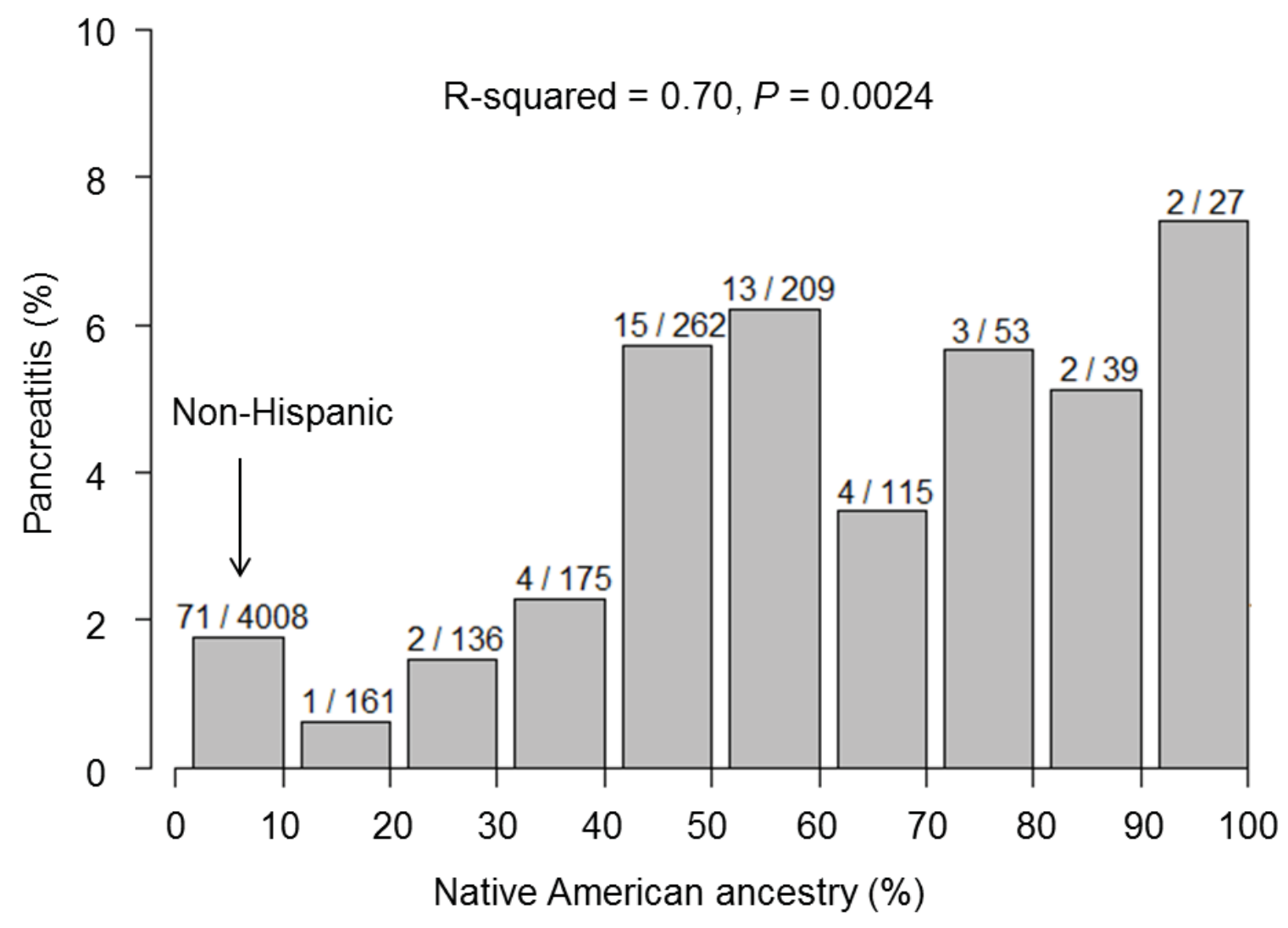

Figure 4-6. Association between Native American ancestry and pancreatitis The distribution of patients with pancreatitis and the total number of patients by the percentage of Native American ancestry (in 10\% interval). R-squared and P value were calculated using a univariate linear model. 


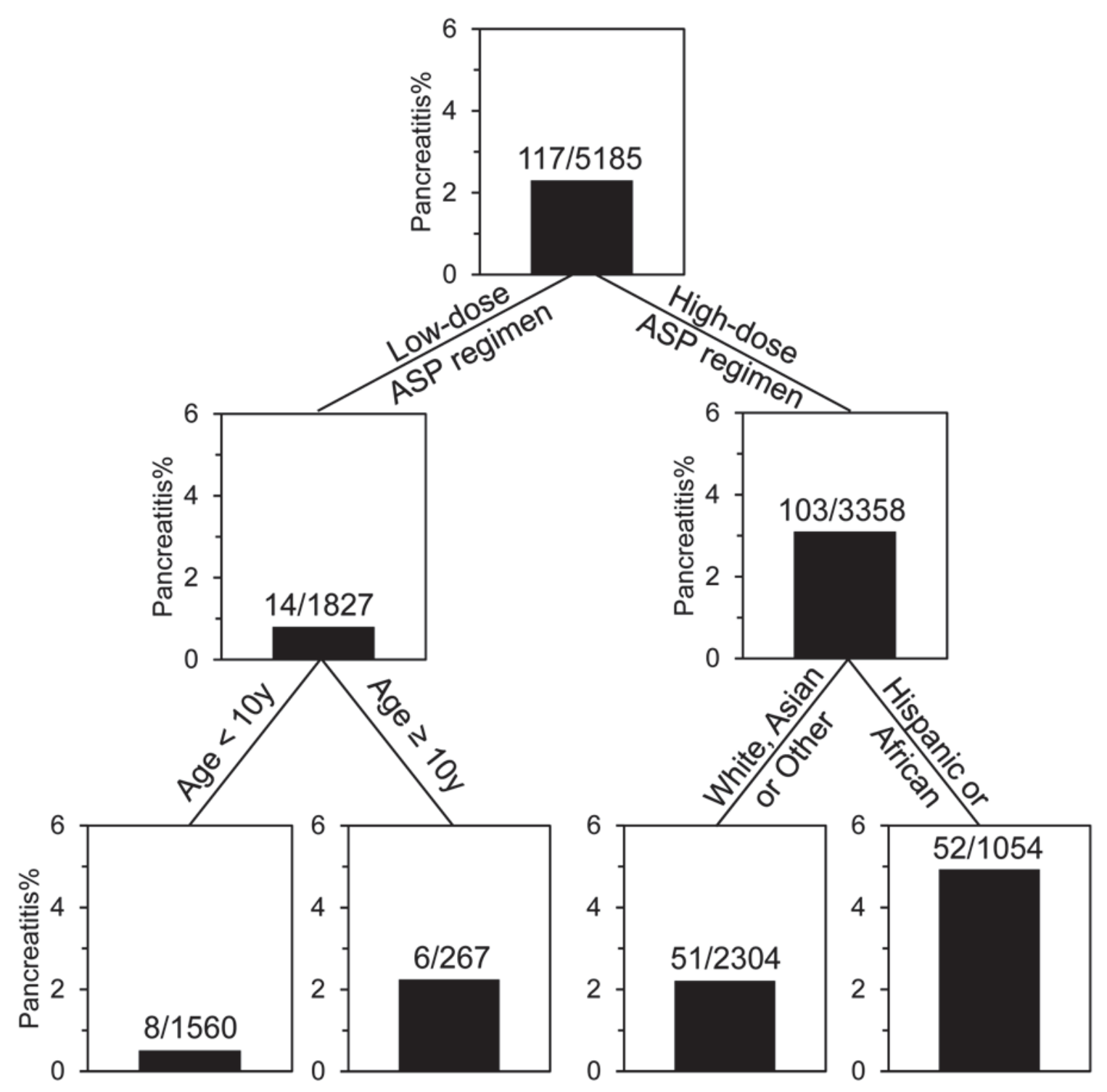

Figure 4-7. CART analysis of clinical risk factors for pancreatitis in the cohort (n = 5185)

The covariates included in the model are ancestry, age, lineage, gender, asparaginase regimen and formulation. 
Table 4-5. Six genes associated with pancreatitis from the gene-level analysis of nonsense SNPs

\begin{tabular}{|c|c|c|c|c|c|c|c|c|c|c|c|c|c|}
\hline \multirow[b]{2}{*}{ Gene } & \multirow[b]{2}{*}{ Chr } & \multirow[b]{2}{*}{$\begin{array}{l}\text { Nonsense } \\
\text { SNPs } \\
\text { (major } \\
\text { allele/risk } \\
\text { allele) }\end{array}$} & \multirow[b]{2}{*}{$\begin{array}{l}\text { Population } \\
\text { RAF\% } \\
\text { (n) }\end{array}$} & \multirow[b]{2}{*}{$\begin{array}{l}\text { CADD } \\
\text { score }^{b}\end{array}$} & \multicolumn{4}{|c|}{ Cohort $(n=3256)$} & \multicolumn{4}{|c|}{ Case-control $(n=213)$} & \multirow[b]{2}{*}{$\begin{array}{c}\text { PPV/ } \\
\text { NPV } \\
(\mathbf{n}= \\
\text { 3256) }\end{array}$} \\
\hline & & & & & $\begin{array}{l}\text { RAF\% } \\
\text { in } \\
\text { patients } \\
\text { with AP } \\
(\mathrm{n}=95)\end{array}$ & $\begin{array}{l}\text { RAF\% in } \\
\text { patients } \\
\text { without } \\
\text { AP } \\
(n=\mathbf{3 1 6 1}) \\
\end{array}$ & $\begin{array}{c}\text { Coeff- } \\
\text { icient }^{c} \\
(\mathrm{SE})\end{array}$ & $P^{\mathbf{c}}$ & $\begin{array}{c}\mathbf{R A F} \% \\
\text { in } \\
\text { cases } \\
(n=71)\end{array}$ & $\begin{array}{l}\text { RAF\% } \\
\text { in } \\
\text { controls } \\
(n=142)\end{array}$ & $\begin{array}{l}\text { Coeff- } \\
\text { icient }^{d} \\
(\mathrm{SE})\end{array}$ & $P^{\mathrm{d}}$ & \\
\hline$C P A 2$ & 7 & $\begin{array}{c}\text { rs199695765 } \\
(\mathrm{C} / \mathrm{T})\end{array}$ & 0.009 (11) & 18.64 & 0.5 & 0 & $\begin{array}{c}6.5 \\
(1.1)\end{array}$ & $2.1 \times 10^{-9}$ & 0.8 & 0 & $\begin{array}{l}15.8 \\
(7.4)\end{array}$ & 0.03 & $100.0 / 95.6$ \\
\hline SERPINA9 & 14 & $\begin{array}{l}\text { rs78283108 } \\
(\mathrm{G} / \mathrm{A})\end{array}$ & $0.6(732)$ & 10.87 & 2.7 & 0.7 & $\begin{array}{c}1.2 \\
(0.4)\end{array}$ & 0.006 & 0.8 & 0 & $\begin{array}{l}16.2 \\
(6.7)\end{array}$ & 0.02 & $11.5 / 95.6$ \\
\hline \multirow[t]{2}{*}{$H S D 17 B 2$} & 16 & $\begin{array}{l}\text { rs8191246 } \\
(\mathrm{A} / \mathrm{G})\end{array}$ & $1.4(1557)$ & 6.948 & 3.3 & 0.7 & $\begin{array}{l}1.5 \\
(0.5)\end{array}$ & 0.006 & 3.4 & 0 & $\begin{array}{l}17.1 \\
(5.9)\end{array}$ & 0.004 & $17.2 / 95.7$ \\
\hline & & $\begin{array}{c}\text { rs200540657 } \\
(\mathrm{T} / \mathrm{G})\end{array}$ & $0.001(1)$ & 15.63 & 0.1 & 0 & & & 0 & 0 & & & $0.0 / 94.8$ \\
\hline$G C K R$ & 2 & $\begin{array}{c}\text { rs } 146053779 \\
(\mathrm{C} / \mathrm{T})\end{array}$ & 0.09 (111) & 14.41 & 1.1 & 0.3 & $\begin{array}{l}1.7 \\
(0.7)\end{array}$ & 0.015 & 0.8 & 0 & $\begin{array}{l}15.4 \\
(8.5)\end{array}$ & 0.07 & $16.7 / 95.6$ \\
\hline \multirow[t]{2}{*}{ ZNF233 } & 19 & $\begin{array}{l}\text { rs } 144014948 \\
\text { (G/A) }\end{array}$ & 0.09 (112) & 26.8 & 0.5 & 0.1 & $\begin{array}{l}1.9 \\
(0.8)\end{array}$ & 0.019 & 0.8 & 0 & $\begin{array}{l}15.5 \\
(8.3)\end{array}$ & 0.06 & $25.0 / 95.6$ \\
\hline & & $\begin{array}{c}\text { rs } 148348903 \\
(\mathrm{~T} / \mathrm{A})\end{array}$ & 0.09 (114) & 34.0 & 1.1 & 0.1 & & & 0 & 0 & & & $20.0 / 95.6$ \\
\hline \multirow[t]{2}{*}{$S L C 6 A 18$} & 5 & $\begin{array}{l}\text { rs } 143864547 \\
\text { (C/A) }\end{array}$ & $0.003(4)$ & 15.71 & 0.5 & 0.02 & $\begin{array}{c}5.0 \\
(2.3)\end{array}$ & 0.028 & 0 & 0 & $\begin{array}{l}16.0 \\
(7.4)\end{array}$ & 0.03 & $50.0 / 95.6$ \\
\hline & & $\begin{array}{c}\mathrm{rs} 200802505 \\
(\mathrm{C} / \mathrm{T})\end{array}$ & $0.03(41)$ & 16.64 & 0 & 0.08 & & & 0.8 & 0 & & & $20.0 / 95.5$ \\
\hline
\end{tabular}




\section{Table 4-5. (Continued)}

The genes were rank ordered by SKAT P value in the cohort study, and then filtered by the direction in the case-control study. Only patients with exome array data are included in this analysis of nonsense SNPs. ${ }^{a}$ Population risk allele frequency and number of carriers of the nonsense SNP based on the ExAC data set ${ }^{166}$ of 61486 unrelated individuals of European, African, Latino and Asian origins. ${ }^{b} \mathrm{CADD}$ score ${ }^{160}$ was estimated for each nonsense SNP. The average CADD score of 9 SNPs is 17.7 (scores higher than 15 are considered as deleterious). ${ }^{\circ}$ Coefficient, standard error (SE) and SKAT P value were estimated using Cox proportional hazard model after collapsing all nonsense SNPs. ${ }^{\mathrm{d} C o e f f i c i e n t, ~ s t a n d a r d ~ e r r o r ~ a n d ~ S K A T ~ P ~ v a l u e ~ w e r e ~}$ estimated using logistic regression model after collapsing all nonsense SNPs. ${ }^{\mathrm{e} P P V}$ (positive predictive value) $=$ (number of patients who carried the nonsense SNP and developed pancreatitis)/(number of patients who carried the nonsense SNP). NPV (negative predictive value) $=$ (number of patients who did not carry the nonsense SNP or develop pancreatitis)/(number of patients who did not carry the nonsense SNP). The number of patients from the cohort and the case-control group were combined $(\mathrm{n}=3469)$. Chr, chromosome. AP, acute pancreatitis. RAF, risk allele frequency. 

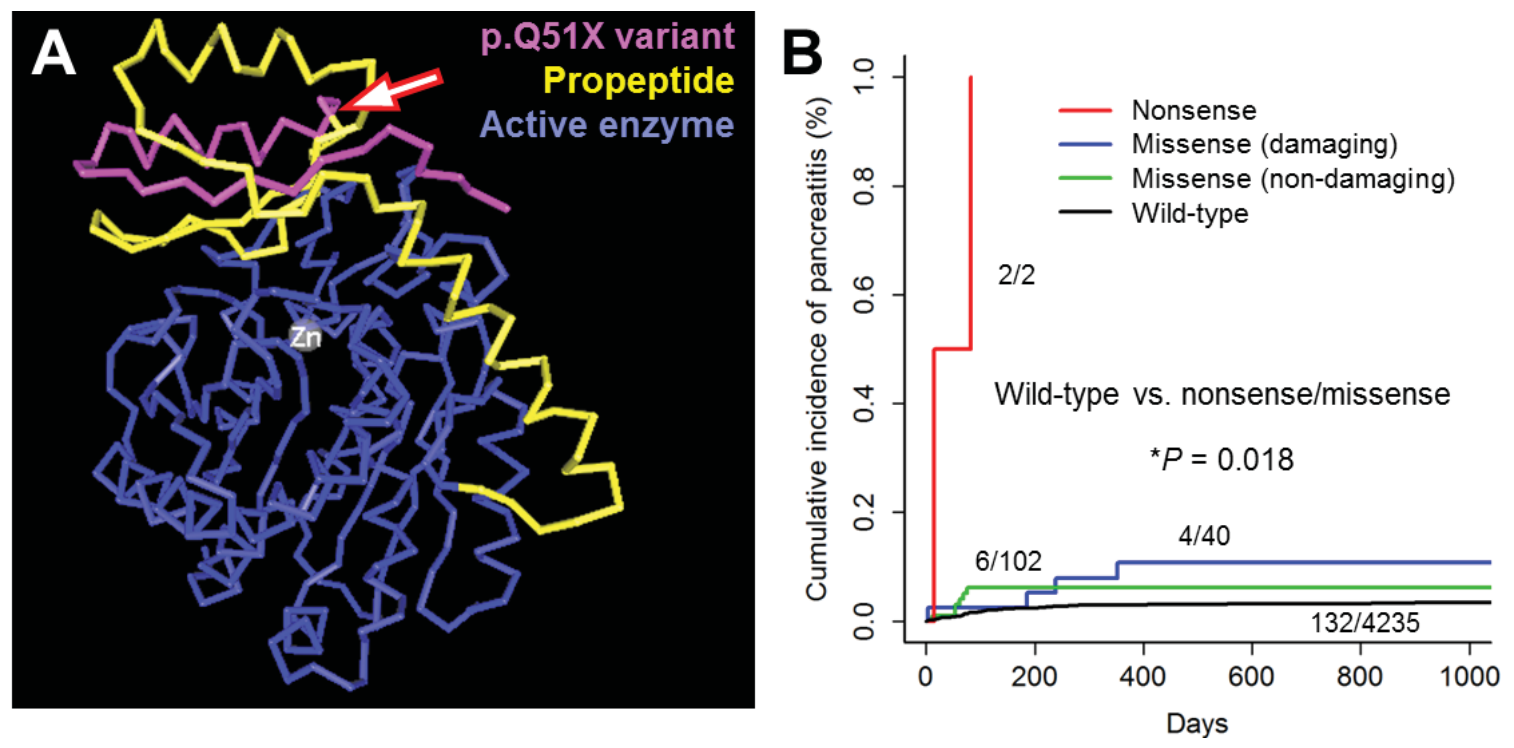

Figure 4-8. Nonsense SNP rs199695765 in $C P A 2$ associated with pancreatitis (A) Protein structure of proCPA2 (without the 16-amino-acid signal peptide) with the nonsense SNP rs199695765 (arrow) located in the propeptide domain. The unaffected region of p.Q51X variant (magenta), the affected propeptide (yellow) and the active enzyme moiety (blue) are shown. (B) Cumulative incidence of pancreatitis in patients who carried any CPA2 nonsense or missense SNPs (variant) was higher than that in patients who are classified as "wild-type," i.e. they carried none, or only synonymous or non-coding SNPs ( $* P=0.018$ after adjusting for clinical characteristics). 
Table 4-6. Top 20 common SNPs (cohort risk allele frequency $>1 \%$ ) significantly associated with pancreatitis in the cohort

\begin{tabular}{|c|c|c|c|c|c|c|c|c|c|c|c|c|c|c|}
\hline \multirow[b]{2}{*}{ SNP } & \multirow[b]{2}{*}{ Gene } & \multirow[b]{2}{*}{ Chr } & \multirow[b]{2}{*}{ Location $^{\mathrm{a}}$} & \multirow[b]{2}{*}{$\begin{array}{c}\text { Major } \\
\text { allele/ } \\
\text { risk } \\
\text { allele } \\
\end{array}$} & \multicolumn{4}{|c|}{$\begin{array}{c}\text { Cohort } \\
(\mathbf{n}=\mathbf{5 1 8 5})\end{array}$} & \multicolumn{4}{|c|}{$\begin{array}{l}\text { Case-control } \\
(\mathrm{n}=\mathbf{2 1 3})\end{array}$} & \multicolumn{2}{|c|}{$\begin{array}{c}\text { Cohort RAF vs race } \\
\quad(n=5185)\end{array}$} \\
\hline & & & & & $\begin{array}{c}\text { RAF\% in } \\
\text { patients } \\
\text { with AP } \\
(\mathrm{n}=117)\end{array}$ & $\begin{array}{c}\text { RAF\% in } \\
\text { patients } \\
\text { without AP } \\
(\mathrm{n}=\mathbf{5 0 6 8})\end{array}$ & $\begin{array}{c}\text { Coeff- } \\
\text { icient }^{b} \\
\text { (SE) }\end{array}$ & $P^{\mathrm{b}}$ & $\begin{array}{l}\text { RAF\% } \\
\text { in cases } \\
(n=71)\end{array}$ & $\begin{array}{l}\text { RAF\% in } \\
\text { controls } \\
(n=142)\end{array}$ & $\begin{array}{c}\text { Coeff- } \\
\text { icient }^{\mathrm{c}} \\
\text { (SE) }\end{array}$ & $P^{c}$ & $\begin{array}{c}\text { Hispanic } \\
\quad+\text { Black } \\
(\mathbf{n}=\mathbf{1 5 2 7})\end{array}$ & $\begin{array}{c}\text { White } \\
\text { +Asian } \\
(\mathrm{n}=\mathbf{3 1 6 8})\end{array}$ \\
\hline rs9849262 & FHIT & 3 & 60937049 & $\mathrm{C} / \mathrm{T}$ & 4.7 & 1.0 & $1.4(0.3)$ & $8.4 \times 10^{-6}$ & 0.7 & 0 & $15(11.2)$ & 0.18 & $2.8^{*}$ & $0.2^{*}$ \\
\hline rs12589386 & & 14 & 47223965 & $\mathrm{~A} / \mathrm{G}$ & 8.5 & 3.7 & $1.1(0.2)$ & $1.8 \times 10^{-5}$ & 9.4 & 3.2 & $1.0(0.4)$ & 0.03 & 2.2 & 3.9 \\
\hline rs 17377657 & & 8 & 78054203 & $\mathrm{C} / \mathrm{T}$ & 11.5 & 5.9 & $0.9(0.2)$ & $1.8 \times 10^{-5}$ & 6.5 & 2.9 & $1.0(0.5)$ & 0.06 & 3.0 & 7.3 \\
\hline rs7826058 & DOCK5 & 8 & 25095058 & $\mathrm{~A} / \mathrm{C}$ & 8.1 & 2.8 & $1.1(0.3)$ & $2.7 \times 10^{-5}$ & 5.1 & 1.1 & $1.2(0.7)$ & 0.07 & $5.7^{*}$ & $1.3^{*}$ \\
\hline rs1768056 & ACTN2 & 1 & 236890781 & $\mathrm{G} / \mathrm{A}$ & 13.2 & 3.7 & $1.0(0.3)$ & $4.3 \times 10^{-5}$ & 8.0 & 2.9 & $0.8(0.6)$ & 0.13 & $11.2^{*}$ & $0.6^{*}$ \\
\hline rs 16955095 & ITFGI & 16 & 47333623 & $\mathrm{C} / \mathrm{A}$ & 11.1 & 4.7 & $0.9(0.2)$ & $4.6 \times 10^{-5}$ & 3.6 & 2.5 & $0.3(0.6)$ & 0.56 & $6.1 *$ & $4.0^{*}$ \\
\hline rs 17117423 & & 14 & 47008512 & $\mathrm{~T} / \mathrm{C}$ & 9.1 & 4.2 & $1.0(0.2)$ & $5.0 \times 10^{-5}$ & 8.7 & 2.9 & $1.2(0.5)$ & 0.02 & 3.3 & 4.3 \\
\hline rs 16892294 & AGPAT4 & 46 & 161626177 & $\mathrm{C} / \mathrm{T}$ & 29.1 & 13.1 & $0.7(0.2)$ & $1.0 \times 10^{-4}$ & 23.2 & 12.9 & $0.5(0.3)$ & 0.08 & $28.2^{*}$ & $6.3^{*}$ \\
\hline rs9859201 & FHIT & 3 & 60935372 & $\mathrm{~T} / \mathrm{A}$ & 4.3 & 1.1 & $1.3(0.3)$ & $1.2 \times 10^{-4}$ & 0.7 & 0 & $15(11.2)$ & 0.18 & $3.0^{*}$ & $0.2^{*}$ \\
\hline rs1034347 & & 21 & 21535391 & $\mathrm{G} / \mathrm{A}$ & 17.1 & 10.4 & $0.7(0.2)$ & $1.6 \times 10^{-4}$ & 13.0 & 9.0 & $0.4(0.4)$ & 0.24 & $11.7^{*}$ & $9.9^{*}$ \\
\hline rs16953067 & ITFGI & 16 & 47200165 & $\mathrm{~A} / \mathrm{G}$ & 11.1 & 5.0 & $0.9(0.2)$ & $1.6 \times 10^{-4}$ & 3.6 & 2.9 & $0.2(0.6)$ & 0.71 & $6.7^{*}$ & $4.1^{*}$ \\
\hline rs 1564947 & MICAL2 & 11 & 12176409 & $\mathrm{G} / \mathrm{A}$ & 68.8 & 50.7 & $0.6(0.2)$ & $1.8 \times 10^{-4}$ & 58.0 & 42.1 & $0.5(0.2)$ & 0.06 & $71.1^{*}$ & $40.2^{*}$ \\
\hline rs11961305 & AGPAT4 & 46 & 161627211 & $\mathrm{G} / \mathrm{A}$ & 31.2 & 14.9 & $0.6(0.2)$ & $1.8 \times 10^{-4}$ & 23.9 & 15.5 & $0.4(0.3)$ & 0.25 & $32.3^{*}$ & $6.6^{*}$ \\
\hline rs16907254 & & 11 & 4931083 & $\mathrm{C} / \mathrm{T}$ & 24.1 & 13.0 & $0.6(0.2)$ & $1.8 \times 10^{-4}$ & 13.8 & 9.0 & $0.4(0.3)$ & 0.28 & $18.3^{*}$ & $11.1^{*}$ \\
\hline rs11606424 & & 11 & 43159756 & $\mathrm{C} / \mathrm{A}$ & 11.6 & 5.4 & $0.8(0.2)$ & $1.9 \times 10^{-4}$ & 8.0 & 5.8 & $0.2(0.4)$ & 0.65 & 6.1 & 5.3 \\
\hline rs 2010463 & MICAL2 & 11 & 12186296 & $\mathrm{C} / \mathrm{T}$ & 61.1 & 55.3 & $0.6(0.2)$ & $2.0 \times 10^{-4}$ & 54.5 & 34.6 & $0.7(0.2)$ & 0.003 & $56.4^{*}$ & $39.2^{*}$ \\
\hline rs1341861 & ACTN2 & 1 & 236883686 & $\mathrm{~T} / \mathrm{G}$ & 16.7 & 6.2 & $0.8(0.2)$ & $2.6 \times 10^{-4}$ & 9.4 & 3.6 & $0.8(0.5)$ & 0.10 & $17.2^{*}$ & $1.2^{*}$ \\
\hline rs 16945382 & $P H K B$ & 16 & 47521171 & $\mathrm{~T} / \mathrm{G}$ & 12.0 & 5.6 & $0.8(0.2)$ & $2.7 \times 10^{-4}$ & 6.9 & 5.9 & $1.0(0.5)$ & 0.06 & $8.5^{*}$ & $4.0^{*}$ \\
\hline rs 17053225 & DOCK 5 & 8 & 25097310 & $\mathrm{~A} / \mathrm{G}$ & 10.6 & 4.5 & $1.0(0.3)$ & $2.8 \times 10^{-4}$ & 5.9 & 0.7 & $1.7(0.7)$ & 0.02 & $10.1^{*}$ & $1.6^{*}$ \\
\hline rs 10831758 & MICAL2 & 11 & 12218497 & $\mathrm{C} / \mathrm{T}$ & 39.7 & 25.8 & $0.5(0.2)$ & $3.0 \times 10^{-4}$ & 31.2 & 21.9 & $0.4(0.3)$ & 0.11 & $36.5^{*}$ & $20.3^{*}$ \\
\hline
\end{tabular}




\section{Table 4-6. (Continued)}

The SNPs were rank ordered by $\mathrm{P}$ value in the cohort study, and then filtered by the direction in the case-control study. The RAFs of combined race groups (Hispanic + Black and White + Asian) in the cohort $(\mathrm{n}=5185)$ are shown. ${ }^{*}$ Higher RAF $(P<$ 0.05) in the Hispanic and black groups than in whites and Asians. ${ }^{a}$ Physical location of SNP based on Feb 2009 human genome assembly (hg19). ${ }^{\mathrm{b}}$ Coefficient, standard error and P value were estimated using Cox proportional hazard model. ${ }^{\mathrm{c}}$ Coefficient, standard error and $\mathrm{P}$ value were estimated using logistic regression model. Chr, chromosome. AP, acute pancreatitis. RAF, risk allele frequency. 


\section{Deep sequencing detected novel variants in $C P A 2$ and other candidate genes}

We identified additional variants by sequencing 283 genes, 42 of which were $a$ priori classified as likely candidate genes $(n=34)$ for pancreatitis based on our GWAS results or based on prior links to pancreatitis in other settings ( $\mathrm{n}=8 ;$ PRSS1, PRSS2, SPINK1, CFTR, CASR, CTRC, CPA1, CLDN2; Table 4-3).

For the 4217 children in the cohort and 162 in the case-control group with available deep sequencing data, 380 variants were identified in CPA2, including 354 variants that were not interrogated on either the exome or SNP 6.0 array (see Appendix C, Table C-4). Sixteen $C P A 2$ SNPs were associated $(P<0.05)$ with pancreatitis, and 13 of 24 patients who carried at least one of these variants developed pancreatitis.

Interestingly, two of three patients who had multiple (i.e. three) episodes carried one of these CPA2 variants (the nonsense SNP rs199695765 and an intronic SNP chr7:129915817 G>A). Patients who carried CPA2 coding variants had a higher risk of pancreatitis than those who did not carry any coding variants $(\mathrm{OR}=2.4,95 \% \mathrm{CI} 1.2-5.1$, $P=0.018$ ) after adjusting for clinical features (Figure 4-8B). For patients who carried the nonsense SNP rs199695765, the estimated risk was uncertain but very high $(\mathrm{OR}=$ $587,95 \%$ CI 66.8-5166, $P=9.0 \times 10^{-9}$ ). The positive predictive value (PPV) and negative predictive value (NPV) of rs199695765 were 100\% (95\% CI: $19.3-100 \%)$ and 95.6\% (95\% CI: 94.8 - 96.2\%). Of 61486 subjects reported in the Exome Aggregation Consortium (ExAC) data set, ${ }^{166}$ a total of 11 individuals were heterozygous for this variant, yielding an allele frequency in the general population of only $0.009 \%$ (Table 4-5).

Similar to the initial GWAS, we conducted a gene-level analysis of nonsense variants in the 42 putative pancreatitis genes versus the 241 non-pancreatitis genes (see Appendix C, Table C-5). Among the 283 genes, 21 were related to pancreatitis at the $P$ $<0.05$ level, 16 of which were pancreatitis genes. Based on sequencing, a higher proportion of putative candidate pancreatitis genes had significant association than the non-candidate genes at $P<0.05\left(16 / 42\right.$ vs $\left.5 / 241, P=2.7 \times 10^{-11}\right)$. The pathway analysis showed that the top networks associated with the 42 genes included endocrine system disorders, cell cycle and proliferation, and gastrointestinal disease (Figure C-2). When the analysis was limited to the 34 genes identified by initial GWAS (Table 4-3), these pathways still remained the most significant (Figure C-3).

In the gene-level analysis, HOGA1 with a nonsense SNP (chr10:99361676 C $>\mathrm{T}$, p.R255X) showed the strongest association in the cohort $\left(P=6.4 \times 10^{-11}\right)$. The only patient who was heterozygous for this nonsense variant developed pancreatitis very early, at day 17. $C P A 2$ was ranked second (for nonsense SNPs, $P=6.7 \times 10^{-11}$ ) and remained significant when the gene-based analysis included any missense SNPs $\left(P=5.5 \times 10^{-3}\right)$ or any coding variants $(P=0.045)$.

Among the 8 candidate genes previously associated with pancreatitis in other settings, 1732 coding polymorphisms were identified via sequencing (Table C-6). Eighty-one SNPs were associated with pancreatitis at the $P<0.05$ level in either the 
cohort or the case-control group, but none of them were significant in both groups. A variant (chr7:117119516 G $>$ A) upstream of CFTR showed the strongest association in the cohort $\left(P=4.4 \times 10^{-9}\right)$; this variant was carried by only one patient, who developed early pancreatitis at day 25 . None of the candidate genes were significantly associated $(P$ $<0.05$ ) with pancreatitis in the gene-level analysis.

\section{Discussion}

Acute pancreatitis has long been recognized as a serious complication of asparaginase-containing regimens and can limit the ability to deliver chemotherapy. However, the pathogenesis and mechanisms remain unknown, and it is not currently possible predict whether and when a patient will experience this toxicity. There is no definitive treatment for acute pancreatitis; only supportive care is given. ${ }^{61}$ Thus, the genetic and non-genetic risk factors we discovered in this largest study to date may have importance for understanding the mechanisms and developing targeted interventions for patients with predisposition.

Here we report that variants in $C P A 2$, encoding carboxypeptidase $\mathrm{A} 2$, were associated with risk of acute pancreatitis in patients with ALL. This gene encodes a 417amino-acid proenzyme (proCPA2) that comprises a 16-amino-acid signal sequence, a 94amino-acid propeptide and an active enzyme moiety. ${ }^{167}$ There are 11 exons; the nonsense SNP rs199695765 is in exon 2, resulting in an early termination in the propeptide region (Figure 4-8A). Among 4379 patients tested, the two who carried this variant developed severe pancreatitis within weeks of their first dose of the drug (Figure 4-8B), suggesting that asparaginase will not be tolerated long enough to be effective in these rare patients. Whether the MAF $(0.023 \%)$ in this ALL cohort was comparable to or higher than the MAF in the general population $(0.009 \%)$ is not clear, but in any case, it is rare. In addition to rs 199695765 , there were 15 other variants in CPA2 associated with pancreatitis (Table C-4). The 16 variants were carried by 25 of 4379 patients (collective $\mathrm{MAF}=0.6 \%$ ) with sequencing data, and all 10 patients who carried top $10 C P A 2$ variants developed pancreatitis. These data suggest that $C P A 2$ variant carriers may be at unacceptably high risk of pancreatitis if challenged with asparaginase. ALL treatment regimens vary substantially in the amount of asparaginase they include, and many "low asparaginase" regimens achieve comparable outcomes to "high asparaginase" regimens. Selecting an ALL treatment regimen to exclude asparaginase may be preferable for these rare individuals.

The underlying mechanism by which $C P A 2$ variants confer a higher risk of acute pancreatitis is unclear. Pancreatic carboxypeptidases are zinc metalloenzymes that hydrolyze C-terminal amino acids. Two types of carboxypeptidases, A-type (CPA) and B-type (CPB) are both secreted from pancreatic acinar cells as pro-enzymes, then cleaved and activated by trypsin in the duodenum. There are two isoforms of CPA in humans, CPA1 and CPA2, which have significant similarity in amino acid sequence and threedimensional structure. ${ }^{168}$ Interestingly, patients heterozygous for functionally impaired variants in a related isoform, $C P A 1$, are at higher risk of non-alcoholic chronic 
pancreatitis. ${ }^{80}$ It was suggested that the mechanisms may involve misfolding-induced endopasmic reticulum stress in pancreatic acinar cells, rather than premature activation of trypsin. ${ }^{80}$ It is possible that similar mechanisms may underlie how CPA2 variants predispose to acute pancreatitis.

The top ranked gene based on sequencing of 283 pharmacogenomic candidate genes was $H O G A 1$, an aldolase that catalyzes the final step in the metabolic pathway of hydroxyproline, releasing glyoxylate and pyruvate. ${ }^{169}$ Only one patient of 4379 studied carried the nonsense variant chr10:99361676 C $>$ T (R255X). Reduced enzymatic activity of HOGA1 may cause calcium oxalate urolithiasis; ${ }^{170}$ perhaps a similar cytosolic excess of oxalate could lead to pancreatitis, as pancreas is a potential site of hydroxyproline degradation. $^{171}$

Whereas variants in $C P A 2$ and $H O G A 1$ were rare, the genome-wide analysis of common variants (Table 4-6) also provided some insights into other possible predisposing mechanisms. The lead gene FHIT (fragile histidine triad protein) is involved in purine metabolism. ${ }^{169}$ Because thiopurines are essential to ALL therapy and can also induce acute pancreatitis, ${ }^{150,151}$ this finding may link thiopurines to some cases of pancreatitis in our study.

Other top genes (DOCK5, ACTN2 and MICAL2) clustered in cytoskeleton function, supporting the hypothesis that the cytoskeleton may be involved in the pathogenesis of pancreatitis. ${ }^{172} 173$ Interruption of cytoskeleton and pancreatic enzyme secretion has been seen in animal models of drug-induced pancreatitis ${ }^{172}$ and in patients with acute pancreatitis. ${ }^{174}$ It has been well established that exocytosis highly depends on vesicle trafficking via cytoskeleton in all types of secretory cells including pancreatic acinar cells. ${ }^{175,176}$ There were also case reports of clinical pancreatitis following cytoskeleton inhibitors such as brentuximab vedotin ${ }^{177}$ and taxanes. ${ }^{178}$ Indeed cerulein, used as the primary chemical tool to initiate pancreatitis in animal models, causes cytoskeleton disorganization. ${ }^{179}$ DOCK5 (dedicator of cytokinesis 5) encodes a guanine nucleotide exchange (GEF) factor that activates small GTPases including Rho and Rac, which are key regulators of actin distribution and amylase secretion in pancreatic acinar cells. ${ }^{180,181}$ ACTN2 (actinin-2) encodes an actin-binding protein that bundles actin filaments in multiple cell types and cytoskeleton frameworks. ${ }^{182}$ It has been reported that $\alpha$-actinin played a putative role in regulated exocytosis by connecting the actin filaments to secretory vesicles. ${ }^{183}$ Intriguingly, the interaction between actin and actinin can be affected by increased level of intracellular $\mathrm{Ca}^{2+}$, which was also observed concurrently with secretion blockade has been implicated in the early pathogenesis of acute pancreatitis. MICAL2 (molecule interacting with CasL protein 2) encodes a member of MICAL family that interacts with small GTPase Rab1, which plays a role in vesicle trafficking from the endoplasmic reticulum to the Golgi. ${ }^{184}$ MICAL proteins also bind to actin, regulate cytoskeletal rearrangements and play important regulatory functions in membrane trafficking. ${ }^{185}$ In addition, among the 42 genes that were putatively associated with pancreatitis or ranked the top in the GWAS (Table 4-6), 10 (24\%) have been shown to be involved in cytoskeleton regulation and vesicle transportation. Together, these findings suggest that the addition to a role for highly penetrant rare genetic variants in 
some cases, common variants affecting purines or cytoskeleton function may affect risk of pancreatitis.

Eight genes (e.g. PRSS1, PRSS2, SPINK1, CTRC, CFTR, CASR, CPA1, CLDN2) have been identified as risk factors for non-drug-induced pancreatitis. Most SNPs in these genes were not as strongly associated as the variants discovered using a genome-wide agnostic approach (Table C-6). Using gene-based approaches, none of these eight genes were among these with $P<0.05$, using deep sequencing data (Table C-5). HLA$D R B 1 * 07: 01$ (tagged by SNP rs17885382) has been associated with thiopurine-induced pancreatitis $^{78}$ but we found no association with pancreatitis in our study $(P=0.1)$. These findings suggest different underlying mechanisms of asparaginase-induced versus other forms of pancreatitis. The fact that asparaginase-induced pancreatitis was dose-dependent (Figure 4-3), whereas thiopurine-induced pancreatitis was not, ${ }^{78}$ also suggests different pathogenesis of pancreatitis caused by these two important antileukemic drugs.

Consistent with reports from other groups, ${ }^{47-49,65,186}$ older age was associated with higher risk of pancreatitis. The relationship was also observed in children who had pancreatitis with other etiologies, e.g. biliary, traumatic, hereditary or idiopathic pancreatitis, ${ }^{187-189}$ indicating an intrinsic pancreatic susceptibility that increases with age. It has been shown that serum amylase levels were positively associated with age in healthy children ${ }^{190}$ and those who developed acute pancreatitis. ${ }^{191}$

Similar to previous studies, ${ }^{48,50}$ gender and ALL lineage did not seem to influence the risk of pancreatitis in this study. We observed similar pancreatitis rates using two different asparaginase formulations, which is consistent with some studies, ${ }^{27,49}$ but different from several earlier ones ${ }^{40,47,65}$ that PEG-asparaginase induced more pancreatitis than native E.coli asparaginase did. However, in those studies ${ }^{40,47,65}$ native $E$. coli-asparaginase was usually given at a lower dose (e.g. $6000 \mathrm{U} / \mathrm{m}^{2}$ thrice weekly) comparing to that used in current practice $\left(10000 \mathrm{U} / \mathrm{m}^{2}\right.$ thrice weekly or $25000 \mathrm{U} / \mathrm{m}^{2}$ weekly) so the patients on native $E$. coli arm might have been exposed to less asparaginase than the equivalent dose given to those on PEG-asparaginase arm (typically at $2500 \mathrm{U} / \mathrm{m}^{2}$ biweekly).

Although other agents (e.g. glucocorticoids and thiopurines) can cause pancreatitis, asparaginase is mostly likely the cause in most of our patients, due to its early onset (coinciding with asparaginase; Figure 4-4), and its relationship to asparaginase cumulative dose and duration ${ }^{49,50,52,53}$ (Figure 4-3B and C). The association with genetically defined ancestry is consistent with an important contribution of genetics in the development of pancreatitis (Table 4-4, Figures 4-6 and 4-7); for example, risk alleles for 16 of the top 20 common variants (Table 4-6) were present at a higher frequency (at $P<0.05$ ) in the Hispanic and black groups than in whites and Asians. Notably, of the two carriers of the CPA2 nonsense SNP at rs199695765, one was of European and one had high Native American ancestry. 


\section{Conclusion}

In summary, this comprehensive study showed that older age, higher exposure to asparaginase, higher Native American ancestry or African ancestry were independent risk factors for pancreatitis in children and young adults treated for ALL. Rare

nonsynonymous variants in $C P A 2$, especially a nonsense variant rs 199695765 , were highly penetrant in their effect on risk of pancreatitis, and more common variants in genes critical to purine metabolism and cytoskeleton function were also associated with development of pancreatitis. Our findings are consistent with a mixed genetic architecture underlying serious adverse drug effects, wherein a combination of rare but highly penetrant and common but weakly penetrant genetic risk factors contribute to genetic risk. For the small number of patients carrying the highly penetrant $C P A 2$ nonsense variants, consideration should be given to treatment with a non-asparaginase containing ALL chemotherapy regimens. 


\section{CHAPTER 5. SUMMARY}

In this study, we have discussed the major adverse effects of asparaginase in patients with pediatric ALL, including hypersensitivity, acute pancreatitis, and the potentiating effect on osteonecrosis.

Hypersensitivity is the most common dose-limiting event of asparaginase. We showed that measurements of serum antibodies against Elspar may be useful in clinical practice. Higher antibody levels were associated with higher risk of clinical allergy, and a greater attenuation of serum asparaginase activity. Osteonecrosis, an adverse event associated with glucocorticoids, was associated with lower anti-Elspar antibody levels (i.e. higher serum asparaginase activity).

Thus, we studied the effect of asparaginase on glucocorticoid-induced osteonecrosis using a murine model. We were able to recapitulate the clinical observation that asparaginase treatment added to dexamethasone was associated with more arteriopathy and osteonecrosis in mice than dexamethasone treatment alone. The drug interaction confirmed in this preclinical study may be clinically important, as glucocorticoids and asparaginase are concomitantly used in the treatment ALL.

Acute pancreatitis is one of the serious adverse effects that may cause temporary or permanent withdrawal of asparaginase treatment. However, only a few large studies focused on clinical risk factors of pancreatitis, and there was previously no study of genetic risk factors. Here we performed a comprehensive study using a large cohort of pediatric patient with ALL and an independent case-control group. Age, asparaginase exposure, Native American ancestry or African ancestry were independent clinical risk factors. Rare nonsynonymous variants in $C P A 2$, especially a nonsense variant rs199695765, were highly penetrant genetic risk factors. These findings may have implications for modifying therapy decisions in the future.

In summary, our studies have identified risk factors for adverse effects of asparaginase, yielded insight into the underlying mechanisms of drug response, and provided important guidance for precision medicine. Future work in our laboratory that builds on my work will include evaluating diagnostic tests to predict allergy to Oncaspar (the PEGylated form), further analyses of the mechanism by which Oncaspar contributes to osteonecrosis, and creation of transgenic mice bearing the CPA2 variant to test for effects on pancreatitis preclinically. 


\section{LIST OF REFERENCES}

1. Carroll WL, Bhojwani D, Min DJ, et al. Pediatric acute lymphoblastic leukemia. Hematology.(Am.Soc.Hematol.Educ.Program.). 2003:102-131.

2. Calvelli T, Denny TN, Paxton H, Gelman R, Kagan J. Guideline for flow cytometric immunophenotyping: a report from the National Institute of Allergy and Infectious Diseases, Division of AIDS. Cytometry. Oct 1993;14(7):702-715.

3. Pui CH, Robison LL, Look AT. Acute lymphoblastic leukaemia. Lancet. Mar 22 2008;371(9617):1030-1043.

4. Ward E, DeSantis C, Robbins A, Kohler B, Jemal A. Childhood and adolescent cancer statistics, 2014. CA: a cancer journal for clinicians. Mar-Apr 2014;64(2):83-103.

5. Pui CH, Relling MV, Downing JR. Acute lymphoblastic leukemia. The New England journal of medicine. Apr 8 2004;350(15):1535-1548.

6. Mullighan CG. The molecular genetic makeup of acute lymphoblastic leukemia. Hematology / the Education Program of the American Society of Hematology. American Society of Hematology. Education Program. 2012;2012:389-396.

7. Pane F, Intrieri M, Quintarelli C, Izzo B, Muccioli GC, Salvatore F. BCR/ABL genes and leukemic phenotype: from molecular mechanisms to clinical correlations. Oncogene. 2002;21(56):8652-8667.

8. Pui CH, Campana D, Pei D, et al. Treating childhood acute lymphoblastic leukemia without cranial irradiation. The New England journal of medicine. Jun 25 2009;360(26):2730-2741.

9. Kidd JG. Regression of transplanted lymphomas induced in vivo by means of normal guinea pig serum. I. Course of transplanted cancers of various kinds in mice and rats given guinea pig serum, horse serum, or rabbit serum. The Journal of experimental medicine. Dec 1953;98(6):565-582.

10. Kidd JG. Regression of transplanted lymphomas induced in vivo by means of normal guinea pig serum. II. Studies on the nature of the active serum constituent: histological mechanism of the regression: tests for effects of guinea pig serum on lymphoma cells in vitro: discussion. The Journal of experimental medicine. Dec 1953;98(6):583-606.

11. Broome JD. Evidence that the L-asparaginase of guinea pig serum is responsible for its antilymphoma effects. II. Lymphoma 6C3HED cells cultured in a medium devoid of L-asparagine lose their susceptibility to the effects of guinea pig serum in vivo. The Journal of experimental medicine. Jul 1963;118:121-148.

12. Mashburn LT, Wriston JC, Jr. Tumor Inhibitory Effect of L-Asparaginase from Escherichia Coli. Archives of biochemistry and biophysics. May 1964;105:450452.

13. Dolowy WC, Henson D, Cornet J, Sellin H. Toxic and antineoplastic effects of Lasparaginase. Study of mice with lymphoma and normal monkeys and report on a child with leukemia. Cancer. Dec 1966;19(12):1813-1819.

14. Roberts J, Burson G, Hill JM. New procedures for purification of L-asparaginase with high yield from Escherichia coli. Journal of bacteriology. Jun 1968;95(6):2117-2123. 
15. Su N, Pan YX, Zhou M, Harvey RC, Hunger SP, Kilberg MS. Correlation between asparaginase sensitivity and asparagine synthetase protein content, but not mRNA, in acute lymphoblastic leukemia cell lines. Pediatric blood \& cancer. Feb 2008;50(2):274-279.

16. Iwamoto S, Mihara K, Downing JR, Pui CH, Campana D. Mesenchymal cells regulate the response of acute lymphoblastic leukemia cells to asparaginase15. J.Clin.Invest. 2007;117(4):1049-1057.

17. Wriston JC, Jr., Yellin TO. L-asparaginase: a review. Advances in enzymology and related areas of molecular biology. 1973;39:185-248.

18. Muller HJ, Boos J. Use of L-asparaginase in childhood ALL. Critical reviews in oncology/hematology. Aug 1998;28(2):97-113.

19. Capizzi RL, Bertino JR, Handschumacher RE. L-asparaginase. Annual review of medicine. 1970;21:433-444.

20. Riccardi R, Holcenberg JS, Glaubiger DL, Wood JH, Poplack DG. Lasparaginase pharmacokinetics and asparagine levels in cerebrospinal fluid of rhesus monkeys and humans. Cancer research. 1981;41:4554-4558.

21. Kawedia JD, Liu C, Pei D, et al. Dexamethasone exposure and asparaginase antibodies affect relapse risk in acute lymphoblastic leukemia. Blood. Feb 16 2012;119(7):1658-1664.

22. Uren JR, Ragin RC. Improvement in the therapeutic, immunological, and clearance properties of Escherichia coli and Erwinia carotovora L-asparaginases by attachment of poly-DL-alanyl peptides. Cancer research. Jun 1979;39(6 Pt 1):1927-1933.

23. Dinndorf PA, Gootenberg J, Cohen MH, Keegan P, Pazdur R. FDA drug approval summary: pegaspargase (oncaspar) for the first-line treatment of children with acute lymphoblastic leukemia (ALL). The oncologist. Aug 2007;12(8):991-998.

24. Fernandez CA, Smith C, Yang WJ, et al. Genetic Variation in NFATC2 Is Associated with a Higher Risk of Asparaginase Allergy. Blood. Dec 6 2014;124(21).

25. Prescribing Information of ERWINAZE (asparaginase Erwinia chrysanthemi). U.S. Food and Drug Administration. 2014; http://www.accessdata.fda.gov/drugsatfda_docs/label/2011/125359lbl.pdf.

26. Boos J, Werber G, Ahlke E, et al. Monitoring of asparaginase activity and asparagine levels in children on different asparaginase preparations. European journal of cancer. Aug 1996;32A(9):1544-1550.

27. Duval M, Suciu S, Ferster A, et al. Comparison of Escherichia coli-asparaginase with Erwinia-asparaginase in the treatment of childhood lymphoid malignancies: results of a randomized European Organisation for Research and Treatment of Cancer-Children's Leukemia Group phase 3 trial. Blood. 2002;99(8):2734-2739.

28. Chien WW, Allas S, Rachinel N, et al. Pharmacology, immunogenicity, and efficacy of a novel pegylated recombinant Erwinia chrysanthemi-derived Lasparaginase. Investigational new drugs. Oct 2014;32(5):795-805.

29. Prescribing Information for ELSPAR. U.S. Food and Drug Administration. 2013. 
30. Yang L, Panetta JC, Cai X, et al. Asparaginase may influence dexamethasone pharmacokinetics in acute lymphoblastic leukemia. Journal of clinical oncology: official journal of the American Society of Clinical Oncology. 2008;26(12):19321939.

31. Woo MH, Hak LJ, Storm MC, et al. Hypersensitivity or development of antibodies to asparaginase does not impact treatment outcome of childhood acute lymphoblastic leukemia. Journal of clinical oncology : official journal of the American Society of Clinical Oncology. 2000;18(7):1525-1532.

32. Asselin BL, Whitin JC, Coppola DJ, Rupp IP, Sallan SE, Cohen HJ. Comparative pharmacokinetic studies of three asparaginase preparations. Journal of clinical oncology : official journal of the American Society of Clinical Oncology. 1993;11:1780-1786.

33. Killander D, Dohlwitz A, Engstedt L, et al. Hypersensitive reactions and antibody formation during L-asparaginase treatment of children and adults with acute leukemia. Cancer. -32676 1976;37:220-228.

34. Avramis VI, Avramis EV, Hunter W, Long MC. Immunogenicity of native or pegylated E. coli and Erwinia asparaginases assessed by ELISA and surface plasmon resonance (SPR-biacore) assays of IgG antibodies (Ab) in sera from patients with acute lymphoblastic leukemia (ALL). Anticancer research. Jan 2009;29(1):299-302.

35. Korholz D, Wahn U, Jurgens H, Wahn V. [Allergic reactions in treatment with Lasparaginase. Significance of specific IgE antibodies]. Monatsschrift Kinderheilkunde : Organ der Deutschen Gesellschaft fur Kinderheilkunde. Jan 1990;138(1):23-25.

36. Zalewska-Szewczyk B, Andrzejewski W, Mlynarski W, Jedrychowska-Danska K, Witas H, Bodalski J. The anti-asparagines antibodies correlate with L-asparagines activity and may affect clinical outcome of childhood acute lymphoblastic leukemia. Leukemia \& lymphoma. May 2007;48(5):931-936.

37. Cheung NK, Chau IY, Coccia PF. Antibody response to Escherichia coli Lasparaginase. Prognostic significance and clinical utility of antibody measurement. Am J Pediatr Hematol Oncol. Summer 1986;8(2):99-104.

38. Clarkson B, Krakoff I, Burchenal J, et al. Clinical results of treatment with E. coli L-asparaginase in adults with leukemia, lymphoma, and solid tumors. Cancer. Feb 1970;25(2):279-305.

39. Fabry U, Korholz D, Jurgens H, Gobel U, Wahn V. Anaphylaxis to Lasparaginase during treatment for acute lymphoblastic leukemia in children-evidence of a complement- mediated mechanism. Pediatr.Res. 1985;19:400-408.

40. Avramis VI, Sencer S, Periclou AP, et al. A randomized comparison of native Escherichia coli asparaginase and polyethylene glycol conjugated asparaginase for treatment of children with newly diagnosed standard-risk acute lymphoblastic leukemia: a Children's Cancer Group study. Blood. Mar 15 2002;99(6):19861994.

41. Armstrong JK, Hempel G, Koling S, et al. Antibody against poly(ethylene glycol) adversely affects PEG-asparaginase therapy in acute lymphoblastic leukemia patients. Cancer. Jul 1 2007;110(1):103-111. 
42. Willer A, Gerss J, Konig T, et al. Anti-Escherichia coli asparaginase antibody levels determine the activity of second-line treatment with pegylated E coli asparaginase: a retrospective analysis within the ALL-BFM trials. Blood. Nov 24 2011;118(22):5774-5782.

43. Panosyan EH, Seibel NL, Martin-Aragon S, et al. Asparaginase antibody and asparaginase activity in children with higher-risk acute lymphoblastic leukemia: Children's Cancer Group Study CCG-1961. J Pediatr Hematol Oncol. Apr 2004;26(4):217-226.

44. Larson RA, Fretzin MH, Dodge RK, Schiffer CA. Hypersensitivity reactions to Lasparaginase do not impact on the remission duration of adults with acute lymphoblastic leukemia. Leukemia. 1998;12(5):660-665.

45. Wacker P, Land VJ, Camitta BM, et al. Allergic reactions to E. coli Lasparaginase do not affect outcome in childhood B-precursor acute lymphoblastic leukemia: a Children's Oncology Group Study. J Pediatr Hematol Oncol. Sep 2007;29(9):627-632.

46. Vrooman LM, Supko JG, Neuberg DS, et al. Erwinia asparaginase after allergy to E. coli asparaginase in children with acute lymphoblastic leukemia. Pediatric blood \& cancer. Feb 2010;54(2):199-205.

47. Knoderer HM, Robarge J, Flockhart DA. Predicting asparaginase-associated pancreatitis. Pediatric blood \& cancer. Oct 15 2007;49(5):634-639.

48. Samarasinghe S, Dhir S, Slack J, et al. Incidence and outcome of pancreatitis in children and young adults with acute lymphoblastic leukaemia treated on a contemporary protocol, UKALL 2003. British journal of haematology. Sep 2013;162(5):710-713.

49. Silverman LB, Gelber RD, Dalton VK, et al. Improved outcome for children with acute lymphoblastic leukemia: results of Dana-Farber Consortium Protocol 91-01. Blood. 2001;97(5):1211-1218.

50. Raja RA, Schmiegelow K, Albertsen BK, et al. Asparaginase-associated pancreatitis in children with acute lymphoblastic leukaemia in the NOPHO ALL2008 protocol. British journal of haematology. Apr 2014;165(1):126-133.

51. Flores-Calderon J, Exiga-Gonzalez E, Moran-Villota S, Martin-Trejo J, Yamamoto-Nagano A. Acute pancreatitis in children with acute lymphoblastic leukemia treated with L-asparaginase. Journal of Pediatric

Hematology/Oncology. Oct 2009;31(10):790-793.

52. Vrooman LM, Stevenson KE, Supko JG, et al. Postinduction dexamethasone and individualized dosing of Escherichia Coli L-asparaginase each improve outcome of children and adolescents with newly diagnosed acute lymphoblastic leukemia: results from a randomized study--Dana-Farber Cancer Institute ALL Consortium Protocol 00-01. Journal of clinical oncology : official journal of the American Society of Clinical Oncology. Mar 20 2013;31(9):1202-1210.

53. Moghrabi A, Levy DE, Asselin B, et al. Results of the Dana-Farber Cancer Institute ALL Consortium Protocol 95-01 for children with acute lymphoblastic leukemia. Blood. 2007;109(3):896-904.

54. Raja RA, Schmiegelow K, Frandsen TL. Asparaginase-associated pancreatitis in children. British journal of haematology. Oct 2012;159(1):18-27. 
55. McLean R, Martin S, Lam-Po-Tang PR. Fatal case of L-asparaginase induced pancreatitis. Lancet. Dec 18 1982;2(8312):1401-1402.

56. Weetman RM, Baehner RL. Latent onset of clinical pancreatitis in children receiving L-asparaginase therapy. Cancer. Sep 1974;34(3):780-785.

57. Wu BU, Banks PA. Clinical management of patients with acute pancreatitis. Gastroenterology. Jun 2013;144(6):1272-1281.

58. Common Terminology Criteria for Adverse Events (CTCAE) Version 4.03. http://evs.nci.nih.gov/ftp1/CTCAE/CTCAE_4.03_2010-0614_QuickReference_5x7.pdf.

59. Zaheer A, Singh VK, Qureshi RO, Fishman EK. The revised Atlanta classification for acute pancreatitis: updates in imaging terminology and guidelines. Abdominal imaging. Feb 2013;38(1):125-136.

60. Kearney SL, Dahlberg SE, Levy DE, Voss SD, Sallan SE, Silverman LB. Clinical course and outcome in children with acute lymphoblastic leukemia and asparaginase-associated pancreatitis. Pediatric blood \& cancer. Aug 2009;53(2):162-167.

61. Tenner S, Baillie J, DeWitt J, Vege SS, American College of G. American College of Gastroenterology guideline: management of acute pancreatitis. The American journal of gastroenterology. Sep 2013;108(9):1400-1415; 1416.

62. Minowa K, Suzuki M, Fujimura J, et al. L-asparaginase-induced pancreatic injury is associated with an imbalance in plasma amino acid levels. Drugs in $R \& D$. Jun 1 2012;12(2):49-55.

63. Suzuki M, Shimizu T, Kudo T, Shoji H, Ohtsuka Y, Yamashiro Y. Octreotide prevents L-asparaginase-induced pancreatic injury in rats. Experimental hematology. Feb 2008;36(2):172-180.

64. Lavine RL, Brodsky I, Garofano CD, Rose LI. The effect of E. coli Lasparaginase on oral glucose tolerance and insulin release in man. Diabetologia. Aug 1978;15(2):113-116.

65. Alvarez OA, Zimmerman G. Pegaspargase-induced pancreatitis. Medical and pediatric oncology. Mar 2000;34(3):200-205.

66. Whitcomb DC, LaRusch J, Krasinskas AM, et al. Common genetic variants in the CLDN2 and PRSS1-PRSS2 loci alter risk for alcohol-related and sporadic pancreatitis. Nature genetics. Dec 2012;44(12):1349-1354.

67. Solomon S, Whitcomb DC, LaRusch J. PRSS1-Related Hereditary Pancreatitis. In: Pagon RA, Adam MP, Ardinger HH, et al., eds. GeneReviews(R). Seattle (WA)1993.

68. Teich N, Rosendahl J, Toth M, Mossner J, Sahin-Toth M. Mutations of human cationic trypsinogen (PRSS1) and chronic pancreatitis. Human mutation. Aug 2006;27(8):721-730.

69. Derikx MH, Kovacs P, Scholz M, et al. Polymorphisms at PRSS1-PRSS2 and CLDN2-MORC4 loci associate with alcoholic and non-alcoholic chronic pancreatitis in a European replication study. Gut. Sep 242014.

70. Pfutzer RH, Barmada MM, Brunskill AP, et al. SPINK1/PSTI polymorphisms act as disease modifiers in familial and idiopathic chronic pancreatitis.

Gastroenterology. Sep 2000;119(3):615-623. 
71. Aoun E, Chang CC, Greer JB, Papachristou GI, Barmada MM, Whitcomb DC. Pathways to injury in chronic pancreatitis: decoding the role of the high-risk SPINK1 N34S haplotype using meta-analysis. PloS one. 2008;3(4):e2003.

72. Felderbauer P, Hoffmann P, Einwachter H, et al. A novel mutation of the calcium sensing receptor gene is associated with chronic pancreatitis in a family with heterozygous SPINK1 mutations. BMC gastroenterology. Nov 29 2003;3:34.

73. Ooi CY, Dorfman R, Cipolli M, et al. Type of CFTR mutation determines risk of pancreatitis in patients with cystic fibrosis. Gastroenterology. Jan 2011;140(1):153-161.

74. Keiles S, Kammesheidt A. Identification of CFTR, PRSS1, and SPINK1 mutations in 381 patients with pancreatitis. Pancreas. Oct 2006;33(3):221-227.

75. Sharer N, Schwarz M, Malone G, et al. Mutations of the cystic fibrosis gene in patients with chronic pancreatitis. The New England journal of medicine. Sep 3 1998;339(10):645-652.

76. Witt $\mathrm{H}$, Luck W, Hennies HC, et al. Mutations in the gene encoding the serine protease inhibitor, Kazal type 1 are associated with chronic pancreatitis. Nature genetics. Jun 2000;25(2):213-216.

77. Szmola R, Sahin-Toth M. Chymotrypsin C (caldecrin) promotes degradation of human cationic trypsin: identity with Rinderknecht's enzyme Y. Proceedings of the National Academy of Sciences of the United States of America. Jul 3 2007;104(27):11227-11232.

78. Heap GA, Weedon MN, Bewshea CM, et al. HLA-DQA1-HLA-DRB1 variants confer susceptibility to pancreatitis induced by thiopurine immunosuppressants. Nature genetics. Oct 2014;46(10):1131-1134.

79. Felderbauer P, Klein W, Bulut K, et al. Mutations in the calcium-sensing receptor: a new genetic risk factor for chronic pancreatitis? Scandinavian journal of gastroenterology. Mar 2006;41(3):343-348.

80. Witt H, Beer S, Rosendahl J, et al. Variants in CPA1 are strongly associated with early onset chronic pancreatitis. Nature genetics. Oct 2013;45(10):1216-1220.

81. Wood NJ. Genetics: Global role for CPA1 variants in the pathogenesis of chronic pancreatitis. Nature reviews. Gastroenterology \& hepatology. Oct 2013;10(10):567.

82. Mounzer R, Whitcomb DC. Genetics of acute and chronic pancreatitis. Current opinion in gastroenterology. Sep 2013;29(5):544-551.

83. Smyth AC, Wiernik PH. Combination chemotherapy of adult acute lymphocytic leukemia. Clinical pharmacology and therapeutics. 1976;19(2):240-245.

84. Smets LA, Salomons G, van den BJ. Glucocorticoid induced apoptosis in leukemia. Advances In Experimental Medicine and Biology. 1999;457:607-614.

85. Fauci AS, Dale DC, Balow JE. Glucocorticosteroid therapy: mechanisms of action and clinical considerations. Ann.Intern.Med. 1976;84(3):304-315.

86. Inaba $\mathrm{H}$, Pui $\mathrm{CH}$. Glucocorticoid use in acute lymphoblastic leukaemia. The lancet oncology. Nov 2010;11(11):1096-1106.

87. Liu C, Kawedia JD, Cheng C, et al. Clinical utility and implications of asparaginase antibodies in acute lymphoblastic leukemia. Leukemia. Nov 2012;26(11):2303-2309. 
88. Niinimaki RA, Harila-Saari AH, Jartti AE, et al. High body mass index increases the risk for osteonecrosis in children with acute lymphoblastic leukemia. $J$ Clin.Oncol. 2007;25(12):1498-1504.

89. Mattano LA, Jr., Sather HN, Trigg ME, Nachman JB. Osteonecrosis as a complication of treating acute lymphoblastic leukemia in children: a report from the Children's Cancer Group. Journal of clinical oncology : official journal of the American Society of Clinical Oncology. 2000;18(18):3262-3272.

90. Kawedia JD, Kaste SC, Pei D, et al. Pharmacokinetic, pharmacodynamic, and pharmacogenetic determinants of osteonecrosis in children with acute lymphoblastic leukemia. Blood. Feb 24 2011;117(8):2340-2347; quiz 2556.

91. Mattano LA, Jr., Devidas M, Nachman JB, et al. Effect of alternate-week versus continuous dexamethasone scheduling on the risk of osteonecrosis in paediatric patients with acute lymphoblastic leukaemia: results from the CCG-1961 randomised cohort trial. The Lancet. Oncology. Sep 2012;13(9):906-915.

92. Patel B, Richards SM, Rowe JM, Goldstone AH, Fielding AK. High incidence of avascular necrosis in adolescents with acute lymphoblastic leukaemia: a UKALL XII analysis. Leukemia. 2008;22(2):308-312.

93. Kerachian MA, Seguin C, Harvey EJ. Glucocorticoids in osteonecrosis of the femoral head: a new understanding of the mechanisms of action. J Steroid Biochem Mol Biol. Apr 2009;114(3-5):121-128.

94. Janke LJ, Liu C, Vogel P, et al. Primary epiphyseal arteriopathy in a mouse model of steroid-induced osteonecrosis. The American journal of pathology. Jul 2013;183(1):19-25.

95. Mattano LA, Jr., Devidas M, Chen S, et al. Effect of High-Dose Methotrexate (HD-MTX) Vs Capizzi Methotrexate/Pegaspargase (C-MTX/ASNase) on Osteonecrosis (ON) Incidence in Children and Young Adults with T-Acute Lymphoblastic Leukemia (T-ALL): Results of Children's Oncology Group (COG) Study AALL0434. Blood. 2014;ASH Abstract 3649.

96. Yang L, Boyd K, Kaste SC, Kamdem Kamdem L, Rahija RJ, Relling MV. A mouse model for glucocorticoid-induced osteonecrosis: effect of a steroid holiday. J Orthop Res. Feb 2009;27(2):169-175.

97. Evans WE, Relling MV, Rodman JH, Crom WR, Boyett JM, Pui CH. Conventional compared with individualized chemotherapy for childhood acute lymphoblastic leukemia. The New England journal of medicine. 1998;338:499505.

98. Cheok MH, Lugthart S, Evans WE. Pharmacogenomics of acute leukemia. Annu Rev Pharmacol Toxicol. 2006;46:317-353.

99. Song JW, Chung KC. Observational studies: cohort and case-control studies. Plastic and reconstructive surgery. Dec 2010;126(6):2234-2242.

100. Lewallen S, Courtright P. Epidemiology in practice: case-control studies. Community eye health / International Centre for Eye Health. 1998;11(28):57-58.

101. Relling MV, Pui CH, Cheng C, Evans WE. Thiopurine methyltransferase in acute lymphoblastic leukemia. Blood. 2006;107(2):843-844.

102. Marshall E. Preventing toxicity with a gene test. Science. 2003;302(5645):588590. 
103. Tabor HK, Risch NJ, Myers RM. Candidate-gene approaches for studying complex genetic traits: practical considerations. Nat Rev Genet. May 2002;3(5):391-397.

104. Risch N, Merikangas K. The future of genetic studies of complex human diseases. Science. Sep 13 1996;273(5281):1516-1517.

105. Motsinger-Reif AA, Jorgenson E, Relling MV, et al. Genome-wide association studies in pharmacogenomics: successes and lessons. Pharmacogenetics and genomics. Aug 2013;23(8):383-394.

106. Rivas MA, Beaudoin M, Gardet A, et al. Deep resequencing of GWAS loci identifies independent rare variants associated with inflammatory bowel disease. Nature genetics. Nov 2011;43(11):1066-1073.

107. Daly AK. Genome-wide association studies in pharmacogenomics. Nature reviews. Genetics. Apr 2010;11(4):241-246.

108. Crowley JJ, Sullivan PF, McLeod HL. Pharmacogenomic genome-wide association studies: lessons learned thus far. Pharmacogenomics. Feb 2009;10(2):161-163.

109. Capizzi RL, Bertino JR, Skeel RT, et al. L-asparaginase: clinical, biochemical, pharmacological, and immunological studies. Ann Intern Med. 1971;74:893-901.

110. Clavell LA, Gelber RD, Cohen HJ, et al. Four-agent induction and intensive asparaginase therapy for treatment of childhood acute lymphoblastic leukemia. The New England journal of medicine. 1986;315:657-663.

111. Asselin BL, Ryan D, Frantz CN, et al. In vitro and in vivo killing of acute lymphoblastic leukemia cells by L-asparaginase. Cancer Res. 1989;49:4363-4368.

112. Pui CH, Evans WE. Acute lymphoblastic leukemia. The New England journal of medicine. 1998;339(9):605-615.

113. Amylon MD, Shuster J, Pullen J, et al. Intensive high-dose asparaginase consolidation improves survival for pediatric patients with $\mathrm{T}$ cell acute lymphoblastic leukemia and advanced stage lymphoblastic lymphoma: a Pediatric Oncology Group study. Leukemia. 1999;13(3):335-342.

114. Oettgen HF, Stephenson PA, Schwartz MK, et al. Toxicity of E. coli Lasparaginase in man. Cancer. 1970;25:253-278.

115. Peterson RG, Handschumacher RE, Mitchell MS. Immunological responses to Lasparaginase. The Journal of clinical investigation. 1971;50:1080-1090.

116. Evans WE, Tsiatis A, Rivera G, et al. Anaphylactoid reactions to Escherichia coli and Erwinia asparaginase in children with leukemia and lymphoma. Cancer. 1982;49:1378-1383.

117. Woo MH, Hak LJ, Storm MC, et al. Anti-asparaginase antibodies following E. coli asparaginase therapy in pediatric acute lymphoblastic leukemia. Leukemia. 1998;12(10):1527-1533.

118. Yang JJ, Cheng C, Devidas M, et al. Ancestry and pharmacogenomics of relapse in acute lymphoblastic leukemia. Nature genetics. Mar 2011;43(3):237-241.

119. Chen SH, Pei D, Yang W, et al. Genetic variations in GRIA1 on chromosome 5 q33 related to asparaginase hypersensitivity. Clinical pharmacology and therapeutics. Aug 2010;88(2):191-196. 
120. Wang B, Hak LJ, Relling MV, Pui CH, Woo MH, Storm MC. ELISA to evaluate plasma anti-asparaginase IgG concentrations in patients with acute lymphoblastic leukemia. J Immunol Methods 2000;239(1-2):75-83.

121. Swets JA. Measuring the accuracy of diagnostic systems. Science. Jun 3 1988;240(4857):1285-1293.

122. Therneau T, Grambsch PM. Modeling survival data: extending the Cox model. New York: Springer-Verlag; 2000.

123. Stein PD, Hull RD, Patel KC, et al. D-dimer for the exclusion of acute venous thrombosis and pulmonary embolism: a systematic review. Ann Intern Med. Apr 20 2004;140(8):589-602.

124. Nishimura K, Sugiyama D, Kogata Y, et al. Meta-analysis: diagnostic accuracy of anti-cyclic citrullinated peptide antibody and rheumatoid factor for rheumatoid arthritis. Ann Intern Med. Jun 5 2007;146(11):797-808.

125. Solomon DH, Kavanaugh AJ, Schur PH. Evidence-based guidelines for the use of immunologic tests: antinuclear antibody testing. Arthritis and rheumatism. Aug 2002;47(4):434-444.

126. Relling MV, Yang W, Das S, et al. Pharmacogenetic risk factors for osteonecrosis of the hip among children with leukemia. Journal of clinical oncology : official journal of the American Society of Clinical Oncology. 2004;22(19):3930-3936.

127. Sala A, Mattano LA, Jr., Barr RD. Osteonecrosis in children and adolescents with cancer - an adverse effect of systemic therapy. European journal of cancer. 2007;43(4):683-689.

128. Burger B, Beier R, Zimmermann M, Beck JD, Reiter A, Schrappe M. Osteonecrosis: a treatment related toxicity in childhood acute lymphoblastic leukemia (ALL)--experiences from trial ALL-BFM 95. Pediatr.Blood Cancer. 2005;44(3):220-225.

129. Parsons SK, Skapek SX, Neufeld EJ, et al. Asparaginase-associated lipid abnormalities in children with acute lymphoblastic leukemia. Blood. 1997;89(6):1886-1895.

130. Halton JM, Nazir DJ, McQueen MJ, Barr RD. Blood lipid profiles in children with acute lymphoblastic leukemia. Cancer. 1998;83(2):379-384.

131. Hanada T, Horigome Y, Inudoh M, Takita H. Osteonecrosis of vertebrae in a child with acute lymphocytic leukaemia during L-asparaginase therapy. European journal of pediatrics. 1989;149(3):162-163.

132. te Winkel ML, Appel IM, Pieters R, van den Heuvel-Eibrink MM. Impaired dexamethasone-related increase of anticoagulants is associated with the development of osteonecrosis in childhood acute lymphoblastic leukemia. Haematologica. Oct 2008;93(10):1570-1574.

133. Wang B, Relling MV, Storm MC, et al. Evaluation of immunologic crossreaction of antiasparaginase antibodies in acute lymphoblastic leukemia (ALL) and lymphoma patients. Leukemia. 2003;17(8):1583-1588.

134. te Winkel ML, Pieters R, Hop WC, et al. Prospective study on incidence, risk factors, and long-term outcome of osteonecrosis in pediatric acute lymphoblastic leukemia. Journal of clinical oncology : official journal of the American Society of Clinical Oncology. Nov 1 2011;29(31):4143-4150. 
135. Ribeiro RC, Fletcher BD, Kennedy W, et al. Magnetic resonance imaging detection of avascular necrosis of the bone in children receiving intensive prednisone therapy for acute lymphoblastic leukemia or non-Hodgkin lymphoma. Leukemia. 2001;15(6):891-897.

136. Arico M, Boccalatte MF, Silvestri D, et al. Osteonecrosis: An emerging complication of intensive chemotherapy for childhood acute lymphoblastic leukemia. Haematologica. 2003;88(7):747-753.

137. Kawedia JD, Janke L, Funk AJ, et al. Substrain-specific differences in survival and osteonecrosis incidence in a mouse model. Comp Med. Dec 2012;62(6):466471.

138. Fernandez CA, Cai X, Elozory A, et al. High-throughput asparaginase activity assay in serum of children with leukemia. International journal of clinical and experimental medicine. 2013;6(7):478-487.

139. Athale UH, Chan AK. Thrombosis in children with acute lymphoblastic leukemia. Part II. Pathogenesis of thrombosis in children with acute lymphoblastic leukemia: effects of the disease and therapy. Thromb.Res. 2003;111(4-5):199-212.

140. Appel IM, Hop WC, van Kessel-Bakvis C, Stigter R, Pieters R. L-Asparaginase and the effect of age on coagulation and fibrinolysis in childhood acute lymphoblastic leukemia. Thrombosis and haemostasis. Aug 2008;100(2):330-337.

141. Mitchell LG, Andrew M, Hanna K, et al. A prospective cohort study determining the prevalence of thrombotic events in children with acute lymphoblastic leukemia and a central venous line who are treated with L-asparaginase: results of the Prophylactic Antithrombin Replacement in Kids with Acute Lymphoblastic Leukemia Treated with Asparaginase (PARKAA) Study. Cancer. Jan 15 2003;97(2):508-516.

142. Diaz JA, Obi AT, Myers DD, Jr., et al. Critical review of mouse models of venous thrombosis. Arteriosclerosis, thrombosis, and vascular biology. Mar 2012;32(3):556-562.

143. Hawkins DS, Park JR, Thomson BG, et al. Asparaginase pharmacokinetics after intensive polyethylene glycol-conjugated L-asparaginase therapy for children with relapsed acute lymphoblastic leukemia. Clinical cancer research : an official journal of the American Association for Cancer Research. Aug 15 2004;10(16):5335-5341.

144. Bhojwani D, Darbandi R, Pei D, et al. Severe hypertriglyceridaemia during therapy for childhood acute lymphoblastic leukaemia. European journal of cancer. Oct 2014;50(15):2685-2694.

145. Muller HJ, Loning L, Horn A, et al. Pegylated asparaginase (Oncaspar) in children with ALL: drug monitoring in reinduction according to the ALL/NHLBFM 95 protocols. British journal of haematology. Aug 2000;110(2):379-384.

146. Muller HJ, Beier R, da Palma JC, et al. PEG-asparaginase (Oncaspar) 2500 $\mathrm{U} / \mathrm{m}(2) \mathrm{BSA}$ in reinduction and relapse treatment in the ALL/NHL-BFM protocols. Cancer Chemother Pharmacol. Feb 2002;49(2):149-154.

147. Szymanska B, Wilczynska-Kalak U, Kang MH, et al. Pharmacokinetic modeling of an induction regimen for in vivo combined testing of novel drugs against pediatric acute lymphoblastic leukemia xenografts. PloS one. 2012;7(3):e33894. 
148. Saito S, Ohzono K, Ono K. Early arteriopathy and postulated pathogenesis of osteonecrosis of the femoral head. The intracapital arterioles. Clin Orthop Relat Res. Apr 1992(277):98-110.

149. Sadr-Azodi O, Mattsson F, Bexlius TS, Lindblad M, Lagergren J, Ljung R. Association of oral glucocorticoid use with an increased risk of acute pancreatitis: a population-based nested case-control study. JAMA internal medicine. Mar 25 2013;173(6):444-449.

150. Halalsheh H, Bazzeh F, Alkayed K, Salami K, Madanat F. 6-Mercaptopurineinduced recurrent acute pancreatitis in children with acute lymphoblastic leukemia/lymphoma. Journal of pediatric hematology/oncology. Aug 2013;35(6):470-472.

151. Trivedi CD, Pitchumoni CS. Drug-induced pancreatitis: an update. J.Clin.Gastroenterol. 2005;39(8):709-716.

152. Schneider A, Larusch J, Sun X, et al. Combined bicarbonate conductanceimpairing variants in CFTR and SPINK1 variants are associated with chronic pancreatitis in patients without cystic fibrosis. Gastroenterology. Jan 2011;140(1):162-171.

153. Pui CH, Sandlund JT, Pei D, et al. Improved outcome for children with acute lymphoblastic leukemia: results of Total Therapy Study XIIIB at St Jude Children's Research Hospital. Blood. 2004;104(9):2690-2696.

154. Borowitz MJ, Devidas M, Hunger SP, et al. Clinical significance of minimal residual disease in childhood acute lymphoblastic leukemia and its relationship to other prognostic factors: A Children's Oncology Group study. Blood. 2008;111(12):5477-5485.

155. Li Y, Abecasis GR. Mach 1.0: Rapid haplotype reconstruction and missing genotype inference. Am J Hum Genet. 2006;S79:2290.

156. Kumar P, Henikoff S, Ng PC. Predicting the effects of coding non-synonymous variants on protein function using the SIFT algorithm. Nature protocols. 2009;4(7):1073-1081.

157. Adzhubei IA, Schmidt S, Peshkin L, et al. A method and server for predicting damaging missense mutations. Nat Methods. Apr 2010;7(4):248-249.

158. Chun S, Fay JC. Identification of deleterious mutations within three human genomes. Genome research. Sep 2009;19(9):1553-1561.

159. Schwarz JM, Rodelsperger C, Schuelke M, Seelow D. MutationTaster evaluates disease-causing potential of sequence alterations. Nature methods. Aug 2010;7(8):575-576.

160. Kircher M, Witten DM, Jain P, O'Roak BJ, Cooper GM, Shendure J. A general framework for estimating the relative pathogenicity of human genetic variants. Nature genetics. Mar 2014;46(3):310-315.

161. Ionita-Laza I, Lee S, Makarov V, Buxbaum JD, Lin X. Sequence kernel association tests for the combined effect of rare and common variants. American journal of human genetics. Jun 6 2013;92(6):841-853.

162. Rosendahl J, Landt O, Bernadova J, et al. CFTR, SPINK1, CTRC and PRSS1 variants in chronic pancreatitis: is the role of mutated CFTR overestimated? Gut. Apr 2013;62(4):582-592. 
163. Rosendahl J, Witt H, Szmola R, et al. Chymotrypsin C (CTRC) variants that diminish activity or secretion are associated with chronic pancreatitis. Nature genetics. Jan 2008;40(1):78-82.

164. Masson E, Chen JM, Scotet V, Le Marechal C, Ferec C. Association of rare chymotrypsinogen $\mathrm{C}$ (CTRC) gene variations in patients with idiopathic chronic pancreatitis. Human genetics. Feb 2008;123(1):83-91.

165. Hothorn T, Hornik K, Zeileis A. Unbiased recursive partitioning: A conditional inference framework. J Comput Graph Stat. Sep 2006;15(3):651-674.

166. Exome Aggregation Consortium (ExAC) data set, Cambridge, MA (URL: http://exac.broadinstitute.org [accessed Dec 2014])

167. Catasus L, Vendrell J, Aviles FX, Carreira S, Puigserver A, Billeter M. The sequence and conformation of human pancreatic procarboxypeptidase A2. cDNA cloning, sequence analysis, and three-dimensional model. The Journal of biological chemistry. Mar 24 1995;270(12):6651-6657.

168. Laethem RM, Blumenkopf TA, Cory M, et al. Expression and characterization of human pancreatic preprocarboxypeptidase A1 and preprocarboxypeptidase A2. Archives of biochemistry and biophysics. Aug 1 1996;332(1):8-18.

169. Belostotsky R, Seboun E, Idelson GH, et al. Mutations in DHDPSL are responsible for primary hyperoxaluria type III. American journal of human genetics. Sep 10 2010;87(3):392-399.

170. Monico CG, Rossetti S, Belostotsky R, et al. Primary hyperoxaluria type III gene HOGA1 (formerly DHDPSL) as a possible risk factor for idiopathic calcium oxalate urolithiasis. Clinical journal of the American Society of Nephrology : CJASN. Sep 2011;6(9):2289-2295.

171. Jiang J, Johnson LC, Knight J, et al. Metabolism of [13C5]hydroxyproline in vitro and in vivo: implications for primary hyperoxaluria. American journal of physiology. Gastrointestinal and liver physiology. Mar 15 2012;302(6):G637-643.

172. Niederau C, Niederau M, Luthen R, Strohmeyer G, Ferrell LD, Grendell JH. Pancreatic exocrine secretion in acute experimental pancreatitis. Gastroenterology. Oct 1990;99(4):1120-1127.

173. Frick TW. The role of calcium in acute pancreatitis. Surgery. Sep 2012;152(3 Suppl 1):S157-163.

174. Mitchell CJ, Playforth MJ, Kelleher J, McMahon MJ. Functional recovery of the exocrine pancreas after acute pancreatitis. Scandinavian journal of gastroenterology. Jan 1983;18(1):5-8.

175. Williams JA, Lee M. Microtubules and pancreatic amylase release by mouse pancreas in vitro. The Journal of cell biology. Dec 1976;71(3):795-806.

176. Valentijn KM, Gumkowski FD, Jamieson JD. The subapical actin cytoskeleton regulates secretion and membrane retrieval in pancreatic acinar cells. Journal of cell science. Jan 1999;112 ( Pt 1):81-96.

177. Gandhi MD, Evens AM, Fenske TS, et al. Pancreatitis in patients treated with brentuximab vedotin: a previously unrecognized serious adverse event. Blood. May 1 2014;123(18):2895-2897.

178. Hoff PM, Valero V, Holmes FA, Whealin H, Hudis C, Hortobagyi GN. Paclitaxel-induced pancreatitis: a case report. Journal of the National Cancer Institute. Jan 1 1997;89(1):91-93. 
179. Ueda T, Takeyama Y, Kaneda K, Adachi M, Ohyanagi H, Saitoh Y. Protective effect of a microtubule stabilizer taxol on caerulein-induced acute pancreatitis in rat. The Journal of clinical investigation. Jan 1992;89(1):234-243.

180. Bi Y, Page SL, Williams JA. Rho and Rac promote acinar morphological changes, actin reorganization, and amylase secretion. American journal of physiology. Gastrointestinal and liver physiology. Sep 2005;289(3):G561-570.

181. Williams JA, Chen X, Sabbatini ME. Small G proteins as key regulators of pancreatic digestive enzyme secretion. American journal of physiology. Endocrinology and metabolism. Mar 2009;296(3):E405-414.

182. Burridge K, Feramisco JR. Non-muscle alpha actinins are calcium-sensitive actinbinding proteins. Nature. Dec 10 1981;294(5841):565-567.

183. Jockusch BM, Burger MM, DaPrada M, Richards JG, Chaponnier C, Gabbiani G. alpha-Actinin attached to membranes of secretory vesicles. Nature. Dec 15 1977;270(5638):628-629.

184. Fischer J, Weide T, Barnekow A. The MICAL proteins and rab1: a possible link to the cytoskeleton? Biochemical and biophysical research communications. Mar 11 2005;328(2):415-423.

185. Giridharan SS, Caplan S. MICAL-family proteins: Complex regulators of the actin cytoskeleton. Antioxidants \& redox signaling. May 1 2014;20(13):20592073.

186. Vora A, Goulden N, Mitchell C, et al. Augmented post-remission therapy for a minimal residual disease-defined high-risk subgroup of children and young people with clinical standard-risk and intermediate-risk acute lymphoblastic leukaemia (UKALL 2003): a randomised controlled trial. The lancet oncology. Jul 2014;15(8):809-818.

187. Lopez MJ. The changing incidence of acute pancreatitis in children: a singleinstitution perspective. The Journal of pediatrics. May 2002;140(5):622-624.

188. Nydegger A, Heine RG, Ranuh R, Gegati-Levy R, Crameri J, Oliver MR. Changing incidence of acute pancreatitis: 10-year experience at the Royal Children's Hospital, Melbourne. Journal of gastroenterology and hepatology. Aug 2007;22(8):1313-1316.

189. Kloppel G, Maillet B. Pathology of acute and chronic pancreatitis. Pancreas. Nov 1993;8(6):659-670.

190. O'Donnell MD, Miller NJ. Plasma pancreatic and salivary-type amylase and immunoreactive trypsin concentrations: variations with age and reference ranges for children. Clinica chimica acta; international journal of clinical chemistry. Jul 1 1980;104(3):265-273.

191. Jordan SC, Ament ME. Pancreatitis in children and adolescents. The Journal of pediatrics. Aug 1977;91(2):211-216.

192. Haajanen J, Saarinen O, Laasonen L, Kuhlback B, Edgren J, Slatis P. Steroid treatment and aseptic necrosis of the femoral head in renal transplant recipients. Transplantation proceedings. Oct 1984;16(5):1316-1319.

193. Kerachian MA, Harvey EJ, Cournoyer D, Chow TY, Nahal A, Seguin C. A rat model of early stage osteonecrosis induced by glucocorticoids. J Orthop Surg Res. 2011;6:62. 
194. Sheridan JF, Dobbs C, Brown D, Zwilling B. Psychoneuroimmunology: stress effects on pathogenesis and immunity during infection. Clinical microbiology reviews. Apr 1994;7(2):200-212.

195. Borysenko M, Borysenko J. Stress, behavior, and immunity: animal models and mediating mechanisms. General hospital psychiatry. Apr 1982;4(1):59-67.

196. Brinks V, van der Mark M, de Kloet R, Oitzl M. Emotion and cognition in high and low stress sensitive mouse strains: a combined neuroendocrine and behavioral study in BALB/c and C57BL/6J mice. Frontiers in behavioral neuroscience. 2007;1:8.

197. Palumbo ML, Zorrilla Zubilete MA, Cremaschi GA, Genaro AM. Different effect of chronic stress on learning and memory in BALB/c and C57BL/6 inbred mice: Involvement of hippocampal NO production and PKC activity. Stress. Jul 2009;12(4):350-361.

198. Van Loo PL, Van der Meer E, Kruitwagen CL, Koolhaas JM, Van Zutphen LF, Baumans V. Long-term effects of husbandry procedures on stress-related parameters in male mice of two strains. Laboratory animals. Apr 2004;38(2):169177.

199. Olfe J, Domanska G, Schuett C, Kiank C. Different stress-related phenotypes of $\mathrm{BALB} / \mathrm{c}$ mice from in-house or vendor: alterations of the sympathetic and HPA axis responsiveness. BMC physiology. 2010;10:2.

200. Haskell CM, Canellos GP, Leventhal BG, Carbone PP, Serpick AA, Hansen HH. L-asparaginase toxicity. Cancer research. 1969;29(4):974-975.

201. Appel IM, van Kessel-Bakvis C, Stigter R, Pieters R. Influence of two different regimens of concomitant treatment with asparaginase and dexamethasone on hemostasis in childhood acute lymphoblastic leukemia. Leukemia. Nov 2007;21(11):2377-2380.

202. Merlen C, Bonnefoy A, Wagner E, et al. L-Asparaginase lowers plasma antithrombin and mannan-binding-lectin levels: Impact on thrombotic and infectious events in children with acute lymphoblastic leukemia. Pediatric blood \& cancer. Aug 2015;62(8):1381-1387.

203. Hunault-Berger M, Chevallier P, Delain M, et al. Changes in antithrombin and fibrinogen levels during induction chemotherapy with L-asparaginase in adult patients with acute lymphoblastic leukemia or lymphoblastic lymphoma. Use of supportive coagulation therapy and clinical outcome: the CAPELAL study. Haematologica. Oct 2008;93(10):1488-1494.

204. Roadmap Epigenomics C, Kundaje A, Meuleman W, et al. Integrative analysis of 111 reference human epigenomes. Nature. Feb 19 2015;518(7539):317-330.

205. Kramer A, Green J, Pollard J, Jr., Tugendreich S. Causal analysis approaches in Ingenuity Pathway Analysis. Bioinformatics. Feb 15 2014;30(4):523-530. 


\title{
APPENDIX A. SUPPLEMENTARY INFORMATION FOR CHAPTER 2
}

\author{
Anti-asparaginase Antibody ELISA
}

\section{Positive and negative controls}

An initial ELISA was achieved by using one plasma sample from an ALL patient who recently had an allergic reaction. The antibody level of this sample had an OD value of 1.0 at a 1:10000 dilution. Using this plasma sample as a positive control, and normal human plasma as a negative control, we then tested 65 samples from six ALL patients who received Elspar, Erwinase or both formulations, and of whom one received Oncaspar. Subsequently, 21 samples (from six patients) with OD values greater than 1.0 at a 1:3200 dilution were selected for establishing an anti-asparaginase antibody reference pool. After combining these 21 samples, the antibody reference was re-analyzed and then aliquoted and stored at $-80^{\circ} \mathrm{C}$. Normal human plasma (NHP) samples were obtained from young individuals (students at UTHSC) who had never received asparaginase. Informed consent was obtained from patients according to Institutional Review Board guidelines and from students according to University of Tennessee Review Board guidelines.

\section{Asparaginase antigens}

Using this anti-asparaginase antibody reference pool, the optimal concentrations of Elspar (Merck \& Co., Inc. Kenilworth, NJ), Erwinase (MRA/CAMR, Salisbury, UK) and Oncaspar (Enzon, Inc., Piscataway, NJ) commercial asparaginase preparations were standardized as distinct antigens. The ELISA was performed with these three antigens using separate well-coating concentrations ranging from 0.3125 to $40 \mu \mathrm{g} / \mathrm{ml}$ in the same plate. The antibody reference plasma was diluted 1:3200 and aliquoted to each well. NHP at $1: 3200(\mathrm{v} / \mathrm{v})$ was used as a negative control. To ensure that the antigen completely saturated the plate, the optimal concentrations of the three coating antigens were chosen as the second concentration above that defining the plateau absorbance value.

\section{Measurement of anti-asparaginase IgG antibodies by ELISA}

Microplates (Nunc-Immuno plate, purchased from Thomas Scientific Product, Swedesboro, NJ) were coated with $100 \mu \mathrm{l}$ of Elspar, Erwinase or Oncaspar commercial preparations diluted in $0.05 \mathrm{M}$ carbonate/bicarbonate buffer, $\mathrm{pH} 9.5$ (Sigma, St. Louis, $\mathrm{MO}$ ) and incubated overnight at $2-8^{\circ} \mathrm{C}$. The wells were drained without washing and blocked for at least $90 \mathrm{~min}$ at room temperature with $300 \mu \mathrm{l} /$ well of a solution of $0.1 \mathrm{M}$ phosphate-buffered saline (PBS), $\mathrm{pH} 7.2$, containing $0.1 \%$ bovine serum albumin (Sigma, St. Louis, MO) and 0.05\% Tween-20 (Sigma, St. Louis, MO). The plates were then washed three times with $0.05 \%$ Tween-20 in saline. For each asparaginase preparation, $100 \mu \mathrm{l}$ of two-fold serial dilutions of the antibody reference, NHP, or plasma samples 
with $0.05 \%$ Tween-20 in PBS were added, then incubated in appropriate wells for $1 \mathrm{~h}$ at room temperature. After incubation, the plates were again washed as described above. A polyclonal goat anti-human IgG horseradish peroxidase conjugate (specific for human $\mathrm{IgG}$, but not for IgA, IgM, Bence Jones Kappa and Lambda myeloma proteins; purchased from Sigma, St. Louis, MO) was freshly diluted to 1:10000 (v/v). A 100- $\mu$ l volume of diluted conjugate was added to each well and incubated for $1 \mathrm{~h}$ at room temperature. The wells were drained, washed with Tween-20-saline four times, and incubated with $100 \mu \mathrm{l}$ of a freshly made substrate solution containing $20 \mathrm{mg}$ of $o$-phenylenediamine dihydrochloride (OPD; purchased from Sigma, St. Louis, MO) in $50 \mathrm{ml}$ of $0.1 \mathrm{M}$ citrate buffer, $\mathrm{pH} 6.0$, and $167 \mu \mathrm{l}$ of $3 \% \mathrm{H}_{2} \mathrm{O}_{2}$ for $30 \mathrm{~min}$ at room temperature in the dark. After exactly $30 \mathrm{~min}$, a $100-\mu \mathrm{l}$ aliquot of $1.0 \mathrm{M}$ phosphoric acid solution was added to stop the reaction. Anti-asparaginase antibody concentrations were measured at $490 \mathrm{~nm}$ for the enzymatic product (subtracting the absorbance at $650 \mathrm{~nm}$ for non-specific absorbance) using a microplate reader (Model: THERMO max; purchased from Molecular Devices. LLC, Sunnyvale, CA). The cut-offs for positivity in plasma were determined as the mean plus two standard deviations of the OD values determined for plasma from healthy volunteers. Assays were performed in duplicates.

\section{Normalization of Antibody Readings}

Among the 2010 serum samples for anti-asparaginase antibodies measured by ELISA, 471 samples were assayed at UTHSC, 1345 samples were assayed at SJCRH, and 194 serum samples measured for antibody levels at both. The magnitudes of the scale of antibody OD readings obtained at UTHSC was significantly higher than those obtained at SJCRH (Table A-1 and Figure A-1). Therefore, we normalized the OD readings based on a comparison of the antibody OD readings of the 194 serum samples measured at both facilities.

A nonlinear mixed model approach was used to find the correlation between SJCRH and UTHSC readings 194 serum samples. The correlation can be described by an exponential growth curve as Equation A-1 ( $\mathrm{y}$ is the SJCRH OD reading and $\mathrm{x}$ is the relative UTHSC OD reading), or Equation A-2 (y', $x^{\prime}$ and $A_{0}$ ' are natural logarithms of $\mathrm{y}, \mathrm{x}$ and $\mathrm{A}_{0}$ ).

$$
\begin{gathered}
y=A_{0} \cdot \exp \left(A_{1} \cdot x^{k}\right) \\
y^{\prime}=A_{0}{ }^{\prime}+A_{1} \cdot \exp \left(k x^{\prime}\right)
\end{gathered}
$$

Figure A-2 shows the fitted curves for antibodies against three formulations (Elspar, Erwinase and Oncaspar). To maintain the consistency in range and threshold of the OD readings from two different facilities, the positive cut-off and the maximum OD reading were given more weight when adjusting the curve. The optimization of parameters was performed by R 2.8.0 using a nonlinear mixed model. 
Table A-1. Comparison between antibody OD readings obtained at SJCRH $(n=1539)$ and UTHSC $(n=665)$

\begin{tabular}{cccccccc}
\hline Antigen & Facility & $\begin{array}{c}\text { Positive } \\
\text { cut-off }^{\mathbf{a}}\end{array}$ & Median (range) & $\begin{array}{c}\text { Mean (SD) of } \\
\text { positive readings }\end{array}$ & $\boldsymbol{P}^{\mathbf{b}}$ & $\begin{array}{c}\text { Mean (SD) of } \\
\text { negative readings }\end{array}$ & $\begin{array}{c}\boldsymbol{P}^{\mathbf{b}} \\
\text { Elspar }\end{array}$ \\
& SJCRH & 0.036 & $0.012(0.003-1.512)$ & $0.358(0.312)$ & - & $0.011(0.007)$ & - \\
& UTHSC & 0.275 & $0.017(0,001-2.349)$ & $1.109(0.616)$ & $2 \times 10^{-16}$ & $0.032(0.058)$ & 0.11 \\
Erwinase & UTHSC (norm) & 0.037 & $0.010(0.007-1.541)$ & $0.377(0.352)$ & 0.99 & $0.011(0.006)$ & 0.15 \\
& SJCRH & 0.086 & $0.017(0.003-1.242)$ & $0.210(0.216)$ & - & $0.022(0.015)$ & - \\
& UTHSC & 0.454 & $0.016(0.001-1.387)$ & $0.756(0.362)$ & $2 \times 10^{-7}$ & $0.031(0.049)$ & $5 \times 10^{-5}$ \\
Oncaspar & UTHSC (norm) & 0.083 & $0.015(0.013-1.245)$ & $0.326(0.319)$ & 0.23 & $0.016(0.006)$ & $3 \times 10^{-8}$ \\
& SJCRH & 0.018 & $0.007(0.001-0.560)$ & $0.103(0.102)$ & - & $0.007(0.003)$ & - \\
& UTHSC & 0.125 & $0.006(0.001-1.596)$ & $0.501(0.363)$ & $2 \times 10^{-16}$ & $0.012(0.021)$ & $4 \times 10^{-6}$ \\
& UTHSC (norm) & 0.018 & $0.007(0.005-0.559)$ & $0.091(0.113)$ & 0.16 & $0.007(0.002)$ & $5 \times 10^{-5}$ \\
\hline
\end{tabular}

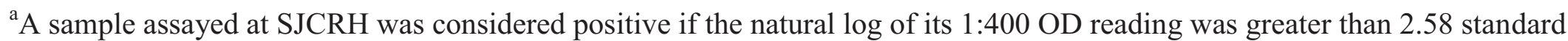
deviations above the negative control process mean. A sample assayed at UTHSC was considered positive if the natural log of its 1:3200 OD reading was greater than 2 standard deviations above the negative control process mean. Samples that were not positive were considered negative. The negative control reference pool was prepared from healthy volunteers who had never received asparaginase treatment. ${ }^{b} \mathrm{P}$-values compared with SJCRH OD readings using the Wilcoxon rank sum test. SJCRH, St. Jude Children's Research Hospital. UTHSC, University of Tennessee Health Science Center. Norm, normalized. 


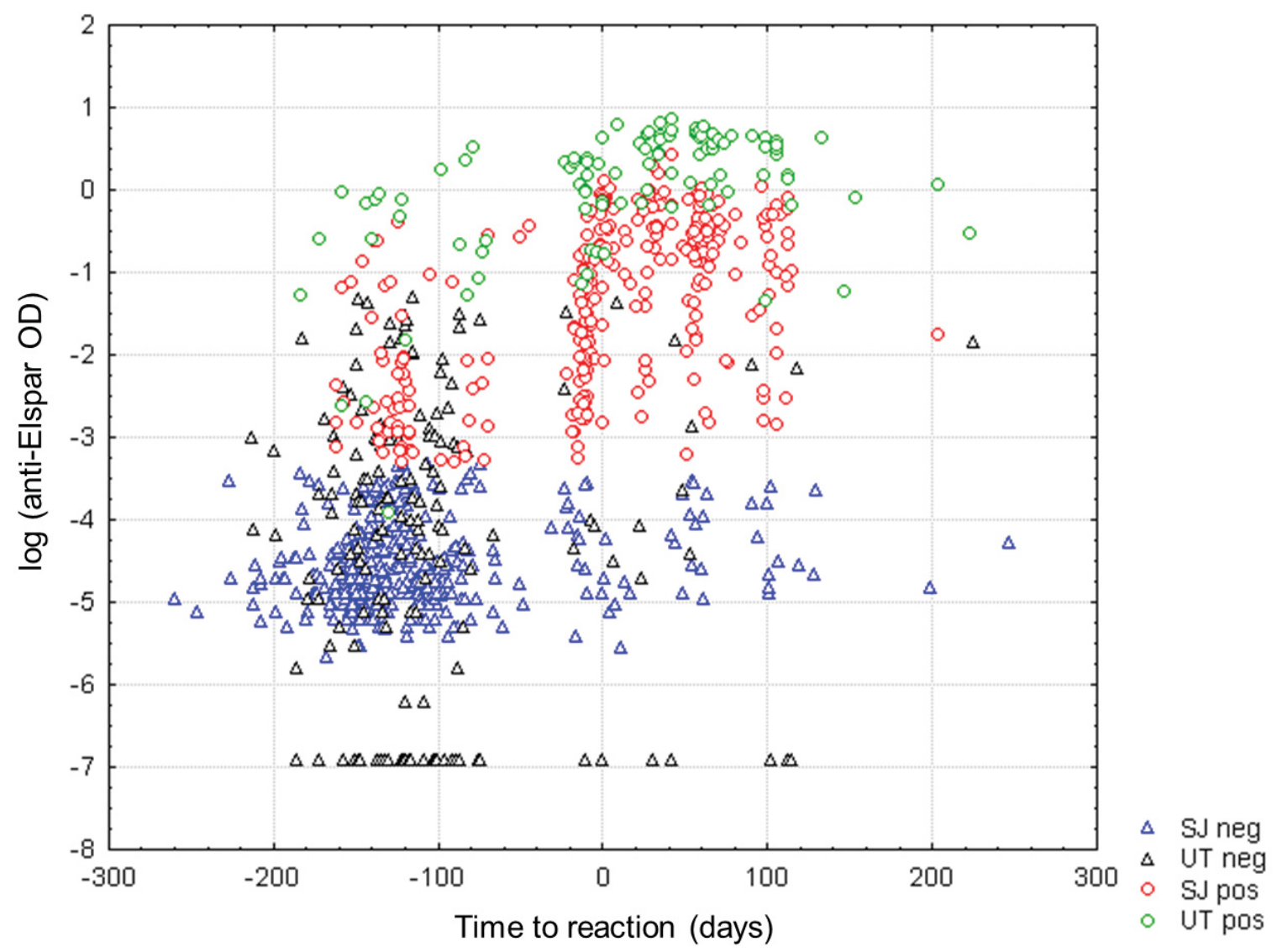

Figure A-1. Anti-Elspar OD readings obtained at SJCRH $(n=1539)$ and UTHSC $(n=665)$ relative to the time of clinical reaction

Natural logarithm of anti-Elspar OD level in patient samples obtained at SJCRH (blue triangles and red circles) and UTHSC (black triangles and green circles) relative to the number of days elapsed before (negative) or after (positive) between sample collection and clinical reaction to Elspar. SJ, St. Jude Children's Research Hospital. UT, University of Tennessee Health Science Center. Pos, positive. Neg, negative. 
Figure A-2. Fitted curves for (A) anti-Elspar, (B) anti-Erwinase and (C) antiOncaspar OD readings of the samples $(n=194)$ measured at both SJCRH and UTHSC

The positive cut-off (blue circle with cross and blue arrow), the maximum OD value (red circle and red arrow) and the fitted line are shown. 

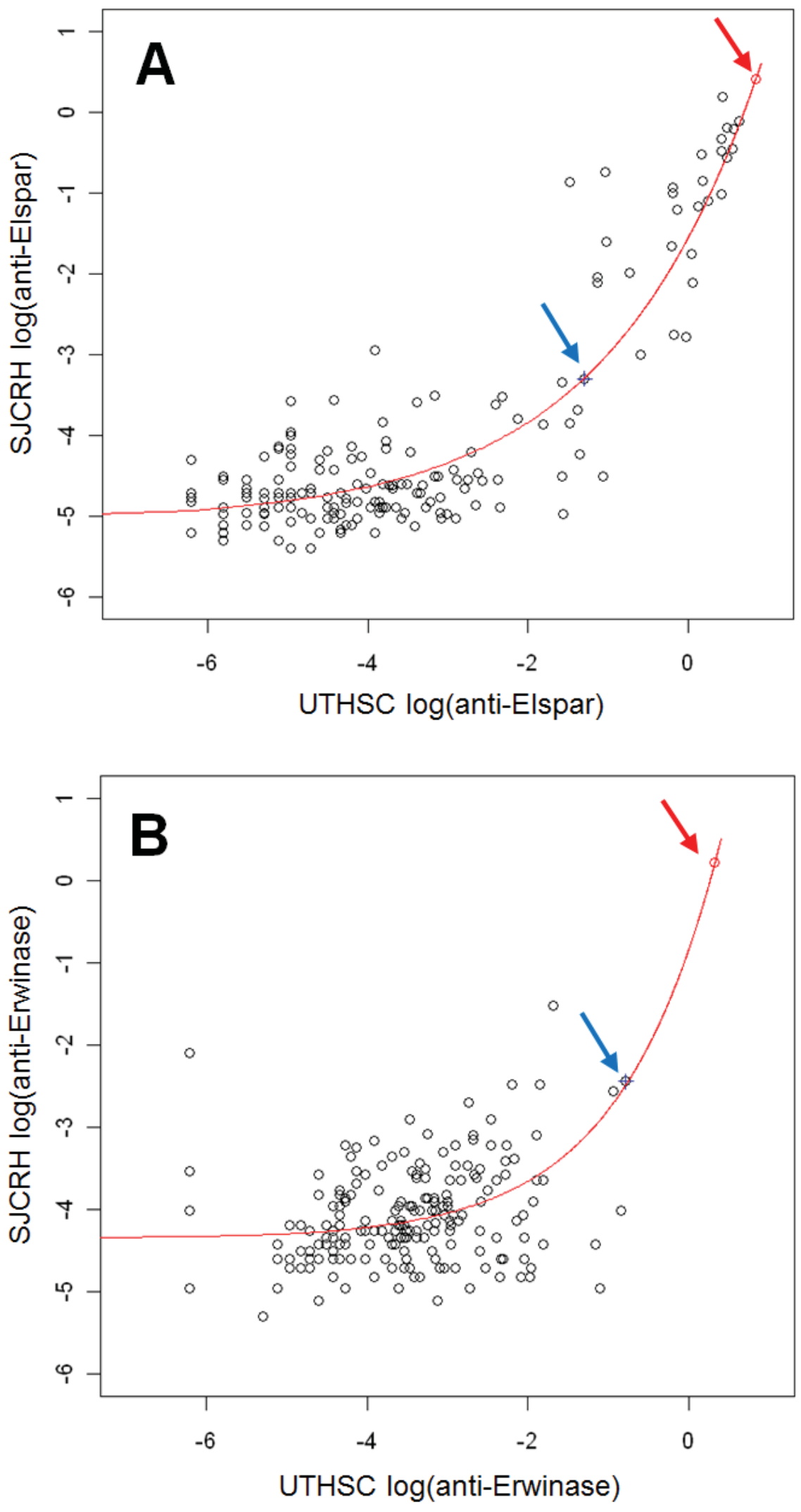


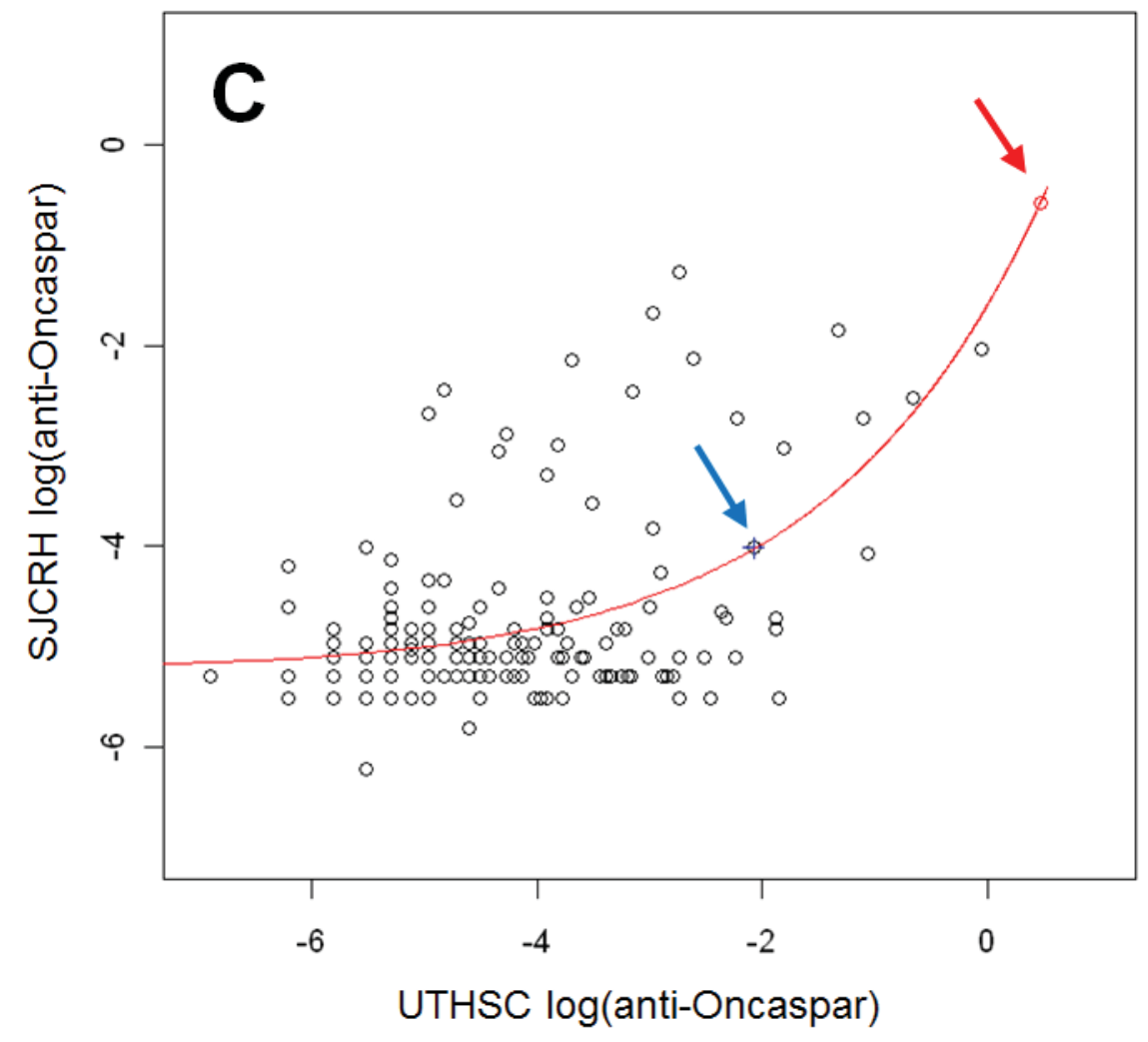

Figure A-2. (Continued) 
For anti-Elspar antibody, the (y', x') for the positive cut-off is $(-1.297,-3.326)$, and the $\left(y^{\prime}, x^{\prime}\right)$ for the maximum OD reading is $(0.854,0.414) . A_{0}{ }^{\prime}=-5.066, A_{1}=3.509$, $\mathrm{k}=0.526$ (Figure A-2A).

For anti-Erwinase antibody, the (y', $\left.x^{\prime}\right)$ for the positive cut-off is $(-0.786,-2.448)$; and the $\left(y^{\prime}, x^{\prime}\right)$ for the maximum $O D$ reading is $(0.327,0.216) . A_{0}{ }^{\prime}=-4.360, A_{1}=3.518$, $\mathrm{k}=0.806$ (Figure A-2B).

For anti-Oncaspar antibody, the (y', $\left.x^{\prime}\right)$ for the positive cut-off is $(-2.080,-4.046)$; and the $\left(\mathrm{y}^{\prime}, \mathrm{x}^{\prime}\right)$ for the maximum OD reading is $(0.468,-0.580) . \mathrm{A}_{0}{ }^{\prime}=-5.280, \mathrm{~A}_{1}=3.684$, $\mathrm{k}=0.520$ (Figure A-2C).

After normalization, the thresholds of antibody positivity and the ranges (especially for the positive OD readings) are consistent between two facilities (Table A-1 and Figure A-3). 

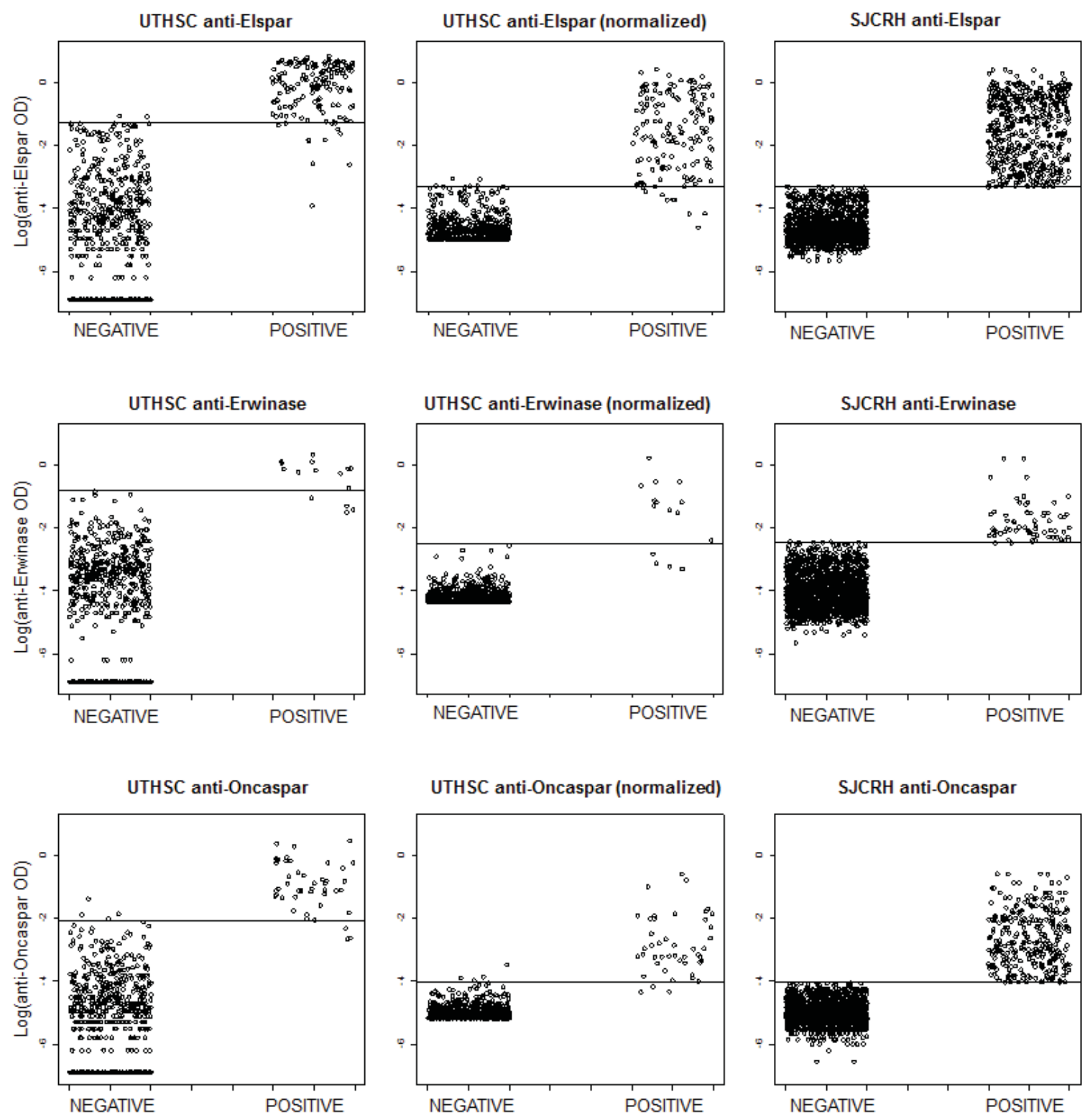

Figure A-3. UTHSC OD readings before and after normalization

The OD readings of anti-Elspar (top row), anti-Erwinase (middle row) and anti-Oncaspar (bottom row) are plotted by antibody positivity calls made by UTHSC $(n=665)$ or SJCRH $(n=1539)$. The antibody positivity thresholds are shown. Left column: OD readings obtained at UTHSC before normalization. Middle column: OD readings obtained at UTHSC after normalization. Right column: OD readings obtained at SJCRH. 


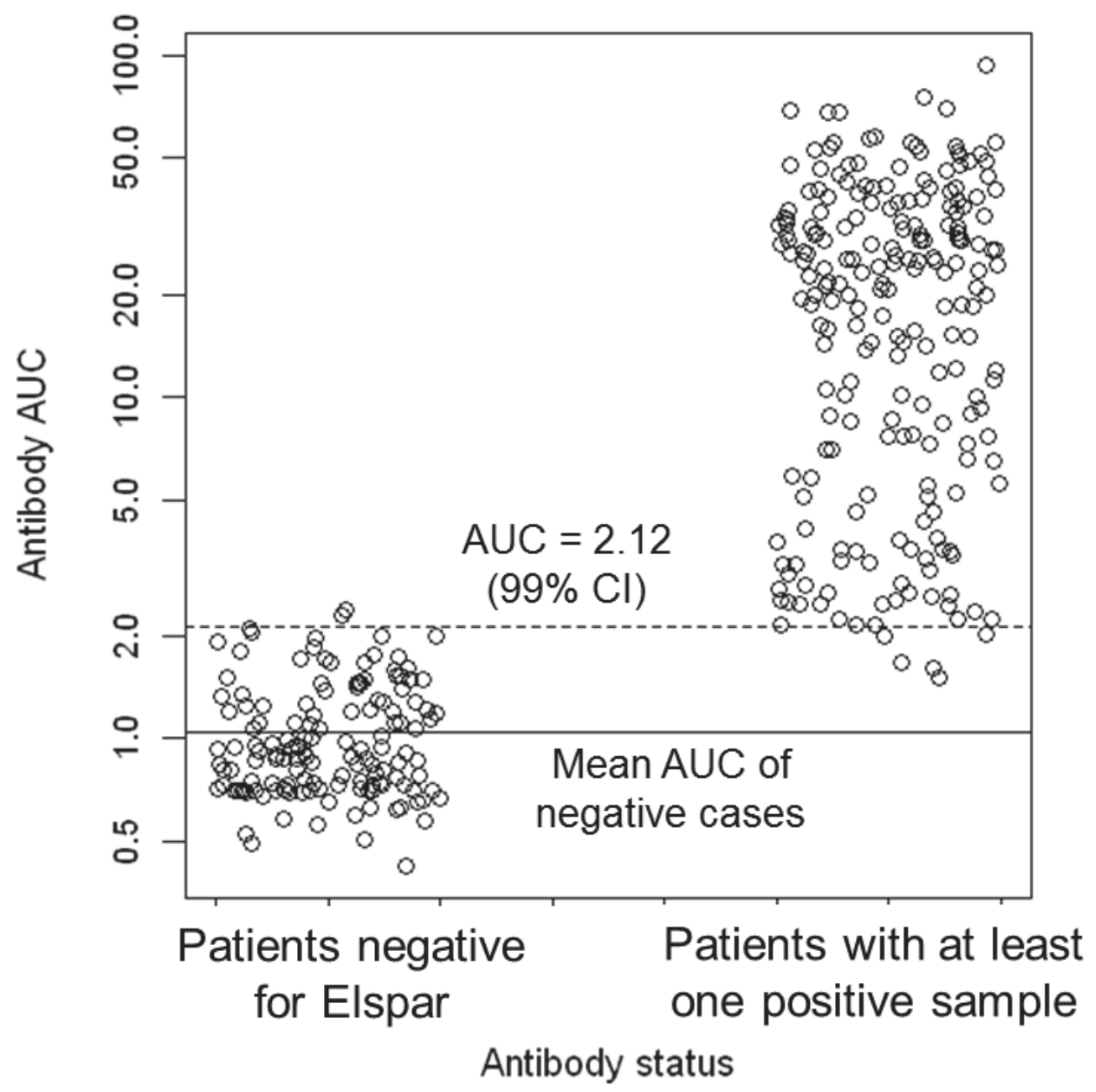

Figure A-4. Determination of AUC threshold

The AUC threshold was greater than 2.12 (OD value $\times$ days), which corresponds to $99 \%$ confidence interval (CI) or 2.58 standard deviations above the mean value of negative cases (i.e., the antibody AUCs of the patients negative for anti-Elspar antibody consistently during therapy. 
Table A-2. Sensitivity, specificity and predictive values of anti-Elspar antibody test at week 7 for predicting or confirming clinical reactions to Elspar using current positivity cutoff and optimized cutoff by recursive partitioning

\begin{tabular}{|c|c|c|c|c|c|c|c|c|c|}
\hline Risk arm & $\begin{array}{c}\mathrm{N} \text { of } \\
\text { patients }\end{array}$ & $\begin{array}{l}\text { Time of } \\
\text { Ab test }\end{array}$ & $\begin{array}{l}\text { Time of } \\
\text { Rxn }\end{array}$ & $\begin{array}{c}\text { Time between } \\
\text { test and rxn } \\
\text { (weeks) } \\
\end{array}$ & $\begin{array}{c}\text { Cutoff OD } \\
\text { value }\end{array}$ & $\begin{array}{c}\text { Sensitivity } \\
(\%)\end{array}$ & $\begin{array}{c}\text { Specificity } \\
(\%)\end{array}$ & $\begin{array}{l}\text { PPV } \\
(\%)\end{array}$ & $\begin{array}{l}\text { NPV } \\
(\%)\end{array}$ \\
\hline \multicolumn{10}{|c|}{ Current positivity cut-off } \\
\hline LR & 185 & Week 7 & Weeks 7-9 & $0-3$ & 0.037 & 87 & 68 & 73 & 85 \\
\hline SHR & 190 & Week 7 & Weeks 1-6 & $0-6$ & 0.037 & 88 & 69 & 51 & 94 \\
\hline \multicolumn{10}{|c|}{ Optimized cut-off by recursive partitioning } \\
\hline LR & 189 & Week 7 & Weeks 7-9 & $0-3$ & 0.055 & 83 & 74 & 74 & 83 \\
\hline SHR & 174 & Week 7 & Weeks 1-6 & $0-6$ & 0.050 & 89 & 71 & 50 & 95 \\
\hline
\end{tabular}

SHR, standard/high-risk; LR, low-risk; PPV, positive predictive value; NPV, negative predictive value; Ab, anti-Elspar antibody; Rxn, clinical allergic reaction; Week 7, week 7 of continuation therapy (23 weeks from diagnosis of ALL). 
W7 Ab vs. W7-9 Rxn (LR)

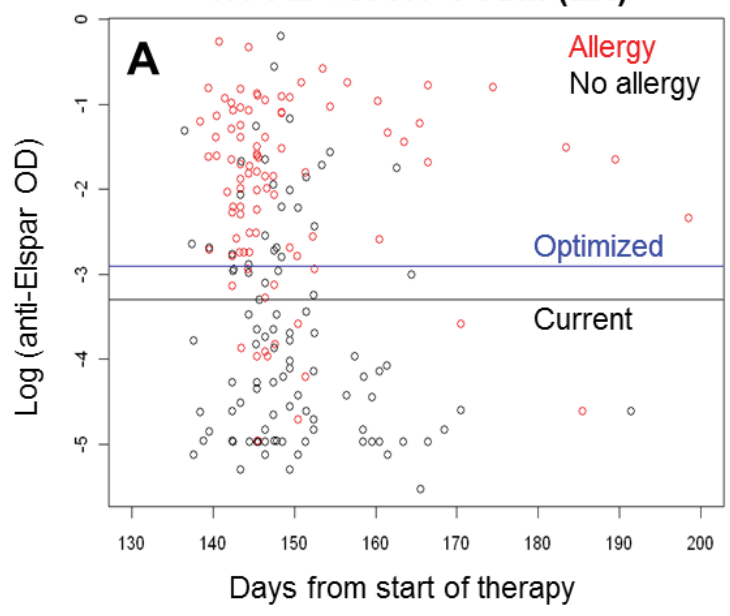

W7 Ab vs. W1-6 Rxn (SHR)

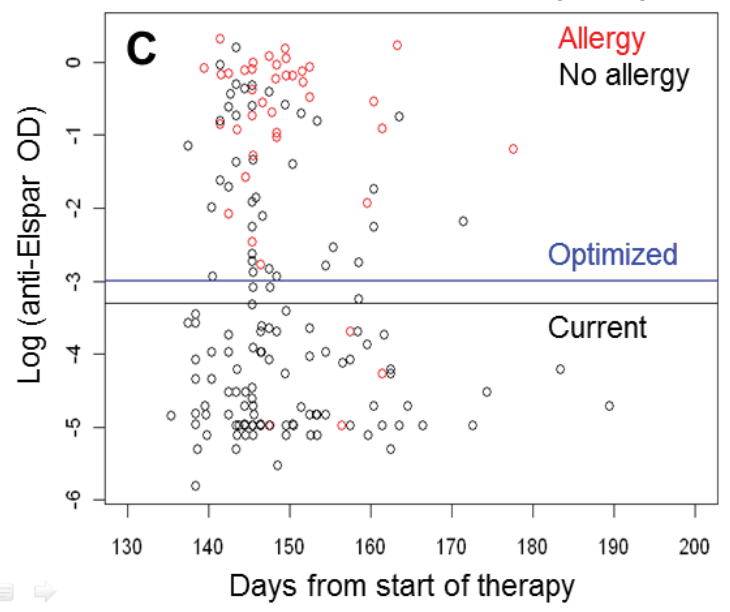

W7 Ab vs. W7-9 Rxn (LR)

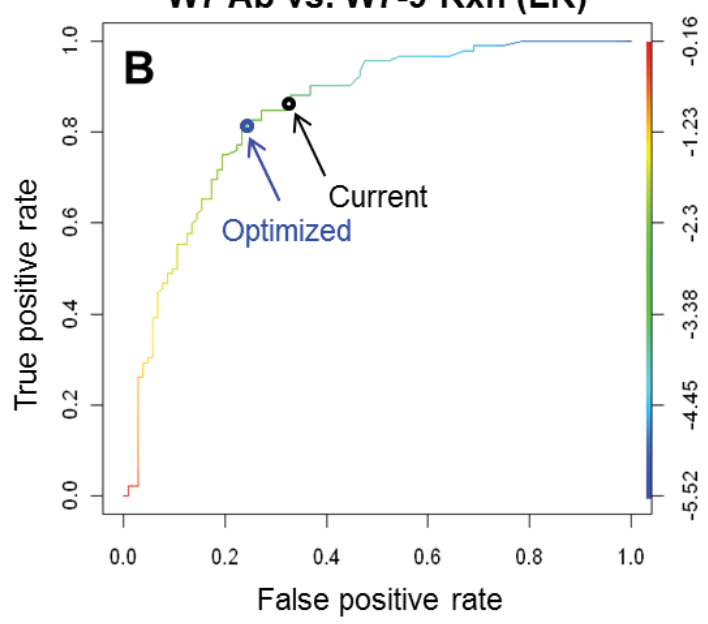

W7 Ab vs. W1-6 Rxn (SHR)

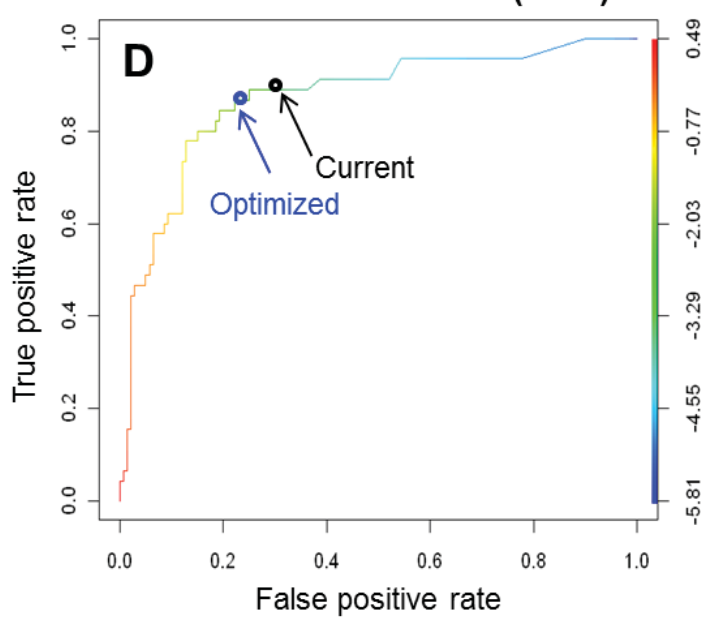

Figure A-5. Week-7 optimal threshold of OD value to predict or to confirm clinical reactions

Left: anti-Elspar OD readings of patients who had allergy (red circles) and those who did not (black circles) at week 7 were plotted against the days from start of therapy. Current antibody positivity threshold (black line) and optimized threshold (blue line) are shown. Right: the thresholds on ROC curves. The optimal thresholds to predict week 7 reactions (A and C) or to confirm week 1-6 reactions (B and D) were determined by recursive partitioning. SHR, standard/high-risk; LR, low-risk; PPV, positive predictive value; NPV, negative predictive value; $\mathrm{Ab}$, anti-Elspar antibody; Rxn, clinical allergic reaction; Week 7, week 7 of continuation therapy (about 23 weeks from diagnosis of ALL). 

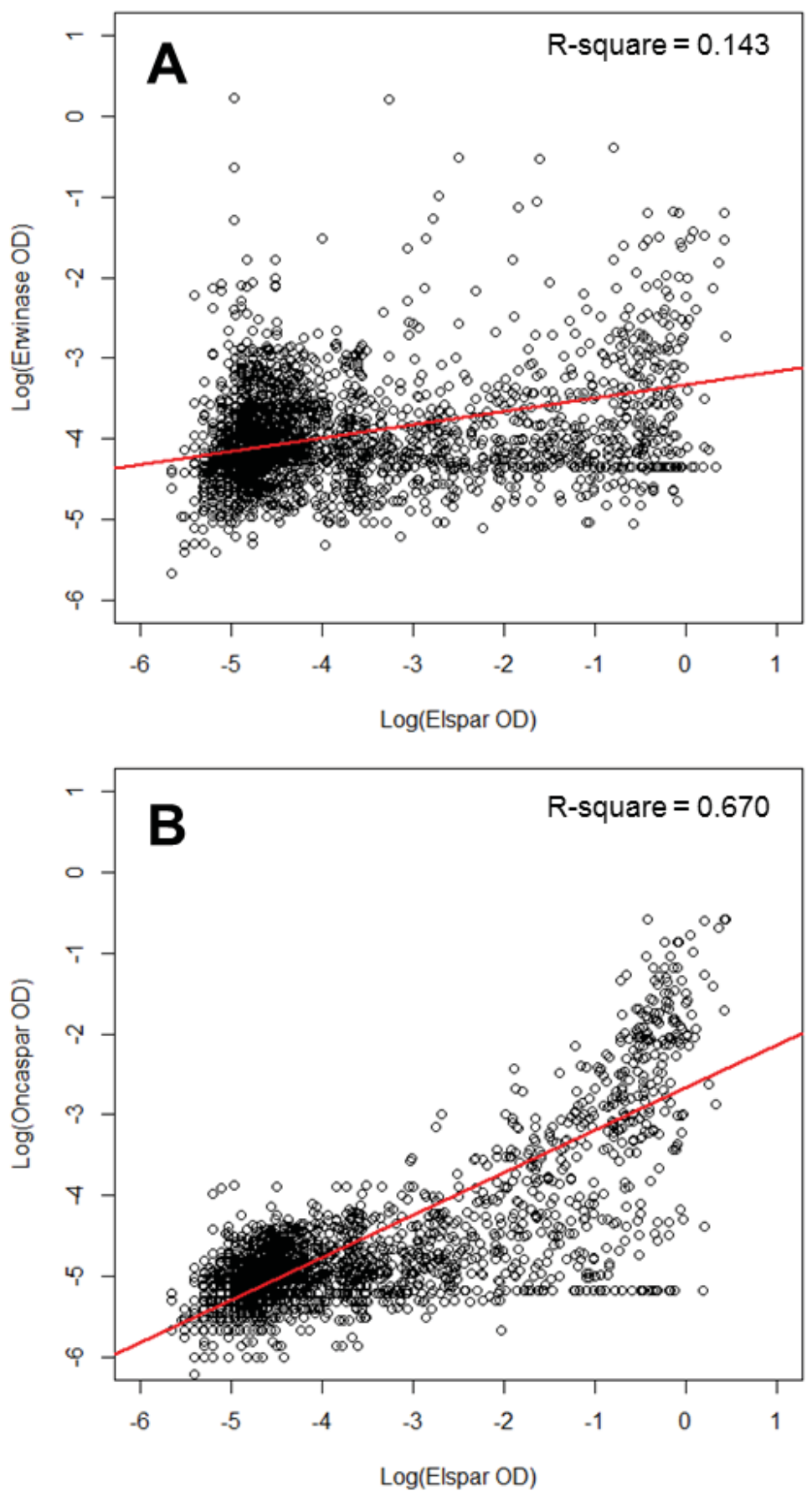

Figure A-6. Elspar has stronger cross-reactivity with Oncaspar than with Erwinase

OD readings of anti-Elspar were plotted against (A) anti-Erwinase and (B) anti-Oncaspar for each sample $(n=3597)$. Regression lines and $R$-squared values are shown. 

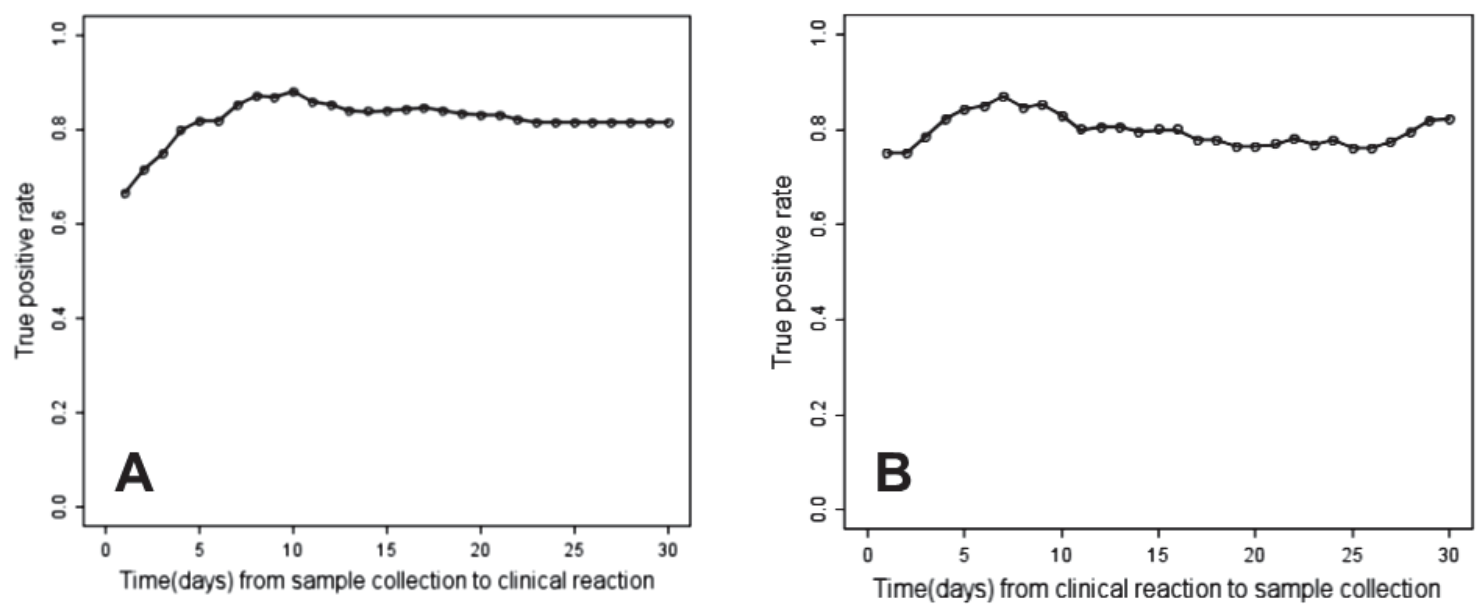

Figure A-7. Sensitivity of antibody tests within 30 days of reactions

Sensitivity of antibody tests to (A) predict future reactions $(\mathrm{n}=108)$ or $(\mathrm{B})$ confirm past reactions $(n=59)$ were plotted against the number of days within which clinical reactions occurred. Sensitivity was calculated as (number of patients who were positive for antibody within $\mathrm{n}$ days of the reaction)/(number of patients who had reactions in $\mathrm{n}$ days). 


\section{APPENDIX B. SUPPLEMENTARY INFORMATION FOR CHAPTER 3}

\section{Mouse Model of Dexamethasone-induced Osteonecrosis}

We previously showed that BALB/cJ had a higher frequency of osteonecrosis with acceptable survival rates than $\mathrm{BALB} / \mathrm{cAnN} .{ }^{137}$ Multiple risk factors of osteonecrosis were evaluated using BALB/cAnNHsd and BALB/cJ mice, including host-related factors such as age, gender, substrain and source of mice, and treatment-related factors such as dexamethasone dose and treatment duration.

\section{Methods}

\section{Chemicals}

The chemicals used for treatment have been described in Chapter 3. For dexamethasone and corcicosterone measurements, corticosterone and ammonium acetate $(98 \%, \mathrm{v} / \mathrm{v}$ in water) were purchased from Sigma (St. Louis, MO); d4_dexamethasone was from CDN Isotopes Inc. (Pointe-Claire, Quebec, Canada); acetonitrile was of HPLC grade and obtained from Burdick \& Jackson (Muskegon, MI).

\section{Animals}

All animal work conducted for this study was approved by the Institutional Animal Care and Use Committee (IACUC) at SJCRH and performed according to NIH guidelines. Male and female BALB/cJ mice (age 21 or 24 days) were shipped from Jackson Laboratories (Bar Harbor, ME), or obtained from our own breeding facilities (SJCRH, Memphis, TN). Male BALB/cAnNHsd mice (age 21 or 24 days) were obtained from Harlan Laboratories (Houston, TX). Vendor-derived mice that were 21 days old were shipped with foster mothers during transport due to the young age of the mice.

Before starting the experiment, both in-house bred and vendor-derived mice were transferred into a separate experimental room where they were maintained in sterile micro-isolator cages (Micro Vent System 75 JAG, Allentown, Allentown, NJ) and housed in ventilated racks, with up to 5 mice in a cage with corncob bedding (Andersons Bed-O'cobs, Pharmaserv, Framingham, MA). Irradiated folic-acid deficient diet (purchased from TestDiet, Richmond, IN) that contained less than $0.05 \mathrm{ppm}$ folic acid was used ${ }^{96}$. The mice had access to food and water ad libitum. Indirect health surveillance was accomplished with ICR sentinel mice (from Jackson Lab) by dirtybedding exchange weekly. The sentinels are tested quarterly and were found to be free of Sendai virus, mouse parvovirus, minute virus of mice, mouse hepatitis virus, Theiler murine encephalomyelitis virus, epizootic diarrhea of infant mice, pneumonia virus of mice, reovirus, K virus, polyoma virus, Mycoplasma pulmonis, lymphocytic 
choriomeningitis virus, mouse adenovirus, ectromelia virus, and ecto- and endoparasites. Health checks were done twice a day at a minimum and those animals that became moribund or lost $20 \%$ of their maximum body weight were immediately euthanized according to IACUC-approved procedures.

\section{Treatments}

Treatment started at postnatal day 28 (P28) or day 24 (P24). Dexamethasone was given at 4 or $8 \mathrm{mg} / \mathrm{L}$ in drinking water, equivalent to 1.33 or $2.66 \mathrm{mg} / \mathrm{kg} / \mathrm{day}$, assuming that each mouse weighed $15 \mathrm{~g}$ and consumed $5 \mathrm{~mL}$ water daily. ${ }^{137}$ Water bottles were changed every 3.5 days. Treatment generally lasted for 6 weeks, while a subset of $\mathrm{BALB} / \mathrm{cAnN}$ was treated for up to 8 weeks in an effort to increase the frequency of osteonecrosis.

Antibiotic prophylaxis to prevent dexamethasone-induced infections consisted of tetracycline $(1 \mathrm{~g} / \mathrm{L})$ constantly, and sulfamethoxazole $(600 \mathrm{mg} / \mathrm{L})$ and trimethoprim $(120$ $\mathrm{mg} / \mathrm{L})$ given 3.5 days per week. These antibiotics have no effect on osteonecrosis. ${ }^{96}$ Mice in dexamethasone groups were treated with dexamethasone with antibiotics, except in a pilot experiment where mice were given dexamethasone without antibiotics. The control groups were treated with only antibiotics.

\section{Enteric and blood cultures}

Enteric aerobic cultures were done on a single mouse per cage at the beginning and the end of experiments, and blood aerobic cultures were performed on moribund mice to determine the pathogens causing sepsis. The procedures have been described previously. $^{137}$

\section{Measurement of plasma concentration of dexamethasone and corticosterone}

An assay was developed and validated to simultaneously quantify dexamethasone and corticosterone in mouse plasma by using high-performance liquid chromatography (HPLC) with tandem mass spectrometric (MS) detection.

To $10 \mu \mathrm{L}$ of sample, $50 \mu \mathrm{L}$ of internal standard (IS; $50 \mathrm{ng} / \mathrm{mL}$ isotopically labeled $\mathrm{d}_{4}$ _dexamethasone in acetonitrile) was added and vortexed for $15 \mathrm{~min}$ at 1700rpm using a DVX-2500 multi-tube vortexer (purchased from VWR, Radnor, PA). After centrifugation at $16000 \mathrm{~g}$ for $8 \mathrm{~min}$ at $4^{\circ} \mathrm{C}$, the supernatant was transferred to an antosampler vial and 3 $\mu 1$ of the solution was injected onto the HPLC-MS system. The chromatographic separation was achieved by using a Waters (Milford, MA) UPLC with Waters ACQUITY BEHC $_{18} 1.7 \mu \mathrm{m}, 100 \times 2.1 \mathrm{~mm}$ column, and ACQUITY in-line filter as guard column. The column was maintained at $40^{\circ} \mathrm{C} \pm 5^{\circ} \mathrm{C}$ and autosampler at $15^{\circ} \pm 5^{\circ} \mathrm{C}$. The mobile phase was generated by mixing $10 \mathrm{mM}$ ammonium acetate in $\mathrm{H}_{2} \mathrm{O}$ with acetonitrile. The 
flow rate was $0.7 \mathrm{~mL} / \mathrm{min}$. The linear gradient started at time zero from $30 \%$ acetonitrile and $70 \%$ buffer, then changed to $70 \%$ acetonitrile and $30 \%$ buffer in $1.2 \mathrm{~min}$, and returned to the starting condition in a 0.01 -minute step, followed by a 1.79 min held for equilibrium. The injection interval was $3.2 \mathrm{~min}$. Detection of analytes was performed with a Waters TQD mass spectrometer operated in the positive multiple reaction monitoring (MRM) mode using Masslynx 4.1 software. The following mass ions $(\mathrm{m} / \mathrm{z})$ were used for detection: $m / z$ 393.10>373.03 for dexamethasone; $m / z$ 347.10>120.96 for corticosterone, and $\mathrm{m} / \mathrm{z} 397.10>377.09$ for IS. The following tune parameters were retained for the optimal detections: capillary voltage, $0.5 \mathrm{kV}$; cone voltage, $20 \mathrm{~V}$ for dexamethasone, $34 \mathrm{~V}$ for corticosterone, and $22 \mathrm{~V}$ for IS; source temperature, $150^{\circ} \mathrm{C}$; desolvation temperature, $450^{\circ} \mathrm{C}$; cone gas flow, $5 \mathrm{~L} / \mathrm{h}$; desolvation gas flow, $800 \mathrm{~L} / \mathrm{h}$ and collision energy, $8 \mathrm{eV}$ for Dexamethasone, $30 \mathrm{eV}$ for corticosterone, and $10 \mathrm{eV}$ for IS.

Standards were prepared in stripped mouse plasma over the linear ranges of 2 $500 \mathrm{ng} / \mathrm{ml}$ and $4-1000 \mathrm{ng} / \mathrm{ml}$ for dexamethasone and corticosterone respectively. The interday and intraday imprecision of dexamethasone at 6,80 and $400 \mathrm{ng} / \mathrm{mL}$ and corticosterone at 15,80 and $800 \mathrm{ng} / \mathrm{mL}$ was within $8.9 \%$ and the accuracy was between $98.3 \%$ and $114 \%$. The limit of quantitation (LOQ) was $5 \mathrm{nM}(2 \mathrm{ng} / \mathrm{mL})$ for dexamethasone and $11.5 \mathrm{nM}(4 \mathrm{ng} / \mathrm{mL})$ for corticosterone. At the LOQ, the interday and intraday imprecision was within $14 \%$ and accuracy was greater than $88 \%$. No interference from endogenous compounds was observed.

\section{Results}

\section{Body weight differed by substrains}

On P28, the mean weight of vendor-derived BALB/cAnNHsd males $(16.3 \pm 1.2 \mathrm{~g})$ was significantly higher than those of vendor-derived BALB/cJ males $(13.6 \pm 1.5 \mathrm{~g}, P<$ 0.0001; Figure B-1), as we reported. ${ }^{137}$ Vendor-derived BALB/cJ males were also slightly smaller $(P=0.07)$ than those raised in-house $(14.7 \pm 1.9 \mathrm{~g})$. After 6-weeks of dexamethasone treatment, there was no difference in body weight of the three colonies $(17.5 \pm 1.4 \mathrm{~g}, 17.3 \pm 1.8 \mathrm{~g}$ and $17.4 \pm 1.3 \mathrm{~g}$, respectively; $P=0.95)$. As expected, weights of dexamethasone-treated mice $(17.4 \pm 1.5 \mathrm{~g})$ were significantly lower than those of untreated controls $(26.8 \pm 2.3 \mathrm{~g} ; P<0.0001)$ at the end of treatment.

\section{Effect of substrain, source, age and treatment regimens on survival and development of osteonecrosis}

Osteonecrosis was observed as early as 4 weeks on therapy in BALB/cJ mice, and 6 weeks in BALB/cAnNHsd mice, at all tested dexamethasone doses and ages of mice at the start of therapy. The incidence of osteonecrosis tended to increase with longer treatment duration in both strains (Figure B-2). Among all the mice with osteonecrosis, 


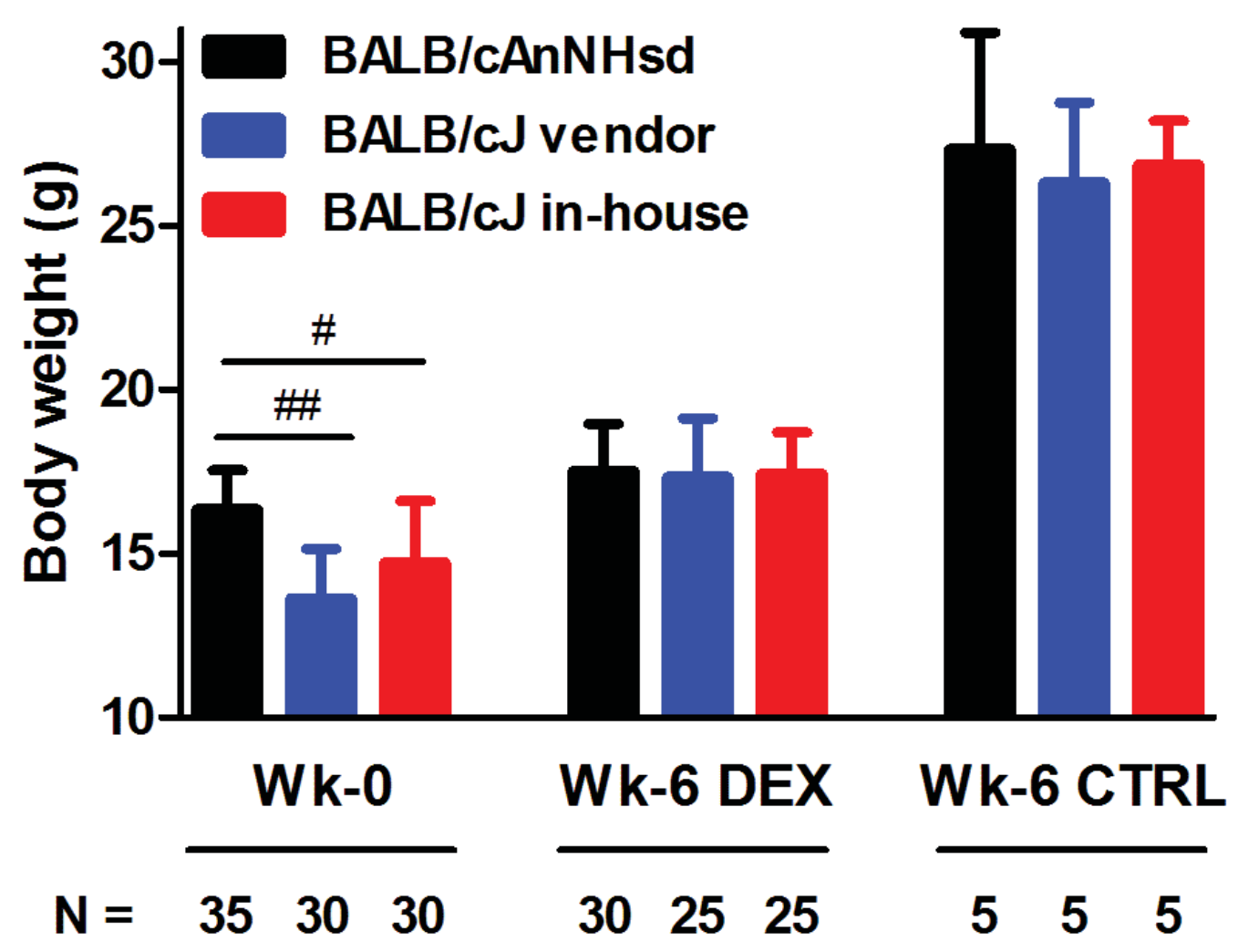

Figure B-1. Body weight of mice before and after treatment

Body weight of BALB/cAnNHsd and BALB/cJ (vendor-derived and in-house bred) male mice at the beginning of treatment $(\mathrm{P} 28, \mathrm{Wk}-0)$ and after completion of 6-week therapy (Wk-6) with dexamethasone at $4 \mathrm{mg} / \mathrm{L}$ (DEX) and their untreated controls (CTRL). $\# P<$ $0.001, \# \# P<0.0001$. 


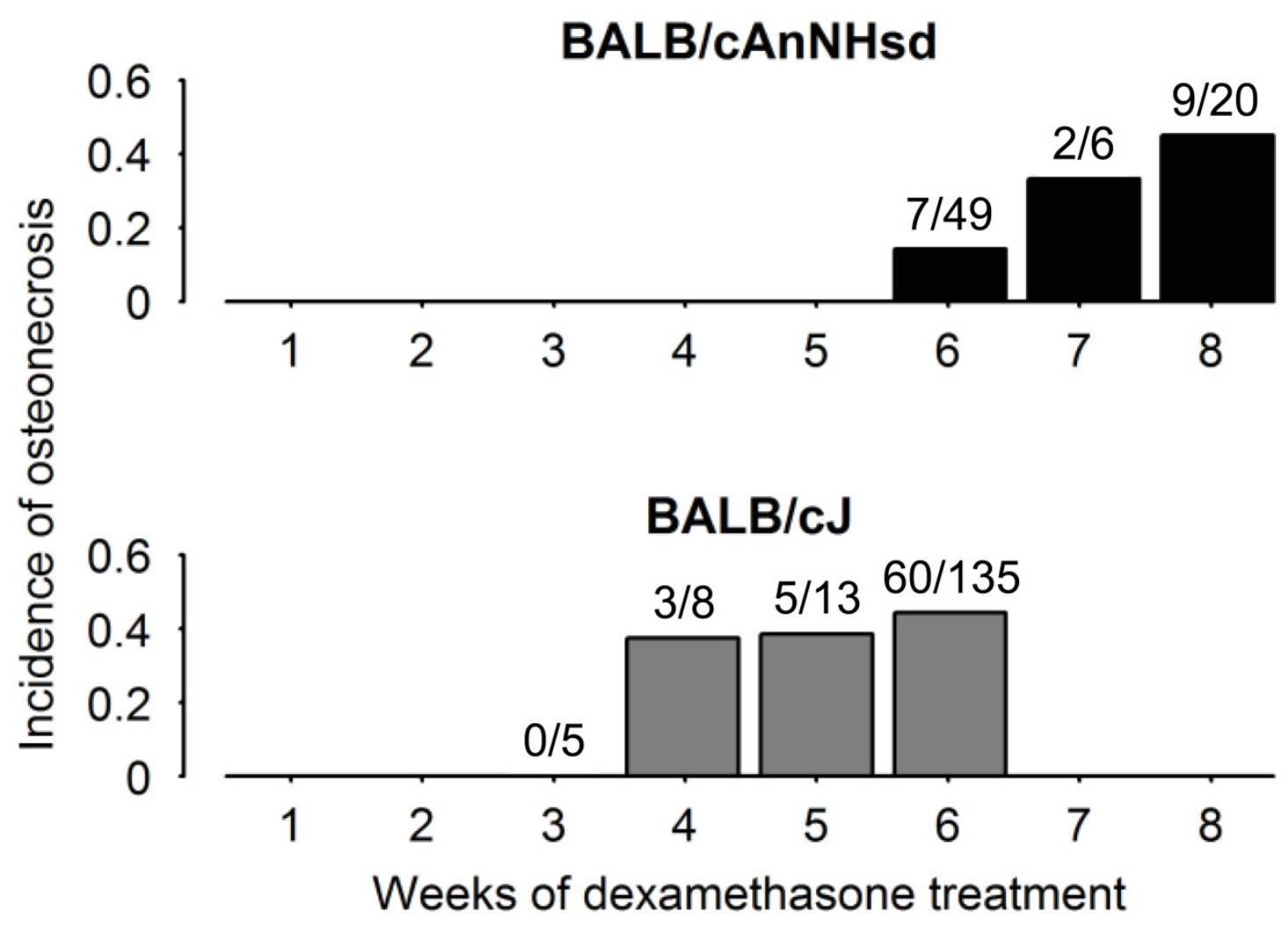

Figure B-2. Incidence of osteonecrosis increased with longer treatment duration BALB/cAnNHsd and BALB/cJ male mice from 6 experiments combined in which they were treated beginning on postnatal day 24 or 28 with either 4 or $8 \mathrm{mg} / \mathrm{L}$ dexamethasone. Evaluation of osteonecrosis at 6 weeks or later indicates that a cohort of mice had completed therapy, whereas evaluation of osteonecrosis before 6 weeks occurred because mice had to be euthanized due to morbidity (usually sepsis), and bones were evaluated at the time of sacrifice. Number of mice positive for osteonecrosis and total number mice sacrificed at each time point are shown if at least 5 mice were sacrificed at the same time point. 
the severity of osteonecrotic lesions was similar in mice of different substrain, age and treatment groups (Figure B-3).

After 6 weeks of treatment with a low dose of dexamethasone (4 mg/L), 28-daysold BALB/cAnNHsd mice did not develop any osteonecrosis. However, BALB/cJ mice of the same age had a higher incidence of osteonecrosis $(23.7 \%$ vs. $0 \%, P=0.029$; Table B-1) and significantly lower survival rate (55\% vs. $100 \%, P=0.025$; Figure B-4A) on the same dexamethasone regimen. These results are consistent with our prior report that the BALB/cAnNHsd substrain was more resistant to dexamethasone toxicity than the BALB/cJ substrain. ${ }^{137}$

We also tested whether earlier start of therapy, higher dexamethasone dose and longer treatment duration increased dexamethasone toxicity in mice, as indicated by higher incidence of osteonecrosis and lower survival rate. Earlier start of treatment (P24 vs. P28) led to a minor increase in the incidence of osteonecrosis and a significant lower survival rate in both strains (Table B-1 and Figure B-4B). Dexamethasone showed a dose-dependent toxicity in both strains. When the dose of dexamethasone was increased from $4 \mathrm{mg} / \mathrm{L}$ to $8 \mathrm{mg} / \mathrm{L}$, the incidence of osteonecrosis increased from $6.7 \%$ to $35 \%$ at 6 weeks of therapy in P24 BALB/cAnNHsd mice. Similarly, a high loading dose of $8 \mathrm{mg} / \mathrm{L}$ in the first week increased the incidence of osteonecrosis from $23.7 \%$ to $40.9 \%$ in P28 vendor-derived BALB/cJ mice at 6 weeks, and also significantly reduced the survival (28\% vs. 55\%, $P=0.016$; Figure B-4A), compared to those treated with $4 \mathrm{mg} / \mathrm{L}$ without a loading dose. Finally, longer treatment duration seems to slightly increase the incidence of osteonecrosis in P24 BALB/cAnNHsd mice (45\% at 8 weeks vs. 35\% at 6 weeks).

Blood cultures on moribund mice were positive for intestinal bacteria (e.g. Enterococcus faecalis), indicating that translocation of intestinal bacteria was responsible for sepsis-related morbidity in these dexamethasone-induced immunosuppressed mice. Antibiotics were necessary to decrease the incidence of sepsis, as a small group of $\mathrm{BALB} / \mathrm{cJ}$ mice receiving dexamethasone without prophylactic antibiotics all had to be sacrificed due to sepsis within two weeks (Figure B-4A).

An interesting observation was that BALB/cJ mice derived from in-house breeding had significantly better survival (75\% vs. 55\%, $P=0.016$; Figure B-4A) and higher incidence of osteonecrosis ( $49.5 \%$ vs. $23.7 \%, P=0.025$; Table B-1) than did those shipped from the vendor. To determine whether the difference in sepsis-related morbidity was associated with different gut flora in the two colonies, we identified bacterial isolates by enteric aerobic culture at the start and at the end of therapy, and tested their antimicrobial sensitivity (Table B-2). At the start of therapy, isolates from both colonies (e.g. E. faecalis, Staphylococcus xylosus and Lactococcus lactis) were sensitive to the antibiotics (tetracycline and trimethoprim) used in our experiments. At the end of therapy, vendor-derived mice developed resistant E. faecalis at the end of therapy, while all isolates cultured from the in-house bred mice before and after therapy were sensitive to trimethoprim. Resistant $E$. faecalis was also present in the blood of moribund vendor-derived BALB/cJ mice. 

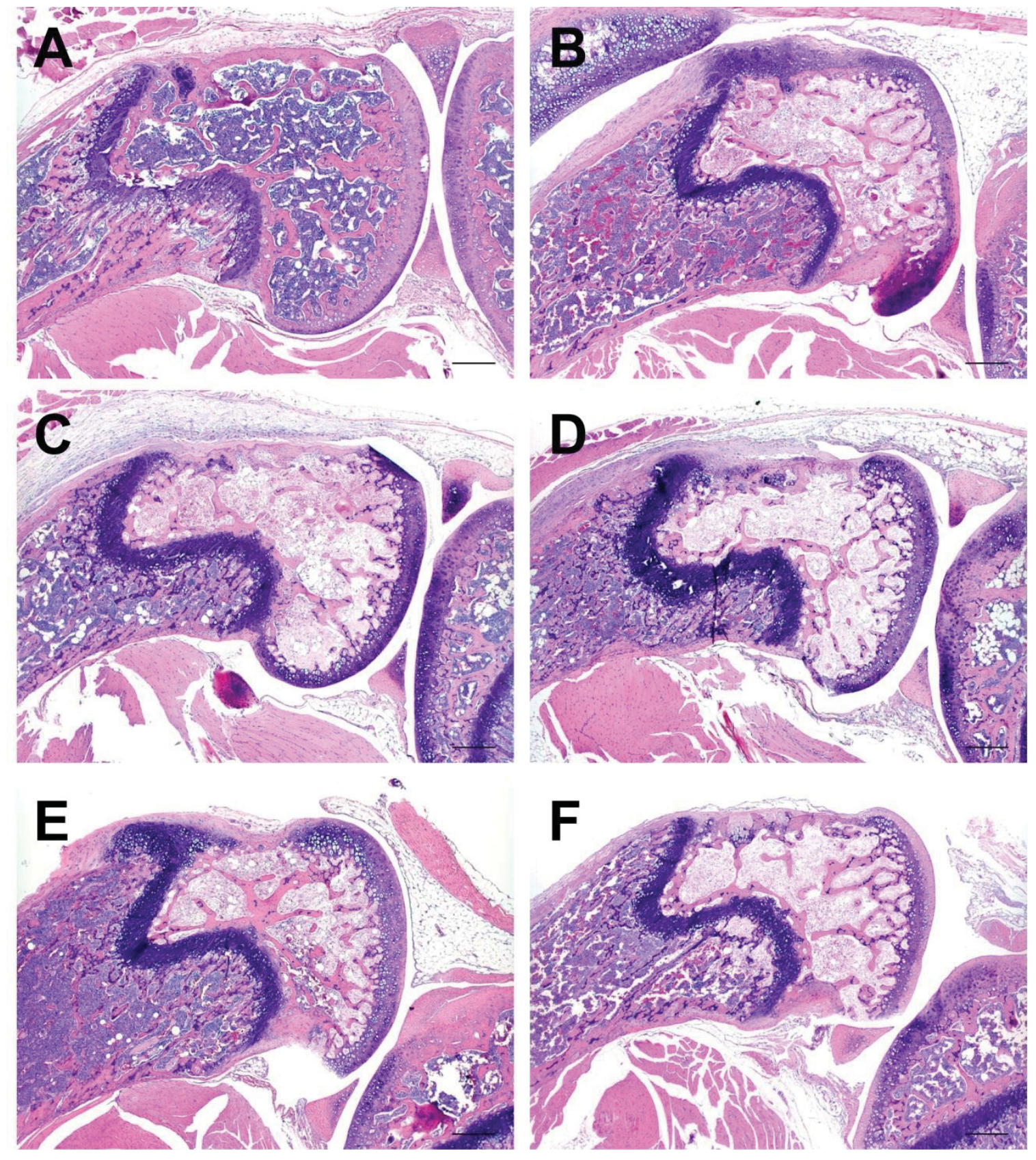

Figure B-3. Similar histologic appearance of osteonecrosis in mice of different substrain, age and treatment groups

Normal stifle joint from (A) an untreated vendor-derived BALB/cJ mouse, and osteonecrotic joints from (B) P24 BALB/cAnNHsd on $8 \mathrm{mg} / \mathrm{L}$; (C) P28 vendor-derived $\mathrm{BALB} / \mathrm{cJ}$ on $4 \mathrm{mg} / \mathrm{L}$; (D) P28 vendor-derived BALB/cJ on $8 \mathrm{mg} / \mathrm{L}$ for the first week and $4 \mathrm{mg} / \mathrm{L}$ thereafter; (E) P24 vendor-derived BALB/cJ on $8 \mathrm{mg} / \mathrm{L}$ for the first week and 4 $\mathrm{mg} / \mathrm{L}$ thereafter; (F) P28 in-house bred BALB/cJ on $4 \mathrm{mg} / \mathrm{L}$ dexamethasone. All mice were treated for 6 weeks. Magnification: $4 \times$. 
Table B-1. Incidence of $\mathrm{ON}$ in male BALB/c mice from different experiments

\begin{tabular}{cccccc}
\hline Substrain & Source & Age & DEX & $\begin{array}{c}\text { Treatment } \\
\text { duration } \\
\text { (weeks) }\end{array}$ & $\begin{array}{c}\text { Frequency of } \mathbf{O N}^{\mathbf{a}} \\
\text { (n positive/ } \\
\text { n analyzed) }\end{array}$ \\
\hline BALB/cAnNHsd & Vendor & P28 & $4 \mathrm{mg} / \mathrm{L}$ & 6 & $0 \%(0 / 20)$ \\
BALB/cAnNHsd & Vendor & P24 & $4 \mathrm{mg} / \mathrm{L}$ & 6 & $6.7 \%(1 / 15)$ \\
BALB/cAnNHsd & Vendor & P24 & $8 \mathrm{mg} / \mathrm{L}$ & 6 & $35.0 \%(7 / 20)$ \\
BALB/cAnNHsd & Vendor & P24 & $8 \mathrm{mg} / \mathrm{L}$ & 8 & $45.0 \%(9 / 20)$ \\
BALB/cJ & Vendor & P28 & $4 \mathrm{mg} / \mathrm{L}$ & 6 & $23.7 \%(6 / 26)$ \\
BALB/cJ & Vendor & P28 & $8 / 4 \mathrm{mg} / \mathrm{L}$ & 6 & $40.9 \%(9 / 22)$ \\
BALB/cJ & Vendor & P24 & $8 / 4 \mathrm{mg} / \mathrm{L}$ & 6 & $45.4 \%(5 / 11)$ \\
BALB/cJ & In-house & P28 & $4 \mathrm{mg} / \mathrm{L}$ & 6 & $49.5 \%(48 / 97)$ \\
\hline
\end{tabular}

BALB/cAnNHsd mice were shipped from vendor (Harlan Lab). BALB/cJ mice were shipped from vendor (Jackson Lab) or bred in-house (SJCRH). All mice received dexamethasone alone (DEX; 4 or $8 \mathrm{mg} / \mathrm{L}$ in drinking water). ${ }^{\mathrm{a}}$ Osteonecrosis (ON) was evaluated in mice treated for at least 4 weeks. ${ }^{b}$ Dexamethasone was given at $8 \mathrm{mg} / \mathrm{L}$ for the first week and $4 \mathrm{mg} / \mathrm{L}$ thereafter. P28, postnatal day 28. P24, postnatal day 24. 
Figure B-4. Survival differed by substrains, sources, regimens and age of mice (A) Kaplan-Meier curves of vendor-derived BALB/cJ, in-house bred BALB/cJ, and vendor-derived BALB/cAnNHsd male mice treated with different dexamethasone regimens beginning on postnatal day 28 . BALB/cJ mice were given either $4 \mathrm{mg} / \mathrm{L}$ throughout the 6-week treatment period (low-dose), or $8 \mathrm{mg} / \mathrm{L}$ for the first week and 4 $\mathrm{mg} / \mathrm{L}$ thereafter (high-dose). All BALB/cAnNHsd mice were on the low-dose regimen. Note that all mice except a small group $(n=4)$ received prophylactic antimicrobials to prevent infection. (B) Kaplan-Meier curves of vendor-derived BALB/cJ and

$\mathrm{BALB} / \mathrm{cAnNHsd}$ males with dexamethasone treatment beginning on postnatal day 28 (solid lines) or 24 (dashed lines). BALB/cJ mice received high-dose regimen, and $\mathrm{BALB} / \mathrm{cAnNH}$ sd mice received low-dose regimen. 

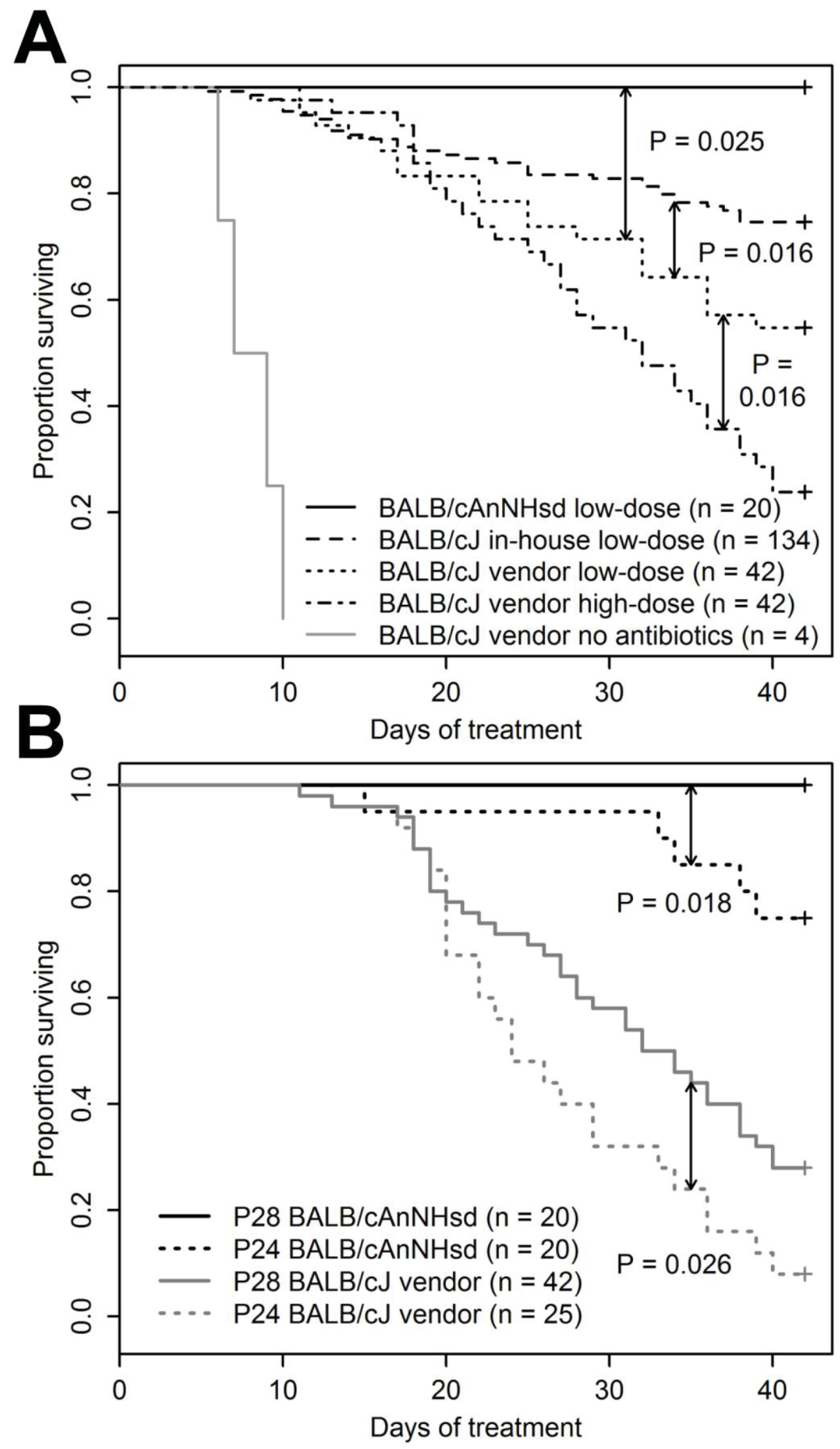
Table B-2. Enteric culture isolates from BALB/cJ mice from vendor and in-house breeding colony

\begin{tabular}{|c|c|c|c|c|c|c|}
\hline \multirow[b]{2}{*}{ Time } & \multicolumn{3}{|c|}{ Vendor-derived } & \multicolumn{3}{|c|}{ In-house bred } \\
\hline & Isolate & $\begin{array}{c}\text { TCN } \\
\text { sensitivity }\end{array}$ & $\begin{array}{c}\text { TMP } \\
\text { sensitivity }\end{array}$ & Isolate & $\begin{array}{c}\text { TCN } \\
\text { sensitivity }\end{array}$ & $\begin{array}{c}\text { TMP } \\
\text { sensitivity }\end{array}$ \\
\hline \multirow[t]{3}{*}{ Week 0} & Enterococcus faecalis & $\mathrm{R}$ & $\mathrm{S}$ & Enterococcus faecalis & $\mathrm{S}$ & $\mathrm{S}$ \\
\hline & Staphylococcus xylosus & $\mathrm{S}$ & $\mathrm{S}$ & Lactococcus lactis & $\mathrm{S}$ & $\mathrm{R}$ \\
\hline & Staphylococcus epidermidis & $\mathrm{S}$ & $\mathrm{R}$ & Escherichia coli & $\mathrm{S}$ & S \\
\hline \multirow[t]{2}{*}{ Week 6} & Enterococcus faecalis & $\mathrm{R}$ & $\mathrm{R}$ & Enterococcus faecalis & $\mathrm{R}$ & $\mathrm{S}$ \\
\hline & Enterococcus gallinarum & $\mathrm{R}$ & $\mathrm{S}$ & Enterococcus gallinarum & $\mathrm{R}$ & $\mathrm{S}$ \\
\hline
\end{tabular}

The isolates and their sensitivity to tetracycline (TCN) and trimethoprim (TMP) before the start of therapy (Week 0$)$ and after completion of therapy (Week 6) are shown. R, resistant; S, sensitive. 


\section{Dexamethasone pharmacokinetics and pharmacodynamics}

To investigate the pharmacokinetics and pharmacodynamics of dexamethasone in mice, we measured plasma concentration of dexamethasone and corticosterone in $\mathrm{BALB} / \mathrm{cAnNHsd}$ and BALB/cJ males on the same dexamethasone regimen $(4 \mathrm{mg} / \mathrm{L})$. Plasma dexamethasone levels were significantly higher in in-house bred BALB/cJ mice than in vendor-derived $\mathrm{BALB} / \mathrm{cJ}(P=0.04)$ and $\mathrm{BALB} / \mathrm{cAnNHsd}$ mice $(P=0.0002$; Figure B-5A). This is consistent with the high incidence of osteonecrosis in in-house bred BALB/cJ mice (Table B-1). Furthermore, dexamethasone levels were higher in mice with osteonecrosis than those without osteonecrosis $(P=0.013$; Figure B-5B). These plasma levels are comparable to pediatric ALL patients receiving $8-12 \mathrm{mg} / \mathrm{m}^{2}$ dexamethasone. ${ }^{30}$ As a marker of dexamethasone pharmarcodynamic effect, plasma corticosterone was suppressed in all dexamethasone-treated mice compared with untreated controls (Figure B-6).

\section{Gender-dependent difference in susceptibility to osteonecrosis}

After 6 weeks of treatment with dexamethasone $(4 \mathrm{mg} / \mathrm{L})$, the survival of in-house bred BALB/cJ mice did not differ significantly between males and females, but tended to be lower in males (75\% vs. $80 \%, P=0.3$; Figure B-7A). However, only 5 of $78(6.4 \%)$ females developed osteonecrosis, whereas 48 of 97 (49.5\%) males developed osteonecrosis $(P<0.0001$; Figure B-7B). Plasma concentrations of dexamethasone were comparable between male mice $(61.4 \pm 37.5 \mathrm{nM})$ and female mice $(69.3 \pm 39.7 \mathrm{nM}, P=$ 0.1 ; Figure B-7C), and corticosterone was completely suppressed $(<11.5 \mathrm{nM})$ in both male and female mice. Thus, the gender-dependent difference in susceptibility of osteonecrosis cannot be attributed to different pharmacokinetics or pharmacodynamics of dexamethasone.

\section{Discussion}

This is the first study to evaluate the effect of multiple risk factors on development of glucocorticoid-induced osteonecrosis in a mouse model. We report that earlier age of treatment (P24 vs. P28), male sex, higher dose of dexamethasone, longer treatment duration and higher plasma dexamethasone levels were associated with osteonecrosis in $\mathrm{BALB} / \mathrm{c}$ mice, an osteonecrosis -susceptible strain we reported previously. ${ }^{96}$ With phenotypes such as osteonecrosis that require prolonged treatment with immunosuppressive medications, it is a challenge to derive regimens that induce the target phenotype (osteonecrosis) without causing undue "off-target" toxicities (such as sepsis). Understanding the myriad factors that contribute to desired and undesired drug effects in this mouse model is thus critical.

Age has been known to be a critical risk factor for osteonecrosis in ALL patients. Clinical studies reported that adolescents (10-20 years old) were at higher risk than 


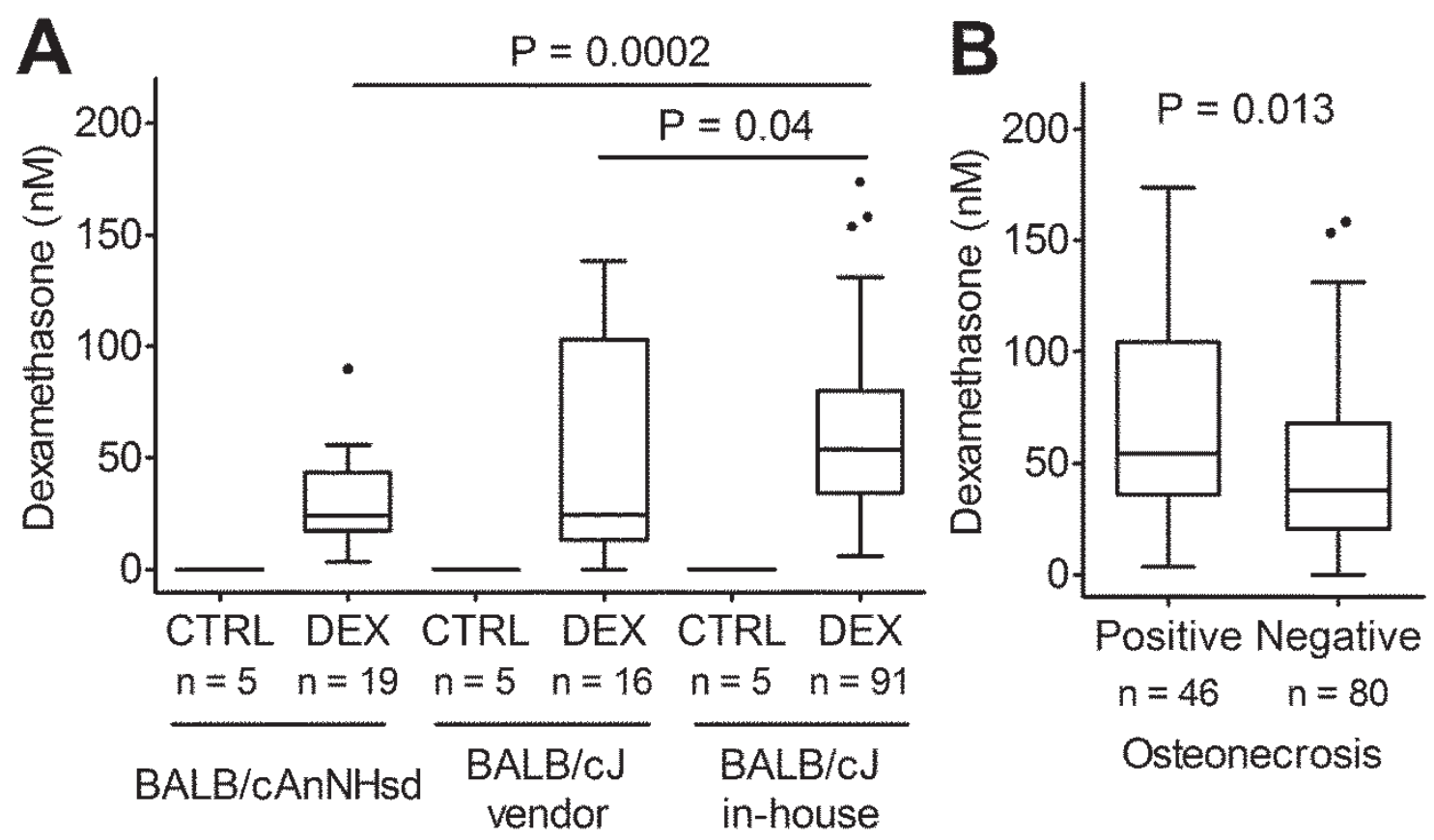

Figure B-5. Plasma concentrations of dexamethasone

Dexamethasone concentrations measured at the time of euthanasia in BALB/cJ males (vendor-derived vs. in-house bred) treated with dexamethasone (DEX) administered at 4 $\mathrm{mg} / \mathrm{L}$. 


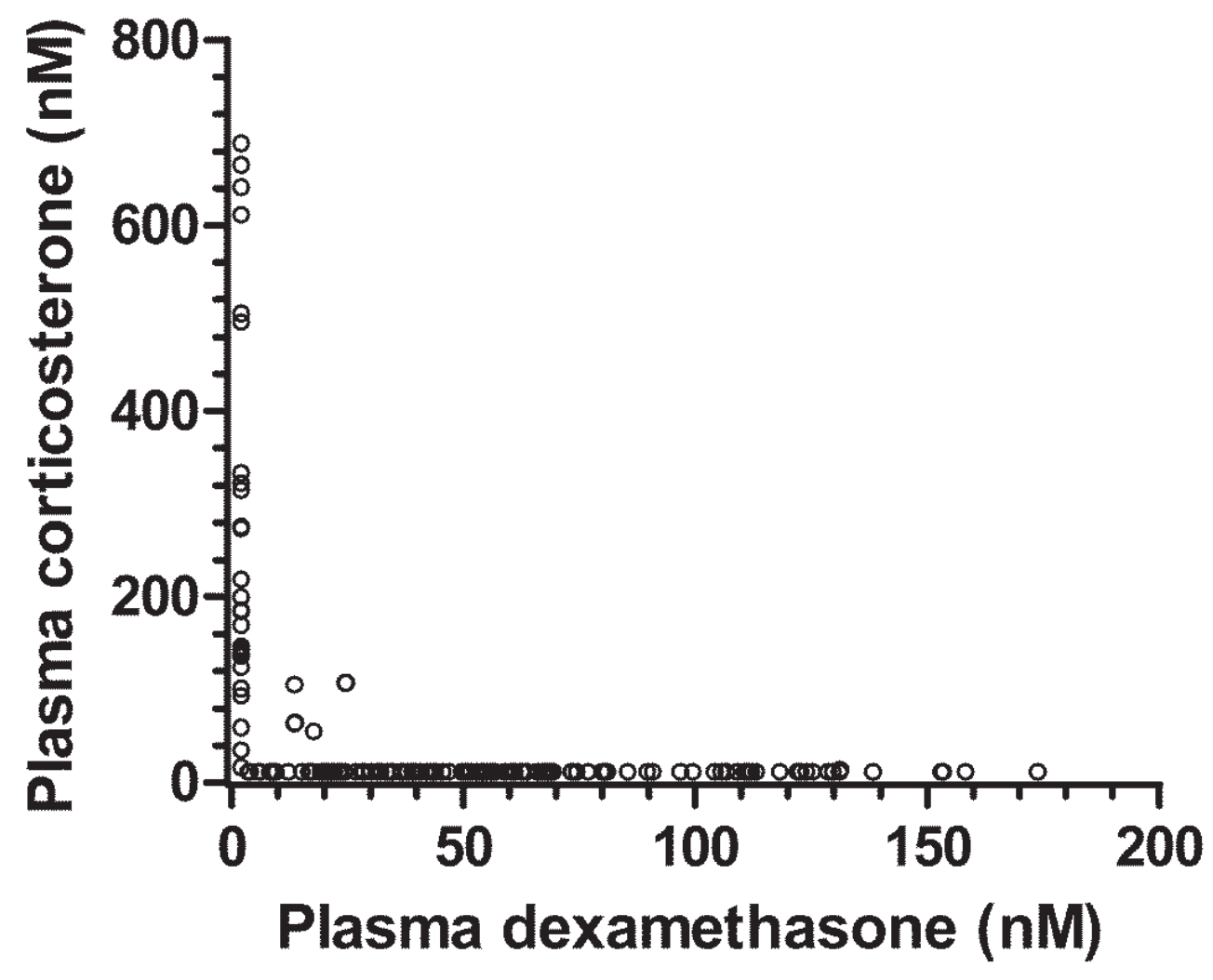

Figure B-6. Inverse association between plasma corticosterone and dexamethasone levels

Individual data points are shown for all male BALB/cAnNHsd and BALB/cJ mice treated with 0 or $4 \mathrm{mg} / \mathrm{L}$ dexamethasone at the time of euthanasia $(\mathrm{n}=167)$. 

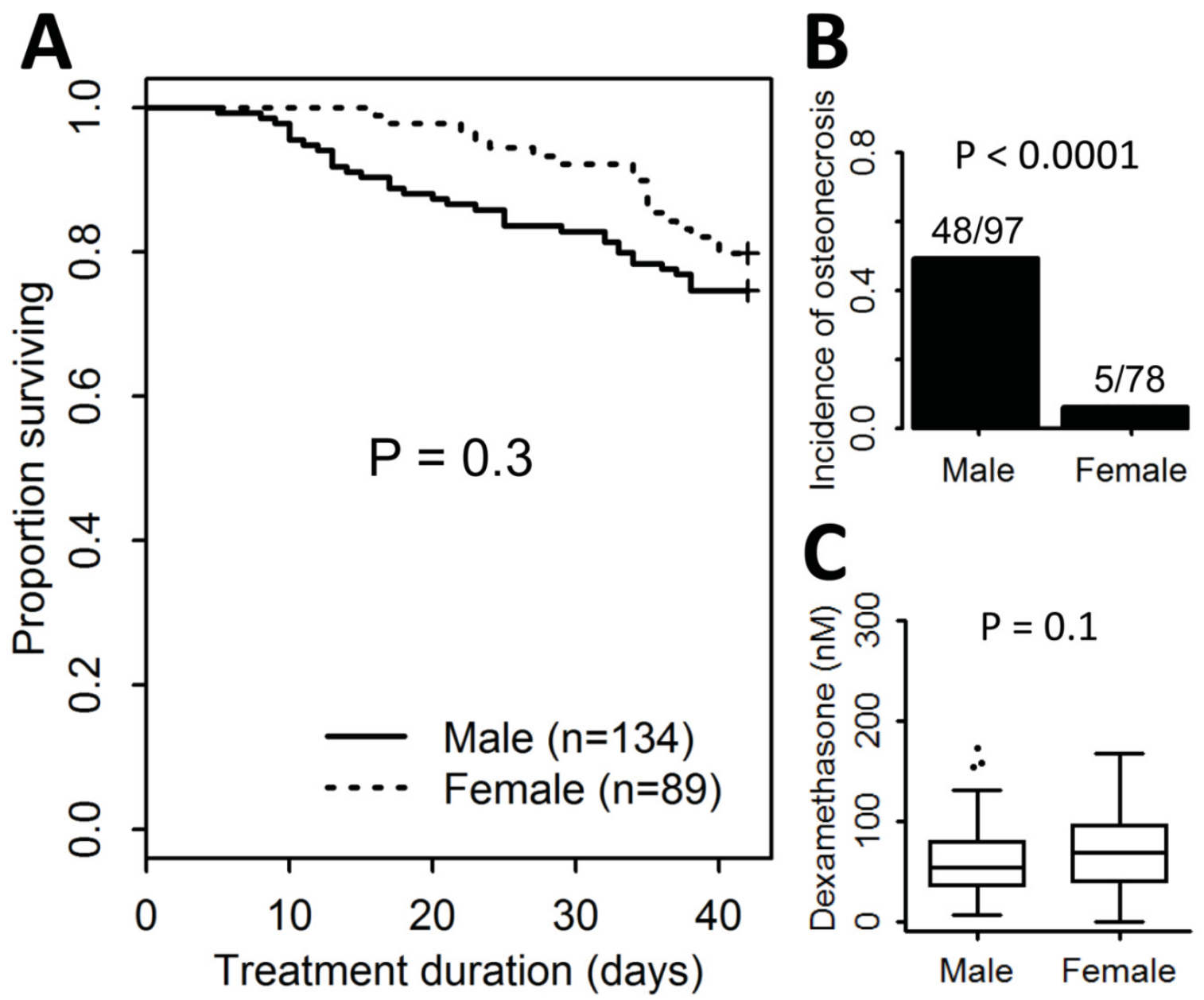

Figure B-7. Gender-dependent differences in susceptibility to osteonecrosis (A) Kaplan-Meier curve of in-house bred BALB/cJ males and females treated with dexamethasone at $4 \mathrm{mg} / \mathrm{L}$. (B) Frequency of osteonecrosis in male and female mice treated with dexamethasone at $4 \mathrm{mg} / \mathrm{L}$ for 4-6 weeks. (C) Plasma dexamethasone concentration in in-house bred BALB/cJ males and females treated with dexamethasone at $4 \mathrm{mg} / \mathrm{L}$. 
children less than 10 years old ${ }^{88-92,126,128,134-136}$ and adults over 20 years old. ${ }^{92}$ It is hypothesized that hormonal and physiologic changes during puberty, and the skeletal maturation, may contribute to their susceptibility to osteonecrosis ${ }^{192}$. The impact of age on the development of osteonecrosis was recapitulated in our mouse model. We have determined that the onset of puberty in BALB/c males occurs at postnatal $28 \pm 1$ days (data not published). In a pilot study, osteonecrosis was mostly observed in mice that started treatment between 4 and 8 weeks of age, with a decreasing incidence in older mice, regardless of dexamethasone dose and treatment duration (data not published). In the present study we showed that younger mice (24 days old) were slightly more susceptible to osteonecrosis than older mice (28 days old, Table B-1). We hypothesize that starting treatment right before onset of puberty probably maintained plasma dexamethasone at high levels for the whole pubertal period, resulting in an increased incidence of osteonecrosis in mice. However, earlier treatment significantly increased dexamethasone toxicity, and resulted in high mortality of BALB/cJ mice due to sepsis (Figure B-4B).

Strain- and substrain-specific differences in susceptibility to glucocorticoidinduced osteonecrosis are observed in mice and rats and can be attributed to different genetic backgrounds. ${ }^{96,137,193}$ An earlier study showed substrain-specific differences in survival and osteonecrosis incidence in a small group of vender-derived BALB/cJ and $\mathrm{BALB} / \mathrm{cAnNHsd}$ mice. ${ }^{137}$ In this experiment we replicated these findings using a larger group of mice treated on multiple regimens. We found that BALB/cAnNHsd mice had higher body weight and better survival, and were also less susceptible to osteonecrosis than BALB/cJ, suggesting that BALB/cJ substrain, rather than BALB/cAnNHsd, may serve as an appropriate mouse model for studying glucocorticoid-induced osteonecrosis.

Here, for the first time, we showed that $\mathrm{BALB} / \mathrm{cJ}$ mice shipped from a vendor (at 21-24 days of age) had different responses to dexamethasone compared with those from in-house breeding colonies. When treated on the same regimen, we observed higher mortality and lower incidence of osteonecrosis in BALB/cJ mice shipped from a vendor than those bred in our own facility. Our first hypothesis for the high mortality of vendorderived mice is the stress caused by transportation and unfamiliar environment. The relationship between stress and disease susceptibility has been demonstrated in many animal models, ${ }^{194,195}$ and BALB/c was relatively stress-vulnerable among several strains. ${ }^{196-198}$ Olfe et al ${ }^{199}$ also showed that transportation during the juvenile life caused a long-term stress-induced lymphocytopenia in vendor-derived BALB/c mice compared with those derived from in-house breeding. This is consistent with our observation that vendor-derived BALB/c mice had more sepsis-related morbidity. The other hypothesis is that mice shipped from a vendor vs. bred in-house would have different gastrointestinal flora, and differing microbial sensitivity to prophylactic antibiotics may also have contributed to the difference in survival rates. Lethal gut-derived sepsis was likely caused by resistant $E$. faecalis, which was isolated from enteric cultures from healthy vendorderived BALB/cJ mice, and blood cultures from moribund vendor-derived BALB/cJ mice after dexamethasone. Our experiments suggested that in-house bred BALB/cJ males were most susceptible to osteonecrosis with acceptable toxicity in response to 
dexamethasone treatment, and therefore may serve as an appropriate model for glucocorticoid-induced osteonecrosis.

As we expected, osteonecrosis was associated with higher dexamethasone exposure, including higher dose, longer treatment duration, and higher plasma concentration of dexamethasone (Table B-1 and Figure B-5B). This is consistent with clinical observations that osteonecrosis was associated with intense glucocorticoid treatment and lower dexamethasone clearance, ${ }^{88-92}$ As we reported previously, ${ }^{137}$ the resistance to dexamethasone-induced osteonecrosis in vendor-derived BALB/cAnNHsd mice compared to vendor-derived $\mathrm{BALB} / \mathrm{cJ}$ mice was not due to dexamethasone pharmacokinetics (Figure B-5A). However, in-house bred BALB/cJ mice had higher $(P$ $=0.04$ ) plasma dexamethasone levels than did either group of vendor-derived mice. The lower plasma dexamethasone concentration in vendor-derived mice was consistent with the lower incidence of osteonecrosis compared with those raised in-house $(23.7 \% \mathrm{vs}$. 49.5\%; Table B-1).

There are conflicting clinical data regarding the gender-dependent difference in risk of glucocorticoid-induced osteonecrosis in patients. Some studies reported that girls had higher risk than boys, ${ }^{88,89,91,134,136}$ but the difference was absent in several other studies. ${ }^{90,92,128}$ The incidence of osteonecrosis in mice, unlike the patients, was significantly higher among males than females after 6 weeks of treatment with $4 \mathrm{mg} / \mathrm{L}$ dexamethasone $(P<0.0001$; Figure B-7B). There were no significant difference between males and females in the survival rates and plasma levels of dexamethasone and corticosterone, suggesting some intrinsic sensitivity to osteonecrosis (e.g. sex hormones, bone or vasculature properties) may predispose male mice to osteonecrosis.

\section{Conclusion}

In summary, we report that development of dexamethasone-induced osteonecrosis in mice was affected by multiple factors, including age, gender, dexamethasone dose, treatment duration, substrain and sources of mice. Four-week-old in-house bred BALB/cJ male mice displayed high susceptibility to osteonecrosis with an acceptable survival rate; therefore they may serve as a reliable and reproducible mouse model for studying glucocorticoid-induced osteonecrosis in the future. 
Effect of Asparaginase Treatment on Dexamethasone Pharmacokinetics

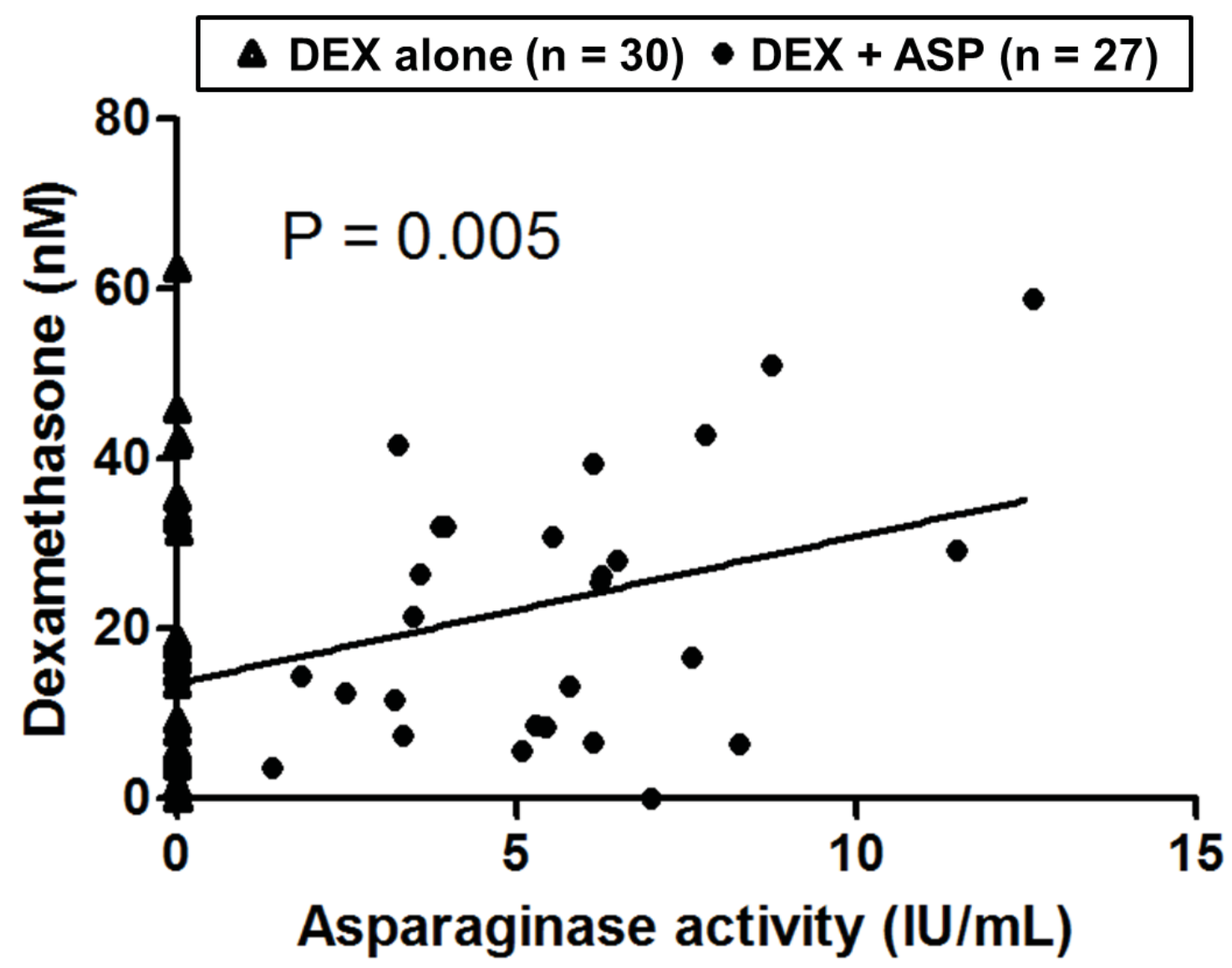

Figure B-8. Plasma dexamethasone concentration was positively associated with asparaginase activity in osteonecrosis experiment Mice received dexamethasone (DEX; $4 \mathrm{mg} / \mathrm{L}$ in drinking water) for 6 weeks (triangles) or dexamethasone and PEG-asparaginase (ASP; $1200 \mathrm{IU} / \mathrm{kg}$ i.p.) at 3.5 day intervals (12 doses in total) for 6 weeks (points). Samples were collected at the end of week 6 . Each dot represents a mouse. Linear regression line is shown. 
Effect of asparaginase and dexamethasone on plasma protein and lipid levels

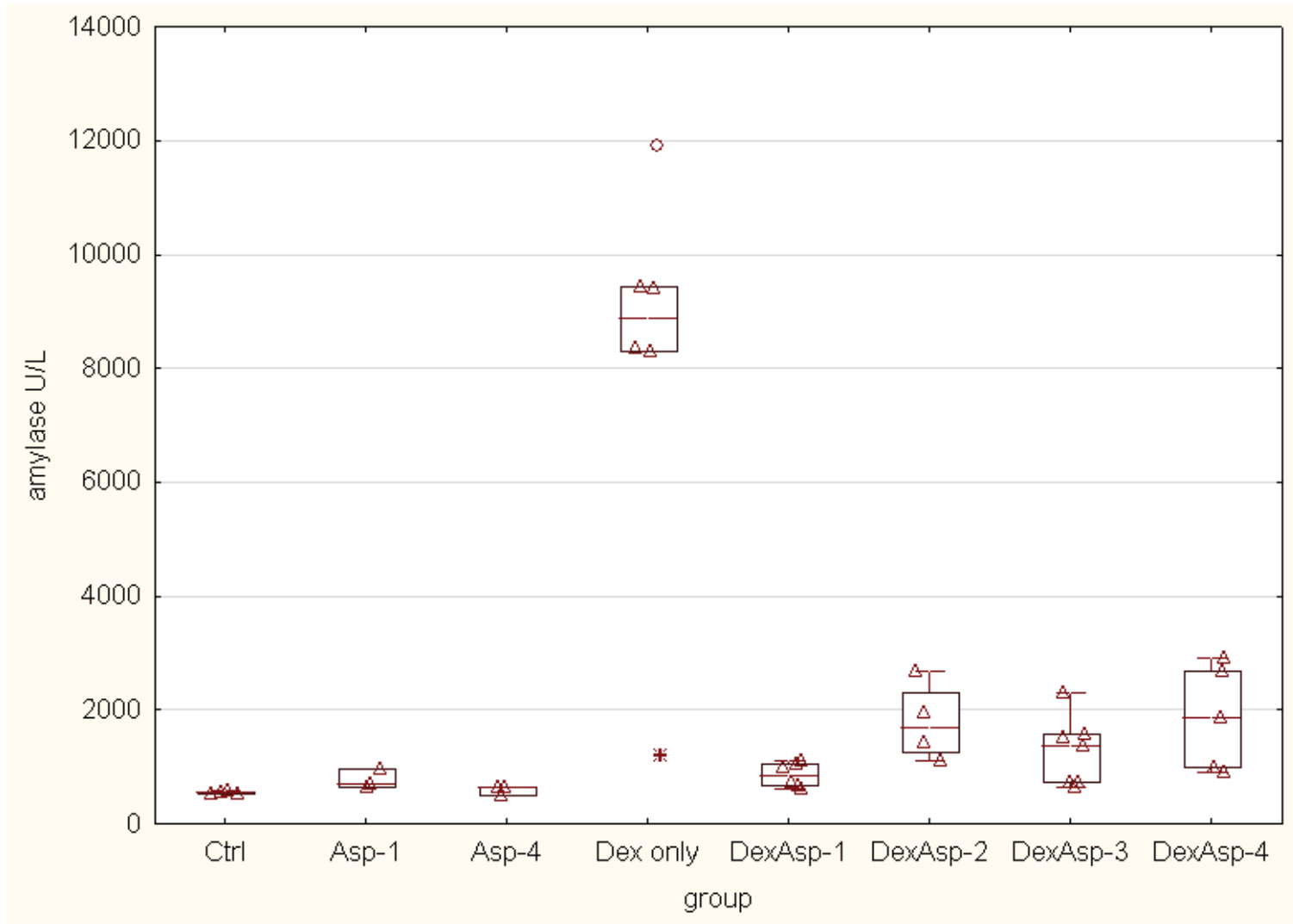

Figure B-9. Mouse plasma amylase level after treatment with dexamethasone and asparaginase

Plasma amylase level was induced by dexamethasone treatment and suppressed by asparaginase treatment. Mice received dexamethasone (Dex; $4 \mathrm{mg} / \mathrm{L}$ in drinking water) for 2 weeks, or dexamethasone and PEG-asparaginase (Asp; $1500 \mathrm{IU} / \mathrm{kg}$ i.p.) at 3.5 day intervals for 1-4 doses, or PEG-asparaginase alone for 1 or 4 doses. Samples were collected at the end of week 2 ( 3.5 days from last asparaginase administration). The reference range of plasma amylase level (salivary and pancreatic) is $602-2311 \mathrm{U} / \mathrm{L}$ in mouse. 
Figure B-10. Mouse plasma lipase level after treatment with dexamethasone and asparaginase

(A) Plasma lipase level was initially inhibited after asparaginase treatment, but then rebound over time. Mice received dexamethasone (Dex; $4 \mathrm{mg} / \mathrm{L}$ in drinking water) for 2 weeks, or dexamethasone and PEG-asparaginase (Asp; $1500 \mathrm{IU} / \mathrm{kg}$ i.p.) at 3.5 day intervals for 1-4 doses, or PEG-asparaginase alone for 1 or 4 doses. Samples were collected at the end of week 2 (3.5 days from last asparaginase administration). (B) Lipase was directly associated with triglyceride $\left(P=9 \times 10^{-5}\right)$. 

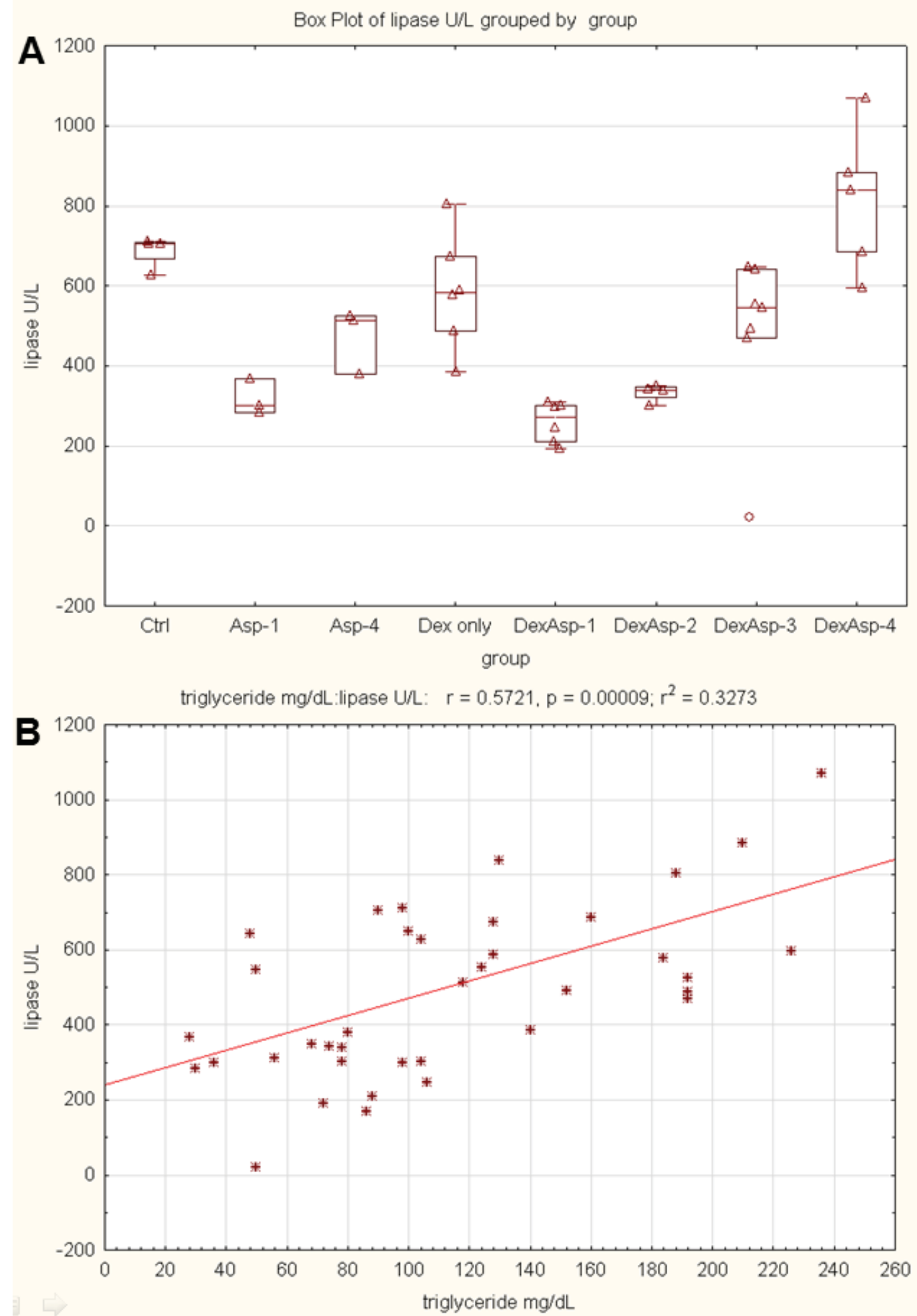


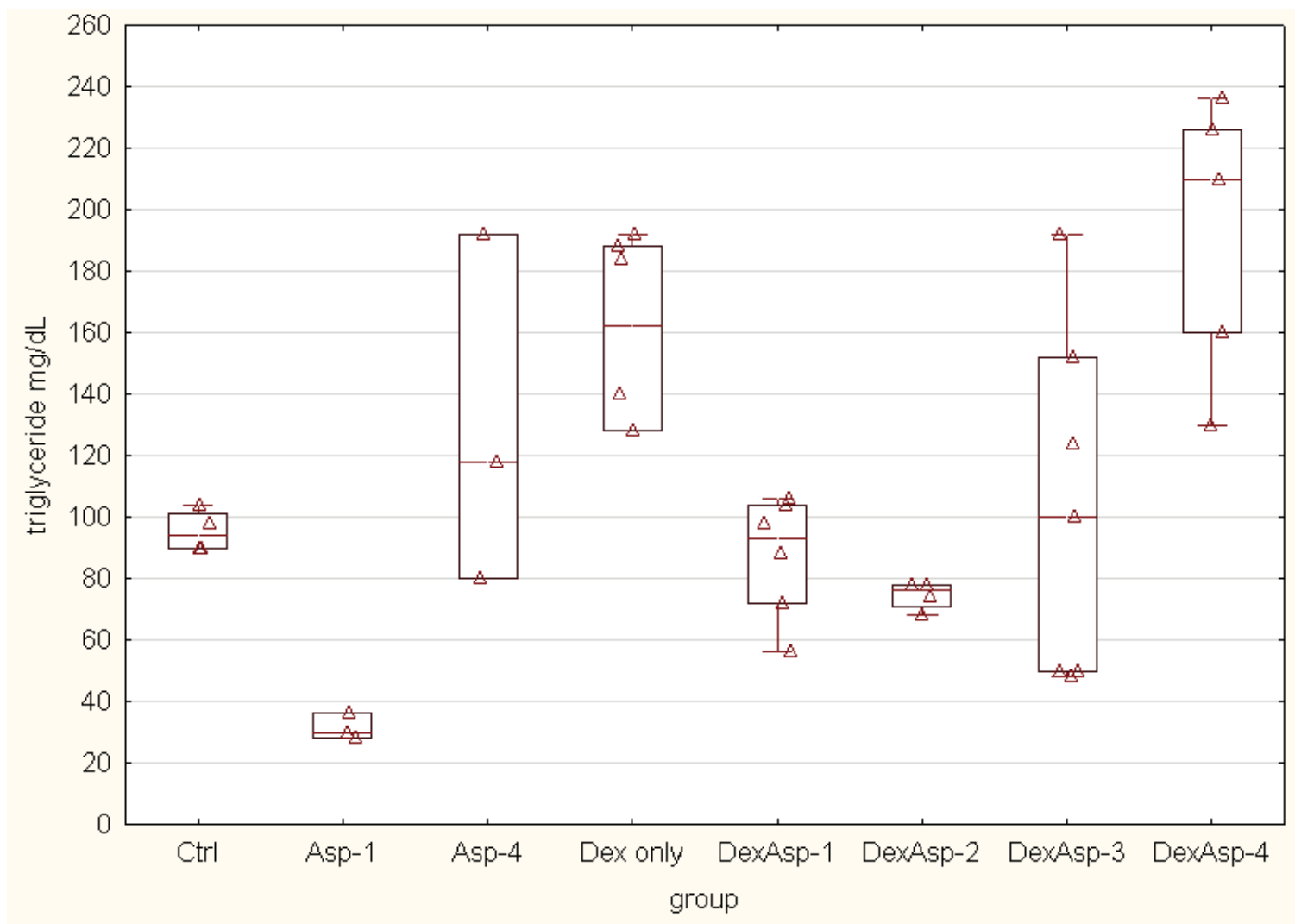

Figure B-11. Mouse plasma triglyceride level after treatment with dexamethasone and asparaginase

Plasma triglyceride level was initially inhibited by asparaginase during the first week, but then increased to a level higher than normal range (reference range 55-144 mg/dl). Mice received dexamethasone (Dex; $4 \mathrm{mg} / \mathrm{L}$ in drinking water) for 2 weeks, or dexamethasone and PEG-asparaginase (Asp; $1500 \mathrm{IU} / \mathrm{kg}$ i.p.) at 3.5 day intervals for 1-4 doses, or PEGasparaginase alone for 1 or 4 doses. Samples were collected at the end of week 2 (3.5 days from last asparaginase administration). 
Figure B-12. Mouse plasma albumin level after treatment with dexamethasone and asparaginase

(A) Plasma albumin level was similar in all groups (Kruskal-Wallis $P=0.10$ ). Mice received dexamethasone (Dex; $4 \mathrm{mg} / \mathrm{L}$ in drinking water) for 2 weeks, or dexamethasone and PEG-asparaginase (Asp; $1500 \mathrm{IU} / \mathrm{kg}$ i.p.) at 3.5 day intervals for 1-4 doses, or PEGasparaginase alone for 1 or 4 doses. Samples were collected at the end of week 2 (3.5 days from last asparaginase administration). (B) No significant change in plasma albumin level with increased asparaginase activity $(P=0.9)$. 

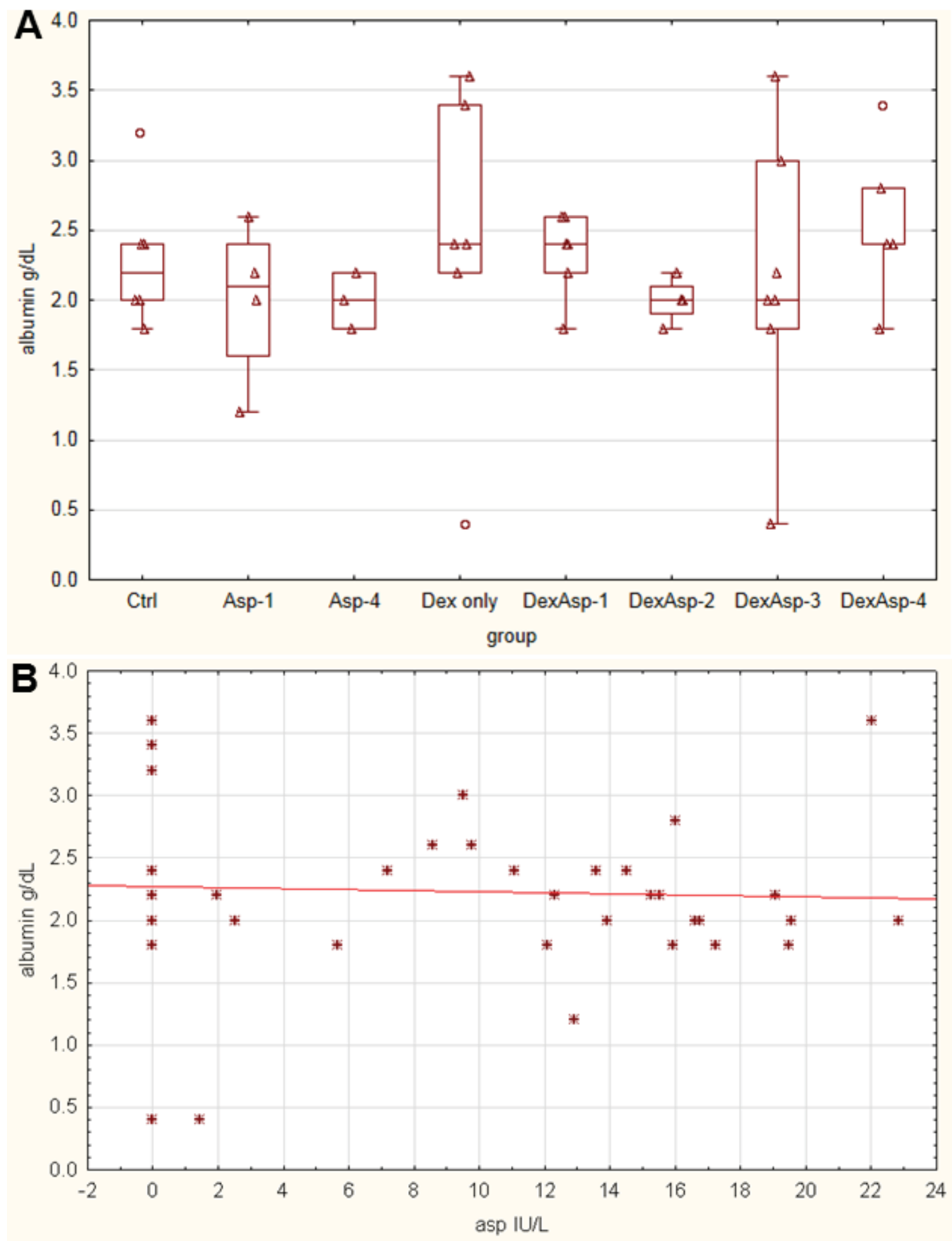


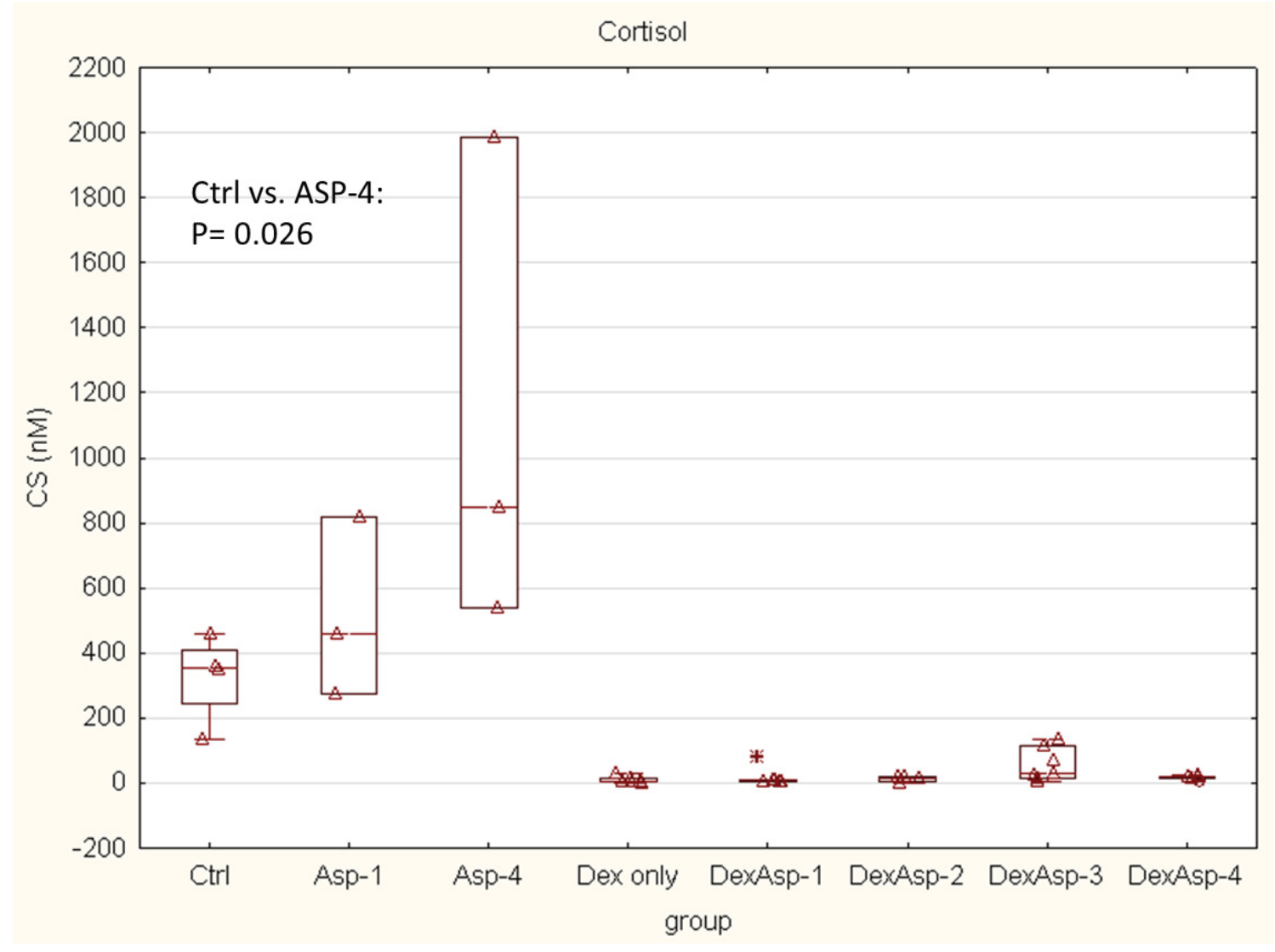

Figure B-13. Mouse plasma cortisol level after treatment with dexamethasone and asparaginase

Plasma cortisol level was induced by asparaginase treatment ( $P=0.026$ after 4 injections) and suppressed by dexamethasone. Mice received dexamethasone (Dex; $4 \mathrm{mg} / \mathrm{L}$ in drinking water) for 2 weeks, or dexamethasone and PEG-asparaginase (Asp; $1500 \mathrm{IU} / \mathrm{kg}$ i.p.) at 3.5 day intervals for 1-4 doses, or PEG-asparaginase alone for 1 or 4 doses.

Samples were collected at the end of week 2 (3.5 days from last asparaginase administration). 
Table B-3. Inhibition of plasma protein level in human and mouse by asparaginase treatment

\begin{tabular}{|c|c|c|c|c|c|c|c|}
\hline Protein & Data source & Inhibition \% & $\begin{array}{c}\text { Nof } \mathbf{A S N} / \\
\text { total } \mathbf{A A}\end{array}$ & ASN $\%$ & $\begin{array}{c}\text { N of GLN/ } \\
\text { total AA }\end{array}$ & GLN\% & $\begin{array}{c}\text { ASN+ } \\
\text { GLN\% }\end{array}$ \\
\hline \multicolumn{8}{|l|}{ Human } \\
\hline Prealbumin & Minowa et al ${ }^{62}$ & $45 \%$ & $4 / 176$ & $2.3 \%$ & $4 / 176$ & $2.3 \%$ & $4.6 \%$ \\
\hline Albumin & Haskell et $\mathrm{al}^{200}$ & $27 \%$ & $17 / 609$ & $2.8 \%$ & $20 / 609$ & $3.3 \%$ & $6.1 \%$ \\
\hline Protein $\mathrm{C}$ & $\begin{array}{l}\text { Saito et al } \\
\text { Appel et } \mathrm{al}^{148}\end{array}$ & $\begin{array}{l}20 \% \\
44 \%\end{array}$ & $13 / 461$ & $2.8 \%$ & $15 / 461$ & $3.3 \%$ & $6.1 \%$ \\
\hline Retinol-binding protein & Minowa et al ${ }^{62}$ & $40 \%$ & $9 / 201$ & $4.5 \%$ & $8 / 201$ & $4.0 \%$ & $8.5 \%$ \\
\hline Transferrin & Minowa et al ${ }^{62}$ & $48 \%$ & $34 / 698$ & $4.9 \%$ & $17 / 698$ & $2.4 \%$ & $7.3 \%$ \\
\hline Plasminogen & $\begin{array}{l}\text { Appel et } \mathrm{al}^{201} \\
\text { Saito et al }\end{array}$ & $\begin{array}{l}39 \% \\
58 \%\end{array}$ & $41 / 810$ & $5.1 \%$ & $31 / 810$ & $3.8 \%$ & $8.9 \%$ \\
\hline Antithrombin & $\begin{array}{c}\text { Merlen et } \mathrm{al}^{202} \\
\text { Hunault-Berger et } \mathrm{al}^{203} \\
\text { Saito et } \mathrm{al}^{148}\end{array}$ & $\begin{array}{l}43 \% \\
50 \% \\
51 \%\end{array}$ & $24 / 464$ & $5.2 \%$ & $13 / 464$ & $2.8 \%$ & $8.0 \%$ \\
\hline Fibrinogen & $\begin{array}{c}\text { Merlen et } \mathrm{al}^{202} \\
\text { Hunault-Berger et } \mathrm{al}^{203} \\
\text { Saito et } \mathrm{al}^{148}\end{array}$ & $\begin{array}{l}51 \% \\
52 \% \\
63 \%\end{array}$ & $11 / 211$ & $5.2 \%$ & $6 / 211$ & $2.8 \%$ & $8.0 \%$ \\
\hline Protein S & Appel et $\mathrm{al}^{201}$ & $55 \%$ & $44 / 676$ & $6.5 \%$ & $25 / 676$ & $3.7 \%$ & $10.2 \%$ \\
\hline
\end{tabular}


Table B-3. (Continued)

\begin{tabular}{|c|c|c|c|c|c|c|c|}
\hline Protein & Data source & Inhibition\% & $\begin{array}{c}\text { Nof ASN/ } \\
\text { total AA }\end{array}$ & ASN\% & $\begin{array}{c}\text { N of GLN/ } \\
\text { total AA }\end{array}$ & GLN\% & $\begin{array}{c}\text { ASN+ } \\
\text { GLN\% }\end{array}$ \\
\hline \multicolumn{8}{|l|}{ Mouse } \\
\hline Albumin & Mouse PK experiment & $18 \% *$ & $19 / 608$ & $3.1 \%$ & $23 / 608$ & $3.8 \%$ & $6.9 \%$ \\
\hline Lipase & Mouse PK experiment & $56 \% *$ & $32 / 465$ & $6.9 \%$ & $18 / 465$ & $3.9 \%$ & $10.8 \%$ \\
\hline Amylase & Mouse PK experiment & $89 \% *$ & $47 / 508$ & $9.3 \%$ & $13 / 508$ & $2.6 \%$ & $11.9 \%$ \\
\hline
\end{tabular}

Data were taken from mouse PK experiment (Figure 3-1) and literatures ${ }^{62,148,200-203}$ and were sorted by the content of asparagine (ASN) residues in the protein. The percentage of inhibition was calculated using the median or mean values of mice who did vs. did not receive asparaginase or of patients pre- vs. post-asparaginase treatment. Glucocorticoids were given concurrently. *Comparing the protein level of dexamethasone alone ( $4 \mathrm{mg} / \mathrm{L}$ for 2 weeks) group vs. the lowest protein level of any dexamethasone and asparaginase (1200 IU/kg i.p. at day 10.5) group. GLN, glutamine; AA, amino acid. 


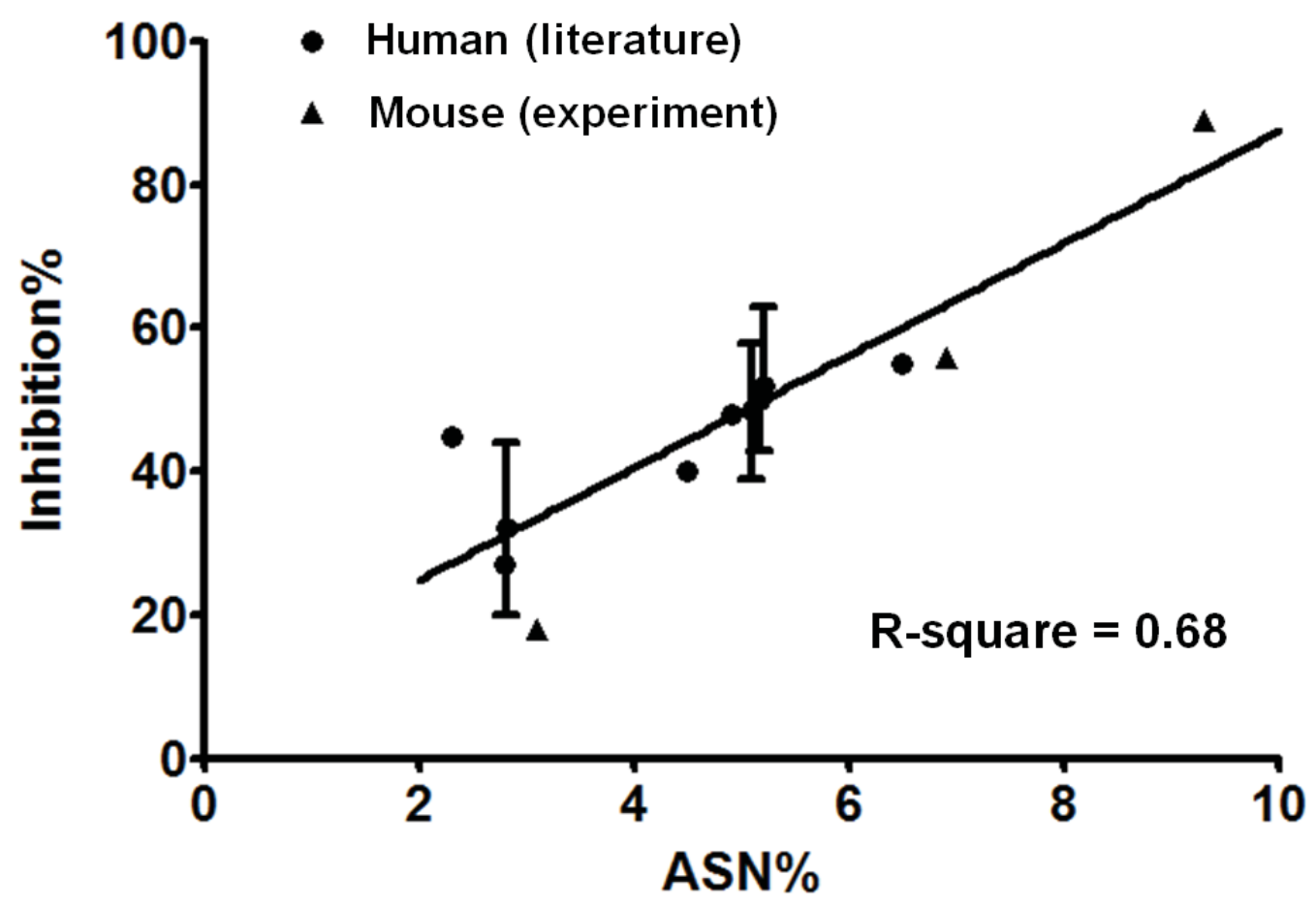

Figure B-14. Correlation between asparagine content in the protein and theextent of inhibition by asparaginase treatment

Data were taken from mouse PK experiment (Figure 3-1) and literatures ${ }^{62,148,200-203}$ (see Table B-3). The percentage of inhibition was plotted against the percentage of asparagine (ASN) residues in the protein. Median, range and linear regression line are shown. 


\section{APPENDIX C. SUPPLEMENTARY INFORMATION FOR CHAPTER 4}

\section{Deep Sequencing of Selected Genes}

To identify rare and low-frequency variants in genes of interest for ALL-related phenotypes, we sequenced 283 genes. The genes were selected for sequencing based on their prior association with ALL phenotypes of interest (e.g. relapse, drug sensitivity, pharmacokinetics, leukemia risk, and adverse effects). Of these, 42 were putatively pancreatitis-associated genes: 34 were top genes from preliminary results of the current GWAS, and 8 were candidate genes associated with pancreatitis in other settings; Table 4-3). Illumina dual-indexed libraries were constructed using germline DNA from 4217 children in the cohort (including 97 cases with pancreatitis) and 162 in the case-control group (including 47 cases). These libraries were pooled in sets of 96 and hybridized with the Roche NimbleGen SeqCap EZ custom probes to capture the selected gene regions. Targeted regions for sequencing include all coding regions and putative functional regions within $10 \mathrm{~K}$ base pairs of the transcripts including promoters and enhancers as predicted according to ENCODE/Roadmap Epigenomics data. ${ }^{204}$ In addition, we also sequenced the putative functional regions within the linkage disequilibrium block defined by the top GWAS SNPs. The titer of samples was determined by PCR, and the flow cell was sequenced on the Illumina HiSeq 2000 as a paired-end, multiplexed, $2 \times 101 \mathrm{bp}$ run. The pooled sample was then demultiplexed with coverage of $>20 \times$ depth across $>90 \%$ of the targeted regions. Burrows-Wheeler Alignment was performed on the sequence reads and GATK pipeline version 3.1 was used to call the genetic variants. The concordance between deep sequencing and arrays was greater than $98 \%$ for 3635 of the 3639 SNPs on both platforms.

\section{SNP-based and Gene-based Analysis of Variants Identified by Deep Sequencing}

After excluding the SNPs with a call rate less than 95\% and a Variant Quality Score Recalibration (VQSR) score greater than 99.5\%, we interrogated 9229 SNPs in 283 genes. Genotype calls were coded as 0,1 or 2 for the number of $\mathrm{B}$ alleles (AA, AB or $\mathrm{BB}$ ) assuming an additive genetic model. For patients with deep sequencing data in the cohort $(n=4217)$, GWAS was performed using a Cox proportional hazard model considering the onset time of pancreatitis (time-dependent GWAS). Covariates included age (continuous variable in years) and genetic ancestry (continuous variable as percentages of European, African, Asian and Native American ancestries) and treatment regimen (asparaginase dose $\leq 120000 \mathrm{U} / \mathrm{m}^{2}$ vs. $\geq 240000 \mathrm{U} / \mathrm{m}^{2}$ ) coded as a categorical variable. For patients with pancreatitis, their time-at-risk was censored at the date of first episode of pancreatitis; for those without pancreatitis, their time-at-risk was censored at off-study date. In the case-control group $(n=162)$, cases and controls were matched for age and treatment regimen, and GWAS was performed using logistic regression, adjusted for genetic ancestry only (time-independent GWAS). 
SNPs were assigned to genes according to Refseq boundaries downloaded from UCSC Genome Browser (hg19 assembly). For the 283 genes sequenced, we applied sequence kernel association test ${ }^{161}$ (SKAT) to test for gene level significance for the effects of nonsense variants. For time dependent analysis, we first obtained Martingale residuals as a continuous phenotype in the SKAT analysis after applying Cox proportional hazard model using demographic and clinical covariates, including age, genetic ancestry, and treatment regimen. 


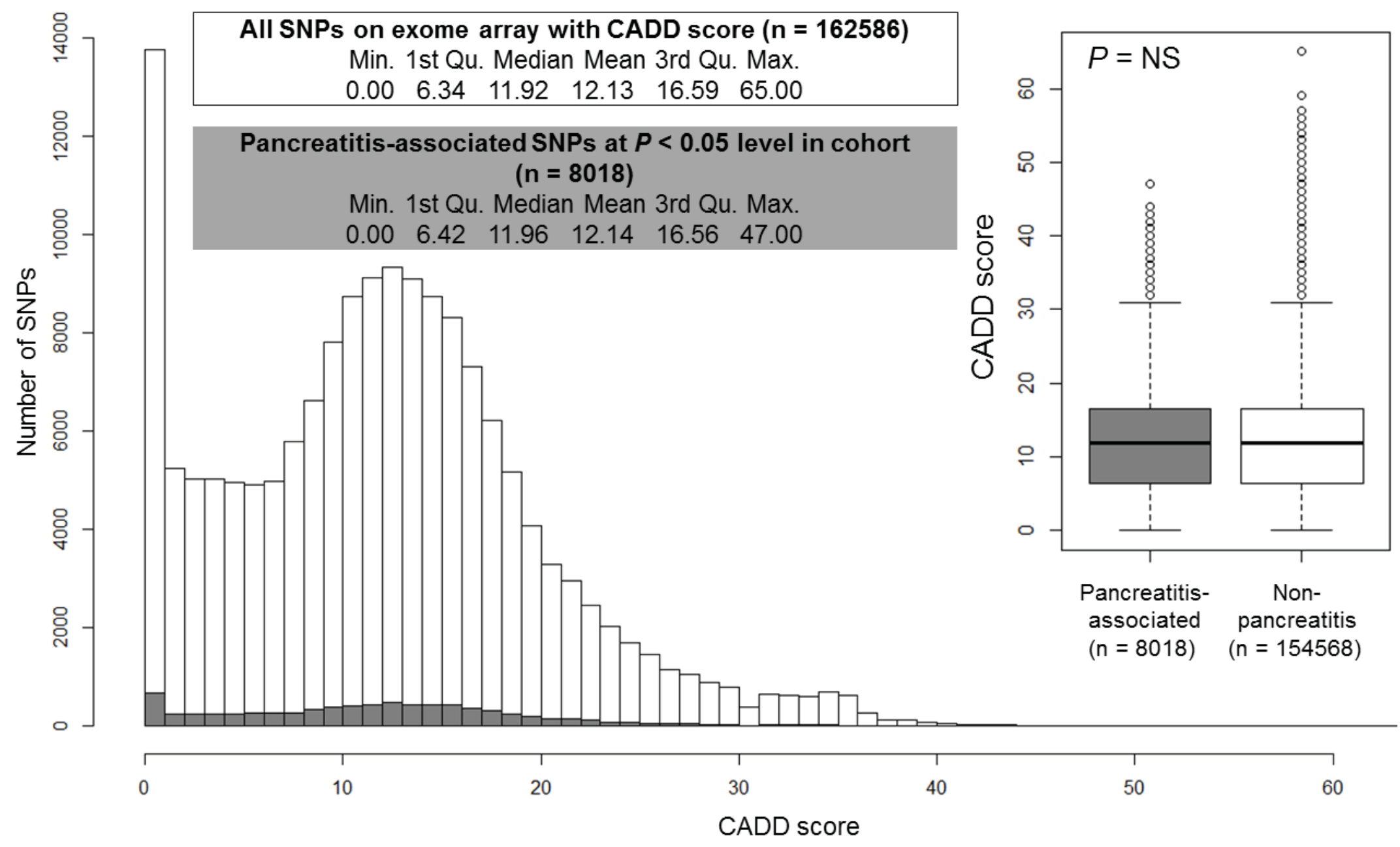

Figure C-1. CADD score in all SNPs on exome array $(n=162586)$ and pancreatitis-associated $\mathrm{SNPs}(P<0.05 ; n=$ 8018)

Distribution of CADD score of SNPs associated $(P<0.05)$ with pancreatitis $(\mathrm{n}=8018$, gray bar) in the cohort vs. all SNPs on exome array with a CADD score $(n=162586$, clear bar). The range, median, mean and quartile values are shown. Inset graph shows similar CADD score of pancreatitis-associated vs. non-pancreatitis SNPs. NS, not significant. 
Table C-1. Top 10 SNPs from genome-wide analysis of nonsense SNPs; $\mathbf{n}=\mathbf{2 , 3 5 7}$ SNPs

\begin{tabular}{|c|c|c|c|c|c|c|c|c|c|c|c|c|c|}
\hline \multirow[b]{2}{*}{ SNP } & \multirow[b]{2}{*}{ Gene } & \multirow{2}{*}{\multicolumn{2}{|c|}{ Chr Location $^{\mathrm{a}}$}} & \multirow[b]{2}{*}{$\begin{array}{c}\text { Major } \\
\text { allele/ } \\
\text { risk } \\
\text { allele }\end{array}$} & \multirow[b]{2}{*}{$\begin{array}{l}\text { Population } \\
\text { RAF\% (n) }\end{array}$} & \multicolumn{4}{|c|}{ Cohort $(n=3256)$} & \multicolumn{4}{|c|}{ Case/control $(n=213)$} \\
\hline & & & & & & $\begin{array}{c}\text { RAF\% in } \\
\text { patients } \\
\text { with AP } \\
(n=95)\end{array}$ & $\begin{array}{c}\text { RAF\% in } \\
\text { patients } \\
\text { without AP } \\
(n=3161)\end{array}$ & $\begin{array}{l}\text { Coeff- } \\
\text { icient }^{c}\end{array}$ & $P^{\mathbf{c}}$ & $\begin{array}{l}\text { RAF\% } \\
\text { in cases } \\
(n=71)\end{array}$ & $\begin{array}{c}\text { RAF\% } \\
\text { in } \\
\text { controls } \\
(n=142)\end{array}$ & $\begin{array}{l}\text { Coeff- } \\
\text { icient }^{d}\end{array}$ & $P^{d}$ \\
\hline rs199695765 & CPA2 & 7 & 129696084 & $\mathrm{C} / \mathrm{T}$ & $0.009(11)$ & 0.5 & 0 & 6.6 & $1.5 \times 10^{-8}$ & 0.8 & 0 & 15.8 & $\overline{0.08}$ \\
\hline rs 144556038 & ZNF701 & 19 & 57777659 & $\mathrm{C} / \mathrm{T}$ & $0.04(46)$ & 1.1 & 0.03 & 3.6 & $2.0 \times 10^{-6}$ & 0.8 & 0 & 15.1 & 0.17 \\
\hline rs 116191233 & C17orf66 & 17 & 31215928 & $\mathrm{G} / \mathrm{A}$ & & 0.5 & 0.02 & 3.3 & 0.001 & 0.8 & 0 & 15.5 & 0.12 \\
\hline rs79537388 & C9orf117 & 9 & 129515133 & $\mathrm{C} / \mathrm{T}$ & $0.2(171)$ & 1.6 & 0.2 & 1.7 & 0.005 & 0.8 & 0 & 15.3 & 0.17 \\
\hline rs 113207856 & $S L C 26 A 10$ & 12 & 56304127 & $\mathrm{G} / \mathrm{A}$ & $0.4(515)$ & 1.6 & 0.4 & 1.6 & 0.006 & 1.7 & 0 & 16.3 & 0.04 \\
\hline rs78283108 & SERPINA9 & 14 & 94005381 & $\mathrm{G} / \mathrm{A}$ & $0.6(732)$ & 2.7 & 0.7 & 1.2 & 0.015 & 0.8 & 0 & 15.1 & 0.17 \\
\hline rs1023840 & HEATR7B2 & 25 & 41097472 & $\mathrm{C} / \mathrm{T}$ & $22.6(23995)$ & 31.5 & 21.4 & 0.4 & 0.016 & 24.6 & 23.6 & 0.03 & 0.90 \\
\hline rs41463245 & CCL26 & 7 & 75239199 & $\mathrm{C} / \mathrm{T}$ & $0.9(1053)$ & 2.2 & 0.8 & 1.2 & 0.019 & 1.7 & 0 & 0.09 & 0.92 \\
\hline rs67047829 & ERV3-1 & 7 & 64090173 & $\mathrm{G} / \mathrm{A}$ & 10.8 (12129) & 15.8 & 9.4 & 0.5 & 0.022 & 14.4 & 9.4 & 4.7 & 0.21 \\
\hline rs 146053779 & $G C K R$ & 2 & 27598876 & $\mathrm{C} / \mathrm{T}$ & 0.09 (111) & 1.1 & 0.3 & 1.6 & 0.024 & 0.8 & 0 & 15.5 & 0.75 \\
\hline
\end{tabular}

The SNPs were rank ordered by $\mathrm{P}$ value in the cohort study, and then filtered by the direction in the case-control study.

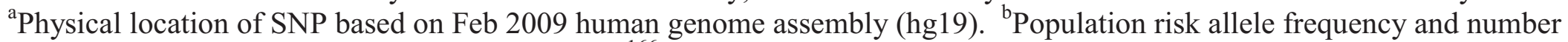
of carriers of the SNP based on the ExAC data set ${ }^{166}$ of 61486 unrelated individuals of European, African, Latino and Asian origins. ${ }^{\mathrm{C}}$ Coefficient and $\mathrm{P}$ value of risk alleles were estimated using Cox proportional hazard model. ${ }^{\mathrm{d}}$ Coefficient and $\mathrm{P}$ value of risk alleles were estimated using logistic regression model. Chr, chromosome. AP, acute pancreatitis. RAF, risk allele frequency. 
Table C-2. Top 10 SNPs from genome-wide analysis of missense SNPs (excluding nonsense SNPs); $n=144,349$ SNPs

\begin{tabular}{|c|c|c|c|c|c|c|c|c|c|c|c|c|c|}
\hline \multirow[b]{2}{*}{ SNP } & \multirow[b]{2}{*}{ Gene } & \multirow[b]{2}{*}{ Chr } & \multirow[b]{2}{*}{ Location $^{\mathrm{a}}$} & \multirow[b]{2}{*}{$\begin{array}{c}\text { Major } \\
\text { allele/ } \\
\text { risk } \\
\text { allele }\end{array}$} & \multirow[b]{2}{*}{$\begin{array}{l}\text { Population } \\
\text { RAF\% (n) }\end{array}$} & \multicolumn{4}{|c|}{ Cohort $(n=3256)$} & \multicolumn{4}{|c|}{ Case/control $(n=213)$} \\
\hline & & & & & & $\begin{array}{c}\text { RAF\% in } \\
\text { patients } \\
\text { with AP } \\
(n=95)\end{array}$ & $\begin{array}{c}\mathrm{RAF} \% \text { in } \\
\text { patients } \\
\text { without AP } \\
(\mathrm{n}=3161)\end{array}$ & $\begin{array}{l}\text { Coeff- } \\
\text { icient }^{c}\end{array}$ & $P^{\mathrm{c}}$ & $\begin{array}{l}\text { RAF\% } \\
\text { in cases } \\
(n=71)\end{array}$ & $\begin{array}{l}\text { RAF\% } \\
\text { in } \\
\text { controls } \\
(\mathrm{n}=142)\end{array}$ & $\begin{array}{l}\text { Coeff- } \\
\text { icient }^{d}\end{array}$ & $P^{d}$ \\
\hline rs112141546 & ADAMTS18 & 16 & 77401515 & $\mathrm{C} / \mathrm{T}$ & $0.2(225)$ & 0.2 & 0.03 & 3.3 & $4.2 \times 10^{-10}$ & 0.8 & 0 & 14.9 & 0.28 \\
\hline rs45461499 & $C D C 20$ & 1 & 43828736 & G/A & $0.05(65)$ & 1.1 & 0.2 & 4.6 & $3.6 \times 10^{-9}$ & 0.8 & 0.4 & 1.1 & 0.46 \\
\hline rs200495769 & PDLIM5 & 4 & 95494528 & $\mathrm{C} / \mathrm{T}$ & $0.01(12)$ & 0.5 & 0.02 & 5.7 & $2.9 \times 10^{-7}$ & 0.8 & 0.4 & 1.0 & 0.51 \\
\hline rs141708090 & C3orf 20 & 3 & 14803109 & $\mathrm{G} / \mathrm{T}$ & $0.2(243)$ & 1.1 & 0.02 & 3.6 & $5.7 \times 10^{-7}$ & 0.8 & 0 & 15.3 & 0.14 \\
\hline $\begin{array}{c}19: 49564629 \\
\mathrm{C} / \mathrm{T}\end{array}$ & NTF4 & 19 & 49564629 & $\mathrm{C} / \mathrm{T}$ & $0.01(16)$ & 0.5 & 0 & 5.1 & $7.0 \times 10^{-7}$ & 0.8 & 0 & 15.3 & 0.14 \\
\hline rs201734206 & C8orf84 & 8 & 73984100 & $\mathrm{C} / \mathrm{T}$ & $0.04(46)$ & 0.5 & 0 & 4.9 & $2.0 \times 10^{-6}$ & 0.8 & 0 & 15.3 & 0.14 \\
\hline rs202124287 & $N E B$ & 2 & 152534216 & $\mathrm{C} / \mathrm{T}$ & $0.06(74)$ & 0.5 & 0 & 4.8 & $3.8 \times 10^{-6}$ & 0.8 & 0 & 15.4 & 0.13 \\
\hline rs143348702 & MICAL1 & 6 & 109770636 & $\mathrm{~T} / \mathrm{C}$ & $0.02(27)$ & 0.5 & 0 & 4.6 & $9.9 \times 10^{-6}$ & 0.8 & 0 & 16.0 & 0.07 \\
\hline rs147501499 & MYOM2 & 8 & 2092718 & $\mathrm{C} / \mathrm{T}$ & $0.2(266)$ & 0.5 & 0 & 4.5 & $1.2 \times 10^{-5}$ & 0.8 & 0 & 14.6 & 0.24 \\
\hline rs 141452852 & $T A S 2 R 16$ & 7 & 122635645 & $\mathrm{~A} / \mathrm{G}$ & $0.02(24)$ & 1.1 & 0.05 & 3.1 & $1.5 \times 10^{-5}$ & 0.8 & 0 & 15.2 & 0.15 \\
\hline
\end{tabular}

The SNPs were rank ordered by $\mathrm{P}$ value in the cohort study, and then filtered by the direction in the case-control study. ${ }^{a}$ Physical location of SNP based on Feb 2009 human genome assembly (hg19). ${ }^{b}$ Population risk allele frequency and number of carriers of the SNP based on the ExAC data set ${ }^{166}$ of 61486 unrelated individuals of European, African, Latino and Asian origins. ${ }^{\mathrm{C}}$ Coefficient and $\mathrm{P}$ value of risk alleles were estimated using Cox proportional hazard model. ${ }^{\mathrm{d}}$ Coefficient and P value of risk alleles were estimated using logistic regression model. Chr, chromosome. AP, acute pancreatitis. RAF, risk allele frequency. 
Table C-3. Top 10 SNPs from genome-wide analysis of synonymous and non-coding SNPs; n = 774,238 SNPs

\begin{tabular}{|c|c|c|c|c|c|c|c|c|c|c|c|c|c|c|}
\hline \multirow[b]{2}{*}{ SNP } & \multirow[b]{2}{*}{ Gene } & \multirow{2}{*}{\multicolumn{2}{|c|}{ Chr Location ${ }^{\mathrm{a}}$}} & \multirow{2}{*}{\multicolumn{2}{|c|}{$\begin{array}{r}\text { Function Major } \\
\text { allele/ } \\
\text { risk } \\
\text { allele }\end{array}$}} & \multirow[b]{2}{*}{$\begin{array}{c}\text { Popu- } \\
\text { lation } \\
\text { RAF\% } \\
(\mathbf{n})^{\mathbf{b}}\end{array}$} & \multicolumn{4}{|c|}{ Cohort $(n=3256)$} & \multicolumn{4}{|c|}{ Case/ctrl $(n=213)$} \\
\hline & & & & & & & $\begin{array}{l}\text { RAF\% } \\
\text { in } \\
\text { patients } \\
\text { with AP } \\
(n=95)\end{array}$ & $\begin{array}{c}\text { RAF\% in } \\
\text { patients } \\
\text { without } \\
\text { AP } \\
(n=3161)\end{array}$ & $\begin{array}{l}\text { Coeff- } \\
\text { icient }^{\mathrm{c}}\end{array}$ & $P^{\mathrm{c}}$ & $\begin{array}{c}\text { RAF\% } \\
\text { in } \\
\text { cases } \\
(\mathbf{n}= \\
71)\end{array}$ & $\begin{array}{c}\text { RAF\% } \\
\text { in } \\
\text { controls } \\
(n= \\
142)\end{array}$ & $\begin{array}{l}\text { Coeff- } \\
\text { icient }^{d}\end{array}$ & $P^{\mathrm{d}}$ \\
\hline rs112141546 & $H O G A 1$ & 10 & 99349783 & Syn & $\mathrm{C} / \mathrm{T}$ & $0.2(242)$ & 2.2 & 0.03 & 3.5 & $1.1 \times 10^{-6}$ & 0.8 & 0 & 14.9 & 0.15 \\
\hline rs9849262 & FHIT & 3 & 60912089 & Intron & $\mathrm{C} / \mathrm{T}$ & $2.7 *(132)$ & 4.7 & 1.0 & 1.4 & $8.4 \times 10^{-6}$ & 0.7 & 0 & 13.7 & 0.18 \\
\hline rs12589386 & - & 14 & 46293715 & - & $\mathrm{A} / \mathrm{G}$ & $8.3 *(416)$ & 8.5 & 3.7 & 2.8 & $1.8 \times 10^{-5}$ & 9.4 & 3.2 & 1.0 & 0.03 \\
\hline rs 142728074 & SPATA21 & 1 & 16727294 & Syn & $\mathrm{G} / \mathrm{A}$ & $0.07(91)$ & 1.1 & 0.05 & 3.3 & $2.5 \times 10^{-5}$ & 0.8 & 0 & 14.7 & 0.23 \\
\hline rs6721376 & - & & 240102585 & - & $\mathrm{C} / \mathrm{T}$ & $2.9 *(146)$ & 3.8 & 0.9 & 0.2 & $5.0 \times 10^{-5}$ & 2.2 & 0 & 16.7 & 0.02 \\
\hline rs17117423 & - & 14 & 46078262 & - & $\mathrm{C} / \mathrm{T}$ & $9.5 *(476)$ & 9.1 & 4.2 & 0.4 & $5.0 \times 10^{-5}$ & 8.7 & 2.9 & 1.3 & 0.02 \\
\hline rs10979693 & C9orf5 & 9 & 110916644 & Intron & $\mathrm{A} / \mathrm{C}$ & $2.6^{*}(127)$ & 2.1 & 0.4 & 5.8 & $6.4 \times 10^{-5}$ & 0.7 & 0 & 13.7 & 0.39 \\
\hline rs9859201 & FHIT & 3 & 60910412 & Intron & $\mathrm{A} / \mathrm{T}$ & $2.9 *(145)$ & 4.3 & 1.1 & 3.6 & $1.2 \times 10^{-4}$ & 0.7 & 0 & 13.7 & 0.18 \\
\hline rs6724701 & - & 2 & 180968192 & - & $\mathrm{G} / \mathrm{A}$ & $0.9 *(46)$ & 3.1 & 0.6 & 5.1 & $1.4 \times 10^{-4}$ & 2.3 & 0 & 17.0 & 0.01 \\
\hline rs2010463 & MICAL2 & 11 & 12142872 & Intron & $\mathrm{C} / \mathrm{T}$ & $\begin{array}{c}55.8 * \\
(2213) \\
\end{array}$ & 61.1 & 44.7 & 0.6 & $2.0 \times 10^{-4}$ & 54.5 & 34.6 & 0.6 & 0.003 \\
\hline
\end{tabular}

The SNPs were rank ordered by $\mathrm{P}$ value in the cohort study, and then filtered by the direction in the case-control study. If the risk allele frequency is greater than $1 \%$ in the case-controls study, the respective P value should be less than 0.05 . Population risk allele frequency and number of carriers of the SNP based on the ExAC data set ${ }^{166}$ of 61486 unrelated individuals of European, African, Latino and Asian origins, and *population risk allele frequency and number of alleles based on 1000Genome phase 1 population (http://www.1000genomes.org/node/506 [May 2011]) of 1088 individuals. ${ }^{\mathrm{c}}$ Coefficient and P value of risk alleles were estimated using Cox proportional hazard model. ${ }^{\mathrm{d}}$ Coefficient and $\mathrm{P}$ value of risk alleles were estimated using logistic regression model. AP, acute pancreatitis. Chr, chromosome. RAF, risk allele frequency. Syn, synonymous. 
Table C-4. All 380 CPA2 variants identified in the cohort $(n=4217)$ and the case-control group $(n=162)$

\begin{tabular}{|c|c|c|c|c|c|c|c|c|c|c|c|}
\hline \multirow[b]{2}{*}{ Chr:Loc } & \multirow[b]{2}{*}{ RSID } & \multirow[b]{2}{*}{$\begin{array}{l}\text { Loca- } \\
\text { tion }\end{array}$} & \multirow[b]{2}{*}{ Function } & \multicolumn{4}{|c|}{ Cohort $(n=4217)$} & \multicolumn{4}{|c|}{ Case-control $(n=162)$} \\
\hline & & & & $\begin{array}{c}\text { MAF\% in } \\
\text { patients } \\
\text { with AP } \\
(n=97)\end{array}$ & $\begin{array}{l}\text { MAF\% in } \\
\text { patients } \\
\text { without } \\
\text { AP } \\
(n=3220) \\
\end{array}$ & $\begin{array}{l}\text { Coeff- } \\
\text { icient }^{b}\end{array}$ & $P^{\mathbf{b}}$ & $\begin{array}{l}\text { MAF\% } \\
\text { in cases } \\
(n=47)\end{array}$ & $\begin{array}{c}\text { MAF\% in } \\
\text { controls } \\
(n=115)\end{array}$ & $\begin{array}{l}\text { Coeff- } \\
\text { icient }^{c}\end{array}$ & $P^{\mathbf{c}}$ \\
\hline $7: 129912621$ & & Intron & & 0.52 & 0.00 & 6.1 & $5.00 \times 10^{-10}$ & 0.00 & 0.00 & NA & NA \\
\hline 7:129917351 & rs19187532 & Intron & & 0.52 & 0.00 & 6.7 & $6.03 \times 10^{-10}$ & 0.00 & 0.00 & NA & NA \\
\hline $7: 129908848$ & rs199695765 & Exon & $\begin{array}{l}\text { Stop-gain } \\
\text { damaging }\end{array}$ & 0.52 & 0.00 & 6.1 & $1.38 \times 10^{-8}$ & 1.06 & 0.00 & 15.8 & 0.986 \\
\hline $7: 129908689$ & & Intron & & 0.52 & 0.00 & 6.1 & $1.42 \times 10^{-8}$ & 0.00 & 0.00 & NA & NA \\
\hline 7:129929042 & rs7544262 & Intron & & 0.52 & 0.00 & 4.7 & $4.65 \times 10^{-6}$ & 0.00 & 0.00 & NA & NA \\
\hline $7: 129916139$ & & Intron & & 0.52 & 0.00 & 4.6 & $7.17 \times 10^{-6}$ & 0.00 & 0.00 & NA & NA \\
\hline $7: 129908835$ & & Exon & Syn & 0.52 & 0.00 & 3.9 & $1.12 \times 10^{-4}$ & 0.00 & 0.00 & NA & NA \\
\hline $7: 129915385$ & & Intron & & 0.52 & 0.00 & 4.0 & $1.58 \times 10^{-4}$ & 0.00 & 0.00 & NA & NA \\
\hline $7: 129915649$ & & Intron & & 0.52 & 0.00 & 3.5 & $9.56 \times 10^{-4}$ & 0.00 & 0.00 & NA & NA \\
\hline $7: 129915690$ & & Intron & & 0.52 & 0.00 & 3.3 & $1.28 \times 10^{-3}$ & 0.00 & 0.00 & NA & NA \\
\hline $7: 129915313$ & rs18247836 & Intron & & 0.52 & 0.01 & 2.9 & $4.21 \times 10^{-3}$ & 0.00 & 0.00 & NA & NA \\
\hline $7: 129915817$ & & Intron & & 0.52 & 0.04 & 2.5 & $1.37 \times 10^{-2}$ & 0.00 & 0.00 & NA & NA \\
\hline $7: 129913443$ & & Intron & & 0.52 & 0.01 & 2.3 & $2.05 \times 10^{-2}$ & 0.00 & 0.00 & NA & NA \\
\hline 7:129929468 & rs 146796996 & Exon & $\begin{array}{l}\text { Nonsyn } \\
\text { damaging }\end{array}$ & 0.52 & 0.06 & 2.2 & $2.68 \times 10^{-2}$ & 0.00 & 0.00 & NA & NA \\
\hline $7: 129912224$ & rs15176341 & Intron & & 0.52 & 0.02 & 2.1 & $3.68 \times 10^{-2}$ & 0.00 & 0.00 & NA & NA \\
\hline 7:129917748 & rs139875564 & Exon & $\begin{array}{c}\text { Nonsyn } \\
\text { damaging }\end{array}$ & 0.52 & 0.02 & 2.1 & $3.68 \times 10^{-2}$ & 0.00 & 0.00 & NA & NA \\
\hline $7: 129916325$ & rs4127417 & Intron & & 21.35 & 19.88 & 0.3 & $5.24 \times 10^{-2}$ & 11.70 & 18.26 & -0.4 & 0.342 \\
\hline $7: 129916008$ & rs73146775 & Intron & & 21.13 & 19.81 & 0.3 & $5.74 \times 10^{-2}$ & 11.70 & 18.26 & -0.4 & 0.342 \\
\hline $7: 129916960$ & rs1198355 & Intron & & 21.13 & 19.82 & 0.3 & $5.77 \times 10^{-2}$ & 11.70 & 18.26 & -0.4 & 0.342 \\
\hline $7: 129915538$ & rs11971847 & Intron & & 21.13 & 19.83 & 0.3 & $5.89 \times 10^{-2}$ & 11.70 & 18.26 & -0.4 & 0.342 \\
\hline 7:129919105 & rs12536877 & Intron & & 21.13 & 19.83 & 0.3 & $5.95 \times 10^{-2}$ & 11.70 & 18.26 & -0.4 & 0.342 \\
\hline 7:129913198 & rs1197851 & Intron & & 20.10 & 18.81 & 0.3 & $6.43 \times 10^{-2}$ & 10.64 & 18.26 & -0.5 & 0.243 \\
\hline
\end{tabular}


Table C-4. (Continued)

\begin{tabular}{|c|c|c|c|c|c|c|c|c|c|c|c|}
\hline \multirow[b]{2}{*}{ Chr:Loc ${ }^{a}$} & \multirow[b]{2}{*}{ RSID } & \multirow[b]{2}{*}{$\begin{array}{c}\text { Loca- } \\
\text { tion }\end{array}$} & \multirow[b]{2}{*}{ Function } & \multicolumn{4}{|c|}{ Cohort $(n=4217)$} & \multicolumn{4}{|c|}{ Case-control $(n=162)$} \\
\hline & & & & $\begin{array}{c}\text { MAF\% in } \\
\text { patients } \\
\text { with AP } \\
(n=97)\end{array}$ & $\begin{array}{c}\text { MAF\% in } \\
\text { patients } \\
\text { without } \\
\text { AP } \\
(n=3220)\end{array}$ & $\begin{array}{l}\text { Coeff- } \\
\text { icient }^{b}\end{array}$ & $P^{\mathbf{b}}$ & $\begin{array}{l}\text { MAF\% } \\
\text { in cases } \\
(n=47)\end{array}$ & $\begin{array}{c}\text { MAF\% in } \\
\text { controls } \\
(n=115)\end{array}$ & $\begin{array}{l}\text { Coeff- } \\
\text { icient }^{c}\end{array}$ & $P^{\mathbf{c}}$ \\
\hline $7: 129912349$ & rs115716317 & Intron & & 1.03 & 0.22 & 1.4 & $6.87 \times 10^{-2}$ & 0.00 & 0.00 & $\mathrm{NA}$ & NA \\
\hline 7:129913993 & rs 14977797 & Intron & & 1.03 & 0.41 & 1.3 & $7.05 \times 10^{-2}$ & 0.00 & 0.43 & -13.3 & 0.988 \\
\hline $7: 129915816$ & & Intron & & 0.52 & 0.11 & 1.8 & $7.20 \times 10^{-2}$ & 0.00 & 0.00 & NA & NA \\
\hline $7: 129917148$ & rs 14223236 & Intron & & 1.03 & 0.39 & 1.2 & $1.07 \times 10^{-1}$ & 0.00 & 0.43 & -13.3 & 0.988 \\
\hline $7: 129929673$ & rs73146781 & $\begin{array}{l}\text { Down- } \\
\text { stream }\end{array}$ & & 20.10 & 19.71 & 0.3 & $1.28 \times 10^{-1}$ & 12.77 & 16.96 & -0.2 & 0.656 \\
\hline $7: 129913520$ & rs125753 & Intron & & 28.80 & 31.15 & -0.3 & $1.31 \times 10^{-1}$ & 41.11 & 37.16 & 0.3 & 0.539 \\
\hline $7: 129913510$ & & Intron & & 0.62 & 0.16 & 1.4 & $1.77 \times 10^{-1}$ & 0.00 & 0.00 & NA & NA \\
\hline $7: 129912168$ & rs6467292 & Intron & & 28.87 & 26.58 & 0.2 & $2.18 \times 10^{-1}$ & 13.83 & 23.48 & -0.5 & 0.147 \\
\hline $7: 129912145$ & rs111673838 & Intron & & 28.87 & 26.60 & 0.2 & $2.22 \times 10^{-1}$ & 13.83 & 23.48 & -0.5 & 0.147 \\
\hline $7: 129915016$ & rs61731658 & Exon & Nonsyn & 2.06 & 0.68 & 0.7 & $2.23 \times 10^{-1}$ & 0.00 & 0.87 & -15.2 & 0.988 \\
\hline $7: 129912783$ & rs111374282 & Intron & & 2.06 & 0.68 & 0.7 & $2.24 \times 10^{-1}$ & 0.00 & 0.87 & -15.2 & 0.988 \\
\hline $7: 129912602$ & rs2242337 & Intron & & 28.87 & 26.67 & 0.2 & $2.28 \times 10^{-1}$ & 13.83 & 23.48 & -0.5 & 0.147 \\
\hline $7: 129921282$ & rs79737155 & Intron & & 2.06 & 0.69 & 0.6 & $2.32 \times 10^{-1}$ & 0.00 & 0.87 & -15.2 & 0.988 \\
\hline $7: 129916792$ & rs772521 & Intron & & 2.06 & 0.70 & 0.6 & $2.37 \times 10^{-1}$ & 0.00 & 0.87 & -15.2 & 0.988 \\
\hline $7: 129913193$ & & Intron & & 0.52 & 0.33 & 1.1 & $2.68 \times 10^{-1}$ & 0.00 & 0.00 & NA & NA \\
\hline $7: 129916515$ & rs 1759 & Exon & Syn & 67.01 & 62.79 & 0.2 & $2.87 \times 10^{-1}$ & 67.02 & 66.09 & 0 & 0.944 \\
\hline $7: 129916738$ & rs11537129 & Intron & & 0.52 & 0.13 & 1.1 & $2.91 \times 10^{-1}$ & 0.00 & 0.00 & NA & NA \\
\hline $7: 129918302$ & rs139699694 & Intron & & 0.52 & 0.13 & 1.1 & $2.91 \times 10^{-1}$ & 0.00 & 0.00 & NA & NA \\
\hline $7: 129913367$ & rs18269827 & Intron & & 0.52 & 0.12 & 1.0 & $3.34 \times 10^{-1}$ & 0.00 & 0.00 & NA & NA \\
\hline $7: 129910463$ & rs2291457 & Intron & & 32.47 & 33.99 & -0.2 & $3.36 \times 10^{-1}$ & 51.06 & 40.79 & 0.4 & 0.179 \\
\hline $7: 129913535$ & rs376231 & Intron & & 35.71 & 38.36 & -0.5 & $3.94 \times 10^{-1}$ & 44.44 & 47.06 & -1.1 & 0.522 \\
\hline $7: 129913113$ & rs14822868 & Intron & & 0.52 & 0.18 & 0.8 & $4.32 \times 10^{-1}$ & 0.00 & 0.43 & -14 & 0.987 \\
\hline 7:129916709 & rs13891471 & Intron & & 0.52 & 0.18 & 0.8 & $4.48 \times 10^{-1}$ & 0.00 & 0.00 & NA & NA \\
\hline $7: 129918022$ & rs142361564 & Intron & & 0.52 & 0.18 & 0.8 & $4.48 \times 10^{-1}$ & 0.00 & 0.00 & NA & NA \\
\hline
\end{tabular}


Table C-4. (Continued)

\begin{tabular}{|c|c|c|c|c|c|c|c|c|c|c|c|}
\hline \multirow[b]{2}{*}{ Chr:Loc ${ }^{a}$} & \multirow[b]{2}{*}{ RSID } & \multirow[b]{2}{*}{$\begin{array}{c}\text { Loca- } \\
\text { tion }\end{array}$} & \multirow[b]{2}{*}{ Function } & \multicolumn{4}{|c|}{ Cohort $(n=4217)$} & \multicolumn{4}{|c|}{ Case-control $(n=162)$} \\
\hline & & & & $\begin{array}{c}\text { MAF\% in } \\
\text { patients } \\
\text { with AP } \\
(n=97)\end{array}$ & $\begin{array}{l}\text { MAF\% in } \\
\text { patients } \\
\text { without } \\
\text { AP } \\
(n=3220) \\
\end{array}$ & $\begin{array}{l}\text { Coeff- } \\
\text { icient }^{b}\end{array}$ & $P^{\mathbf{b}}$ & $\begin{array}{l}\text { MAF\% } \\
\text { in cases } \\
(n=47)\end{array}$ & $\begin{array}{c}\text { MAF\% in } \\
\text { controls } \\
(n=115)\end{array}$ & $\begin{array}{l}\text { Coeff- } \\
\text { icient }^{c}\end{array}$ & $P^{\mathbf{c}}$ \\
\hline $7: 129917130$ & rs729332 & Intron & & 64.43 & 61.89 & 0.1 & $4.51 \times 10^{-1}$ & 67.02 & 65.22 & 0.1 & 0.773 \\
\hline $7: 129921236$ & rs138455311 & Intron & & 0.52 & 0.61 & -0.6 & $5.70 \times 10^{-1}$ & 0.00 & 0.00 & NA & NA \\
\hline $7: 129915384$ & rs14829691 & Intron & & 0.52 & 0.20 & 0.6 & $5.78 \times 10^{-1}$ & 0.00 & 0.00 & NA & NA \\
\hline $7: 129913297$ & rs 1241681 & Intron & & 6.19 & 6.50 & -0.2 & $5.93 \times 10^{-1}$ & 3.19 & 4.78 & -0.4 & 0.578 \\
\hline $7: 129912437$ & rs7778449 & Intron & & 6.19 & 6.49 & -0.2 & $5.97 \times 10^{-1}$ & 3.19 & 4.78 & -0.4 & 0.578 \\
\hline $7: 129913519$ & & Intron & & 3.25 & 2.19 & 0.2 & $6.50 \times 10^{-1}$ & 3.95 & 2.13 & 0.5 & 0.573 \\
\hline $7: 129917763$ & rs6943732 & Intron & & 0.52 & 0.28 & 0.4 & $7.05 \times 10^{-1}$ & 0.00 & 0.00 & NA & NA \\
\hline $7: 129916390$ & rs5973876 & Intron & & 24.74 & 21.64 & -0.1 & $7.06 \times 10^{-1}$ & 28.72 & 23.04 & 0.1 & 0.76 \\
\hline $7: 129916030$ & rs9641869 & Intron & & 24.74 & 21.64 & -0.1 & $7.08 \times 10^{-1}$ & 28.72 & 23.04 & 0.1 & 0.76 \\
\hline $7: 129913187$ & & Intron & & 0.52 & 0.63 & 0.3 & $7.62 \times 10^{-1}$ & 0.00 & 0.00 & NA & NA \\
\hline $7: 129913521$ & & Intron & & 5.81 & 5.83 & -0.1 & $7.67 \times 10^{-1}$ & 11.63 & 4.46 & 1.3 & 0.014 \\
\hline $7: 129918947$ & rs 1244258 & Intron & & 0.52 & 0.22 & 0.3 & $7.78 \times 10^{-1}$ & 0.00 & 0.00 & NA & NA \\
\hline $7: 129929090$ & rs38772 & Intron & & 19.07 & 20.63 & 0.0 & $8.06 \times 10^{-1}$ & 24.47 & 25.22 & 0 & 0.97 \\
\hline $7: 129921366$ & rs73146777 & Intron & & 0.52 & 0.55 & 0.2 & $8.12 \times 10^{-1}$ & 0.00 & 0.87 & -14.4 & 0.989 \\
\hline $7: 129915931$ & rs12612 & Intron & & 2.06 & 1.19 & 0.1 & $8.38 \times 10^{-1}$ & 0.00 & 0.00 & NA & NA \\
\hline $7: 129929715$ & rs28537519 & $\begin{array}{l}\text { Down- } \\
\text { stream }\end{array}$ & & 18.23 & 19.88 & 0.0 & $8.68 \times 10^{-1}$ & 24.47 & 25.22 & 0 & 0.97 \\
\hline $7: 129917678$ & rs 144237288 & Exon & $\begin{array}{c}\text { Nonsyn } \\
\text { damaging }\end{array}$ & 0.52 & 0.18 & 0.2 & $8.77 \times 10^{-1}$ & 1.06 & 0.00 & 14.8 & 0.987 \\
\hline $7: 129917142$ & rs38734 & Intron & & 7.22 & 6.52 & 0.0 & $9.05 \times 10^{-1}$ & 3.19 & 5.22 & -0.5 & 0.497 \\
\hline $7: 129909554$ & rs6199765 & Exon & Nonsyn & 1.03 & 0.29 & 0.1 & $9.08 \times 10^{-1}$ & 0.00 & 0.43 & -14 & 0.987 \\
\hline $7: 129913569$ & rs112953454 & Intron & & 1.03 & 0.29 & 0.1 & $9.08 \times 10^{-1}$ & 0.00 & 0.43 & -14 & 0.987 \\
\hline $7: 129913522$ & rs191671331 & Intron & & 0.52 & 0.44 & 0.1 & $9.44 \times 10^{-1}$ & 0.00 & 0.00 & NA & NA \\
\hline $7: 129921360$ & rs1436277 & Intron & & 0.52 & 0.24 & 0.1 & $9.52 \times 10^{-1}$ & 0.00 & 0.00 & NA & NA \\
\hline $7: 129913207$ & rs7988728 & Intron & & 1.03 & 0.61 & 0.0 & $9.84 \times 10^{-1}$ & 0.00 & 0.00 & NA & NA \\
\hline
\end{tabular}


Table C-4. (Continued)

\begin{tabular}{|c|c|c|c|c|c|c|c|c|c|c|c|}
\hline \multirow[b]{2}{*}{ Chr:Loc } & \multirow[b]{2}{*}{ RSID } & \multirow[b]{2}{*}{$\begin{array}{c}\text { Loca- } \\
\text { tion }\end{array}$} & \multirow[b]{2}{*}{ Function } & \multicolumn{4}{|c|}{ Cohort $(n=4217)$} & \multicolumn{4}{|c|}{ Case-control $(n=162)$} \\
\hline & & & & $\begin{array}{c}\text { MAF\% in } \\
\text { patients } \\
\text { with AP } \\
(n=97)\end{array}$ & $\begin{array}{c}\text { MAF\% in } \\
\text { patients } \\
\text { without } \\
\text { AP } \\
(n=3220)\end{array}$ & $\begin{array}{l}\text { Coeff- } \\
\text { icient }^{b}\end{array}$ & $P^{\mathbf{b}}$ & $\begin{array}{l}\text { MAF\% } \\
\text { in cases } \\
(n=47)\end{array}$ & $\begin{array}{c}\text { MAF\% in } \\
\text { controls } \\
(n=115)\end{array}$ & $\begin{array}{l}\text { Coeff- } \\
\text { icient }^{c}\end{array}$ & $P^{\mathbf{c}}$ \\
\hline $7: 129928960$ & rs14872586 & Intron & & 0.00 & 0.12 & -13.1 & $9.93 \times 10^{-1}$ & 0.00 & 0.00 & $\mathrm{NA}$ & NA \\
\hline 7:129915959 & rs116884893 & Intron & & 0.00 & 0.64 & -14.8 & $9.93 \times 10^{-1}$ & 1.06 & 0.00 & 15.5 & 0.986 \\
\hline $7: 129918160$ & & Intron & & 0.00 & 0.04 & -12.8 & $9.93 \times 10^{-1}$ & 0.00 & 0.00 & NA & NA \\
\hline $7: 129929298$ & rs143283211 & Intron & & 0.00 & 0.23 & -14.7 & $9.93 \times 10^{-1}$ & 0.00 & 0.43 & -15.3 & 0.986 \\
\hline $7: 129913534$ & & Intron & & 0.00 & 0.66 & -15.0 & $9.93 \times 10^{-1}$ & 0.00 & 0.47 & -14.1 & 0.987 \\
\hline $7: 129919517$ & rs2291458 & Intron & & 0.00 & 0.11 & -13.3 & $9.94 \times 10^{-1}$ & 0.00 & 0.00 & NA & NA \\
\hline 7:129929649 & rs6953189 & $\begin{array}{l}\text { Down- } \\
\text { stream }\end{array}$ & & 0.00 & 0.10 & -13.1 & $9.94 \times 10^{-1}$ & 0.00 & 0.00 & NA & NA \\
\hline 7:129917612 & & Intron & & 0.00 & 0.02 & -13.2 & $9.94 \times 10^{-1}$ & 0.00 & 0.00 & NA & NA \\
\hline $7: 129913337$ & & Intron & & 0.00 & 0.05 & -11.7 & $9.94 \times 10^{-1}$ & 0.00 & 0.00 & NA & NA \\
\hline $7: 129913125$ & & Intron & & 0.52 & 0.09 & -13.6 & $9.94 \times 10^{-1}$ & 0.00 & 0.00 & NA & NA \\
\hline $7: 129918369$ & rs187321656 & Intron & & 0.00 & 0.47 & -14.9 & $9.94 \times 10^{-1}$ & 0.00 & 0.43 & -13.3 & 0.988 \\
\hline 7:129913181 & & Intron & & 0.00 & 0.45 & -14.6 & $9.94 \times 10^{-1}$ & 0.00 & 0.00 & NA & NA \\
\hline $7: 129917256$ & & Intron & & 0.00 & 0.01 & -11.4 & $9.94 \times 10^{-1}$ & 0.00 & 0.00 & NA & NA \\
\hline 7:129918306 & & Intron & & 0.00 & 0.04 & -13 & $9.94 \times 10^{-1}$ & 0.00 & 0.00 & NA & NA \\
\hline $7: 129912250$ & & Intron & & 0.00 & 0.04 & -12.8 & $9.94 \times 10^{-1}$ & 0.00 & 0.00 & NA & NA \\
\hline $7: 129916927$ & rs181854161 & Intron & & 0.00 & 0.18 & -14.0 & $9.94 \times 10^{-1}$ & 0.00 & 0.43 & -13.3 & 0.988 \\
\hline $7: 129913498$ & & Intron & & 0.00 & 0.04 & -13.0 & $9.94 \times 10^{-1}$ & 0.00 & 0.00 & NA & NA \\
\hline 7:129912859 & & Intron & & 0.00 & 0.01 & -11.7 & $9.94 \times 10^{-1}$ & 0.00 & 0.00 & NA & NA \\
\hline 7:129916607 & rs373726453 & Intron & & 0.00 & 0.04 & -12.7 & $9.94 \times 10^{-1}$ & 0.00 & 0.00 & NA & NA \\
\hline $7: 129913512$ & & Intron & & 0.00 & 0.02 & -12.2 & $9.94 \times 10^{-1}$ & 0.00 & 0.00 & NA & NA \\
\hline 7:129916836 & rs73476856 & Intron & & 0.52 & 0.18 & -14.7 & $9.94 \times 10^{-1}$ & 0.00 & 0.00 & NA & NA \\
\hline $7: 129913894$ & & Intron & & 0.00 & 0.01 & -10.9 & $9.94 \times 10^{-1}$ & 0.00 & 0.00 & NA & NA \\
\hline 7:129917161 & & Intron & & 0.00 & 0.01 & -10.9 & $9.94 \times 10^{-1}$ & 0.00 & 0.00 & NA & NA \\
\hline $7: 129909598$ & rs 146466435 & Exon & Syn & 0.00 & 0.07 & -12.8 & $9.94 \times 10^{-1}$ & 0.00 & 0.00 & NA & NA \\
\hline
\end{tabular}


Table C-4. (Continued)

\begin{tabular}{|c|c|c|c|c|c|c|c|c|c|c|}
\hline \multirow[b]{2}{*}{ Chr:Loc ${ }^{a}$} & \multirow[b]{2}{*}{ RSID } & \multirow[b]{2}{*}{$\begin{array}{l}\text { Loca- Function } \\
\text { tion }\end{array}$} & \multicolumn{4}{|c|}{ Cohort $(n=4217)$} & \multicolumn{4}{|c|}{ Case-control $(n=162)$} \\
\hline & & & $\begin{array}{c}\text { MAF\% in } \\
\text { patients } \\
\text { with AP } \\
(n=97)\end{array}$ & $\begin{array}{c}\text { MAF\% in } \\
\text { patients } \\
\text { without } \\
\text { AP } \\
(n=3220) \\
\end{array}$ & $\begin{array}{l}\text { Coeff- } \\
\text { icient }^{b}\end{array}$ & $P^{\mathbf{b}}$ & $\begin{array}{l}\text { MAF\% } \\
\text { in cases } \\
(n=47)\end{array}$ & $\begin{array}{c}\text { MAF\% in } \\
\text { controls } \\
(n=115)\end{array}$ & $\begin{array}{l}\text { Coeff- } \\
\text { icient }^{c}\end{array}$ & $P^{\mathbf{c}}$ \\
\hline $7: 129909672$ & & Intron & 0.00 & 0.02 & -12.2 & $9.94 \times 10^{-1}$ & 0.00 & 0.00 & $\mathrm{NA}$ & NA \\
\hline $7: 129921924$ & rs11583336 & Intron & 0.00 & 0.41 & -15.7 & $9.94 \times 10^{-1}$ & 0.00 & 0.00 & NA & NA \\
\hline $7: 129912748$ & & Intron & 0.00 & 0.04 & -12.4 & $9.94 \times 10^{-1}$ & 0.00 & 0.00 & NA & NA \\
\hline 7:129917991 & & Intron & 0.00 & 0.02 & -12.6 & $9.94 \times 10^{-1}$ & 0.00 & 0.00 & NA & NA \\
\hline $7: 129916068$ & rs 116555283 & Intron & 0.00 & 0.40 & -15.7 & $9.94 \times 10^{-1}$ & 0.00 & 0.00 & NA & NA \\
\hline $7: 129918337$ & & Intron & 0.00 & 0.02 & -12.7 & $9.94 \times 10^{-1}$ & 0.00 & 0.00 & NA & NA \\
\hline $7: 129928692$ & & Intron & 0.00 & 0.01 & -13.2 & $9.94 \times 10^{-1}$ & 0.00 & 0.00 & NA & NA \\
\hline $7: 129914855$ & & Intron & 0.00 & 0.09 & -13.2 & $9.94 \times 10^{-1}$ & 0.00 & 0.00 & NA & NA \\
\hline 7:129910456 & rs11474756 & Intron & 0.00 & 0.39 & -15.7 & $9.95 \times 10^{-1}$ & 0.00 & 0.00 & NA & NA \\
\hline 7:129916445 & rs 1523838 & Intron & 0.00 & 0.39 & -15.7 & $9.95 \times 10^{-1}$ & 0.00 & 0.00 & NA & NA \\
\hline 7:129917844 & rs18987654 & Intron & 0.00 & 0.21 & -13.8 & $9.95 \times 10^{-1}$ & 1.09 & 0.43 & 1.3 & 0.353 \\
\hline $7: 129909727$ & & Intron & 0.00 & 0.11 & -12.7 & $9.95 \times 10^{-1}$ & 0.00 & 0.00 & NA & NA \\
\hline 7:129913397 & & Intron & 0.00 & 0.35 & -15.0 & $9.95 \times 10^{-1}$ & 0.00 & 0.00 & NA & NA \\
\hline $7: 129913635$ & rs115348366 & Intron & 0.00 & 0.38 & -15.7 & $9.95 \times 10^{-1}$ & 0.00 & 0.00 & NA & NA \\
\hline 7:129906649 & & $\begin{array}{l}\text { Up- } \\
\text { stream }\end{array}$ & 0.00 & 0.01 & -10.8 & $9.95 \times 10^{-1}$ & 0.00 & 0.00 & NA & NA \\
\hline $7: 129916273$ & & Intron & 0.00 & 0.01 & -12.7 & $9.95 \times 10^{-1}$ & 0.00 & 0.00 & NA & NA \\
\hline 7:129913206 & & Intron & 0.00 & 0.04 & -12 & $9.95 \times 10^{-1}$ & 0.00 & 0.00 & NA & NA \\
\hline $7: 129928650$ & & Intron & 0.00 & 0.02 & -10.9 & $9.95 \times 10^{-1}$ & 0.00 & 0.00 & NA & NA \\
\hline $7: 129918673$ & & Intron & 0.00 & 0.01 & -12.7 & $9.95 \times 10^{-1}$ & 0.00 & 0.00 & NA & NA \\
\hline 7:129929366 & rs3737297 & Intron & 0.00 & 0.02 & -10.9 & $9.95 \times 10^{-1}$ & 0.00 & 0.00 & NA & NA \\
\hline $7: 129915582$ & & Intron & 0.00 & 0.01 & -12.7 & $9.95 \times 10^{-1}$ & 0.00 & 0.00 & NA & NA \\
\hline $7: 129917273$ & & Intron & 0.00 & 0.01 & -10.6 & $9.95 \times 10^{-1}$ & 0.00 & 0.00 & NA & NA \\
\hline $7: 129914105$ & & Intron & 0.00 & 0.02 & -11.8 & $9.95 \times 10^{-1}$ & 0.00 & 0.00 & NA & NA \\
\hline $7: 129921337$ & & Intron & 0.00 & 0.01 & -10.7 & $9.95 \times 10^{-1}$ & 0.00 & 0.43 & -13.3 & 0.988 \\
\hline
\end{tabular}


Table C-4. (Continued)

\begin{tabular}{|c|c|c|c|c|c|c|c|c|c|c|c|}
\hline \multirow[b]{2}{*}{ Chr:Loc ${ }^{a}$} & \multirow[b]{2}{*}{ RSID } & \multirow[b]{2}{*}{$\begin{array}{c}\text { Loca- } \\
\text { tion }\end{array}$} & \multirow[b]{2}{*}{ Function } & \multicolumn{4}{|c|}{ Cohort $(n=4217)$} & \multicolumn{4}{|c|}{ Case-control $(n=162)$} \\
\hline & & & & $\begin{array}{c}\text { MAF\% in } \\
\text { patients } \\
\text { with AP } \\
(n=97)\end{array}$ & $\begin{array}{c}\text { MAF\% in } \\
\text { patients } \\
\text { without } \\
\text { AP } \\
(n=3220)\end{array}$ & $\begin{array}{l}\text { Coeff- } \\
\text { icient }^{b}\end{array}$ & $P^{\mathbf{b}}$ & $\begin{array}{l}\text { MAF\% } \\
\text { in cases } \\
(n=47)\end{array}$ & $\begin{array}{c}\text { MAF\% in } \\
\text { controls } \\
(n=115)\end{array}$ & $\begin{array}{l}\text { Coeff- } \\
\text { icient }^{c}\end{array}$ & $P^{\mathfrak{c}}$ \\
\hline $7: 129928834$ & & Intron & & 0.00 & 0.01 & -13.1 & $9.95 \times 10^{-1}$ & 0.00 & 0.00 & $\mathrm{NA}$ & $\mathrm{NA}$ \\
\hline 7:129914996 & & Exon & $\begin{array}{l}\text { Nonsyn } \\
\text { damaging }\end{array}$ & 0.00 & 0.02 & -10.2 & $9.95 \times 10^{-1}$ & 0.00 & 0.00 & NA & NA \\
\hline $7: 129929488$ & rs 1415849 & Exon & Syn & 0.00 & 0.04 & -11.3 & $9.95 \times 10^{-1}$ & 0.00 & 0.00 & NA & NA \\
\hline 7:129929694 & & $\begin{array}{l}\text { Down- } \\
\text { stream }\end{array}$ & & 0.00 & 0.01 & -10.7 & $9.95 \times 10^{-1}$ & 0.00 & 0.00 & NA & NA \\
\hline $7: 129916375$ & & Intron & & 0.00 & 0.04 & -11.9 & $9.95 \times 10^{-1}$ & 0.00 & 0.00 & NA & NA \\
\hline $7: 129913426$ & & Intron & & 0.00 & 0.02 & -11.9 & $9.95 \times 10^{-1}$ & 0.00 & 0.00 & NA & NA \\
\hline 7:129918886 & & Intron & & 0.00 & 0.04 & -11.2 & $9.95 \times 10^{-1}$ & 0.00 & 0.00 & NA & NA \\
\hline $7: 129906657$ & & $\begin{array}{c}\text { Up- } \\
\text { stream }\end{array}$ & & 0.00 & 0.01 & -12.4 & $9.95 \times 10^{-1}$ & 0.00 & 0.43 & -13.5 & 0.988 \\
\hline 7:129913339 & & Intron & & 0.00 & 0.02 & -12.4 & $9.95 \times 10^{-1}$ & 0.00 & 0.00 & NA & NA \\
\hline $7: 129915314$ & & Intron & & 0.00 & 0.01 & -12.5 & $9.95 \times 10^{-1}$ & 0.00 & 0.43 & -13.4 & 0.988 \\
\hline 7:129919091 & & Intron & & 0.00 & 0.01 & -12.4 & $9.95 \times 10^{-1}$ & 0.00 & 0.00 & NA & NA \\
\hline $7: 129929682$ & rs373156748 & $\begin{array}{l}\text { Down- } \\
\text { stream }\end{array}$ & & 0.00 & 0.02 & -12.6 & $9.95 \times 10^{-1}$ & 0.00 & 0.43 & -13.8 & 0.988 \\
\hline 7:129908963 & & Intron & & 0.00 & 0.01 & -12.4 & $9.95 \times 10^{-1}$ & 0.00 & 0.00 & NA & NA \\
\hline 7:129919373 & rs151137985 & Exon & Syn & 0.00 & 0.15 & -14.1 & $9.95 \times 10^{-1}$ & 0.00 & 0.43 & -13.3 & 0.988 \\
\hline $7: 129915643$ & & Intron & & 0.00 & 0.01 & -13.1 & $9.95 \times 10^{-1}$ & 0.00 & 0.00 & NA & NA \\
\hline 7:129917292 & & Intron & & 0.00 & 0.04 & -13.7 & $9.95 \times 10^{-1}$ & 0.00 & 0.00 & NA & NA \\
\hline 7:129917909 & & Intron & & 0.00 & 0.01 & -10.1 & $9.95 \times 10^{-1}$ & 0.00 & 0.00 & NA & NA \\
\hline $7: 129916653$ & & Intron & & 0.00 & 0.01 & -12.2 & $9.95 \times 10^{-1}$ & 0.00 & 0.00 & NA & NA \\
\hline 7:129921096 & & Intron & & 0.00 & 0.02 & -11.8 & $9.95 \times 10^{-1}$ & 0.00 & 0.00 & NA & NA \\
\hline $7: 129921822$ & & Intron & & 0.00 & 0.02 & -12.0 & $9.95 \times 10^{-1}$ & 0.00 & 0.00 & NA & NA \\
\hline 7:129919472 & rs6199762 & Exon & Syn & 0.00 & 0.01 & -12.1 & $9.95 \times 10^{-1}$ & 0.00 & 0.00 & NA & NA \\
\hline $7: 129912195$ & & Intron & & 0.00 & 0.01 & -12.6 & $9.95 \times 10^{-1}$ & 0.00 & 0.00 & NA & NA \\
\hline
\end{tabular}


Table C-4. (Continued)

\begin{tabular}{|c|c|c|c|c|c|c|c|c|c|c|c|}
\hline \multirow[b]{2}{*}{ Chr:Loc ${ }^{a}$} & \multirow[b]{2}{*}{ RSID } & \multirow[b]{2}{*}{$\begin{array}{c}\text { Loca- } \\
\text { tion }\end{array}$} & \multirow[b]{2}{*}{ Function } & \multicolumn{4}{|c|}{ Cohort $(n=4217)$} & \multicolumn{4}{|c|}{ Case-control $(n=162)$} \\
\hline & & & & $\begin{array}{c}\text { MAF\% in } \\
\text { patients } \\
\text { with AP } \\
(n=97)\end{array}$ & $\begin{array}{l}\text { MAF\% in } \\
\text { patients } \\
\text { without } \\
\text { AP } \\
(n=3220)\end{array}$ & $\begin{array}{l}\text { Coeff- } \\
\text { icient }^{b}\end{array}$ & $P^{\mathbf{b}}$ & $\begin{array}{l}\text { MAF\% } \\
\text { in cases } \\
(n=47)\end{array}$ & $\begin{array}{c}\mathrm{MAF} \% \text { in } \\
\text { controls } \\
(n=115)\end{array}$ & $\begin{array}{l}\text { Coeff- } \\
\text { icient }^{c}\end{array}$ & $P^{\mathbf{c}}$ \\
\hline $7: 129915203$ & & Intron & & 0.00 & 0.07 & -13.3 & $9.95 \times 10^{-1}$ & 0.00 & 0.00 & NA & $\mathrm{NA}$ \\
\hline $7: 129917640$ & rs36936412 & Intron & & 0.00 & 0.01 & -12.5 & $9.95 \times 10^{-1}$ & 0.00 & 0.00 & NA & NA \\
\hline $7: 129913227$ & & Intron & & 0.00 & 0.01 & -12 & $9.95 \times 10^{-1}$ & 0.00 & 0.00 & NA & NA \\
\hline 7:129909574 & rs37757816 & Exon & Syn & 0.00 & 0.01 & -12.5 & $9.95 \times 10^{-1}$ & 0.00 & 0.00 & NA & NA \\
\hline 7:129918877 & & Intron & & 0.00 & 0.08 & -13.4 & $9.95 \times 10^{-1}$ & 0.00 & 0.00 & NA & NA \\
\hline $7: 129913064$ & & Intron & & 0.00 & 0.01 & -10.6 & $9.95 \times 10^{-1}$ & 0.00 & 0.00 & NA & NA \\
\hline $7: 129919025$ & & Intron & & 0.00 & 0.01 & -10.6 & $9.95 \times 10^{-1}$ & 0.00 & 0.00 & NA & NA \\
\hline $7: 129915432$ & & Intron & & 0.00 & 0.02 & -12.0 & $9.95 \times 10^{-1}$ & 0.00 & 0.00 & NA & NA \\
\hline 7:129922036 & & Intron & & 0.00 & 0.01 & -11.9 & $9.95 \times 10^{-1}$ & 0.00 & 0.00 & NA & NA \\
\hline $7: 129922130$ & & Intron & & 0.00 & 0.01 & -11.9 & $9.95 \times 10^{-1}$ & 0.00 & 0.00 & NA & NA \\
\hline 7:129916138 & & Intron & & 0.00 & 0.01 & -11.9 & $9.95 \times 10^{-1}$ & 0.00 & 0.00 & NA & NA \\
\hline $7: 129928818$ & & Intron & & 0.00 & 0.01 & -11.9 & $9.95 \times 10^{-1}$ & 0.00 & 0.00 & NA & NA \\
\hline 7:129913210 & & Intron & & 0.00 & 0.01 & -11.9 & $9.95 \times 10^{-1}$ & 0.00 & 0.00 & NA & NA \\
\hline $7: 129929484$ & rs 14662328 & Exon & Nonsyn & 0.00 & 0.07 & -12.8 & $9.95 \times 10^{-1}$ & 0.00 & 0.00 & NA & NA \\
\hline 7:129917796 & rs 14873587 & Intron & & 0.00 & 0.13 & -14.5 & $9.95 \times 10^{-1}$ & 0.00 & 0.00 & NA & NA \\
\hline $7: 129917035$ & & Intron & & 0.00 & 0.01 & -12.2 & $9.95 \times 10^{-1}$ & 0.00 & 0.00 & NA & NA \\
\hline $7: 129915677$ & & Intron & & 0.00 & 0.01 & -11.9 & $9.95 \times 10^{-1}$ & 0.00 & 0.00 & NA & NA \\
\hline $7: 129928707$ & & Intron & & 0.00 & 0.01 & -11.9 & $9.95 \times 10^{-1}$ & 0.00 & 0.00 & NA & NA \\
\hline 7:129921120 & & Intron & & 0.00 & 0.01 & -11.9 & $9.95 \times 10^{-1}$ & 0.00 & 0.00 & NA & NA \\
\hline 7:129914143 & & Intron & & 0.00 & 0.01 & -11.9 & $9.95 \times 10^{-1}$ & 0.00 & 0.00 & NA & NA \\
\hline $7: 129913542$ & & Intron & & 0.00 & 0.05 & -12.7 & $9.95 \times 10^{-1}$ & 0.00 & 0.00 & NA & NA \\
\hline 7:129928912 & & Intron & & 0.00 & 0.01 & -12.3 & $9.95 \times 10^{-1}$ & 0.00 & 0.00 & NA & NA \\
\hline $7: 129918717$ & & Intron & & 0.00 & 0.01 & -11.9 & $9.95 \times 10^{-1}$ & 0.00 & 0.00 & NA & NA \\
\hline $7: 129917225$ & & Intron & & 0.00 & 0.01 & -11.9 & $9.95 \times 10^{-1}$ & 0.00 & 0.00 & NA & NA \\
\hline $7: 129915294$ & & Intron & & 0.00 & 0.01 & -12.4 & $9.95 \times 10^{-1}$ & 0.00 & 0.00 & NA & NA \\
\hline
\end{tabular}


Table C-4. (Continued)

\begin{tabular}{|c|c|c|c|c|c|c|c|c|c|c|c|}
\hline \multirow[b]{2}{*}{ Chr:Loc ${ }^{a}$} & \multirow[b]{2}{*}{ RSID } & \multirow[b]{2}{*}{$\begin{array}{c}\text { Loca- } \\
\text { tion }\end{array}$} & \multirow[b]{2}{*}{ Function } & \multicolumn{4}{|c|}{ Cohort $(n=4217)$} & \multicolumn{4}{|c|}{ Case-control $(n=162)$} \\
\hline & & & & $\begin{array}{c}\text { MAF\% in } \\
\text { patients } \\
\text { with AP } \\
(n=97)\end{array}$ & $\begin{array}{l}\text { MAF\% in } \\
\text { patients } \\
\text { without } \\
\text { AP } \\
(n=3220)\end{array}$ & $\begin{array}{l}\text { Coeff- } \\
\text { icient }^{b}\end{array}$ & $P^{\mathbf{b}}$ & $\begin{array}{l}\text { MAF\% } \\
\text { in cases } \\
(n=47)\end{array}$ & $\begin{array}{c}\text { MAF\% in } \\
\text { controls } \\
(n=115)\end{array}$ & $\begin{array}{l}\text { Coeff- } \\
\text { icient }^{c}\end{array}$ & $P^{\mathbf{c}}$ \\
\hline $7: 129915471$ & & Intron & & 0.00 & 0.01 & -11.9 & $9.95 \times 10^{-1}$ & 0.00 & 0.00 & NA & NA \\
\hline $7: 129906630$ & & $\begin{array}{c}\text { Up- } \\
\text { stream }\end{array}$ & & 0.00 & 0.01 & -12.2 & $9.95 \times 10^{-1}$ & 0.00 & 0.00 & NA & NA \\
\hline 7:129929706 & & $\begin{array}{l}\text { Down- } \\
\text { stream }\end{array}$ & & 0.00 & 0.01 & -12.4 & $9.95 \times 10^{-1}$ & 0.00 & 0.00 & NA & NA \\
\hline 7:129929716 & & $\begin{array}{l}\text { Down- } \\
\text { stream }\end{array}$ & & 0.00 & 0.01 & -11.8 & $9.95 \times 10^{-1}$ & 0.00 & 0.00 & NA & NA \\
\hline $7: 129913920$ & & Intron & & 0.00 & 0.02 & -12.9 & $9.95 \times 10^{-1}$ & 0.00 & 0.00 & NA & NA \\
\hline 7:129916729 & & Intron & & 0.00 & 0.01 & -11.8 & $9.95 \times 10^{-1}$ & 0.00 & 0.00 & NA & NA \\
\hline 7:129919020 & & Intron & & 0.00 & 0.01 & -11.8 & $9.95 \times 10^{-1}$ & 0.00 & 0.00 & NA & NA \\
\hline $7: 129912816$ & & Intron & & 0.00 & 0.01 & -11.7 & $9.95 \times 10^{-1}$ & 0.00 & 0.00 & NA & NA \\
\hline 7:129909434 & & Intron & & 0.00 & 0.01 & -11.7 & $9.95 \times 10^{-1}$ & 0.00 & 0.00 & NA & NA \\
\hline $7: 129912305$ & & Intron & & 0.00 & 0.02 & -12.0 & $9.95 \times 10^{-1}$ & 0.00 & 0.00 & NA & NA \\
\hline $7: 129909575$ & rs 142582879 & Exon & Nonsyn & 0.00 & 0.01 & -11.6 & $9.95 \times 10^{-1}$ & 0.00 & 0.00 & NA & NA \\
\hline 7:129906791 & rs376131992 & Exon & $\begin{array}{c}\text { Nonsyn } \\
\text { damaging }\end{array}$ & 0.00 & 0.01 & -11.6 & $9.95 \times 10^{-1}$ & 0.00 & 0.00 & NA & NA \\
\hline 7:129908878 & & Intron & & 0.00 & 0.01 & -12.7 & $9.95 \times 10^{-1}$ & 0.00 & 0.00 & NA & NA \\
\hline 7:129912704 & & Intron & & 0.00 & 0.01 & -11.6 & $9.95 \times 10^{-1}$ & 0.00 & 0.00 & NA & NA \\
\hline 7:129918402 & & Intron & & 0.00 & 0.01 & -12.4 & $9.95 \times 10^{-1}$ & 0.00 & 0.00 & NA & NA \\
\hline 7:129918422 & & Intron & & 0.00 & 0.01 & -11.5 & $9.95 \times 10^{-1}$ & 0.00 & 0.00 & NA & NA \\
\hline $7: 129915570$ & & Intron & & 0.00 & 0.02 & -11.6 & $9.95 \times 10^{-1}$ & 0.00 & 0.00 & NA & NA \\
\hline $7: 129916824$ & & Intron & & 0.00 & 0.01 & -12.0 & $9.95 \times 10^{-1}$ & 0.00 & 0.00 & NA & NA \\
\hline $7: 129917027$ & & Intron & & 0.00 & 0.01 & -11.5 & $9.95 \times 10^{-1}$ & 0.00 & 0.00 & NA & NA \\
\hline 7:129919529 & & Intron & & 0.00 & 0.01 & -11.9 & $9.95 \times 10^{-1}$ & 0.00 & 0.00 & NA & NA \\
\hline $7: 129913523$ & & Intron & & 0.00 & 0.06 & -13 & $9.95 \times 10^{-1}$ & 0.00 & 0.00 & NA & NA \\
\hline $7: 129915355$ & & Intron & & 0.00 & 0.01 & -11.5 & $9.95 \times 10^{-1}$ & 0.00 & 0.00 & NA & NA \\
\hline
\end{tabular}


Table C-4. (Continued)

\begin{tabular}{|c|c|c|c|c|c|c|c|c|c|c|c|}
\hline \multirow[b]{2}{*}{ Chr:Loc ${ }^{a}$} & \multirow[b]{2}{*}{ RSID } & \multirow[b]{2}{*}{$\begin{array}{c}\text { Loca- } \\
\text { tion }\end{array}$} & \multirow[b]{2}{*}{ Function } & \multicolumn{4}{|c|}{ Cohort $(n=4217)$} & \multicolumn{4}{|c|}{ Case-control $(n=162)$} \\
\hline & & & & $\begin{array}{c}\text { MAF\% in } \\
\text { patients } \\
\text { with AP } \\
(n=97)\end{array}$ & $\begin{array}{c}\text { MAF\% in } \\
\text { patients } \\
\text { without } \\
\text { AP } \\
(n=3220)\end{array}$ & $\begin{array}{l}\text { Coeff- } \\
\text { icient }^{b}\end{array}$ & $P^{\mathbf{b}}$ & $\begin{array}{l}\text { MAF\% } \\
\text { in cases } \\
(n=47)\end{array}$ & $\begin{array}{c}\mathrm{MAF} \% \text { in } \\
\text { controls } \\
(\mathrm{n}=115)\end{array}$ & $\begin{array}{l}\text { Coeff- } \\
\text { icient }^{c}\end{array}$ & $P^{\mathbf{c}}$ \\
\hline $7: 129906595$ & & $\begin{array}{c}\text { Up- } \\
\text { stream }\end{array}$ & & 0.00 & 0.02 & -13.6 & $9.95 \times 10^{-1}$ & 0.00 & 0.00 & $\mathrm{NA}$ & NA \\
\hline $7: 129913127$ & & Intron & & 0.00 & 0.01 & -11.8 & $9.95 \times 10^{-1}$ & 0.00 & 0.00 & NA & NA \\
\hline $7: 129929035$ & & Intron & & 0.00 & 0.01 & -11.9 & $9.95 \times 10^{-1}$ & 0.00 & 0.00 & NA & NA \\
\hline $7: 129921169$ & & Intron & & 0.00 & 0.01 & -11.9 & $9.95 \times 10^{-1}$ & 0.00 & 0.00 & NA & NA \\
\hline 7:129917832 & & Intron & & 0.00 & 0.04 & -12.5 & $9.95 \times 10^{-1}$ & 1.06 & 0.00 & 15.9 & 0.986 \\
\hline $7: 129919343$ & rs17591 & Exon & Syn & 0.00 & 0.32 & -15.0 & $9.95 \times 10^{-1}$ & 1.06 & 0.00 & 15.9 & 0.986 \\
\hline $7: 129915955$ & & Intron & & 0.00 & 0.01 & -11.5 & $9.95 \times 10^{-1}$ & 0.00 & 0.00 & NA & NA \\
\hline 7:129917249 & & Intron & & 0.00 & 0.01 & -9.9 & $9.95 \times 10^{-1}$ & 0.00 & 0.00 & NA & NA \\
\hline $7: 129921087$ & rs183914994 & Intron & & 0.00 & 0.01 & -11.7 & $9.95 \times 10^{-1}$ & 0.00 & 0.00 & NA & NA \\
\hline 7:129917339 & rs18727464 & Intron & & 0.00 & 0.06 & -12.8 & $9.95 \times 10^{-1}$ & 0.00 & 0.00 & NA & NA \\
\hline 7:129917368 & & Intron & & 0.00 & 0.01 & -11.9 & $9.95 \times 10^{-1}$ & 0.00 & 0.00 & NA & NA \\
\hline $7: 129912413$ & & Intron & & 0.00 & 0.01 & -11.3 & $9.95 \times 10^{-1}$ & 0.00 & 0.00 & NA & NA \\
\hline $7: 129918387$ & & Intron & & 0.00 & 0.01 & -11.3 & $9.95 \times 10^{-1}$ & 0.00 & 0.00 & NA & NA \\
\hline $7: 129919244$ & & Intron & & 0.00 & 0.01 & -9.8 & $9.95 \times 10^{-1}$ & 0.00 & 0.00 & NA & NA \\
\hline $7: 129918064$ & & Intron & & 0.00 & 0.01 & -9.8 & $9.95 \times 10^{-1}$ & 0.00 & 0.00 & NA & NA \\
\hline $7: 129922154$ & & Intron & & 0.00 & 0.01 & -9.6 & $9.95 \times 10^{-1}$ & 0.00 & 0.00 & NA & NA \\
\hline $7: 129915511$ & rs 18355629 & Intron & & 0.00 & 0.13 & -13.9 & $9.95 \times 10^{-1}$ & 0.00 & 0.00 & NA & NA \\
\hline 7:129915411 & & Intron & & 0.00 & 0.01 & -11.5 & $9.95 \times 10^{-1}$ & 0.00 & 0.00 & NA & NA \\
\hline 7:129914008 & & Intron & & 0.00 & 0.01 & -11.2 & $9.95 \times 10^{-1}$ & 0.00 & 0.00 & NA & NA \\
\hline 7:129914011 & & Intron & & 0.00 & 0.01 & -11.2 & $9.95 \times 10^{-1}$ & 0.00 & 0.00 & NA & NA \\
\hline 7:129915197 & & Intron & & 0.00 & 0.01 & -11.5 & $9.95 \times 10^{-1}$ & 0.00 & 0.00 & NA & NA \\
\hline $7: 129916627$ & & Intron & & 0.00 & 0.01 & -11.4 & $9.95 \times 10^{-1}$ & 0.00 & 0.00 & NA & NA \\
\hline $7: 129915103$ & & Intron & & 0.00 & 0.02 & -12.7 & $9.95 \times 10^{-1}$ & 0.00 & 0.00 & NA & NA \\
\hline $7: 129929316$ & & Intron & & 0.00 & 0.01 & -11.1 & $9.95 \times 10^{-1}$ & 0.00 & 0.00 & NA & NA \\
\hline
\end{tabular}


Table C-4. (Continued)

\begin{tabular}{|c|c|c|c|c|c|c|c|c|c|c|c|}
\hline \multirow[b]{2}{*}{ Chr:Loc ${ }^{a}$} & \multirow[b]{2}{*}{ RSID } & \multirow[b]{2}{*}{$\begin{array}{c}\text { Loca- } \\
\text { tion }\end{array}$} & \multirow[b]{2}{*}{ Function } & \multicolumn{4}{|c|}{ Cohort $(n=4217)$} & \multicolumn{4}{|c|}{ Case-control $(n=162)$} \\
\hline & & & & $\begin{array}{c}\text { MAF\% in } \\
\text { patients } \\
\text { with AP } \\
(n=97)\end{array}$ & $\begin{array}{c}\text { MAF\% in } \\
\text { patients } \\
\text { without } \\
\text { AP } \\
(n=3220)\end{array}$ & $\begin{array}{l}\text { Coeff- } \\
\text { icient }^{b}\end{array}$ & $P^{\mathbf{b}}$ & $\begin{array}{l}\text { MAF\% } \\
\text { in cases } \\
(n=47)\end{array}$ & $\begin{array}{c}\mathrm{MAF} \% \text { in } \\
\text { controls } \\
(\mathrm{n}=115)\end{array}$ & $\begin{array}{l}\text { Coeff- } \\
\text { icient }^{c}\end{array}$ & $P^{\mathbf{c}}$ \\
\hline $7: 129919002$ & & Intron & & 0.00 & 0.06 & -13.0 & $9.95 \times 10^{-1}$ & 0.00 & 0.00 & NA & $\mathrm{NA}$ \\
\hline 7:129913784 & & Intron & & 0.00 & 0.01 & -11.8 & $9.95 \times 10^{-1}$ & 0.00 & 0.00 & NA & NA \\
\hline $7: 129929604$ & rs373858339 & UTR3 & & 0.00 & 0.01 & -11.8 & $9.95 \times 10^{-1}$ & 0.00 & 0.00 & NA & NA \\
\hline $7: 129912430$ & & Intron & & 0.00 & 0.15 & -14 & $9.95 \times 10^{-1}$ & 0.00 & 0.00 & NA & NA \\
\hline 7:129917141 & & Intron & & 0.00 & 0.01 & -11.0 & $9.95 \times 10^{-1}$ & 0.00 & 0.00 & NA & NA \\
\hline $7: 129918804$ & & Intron & & 0.00 & 0.01 & -11.0 & $9.95 \times 10^{-1}$ & 0.00 & 0.00 & NA & NA \\
\hline $7: 129912807$ & & Intron & & 0.00 & 0.01 & -11.0 & $9.95 \times 10^{-1}$ & 0.00 & 0.00 & NA & NA \\
\hline $7: 129916431$ & rs 199873336 & Intron & & 0.00 & 0.01 & -11.0 & $9.95 \times 10^{-1}$ & 0.00 & 0.00 & NA & NA \\
\hline $7: 129914923$ & & Intron & & 0.00 & 0.01 & -11.0 & $9.95 \times 10^{-1}$ & 0.00 & 0.00 & NA & NA \\
\hline $7: 129928939$ & & Intron & & 0.00 & 0.01 & -11.0 & $9.95 \times 10^{-1}$ & 0.00 & 0.00 & NA & NA \\
\hline 7:129913992 & rs 18736757 & Intron & & 0.00 & 0.01 & -11.5 & $9.95 \times 10^{-1}$ & 0.00 & 0.00 & NA & NA \\
\hline $7: 129917111$ & & Intron & & 0.00 & 0.01 & -11.0 & $9.95 \times 10^{-1}$ & 0.00 & 0.00 & NA & NA \\
\hline $7: 129917623$ & rs3762143 & Intron & & 0.00 & 0.04 & -11.9 & $9.95 \times 10^{-1}$ & 0.00 & 0.00 & NA & NA \\
\hline $7: 129913067$ & & Intron & & 0.00 & 0.01 & -10.9 & $9.95 \times 10^{-1}$ & 0.00 & 0.00 & NA & NA \\
\hline $7: 129929713$ & & $\begin{array}{l}\text { Down- } \\
\text { stream }\end{array}$ & & 0.00 & 0.01 & -11.9 & $9.95 \times 10^{-1}$ & 0.00 & 0.00 & NA & NA \\
\hline $7: 129919169$ & & Intron & & 0.00 & 0.01 & -11.6 & $9.95 \times 10^{-1}$ & 0.00 & 0.00 & NA & NA \\
\hline $7: 129917811$ & & Intron & & 0.00 & 0.02 & -13 & $9.95 \times 10^{-1}$ & 0.00 & 0.00 & NA & NA \\
\hline 7:129913994 & & Intron & & 0.00 & 0.04 & -11.5 & $9.95 \times 10^{-1}$ & 0.00 & 0.00 & NA & NA \\
\hline $7: 129918027$ & & Intron & & 0.00 & 0.01 & -11.3 & $9.95 \times 10^{-1}$ & 0.00 & 0.00 & NA & NA \\
\hline $7: 129915200$ & & Intron & & 0.00 & 0.01 & -10.9 & $9.95 \times 10^{-1}$ & 0.00 & 0.00 & NA & NA \\
\hline 7:129929571 & rs377236326 & Exon & Nonsyn & 0.00 & 0.01 & -10.9 & $9.95 \times 10^{-1}$ & 0.00 & 0.00 & NA & NA \\
\hline $7: 129918048$ & & Intron & & 0.00 & 0.02 & -12.3 & $9.95 \times 10^{-1}$ & 0.00 & 0.00 & NA & NA \\
\hline $7: 129929365$ & rs377343685 & Intron & & 0.00 & 0.01 & -10.9 & $9.95 \times 10^{-1}$ & 0.00 & 0.00 & NA & NA \\
\hline 7:129921879 & & Intron & & 0.00 & 0.01 & -9.6 & $9.95 \times 10^{-1}$ & 0.00 & 0.00 & NA & NA \\
\hline
\end{tabular}


Table C-4. (Continued)

\begin{tabular}{|c|c|c|c|c|c|c|c|c|c|c|c|}
\hline \multirow[b]{2}{*}{ Chr:Loc ${ }^{a}$} & \multirow[b]{2}{*}{ RSID } & \multirow[b]{2}{*}{$\begin{array}{c}\text { Loca- } \\
\text { tion }\end{array}$} & \multirow[b]{2}{*}{ Function } & \multicolumn{4}{|c|}{ Cohort $(n=4217)$} & \multicolumn{4}{|c|}{ Case-control $(n=162)$} \\
\hline & & & & $\begin{array}{c}\text { MAF\% in } \\
\text { patients } \\
\text { with AP } \\
(n=97)\end{array}$ & $\begin{array}{c}\text { MAF\% in } \\
\text { patients } \\
\text { without } \\
\text { AP } \\
(n=3220)\end{array}$ & $\begin{array}{l}\text { Coeff- } \\
\text { icient }^{b}\end{array}$ & $P^{\mathbf{b}}$ & $\begin{array}{l}\text { MAF\% } \\
\text { in cases } \\
(n=47)\end{array}$ & $\begin{array}{c}\text { MAF\% in } \\
\text { controls } \\
(n=115)\end{array}$ & $\begin{array}{l}\text { Coeff- } \\
\text { icient }^{c}\end{array}$ & $P^{\mathbf{c}}$ \\
\hline $7: 129919250$ & & Intron & & 0.00 & 0.01 & -11.6 & $9.95 \times 10^{-1}$ & 0.00 & 0.00 & NA & $\mathrm{NA}$ \\
\hline 7:129929127 & & Intron & & 0.00 & 0.01 & -11.5 & $9.95 \times 10^{-1}$ & 0.00 & 0.00 & NA & NA \\
\hline $7: 129918102$ & & Intron & & 0.00 & 0.01 & -11.6 & $9.95 \times 10^{-1}$ & 0.00 & 0.00 & NA & NA \\
\hline $7: 129928953$ & & Intron & & 0.00 & 0.01 & -10.8 & $9.95 \times 10^{-1}$ & 0.00 & 0.00 & NA & NA \\
\hline $7: 129914304$ & & Intron & & 0.00 & 0.01 & -10.8 & $9.95 \times 10^{-1}$ & 0.00 & 0.00 & NA & NA \\
\hline $7: 129909748$ & & Intron & & 0.00 & 0.01 & -10.7 & $9.95 \times 10^{-1}$ & 0.00 & 0.00 & NA & NA \\
\hline $7: 129928717$ & & Intron & & 0.00 & 0.01 & -10.8 & $9.95 \times 10^{-1}$ & 0.00 & 0.00 & NA & NA \\
\hline $7: 129918631$ & & Intron & & 0.00 & 0.01 & -11.9 & $9.95 \times 10^{-1}$ & 0.00 & 0.00 & NA & NA \\
\hline 7:129913666 & & Intron & & 0.00 & 0.01 & -11.0 & $9.95 \times 10^{-1}$ & 0.00 & 0.00 & NA & NA \\
\hline $7: 129916523$ & & Exon & $\begin{array}{l}\text { Nonsyn } \\
\text { damaging }\end{array}$ & 0.00 & 0.01 & -10.8 & $9.95 \times 10^{-1}$ & 0.00 & 0.00 & NA & NA \\
\hline $7: 129912564$ & & Intron & & 0.00 & 0.01 & -10.5 & $9.95 \times 10^{-1}$ & 0.00 & 0.00 & NA & NA \\
\hline $7: 129916747$ & & Intron & & 0.00 & 0.02 & -12.3 & $9.95 \times 10^{-1}$ & 0.00 & 0.00 & NA & NA \\
\hline $7: 129913097$ & & Intron & & 0.00 & 0.01 & -10.8 & $9.95 \times 10^{-1}$ & 0.00 & 0.00 & NA & NA \\
\hline $7: 129914879$ & & Intron & & 0.00 & 0.01 & -10.7 & $9.95 \times 10^{-1}$ & 0.00 & 0.00 & NA & NA \\
\hline $7: 129930548$ & & $\begin{array}{l}\text { Down- } \\
\text { stream }\end{array}$ & & 0.00 & 12.50 & -16.7 & $9.95 \times 10^{-1}$ & NA & NA & NA & NA \\
\hline $7: 129930566$ & & $\begin{array}{l}\text { Down- } \\
\text { stream }\end{array}$ & & 0.00 & 0.06 & -12.7 & $9.95 \times 10^{-1}$ & 0.00 & 0.00 & NA & NA \\
\hline $7: 129930598$ & & $\begin{array}{l}\text { Down- } \\
\text { stream }\end{array}$ & & 0.00 & 0.01 & -9.9 & $9.95 \times 10^{-1}$ & 0.00 & 0.00 & NA & NA \\
\hline $7: 129918332$ & & Intron & & 0.00 & 0.01 & -10.8 & $9.96 \times 10^{-1}$ & 0.00 & 0.00 & NA & NA \\
\hline $7: 129915065$ & & Exon & $\begin{array}{l}\text { Nonsyn } \\
\text { damaging }\end{array}$ & 0.00 & 0.01 & -10.8 & $9.96 \times 10^{-1}$ & 0.00 & 0.00 & NA & NA \\
\hline $7: 129914022$ & & Intron & & 0.00 & 0.01 & -10.9 & $9.96 \times 10^{-1}$ & 0.00 & 0.00 & NA & NA \\
\hline $7: 129913438$ & & Intron & & 0.00 & 0.01 & -10.8 & $9.96 \times 10^{-1}$ & 0.00 & 0.00 & NA & NA \\
\hline
\end{tabular}


Table C-4. (Continued)

\begin{tabular}{|c|c|c|c|c|c|c|c|c|c|c|c|}
\hline \multirow[b]{2}{*}{ Chr:Loc ${ }^{a}$} & \multirow[b]{2}{*}{ RSID } & \multirow[b]{2}{*}{$\begin{array}{c}\text { Loca- } \\
\text { tion }\end{array}$} & \multirow[b]{2}{*}{ Function } & \multicolumn{4}{|c|}{ Cohort $(n=4217)$} & \multicolumn{4}{|c|}{ Case-control $(n=162)$} \\
\hline & & & & $\begin{array}{c}\text { MAF\% in } \\
\text { patients } \\
\text { with AP } \\
(n=97)\end{array}$ & $\begin{array}{c}\text { MAF\% in } \\
\text { patients } \\
\text { without } \\
\text { AP } \\
(n=3220) \\
\end{array}$ & $\begin{array}{l}\text { Coeff- } \\
\text { icient }^{b}\end{array}$ & $P^{\mathbf{b}}$ & $\begin{array}{l}\text { MAF\% } \\
\text { in cases } \\
(n=47)\end{array}$ & $\begin{array}{c}\text { MAF\% in } \\
\text { controls } \\
(n=115)\end{array}$ & $\begin{array}{l}\text { Coeff- } \\
\text { icient }^{c}\end{array}$ & $P^{\mathbf{c}}$ \\
\hline $7: 129916260$ & & Intron & & 0.00 & 0.01 & -10.6 & $9.96 \times 10^{-1}$ & 0.00 & 0.00 & $\mathrm{NA}$ & NA \\
\hline $7: 129921118$ & & Intron & & 0.00 & 0.01 & -11.8 & $9.96 \times 10^{-1}$ & 0.00 & 0.00 & NA & NA \\
\hline $7: 129916148$ & & Intron & & 0.00 & 0.01 & -11.6 & $9.96 \times 10^{-1}$ & 0.00 & 0.00 & NA & NA \\
\hline $7: 129912591$ & & Intron & & 0.00 & 0.01 & -10.9 & $9.96 \times 10^{-1}$ & 0.00 & 0.00 & NA & NA \\
\hline $7: 129909733$ & rs3744533 & Intron & & 0.00 & 0.02 & -12.1 & $9.96 \times 10^{-1}$ & 0.00 & 0.00 & NA & NA \\
\hline $7: 129919380$ & rs369196272 & Exon & Nonsyn & 0.00 & 0.02 & -12.3 & $9.96 \times 10^{-1}$ & 0.00 & 0.00 & NA & NA \\
\hline $7: 129914926$ & & Intron & & 0.00 & 0.01 & -10.9 & $9.96 \times 10^{-1}$ & 0.00 & 0.00 & NA & NA \\
\hline $7: 129917261$ & & Intron & & 0.00 & 0.01 & -11.2 & $9.96 \times 10^{-1}$ & 0.00 & 0.00 & NA & NA \\
\hline $7: 129916891$ & & Intron & & 0.00 & 0.01 & -10.6 & $9.96 \times 10^{-1}$ & 0.00 & 0.00 & NA & NA \\
\hline $7: 129917257$ & rs11485895 & Intron & & 0.00 & 0.01 & -10.6 & $9.96 \times 10^{-1}$ & 0.00 & 0.00 & NA & NA \\
\hline $7: 129914335$ & & Intron & & 0.00 & 0.04 & -13.8 & $9.96 \times 10^{-1}$ & 0.00 & 0.00 & NA & NA \\
\hline $7: 129910659$ & & Intron & & 0.00 & 0.01 & -10.5 & $9.96 \times 10^{-1}$ & 0.00 & 0.00 & NA & NA \\
\hline $7: 129906881$ & & Intron & & 0.00 & 0.01 & -10.5 & $9.96 \times 10^{-1}$ & 0.00 & 0.00 & NA & NA \\
\hline $7: 129928904$ & & Intron & & 0.00 & 0.01 & -11.9 & $9.96 \times 10^{-1}$ & 0.00 & 0.00 & NA & NA \\
\hline $7: 129919383$ & & Exon & $\begin{array}{c}\text { Nonsyn } \\
\text { damaging }\end{array}$ & 0.00 & 0.02 & -13.1 & $9.96 \times 10^{-1}$ & 0.00 & 0.00 & NA & NA \\
\hline $7: 129922108$ & rs139699182 & Intron & & 0.00 & 0.01 & -9.3 & $9.96 \times 10^{-1}$ & 0.00 & 0.00 & NA & NA \\
\hline $7: 129909634$ & & Exon & Nonsyn & 0.00 & 0.01 & -10.5 & $9.96 \times 10^{-1}$ & 0.00 & 0.00 & NA & NA \\
\hline $7: 129909459$ & & Intron & & 0.00 & 0.01 & -10.4 & $9.96 \times 10^{-1}$ & 0.00 & 0.00 & NA & NA \\
\hline $7: 129913672$ & & Intron & & 0.00 & 0.01 & -10.7 & $9.96 \times 10^{-1}$ & 0.00 & 0.00 & NA & NA \\
\hline $7: 129913034$ & & Intron & & 0.00 & 0.01 & -10.4 & $9.96 \times 10^{-1}$ & 0.00 & 0.00 & NA & NA \\
\hline $7: 129915808$ & & Intron & & 0.00 & 0.01 & -10.6 & $9.96 \times 10^{-1}$ & 0.00 & 0.00 & NA & NA \\
\hline $7: 129921430$ & & Intron & & 0.00 & 0.02 & -12.1 & $9.96 \times 10^{-1}$ & 0.00 & 0.00 & NA & NA \\
\hline $7: 129917371$ & & Intron & & 0.00 & 0.01 & -10.3 & $9.96 \times 10^{-1}$ & 0.00 & 0.00 & NA & NA \\
\hline $7: 129913346$ & & Intron & & 0.00 & 0.01 & -10.3 & $9.96 \times 10^{-1}$ & 0.00 & 0.00 & NA & NA \\
\hline
\end{tabular}


Table C-4. (Continued)

\begin{tabular}{|c|c|c|c|c|c|c|c|c|c|c|c|}
\hline \multirow[b]{2}{*}{ Chr:Loc ${ }^{a}$} & \multirow[b]{2}{*}{ RSID } & \multirow[b]{2}{*}{$\begin{array}{c}\text { Loca- } \\
\text { tion }\end{array}$} & \multirow[b]{2}{*}{ Function } & \multicolumn{4}{|c|}{ Cohort $(n=4217)$} & \multicolumn{4}{|c|}{ Case-control $(n=162)$} \\
\hline & & & & $\begin{array}{c}\text { MAF\% in } \\
\text { patients } \\
\text { with AP } \\
(n=97)\end{array}$ & $\begin{array}{l}\text { MAF\% in } \\
\text { patients } \\
\text { without } \\
\text { AP } \\
(n=3220)\end{array}$ & $\begin{array}{l}\text { Coeff- } \\
\text { icient }^{b}\end{array}$ & $P^{\mathbf{b}}$ & $\begin{array}{l}\text { MAF\% } \\
\text { in cases } \\
(n=47)\end{array}$ & $\begin{array}{c}\mathrm{MAF} \% \text { in } \\
\text { controls } \\
(\mathrm{n}=115)\end{array}$ & $\begin{array}{l}\text { Coeff- } \\
\text { icient }^{c}\end{array}$ & $P^{\mathbf{c}}$ \\
\hline $7: 129913524$ & & Intron & & 0.00 & 0.01 & -10.3 & $9.96 \times 10^{-1}$ & 0.00 & 0.00 & NA & $\mathrm{NA}$ \\
\hline $7: 129912495$ & & Intron & & 0.00 & 0.01 & -10.3 & $9.96 \times 10^{-1}$ & 0.00 & 0.00 & NA & NA \\
\hline $7: 129918785$ & & Intron & & 0.00 & 0.01 & -10.3 & $9.96 \times 10^{-1}$ & 0.00 & 0.00 & NA & NA \\
\hline $7: 129906818$ & rs 112731688 & Intron & & 0.00 & 0.01 & -10.3 & $9.96 \times 10^{-1}$ & 0.00 & 0.00 & NA & NA \\
\hline $7: 129917401$ & & Intron & & 0.00 & 0.01 & -10.3 & $9.96 \times 10^{-1}$ & 0.00 & 0.00 & NA & NA \\
\hline $7: 129929719$ & & $\begin{array}{l}\text { Down- } \\
\text { stream }\end{array}$ & & 0.00 & 0.01 & -10.7 & $9.96 \times 10^{-1}$ & 0.00 & 0.00 & NA & NA \\
\hline $7: 129917255$ & & Intron & & 0.00 & 0.02 & -13.0 & $9.96 \times 10^{-1}$ & 0.00 & 0.00 & NA & NA \\
\hline 7:129917279 & & Intron & & 0.00 & 0.02 & -13.0 & $9.96 \times 10^{-1}$ & 0.00 & 0.00 & NA & NA \\
\hline $7: 129918659$ & & Intron & & 0.00 & 0.01 & -11.0 & $9.96 \times 10^{-1}$ & 0.00 & 0.00 & NA & NA \\
\hline $7: 129917317$ & & Intron & & 0.00 & 0.01 & -11.9 & $9.96 \times 10^{-1}$ & 0.00 & 0.00 & NA & NA \\
\hline $7: 129921307$ & & Intron & & 0.00 & 0.01 & -10.6 & $9.96 \times 10^{-1}$ & 0.00 & 0.00 & NA & NA \\
\hline $7: 129916538$ & rs2188138 & Exon & $\begin{array}{l}\text { Nonsyn } \\
\text { damaging }\end{array}$ & 0.00 & 0.02 & -12.4 & $9.96 \times 10^{-1}$ & 0.00 & 0.00 & NA & NA \\
\hline 7:129929119 & & Intron & & 0.00 & 0.01 & -11.2 & $9.96 \times 10^{-1}$ & 0.00 & 0.00 & NA & NA \\
\hline $7: 129916215$ & & Intron & & 0.00 & 0.01 & -10.6 & $9.96 \times 10^{-1}$ & 0.00 & 0.00 & NA & NA \\
\hline $7: 129928968$ & & Intron & & 0.00 & 0.02 & -12.6 & $9.96 \times 10^{-1}$ & 0.00 & 0.00 & NA & NA \\
\hline $7: 129913793$ & & Intron & & 0.00 & 0.01 & -11.9 & $9.96 \times 10^{-1}$ & 0.00 & 0.00 & NA & NA \\
\hline $7: 129906635$ & & $\begin{array}{c}\text { Up- } \\
\text { stream }\end{array}$ & & 0.00 & 0.01 & -10.1 & $9.96 \times 10^{-1}$ & 0.00 & 0.00 & NA & NA \\
\hline $7: 129913545$ & & Intron & & 0.00 & 0.03 & -12.2 & $9.96 \times 10^{-1}$ & 0.00 & 0.45 & -13.4 & 0.988 \\
\hline $7: 129908764$ & & Intron & & 0.00 & 0.01 & -10.1 & $9.96 \times 10^{-1}$ & 0.00 & 0.00 & NA & NA \\
\hline $7: 129918370$ & & Intron & & 0.00 & 0.01 & -10.1 & $9.96 \times 10^{-1}$ & 0.00 & 0.00 & NA & NA \\
\hline 7:129917990 & & Intron & & 0.00 & 0.01 & -10.1 & $9.96 \times 10^{-1}$ & 0.00 & 0.00 & NA & NA \\
\hline $7: 129913518$ & & Intron & & 0.00 & 0.06 & -13.3 & $9.96 \times 10^{-1}$ & 0.00 & 1.69 & -15.9 & 0.993 \\
\hline
\end{tabular}


Table C-4. (Continued)

\begin{tabular}{|c|c|c|c|c|c|c|c|c|c|c|c|}
\hline \multirow[b]{2}{*}{ Chr:Loc ${ }^{a}$} & \multirow[b]{2}{*}{ RSID } & \multirow[b]{2}{*}{$\begin{array}{c}\text { Loca- } \\
\text { tion }\end{array}$} & \multirow[b]{2}{*}{ Function } & \multicolumn{4}{|c|}{ Cohort $(n=4217)$} & \multicolumn{4}{|c|}{ Case-control $(n=162)$} \\
\hline & & & & $\begin{array}{c}\text { MAF\% in } \\
\text { patients } \\
\text { with AP } \\
(n=97)\end{array}$ & $\begin{array}{c}\text { MAF\% in } \\
\text { patients } \\
\text { without } \\
\text { AP } \\
(n=3220)\end{array}$ & $\begin{array}{l}\text { Coeff- } \\
\text { icient }^{b}\end{array}$ & $P^{\mathbf{b}}$ & $\begin{array}{l}\text { MAF\% } \\
\text { in cases } \\
(n=47)\end{array}$ & $\begin{array}{c}\text { MAF\% in } \\
\text { controls } \\
(n=115)\end{array}$ & $\begin{array}{l}\text { Coeff- } \\
\text { icient }^{c}\end{array}$ & $P^{\mathbf{c}}$ \\
\hline $7: 129913972$ & & Intron & & 0.00 & 0.01 & -10.8 & $9.96 \times 10^{-1}$ & 0.00 & 0.00 & NA & $\mathrm{NA}$ \\
\hline $7: 129914066$ & & Intron & & 0.00 & 0.01 & -10.0 & $9.96 \times 10^{-1}$ & 0.00 & 0.00 & NA & NA \\
\hline $7: 129914076$ & & Intron & & 0.00 & 0.01 & -8.6 & $9.96 \times 10^{-1}$ & 0.00 & 0.00 & NA & NA \\
\hline $7: 129916234$ & & Intron & & 0.00 & 0.01 & -9.9 & $9.96 \times 10^{-1}$ & 0.00 & 0.00 & NA & NA \\
\hline $7: 129913835$ & & Intron & & 0.00 & 0.02 & -11.5 & $9.96 \times 10^{-1}$ & 0.00 & 0.00 & NA & NA \\
\hline $7: 129913836$ & & Intron & & 0.00 & 0.02 & -11.5 & $9.96 \times 10^{-1}$ & 0.00 & 0.00 & NA & NA \\
\hline $7: 129912302$ & & Intron & & 0.00 & 0.01 & -8.8 & $9.96 \times 10^{-1}$ & 0.00 & 0.00 & NA & NA \\
\hline $7: 129928839$ & & Intron & & 0.00 & 0.01 & -9.9 & $9.96 \times 10^{-1}$ & 0.00 & 0.00 & NA & NA \\
\hline $7: 129919065$ & & Intron & & 0.00 & 0.01 & -10.8 & $9.96 \times 10^{-1}$ & 0.00 & 0.00 & NA & NA \\
\hline $7: 129912112$ & & Intron & & 0.00 & 0.01 & -10.5 & $9.96 \times 10^{-1}$ & 0.00 & 0.00 & NA & NA \\
\hline $7: 129921820$ & & Intron & & 0.00 & 0.01 & -9.9 & $9.96 \times 10^{-1}$ & 0.00 & 0.00 & NA & NA \\
\hline $7: 129919344$ & rs373354928 & Exon & $\begin{array}{l}\text { Nonsyn } \\
\text { damaging }\end{array}$ & 0.00 & 0.01 & -9.9 & $9.96 \times 10^{-1}$ & 0.00 & 0.00 & NA & NA \\
\hline $7: 129928846$ & & Intron & & 0.00 & 0.01 & -12.8 & $9.96 \times 10^{-1}$ & 0.00 & 0.00 & NA & NA \\
\hline $7: 129916807$ & & Intron & & 0.00 & 0.01 & -10.0 & $9.96 \times 10^{-1}$ & 0.00 & 0.00 & NA & NA \\
\hline $7: 129919464$ & & Exon & $\begin{array}{l}\text { Nonsyn } \\
\text { damaging }\end{array}$ & 0.00 & 0.01 & -9.8 & $9.96 \times 10^{-1}$ & 0.00 & 0.00 & NA & NA \\
\hline $7: 129906897$ & & Intron & & 0.00 & 0.01 & -9.8 & $9.96 \times 10^{-1}$ & 0.00 & 0.00 & NA & NA \\
\hline $7: 129913430$ & & Intron & & 0.00 & 0.01 & -9.8 & $9.96 \times 10^{-1}$ & 0.00 & 0.00 & NA & NA \\
\hline $7: 129916835$ & & Intron & & 0.00 & 0.01 & -9.8 & $9.96 \times 10^{-1}$ & 0.00 & 0.00 & NA & NA \\
\hline $7: 129913613$ & & Intron & & 0.00 & 0.01 & -10.7 & $9.96 \times 10^{-1}$ & 0.00 & 0.00 & NA & NA \\
\hline 7:129919063 & & Intron & & 0.00 & 0.01 & -9.8 & $9.96 \times 10^{-1}$ & 0.00 & 0.00 & NA & NA \\
\hline $7: 129912247$ & & Intron & & 0.00 & 0.01 & -11.6 & $9.96 \times 10^{-1}$ & 0.00 & 0.00 & NA & NA \\
\hline $7: 129921304$ & & Intron & & 0.00 & 0.01 & -9.8 & $9.96 \times 10^{-1}$ & 0.00 & 0.00 & NA & NA \\
\hline $7: 129910453$ & & Intron & & 0.00 & 0.01 & -9.8 & $9.96 \times 10^{-1}$ & 0.00 & 0.00 & NA & NA \\
\hline
\end{tabular}


Table C-4. (Continued)

\begin{tabular}{|c|c|c|c|c|c|c|c|c|c|c|c|}
\hline \multirow[b]{2}{*}{ Chr:Loc ${ }^{a}$} & \multirow[b]{2}{*}{ RSID } & \multirow[b]{2}{*}{$\begin{array}{c}\text { Loca- } \\
\text { tion }\end{array}$} & \multirow[b]{2}{*}{ Function } & \multicolumn{4}{|c|}{ Cohort $(n=4217)$} & \multicolumn{4}{|c|}{ Case-control $(n=162)$} \\
\hline & & & & $\begin{array}{c}\text { MAF\% in } \\
\text { patients } \\
\text { with AP } \\
(n=97)\end{array}$ & $\begin{array}{c}\text { MAF\% in } \\
\text { patients } \\
\text { without } \\
\text { AP } \\
(n=3220)\end{array}$ & $\begin{array}{c}\text { Coef- } \\
\text { ficient }^{b}\end{array}$ & $P^{\mathbf{b}}$ & $\begin{array}{l}\text { MAF\% } \\
\text { in cases } \\
(n=47)\end{array}$ & $\begin{array}{c}\text { MAF\% in } \\
\text { controls } \\
(n=115)\end{array}$ & $\begin{array}{c}\text { Coef- } \\
\text { ficient }^{c}\end{array}$ & $P^{\mathbf{c}}$ \\
\hline $7: 129929093$ & & Intron & & 0.00 & 0.01 & -9.8 & $9.96 \times 10^{-1}$ & 0.00 & 0.00 & NA & $\mathrm{NA}$ \\
\hline $7: 129915269$ & & Intron & & 0.00 & 0.01 & -9.9 & $9.96 \times 10^{-1}$ & 0.00 & 0.00 & NA & NA \\
\hline $7: 129912403$ & & Intron & & 0.00 & 0.01 & -9.8 & $9.96 \times 10^{-1}$ & 0.00 & 0.00 & NA & NA \\
\hline 7:129913332 & & Intron & & 0.00 & 0.01 & -10.1 & $9.96 \times 10^{-1}$ & 0.00 & 0.00 & NA & NA \\
\hline $7: 129919202$ & & Intron & & 0.00 & 0.02 & -11.4 & $9.96 \times 10^{-1}$ & 0.00 & 0.00 & NA & NA \\
\hline $7: 129929387$ & rs3748345 & Intron & & 0.00 & 0.01 & -9.9 & $9.96 \times 10^{-1}$ & 0.00 & 0.00 & NA & NA \\
\hline $7: 129913096$ & & Intron & & 0.00 & 0.01 & -9.7 & $9.96 \times 10^{-1}$ & 0.00 & 0.00 & NA & NA \\
\hline $7: 129910480$ & & Intron & & 0.00 & 0.01 & -9.7 & $9.96 \times 10^{-1}$ & 0.00 & 0.00 & NA & NA \\
\hline $7: 129915155$ & & Intron & & 0.00 & 0.01 & -10.6 & $9.96 \times 10^{-1}$ & 0.00 & 0.00 & NA & NA \\
\hline 7:129919227 & & Intron & & 0.00 & 0.01 & -9.7 & $9.96 \times 10^{-1}$ & 0.00 & 0.00 & NA & NA \\
\hline 7:129912293 & & Intron & & 0.00 & 0.01 & -9.6 & $9.96 \times 10^{-1}$ & 0.00 & 0.00 & NA & NA \\
\hline 7:129918006 & & Intron & & 0.00 & 0.01 & -9.8 & $9.96 \times 10^{-1}$ & 0.00 & 0.00 & NA & NA \\
\hline $7: 129909638$ & & Exon & $\begin{array}{l}\text { Nonsyn } \\
\text { damaging }\end{array}$ & 0.00 & 0.01 & -9.7 & $9.96 \times 10^{-1}$ & 0.00 & 0.00 & NA & NA \\
\hline $7: 129912645$ & & Intron & & 0.00 & 0.01 & -9.7 & $9.96 \times 10^{-1}$ & 0.00 & 0.00 & NA & NA \\
\hline 7:129909569 & & Exon & Nonsyn & 0.00 & 0.01 & -9.7 & $9.96 \times 10^{-1}$ & 0.00 & 0.00 & NA & NA \\
\hline $7: 129916448$ & & Intron & & 0.00 & 0.01 & -9.9 & $9.96 \times 10^{-1}$ & 0.00 & 0.00 & NA & NA \\
\hline $7: 129908748$ & & Intron & & 0.00 & 0.01 & -11.9 & $9.96 \times 10^{-1}$ & 0.00 & 0.00 & NA & NA \\
\hline 7:129917688 & & Exon & $\begin{array}{l}\text { Nonsyn } \\
\text { damaging }\end{array}$ & 0.00 & 0.02 & -10.9 & $9.96 \times 10^{-1}$ & 0.00 & 0.00 & NA & NA \\
\hline $7: 129918230$ & & Intron & & 0.00 & 0.01 & -9.6 & $9.96 \times 10^{-1}$ & 0.00 & 0.00 & NA & NA \\
\hline 7:129914081 & & Intron & & 0.00 & 0.01 & -11.0 & $9.96 \times 10^{-1}$ & 0.00 & 0.00 & NA & NA \\
\hline 7:129914082 & rs6974812 & Intron & & 0.00 & 0.01 & -11.0 & $9.96 \times 10^{-1}$ & 0.00 & 0.00 & NA & NA \\
\hline 7:129914204 & & Intron & & 0.00 & 0.01 & -11.0 & $9.96 \times 10^{-1}$ & 0.00 & 0.00 & NA & NA \\
\hline $7: 129913687$ & & Intron & & 0.00 & 0.01 & -9.7 & $9.96 \times 10^{-1}$ & 0.00 & 0.00 & NA & NA \\
\hline
\end{tabular}


Table C-4. (Continued)

\begin{tabular}{|c|c|c|c|c|c|c|c|c|c|c|c|}
\hline \multirow[b]{2}{*}{ Chr:Loc ${ }^{a}$} & \multirow[b]{2}{*}{ RSID } & \multirow[b]{2}{*}{$\begin{array}{c}\text { Loca- } \\
\text { tion }\end{array}$} & \multirow[b]{2}{*}{ Function } & \multicolumn{4}{|c|}{ Cohort $(n=4217)$} & \multicolumn{4}{|c|}{ Case-control $(n=162)$} \\
\hline & & & & $\begin{array}{c}\text { MAF\% in } \\
\text { patients } \\
\text { with AP } \\
(n=97)\end{array}$ & $\begin{array}{c}\text { MAF\% in } \\
\text { patients } \\
\text { without } \\
\text { AP } \\
(n=3220)\end{array}$ & $\begin{array}{l}\text { Coeff- } \\
\text { icient }^{b}\end{array}$ & $P^{\mathbf{b}}$ & $\begin{array}{l}\text { MAF\% } \\
\text { in cases } \\
(n=47)\end{array}$ & $\begin{array}{c}\text { MAF\% in } \\
\text { controls } \\
(n=115)\end{array}$ & $\begin{array}{l}\text { Coeff- } \\
\text { icient }^{c}\end{array}$ & $P^{\mathbf{c}}$ \\
\hline $7: 129913525$ & & Intron & & 0.00 & 0.01 & -9.4 & $9.96 \times 10^{-1}$ & 0.00 & 0.00 & NA & $\mathrm{NA}$ \\
\hline 7:129915520 & & Intron & & 0.00 & 0.01 & -9.5 & $9.96 \times 10^{-1}$ & 0.00 & 0.00 & NA & NA \\
\hline 7:129930622 & & $\begin{array}{l}\text { Down- } \\
\text { stream }\end{array}$ & & 0.00 & 0.01 & -10.7 & $9.96 \times 10^{-1}$ & 0.00 & 0.00 & NA & NA \\
\hline $7: 129930565$ & & $\begin{array}{l}\text { Down- } \\
\text { stream }\end{array}$ & & 0.00 & 0.11 & -13.5 & $9.97 \times 10^{-1}$ & 0.00 & 0.00 & NA & NA \\
\hline 7:129922141 & & Intron & & 0.00 & 0.01 & -10.5 & $9.97 \times 10^{-1}$ & 0.00 & 0.00 & NA & NA \\
\hline 7:129906671 & & $\begin{array}{c}\text { Up- } \\
\text { stream }\end{array}$ & & 0.00 & 0.00 & NA & NA & 0.00 & 0.00 & NA & NA \\
\hline 7:129906765 & & Exon & Nonsyn & 0.00 & 0.00 & NA & NA & 0.00 & 0.43 & -13.4 & 0.988 \\
\hline 7:129906905 & & Intron & & 0.00 & 0.00 & NA & NA & 0.00 & 0.00 & NA & NA \\
\hline $7: 129908688$ & & Intron & & 0.00 & 0.00 & NA & NA & 0.00 & 0.43 & -14.1 & 0.987 \\
\hline 7:129908801 & & Exon & $\begin{array}{l}\text { Nonsyn } \\
\text { damaging }\end{array}$ & 0.00 & 0.00 & NA & NA & 0.00 & 0.00 & NA & NA \\
\hline 7:129910414 & & Intron & & 0.00 & 0.00 & NA & NA & 0.00 & 0.00 & NA & NA \\
\hline 7:129912126 & & Intron & & 0.00 & 0.00 & NA & NA & 0.00 & 0.00 & NA & NA \\
\hline 7:129912376 & & Intron & & 0.00 & 0.00 & NA & NA & 0.00 & 0.00 & NA & NA \\
\hline 7:129912718 & & Intron & & 0.00 & 0.00 & NA & NA & 1.06 & 0.00 & 15 & 0.986 \\
\hline $7: 129912837$ & & Intron & & 0.00 & 0.00 & NA & NA & 0.00 & 0.00 & NA & NA \\
\hline 7:129912877 & & Intron & & 0.00 & 0.00 & NA & NA & 0.00 & 0.43 & -13.3 & 0.988 \\
\hline 7:129913382 & & Intron & & 0.00 & 0.00 & NA & NA & 0.00 & 0.00 & NA & NA \\
\hline 7:129913440 & & Intron & & 0.00 & 0.00 & NA & NA & 0.00 & 0.00 & NA & NA \\
\hline 7:129913596 & & Intron & & 0.00 & 0.00 & NA & NA & 0.00 & 0.43 & -13.4 & 0.988 \\
\hline 7:129914063 & & Intron & & 0.00 & 0.00 & NA & NA & 0.00 & 0.00 & NA & NA \\
\hline 7:129915322 & & Intron & & 0.00 & 0.00 & NA & NA & 0.00 & 0.00 & NA & NA \\
\hline 7:129915498 & & Intron & & 0.00 & 0.00 & NA & NA & 0.00 & 0.43 & -14.1 & 0.987 \\
\hline
\end{tabular}


Table C-4. (Continued)

\begin{tabular}{|c|c|c|c|c|c|c|c|c|c|c|c|}
\hline \multirow[b]{2}{*}{ Chr:Loc ${ }^{a}$} & \multirow[b]{2}{*}{ RSID } & \multirow[b]{2}{*}{$\begin{array}{c}\text { Loca- } \\
\text { tion }\end{array}$} & \multirow[b]{2}{*}{ Function } & \multicolumn{4}{|c|}{ Cohort $(n=4217)$} & \multicolumn{4}{|c|}{ Case-control $(n=162)$} \\
\hline & & & & $\begin{array}{c}\text { MAF\% in } \\
\text { patients } \\
\text { with AP } \\
(n=97)\end{array}$ & $\begin{array}{l}\text { MAF\% in } \\
\text { patients } \\
\text { without } \\
\text { AP } \\
(n=3220) \\
\end{array}$ & $\begin{array}{l}\text { Coeff- } \\
\text { icient }^{b}\end{array}$ & $P^{\mathbf{b}}$ & $\begin{array}{l}\text { MAF\% } \\
\text { in cases } \\
(n=47)\end{array}$ & $\begin{array}{c}\text { MAF\% in } \\
\text { controls } \\
(n=115)\end{array}$ & $\begin{array}{l}\text { Coeff- } \\
\text { icient }^{c}\end{array}$ & $P^{\mathbf{c}}$ \\
\hline $7: 129915905$ & & Intron & & 0.00 & 0.00 & $\mathrm{NA}$ & NA & 0.00 & 0.00 & $\mathrm{NA}$ & NA \\
\hline 7:129916210 & & Intron & & 0.00 & 0.00 & NA & NA & 0.00 & 0.00 & NA & NA \\
\hline $7: 129916765$ & & Intron & & 0.00 & 0.00 & NA & NA & 0.00 & 0.00 & NA & NA \\
\hline $7: 129917055$ & & Intron & & 0.00 & 0.01 & NA & NA & 0.00 & 0.00 & NA & NA \\
\hline 7:129917213 & & Intron & & 0.00 & 0.00 & NA & NA & 0.00 & 0.00 & NA & NA \\
\hline 7:129918695 & & Intron & & 0.00 & 0.00 & NA & NA & 0.00 & 0.00 & NA & NA \\
\hline $7: 129918843$ & & Intron & & 0.00 & 0.00 & NA & NA & 0.00 & 0.00 & NA & NA \\
\hline 7:129919122 & & Intron & & 0.00 & 0.00 & NA & NA & 0.00 & 0.00 & NA & NA \\
\hline 7:129921333 & & Intron & & 0.00 & 0.00 & NA & NA & 0.00 & 0.00 & NA & NA \\
\hline 7:129921880 & & Intron & & 0.52 & 0.00 & NA & NA & 0.00 & 0.00 & NA & NA \\
\hline $7: 129929508$ & & Exon & Nonsyn & 0.00 & 0.00 & NA & NA & 0.00 & 0.43 & -13.4 & 0.988 \\
\hline
\end{tabular}

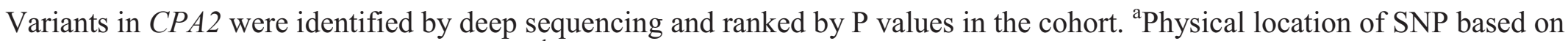
Feb 2009 human genome assembly (hg19). ${ }^{\mathrm{b}}$ Coefficient and P value of minor alleles were estimated using Cox proportional hazard model. ${ }^{\mathrm{C}}$ Coefficient and $\mathrm{P}$ value of minor alleles were estimated using logistic regression model. P values less than 0.05 were highlighted. MAF, minor allele frequency. Chr, chromosome. Syn, synonymous. Nonsyn, nonsynonymous. UTR, untranslated region. NA, not available. 
Table C-5. Top genes $(P<0.05)$ in the gene-level analysis of nonsense variants identified in putative pancreatitis $(n=$ 42) and non-pancreatitis genes $(n=241)$ based on variants identified by deep sequencing

\begin{tabular}{|c|c|c|c|c|c|}
\hline $\operatorname{Rank}^{\mathrm{a}}$ & Gene & N of SNPs ${ }^{b}$ & $\begin{array}{c}\text { Cohort SKAT } P \\
(n=4217)\end{array}$ & $\begin{array}{c}\text { Case-control SKAT } P \\
(\mathrm{n}=162)\end{array}$ & Pancreatitis-associated $^{\mathrm{c}}$ \\
\hline 1 & HOGA1 & 1 & $6.37 \times 10^{-11}$ & - & Yes \\
\hline 2 & $C P A 2$ & 1 & $6.69 \times 10^{-11}$ & 0.03 & Yes \\
\hline 3 & ZNF233 & 4 & $9.90 \times 10^{-9}$ & 0.05 & Yes \\
\hline 4 & C9orf117 & 2 & $4.83 \times 10^{-8}$ & - & Yes \\
\hline 5 & LRRC39 & 1 & $2.48 \times 10^{-6}$ & - & Yes \\
\hline 6 & $X D H$ & 1 & $6.40 \times 10^{-6}$ & - & No \\
\hline 7 & $X R C C 1$ & 5 & $1.41 \times 10^{-5}$ & - & No \\
\hline 8 & IFNA5 & 1 & $2.28 \times 10^{-5}$ & - & Yes \\
\hline 9 & $Q P C T$ & 3 & $3.87 \times 10^{-4}$ & 0.73 & No \\
\hline 10 & C11orf63 & 1 & $4.72 \times 10^{-4}$ & - & Yes \\
\hline 11 & ZRANB3 & 1 & $6.79 \times 10^{-4}$ & - & Yes \\
\hline 12 & C12orf40 & 1 & $2.67 \times 10^{-3}$ & - & Yes \\
\hline 13 & $D D X 49$ & 3 & $3.85 \times 10^{-3}$ & 0.72 & Yes \\
\hline 14 & $G C K R$ & 2 & $4.24 \times 10^{-3}$ & 0.07 & Yes \\
\hline 15 & SNTG2 & 1 & $5.10 \times 10^{-3}$ & - & Yes \\
\hline 16 & RUFY4 & 2 & $9.16 \times 10^{-3}$ & - & Yes \\
\hline 17 & MPZL3 & 1 & $9.40 \times 10^{-3}$ & - & Yes \\
\hline 18 & $D A P L 1$ & 2 & $1.36 \times 10^{-2}$ & - & No \\
\hline 19 & $A L S 2 C L$ & 1 & $1.43 \times 10^{-2}$ & - & Yes \\
\hline 20 & CENPQ & 1 & $1.55 \times 10^{-2}$ & 0.73 & No \\
\hline 21 & $N M I$ & 3 & $2.51 \times 10^{-2}$ & - & Yes \\
\hline
\end{tabular}

${ }^{a}$ Rank of gene based on the cohort SKAT P-value. ${ }^{b}$ Number of SNPs in this category. ${ }^{c}$ Genes associated with pancreatitis (based on GWAS or literature). All in 10K, all SNPs within 10K region of the gene. 


\section{Top Networks}

ID Associated Network Functions

Score

1 Endocrine System Disorders, Gastrointestinal Disease, Hereditary Disorder 35

2 Cell Death and Survival, Cell-To-Cell Signaling and Interaction, Cell Cycle 21

3 Cellular Growth and Proliferation, Cell Cycle, Cell-To-Cell Signaling and Interaction 21

4 Cancer, Gastrointestinal Disease, Organismal Injury and Abnormalities 3

5 Hair and Skin Development and Function, Tissue Development, Hematological System Development and Function 3

Diseases and Disorders

Name

Endocrine System Disorders

Gastrointestinal Disease

Hereditary Disorder

Inflammatory Disease

Organismal Injury and Abnormalities p-value range

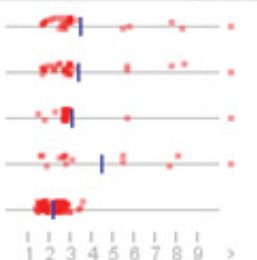

\# Molecules

$3.00 \mathrm{E}-02-1.12 \mathrm{E}-11 \quad 10$

$3.00 \mathrm{E}-02-1.12 \mathrm{E}-11 \quad 18$

4.28E-02-1.12E-11 8

$2.26 \mathrm{E}-02-1.12 \mathrm{E}-11 \quad 10$

$4.83 \mathrm{E}-02-2.30 \mathrm{E}-04 \quad 36$

Molecular and Cellular Functions

Name

$\mathrm{p}$-value range

Cellular Function and Maintenance

Molecular Transport

Cell Death and Survival

$4=$

4.83E-02 - 5.29E-04

\# Molecules

Fa

4.83E-02 - 5.29E-04

8

$+*$

$4.28 \mathrm{E}-02-1.53 \mathrm{E}-03$

$\% \quad 1.14 \mathrm{E}-02-1.90 \mathrm{E}-03$

$2.82 \mathrm{E}-02-1.90 \mathrm{E}-03$

$\rightarrow+$

$\begin{array}{llllllll}1 & 1 & 1 & 1 & 1 & 1 & 1 & 1 \\ 2 & 3 & 4 & 5 & 6 & 7 & 8 & 9\end{array} \quad>$

\section{0}

8

8

36

Amino Acid Metabolism

Physiological System Development and Function

Name

Digestive System Development and Function

Organ Morphology

Organismal Development

Cardiovascular System Development and Function

Cell-mediated Immune Response

\begin{tabular}{ccc}
\multicolumn{1}{c}{$p$} & \#-value range \\
$3.37 \mathrm{E}-02-1.39 \mathrm{E}-03$ & 4 \\
$4.47 \mathrm{E}-02-1.39 \mathrm{E}-03$ & 6 \\
$4.83 \mathrm{E}-02-1.39 \mathrm{E}-03$ & 8 \\
4. & $4.83 \mathrm{E}-02-1.90 \mathrm{E}-03$ & 2 \\
1 & $1.90 \mathrm{E}-03-1.90 \mathrm{E}-03$ & 1
\end{tabular}

Figure C-2. Top pathways and functions of pancreatitis-associated genes $(n=42)$ Pathway analysis of 42 pancreatitis-associated genes (see Table 4-3) was performed using Ingenuity. ${ }^{205}$ Scores of the networks indicated the relevance between networks and genes, and $\mathrm{P}$ values of the top diseases or functions were calculated using Fisher's exact test to determine the probability that the association between the genes in the data set and the function is due to chance alone. The numbers of genes in each functional category are shown. 


\begin{tabular}{lc}
\hline Top Networks & Score \\
\hline ID Associated Network Functions & 25 \\
\hline 1 Cell Cycle, Dermatological Diseases and Conditions, Developmental Disorder & 25 \\
2 Cellular Compromise, Cell Death and Survival, Cancer & 9 \\
3 Endocrine System Development and Function, Energy Production, Small Molecule Biochemistry & 3 \\
4 Cancer, Gastrointestinal Disease, Organismal Injury and Abnormalities & 3 \\
5 Hair and Skin Development and Function, Tissue Development, Hematological System Development and Function
\end{tabular}

Diseases and Disorders

\begin{tabular}{|c|c|c|c|}
\hline Name & $p-v$ & e range & \# Molecules \\
\hline Cancer & $\rightarrow$ & $4.58 \mathrm{E}-02-1.51 \mathrm{E}-03$ & 25 \\
\hline Developmental Disorder & $\mu$ & $1.95 \mathrm{E}-02-1.51 \mathrm{E}-03$ & 5 \\
\hline Gastrointestinal Disease & -14 & $2.39 \mathrm{E}-02-1.51 \mathrm{E}-03$ & 10 \\
\hline Hereditary Disorder & $r$ & $1.26 \mathrm{E}-02-1.51 \mathrm{E}-03$ & 3 \\
\hline Metabolic Disease & 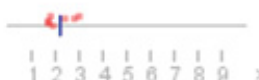 & $2.39 \mathrm{E}-02-1.51 \mathrm{E}-03$ & 4 \\
\hline
\end{tabular}

Molecular and Cellular Functions

\begin{tabular}{|c|c|c|c|}
\hline Name & \multicolumn{2}{|c|}{$\mathrm{p}$-value range } & \# Molecules \\
\hline Cell Death and Survival & -r. & $4.74 \mathrm{E}-02-7.79 \mathrm{E}-04$ & 4 \\
\hline Cell Cycle & $\rightarrow k$ & $2.24 \mathrm{E}-02-1.51 \mathrm{E}-03$ & 2 \\
\hline Cell-To-Cell Signaling and Interaction & $\rightarrow \because$ & 4.15E-02 - $1.51 \mathrm{E}-03$ & 4 \\
\hline Cellular Assembly and Organization & $-w$ & $4.44 \mathrm{E}-02-1.51 \mathrm{E}-03$ & 8 \\
\hline Cellular Growth and Proliferation & $\frac{7=}{7} \begin{array}{lllllllll}1 & 1 & 1 & 1 & 1 & 1 & 1 & 1 & 1 \\
1 & 2 & 3 & 4 & 5 & 6 & 7 & 8 & 9\end{array}$ & $3.86 \mathrm{E}-02-1.51 \mathrm{E}-03$ & 4 \\
\hline
\end{tabular}

Physiological System Development and Function

\begin{tabular}{|c|c|c|c|}
\hline Name & $p-v$ & e range & $=$ Molecules \\
\hline Cardiovascular System Development and Function & -1. & $3.86 \mathrm{E}-02-1.51 \mathrm{E}-03$ & 1 \\
\hline Cell-mediated Immune Response & -1 & $4.58 \mathrm{E}-02-1.51 \mathrm{E}-03$ & 2 \\
\hline Hematological System Development and Function & 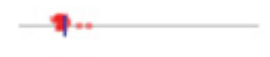 & $4.73 \mathrm{E}-02-1.51 \mathrm{E}-03$ & 5 \\
\hline Lymphoid Tissue Structure and Development & -1. & $4.58 \mathrm{E}-02-1.51 \mathrm{E}-03$ & 2 \\
\hline Organ Morphology & 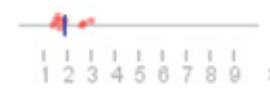 & $4.73 E-02-1.51 E-03$ & 2 \\
\hline
\end{tabular}

Figure C-3. Top pathways and functions of pancreatitis-associated genes identified by GWAS $(n=34)$

Pathway analysis of 34 pancreatitis-associated genes identified by GWAS (see Table 4-3) was performed using Ingenuity. ${ }^{205}$ Scores of the networks indicated the relevance between networks and genes, and P values of the top diseases or functions were calculated using Fisher's exact test to determine the probability that the association between the genes in the data set and the function is due to chance alone. The numbers of genes in each functional category are shown. 
Table C-6. SNPs $(n=81)$ identified by deep sequencing in previously reported pancreatitis-associated candidate genes $(\mathrm{n}=8)$ at the $P<0.05$ level (highlighted) in either the cohort or the case-control group

\begin{tabular}{|c|c|c|c|c|c|c|c|c|c|c|c|c|c|}
\hline \multirow[b]{2}{*}{$\begin{array}{c}\text { Gene } \\
(n \text { of } S N P s \text { at } \\
P<0.05 / n \text { of } \\
\text { all SNPs) }{ }^{\mathrm{a}}\end{array}$} & \multirow[b]{2}{*}{ Chr:Loc ${ }^{b}$} & \multirow[b]{2}{*}{ RSID } & \multirow[b]{2}{*}{ Function } & \multirow[b]{2}{*}{$\begin{array}{l}\text { Major } \\
\text { allele/ } \\
\text { minor } \\
\text { allele }\end{array}$} & \multicolumn{4}{|c|}{ Cohort $(n=4217)$} & \multicolumn{4}{|c|}{ Case-control $(n=162)$} & \multirow[b]{2}{*}{$\begin{array}{l}\text { Dire- } \\
\text { ction }^{\text {e }}\end{array}$} \\
\hline & & & & & $\begin{array}{c}\text { MAF\% } \\
\text { in } \\
\text { patients } \\
\text { with } \\
\text { AP } \\
(n=95)\end{array}$ & $\begin{array}{c}\text { MAF\% } \\
\text { in } \\
\text { patients } \\
\text { without } \\
\text { AP } \\
(n=3161)\end{array}$ & $\begin{array}{l}\text { Coeff- } \\
\text { icient }^{c}\end{array}$ & $P^{c}$ & $\begin{array}{c}\text { MAF\% } \\
\text { in cases } \\
(\mathrm{n}= \\
47)\end{array}$ & $\begin{array}{c}\text { MAF\% } \\
\text { in con- } \\
\text { trols } \\
(\mathrm{n}= \\
115)\end{array}$ & $\begin{array}{l}\text { Coeff- } \\
\text { icient }^{d}\end{array}$ & $P^{\mathrm{d}}$ & \\
\hline \multirow{8}{*}{$\begin{array}{c}\text { CTRC }^{162-164} \\
(8 / 159)\end{array}$} & $1: 15766660$ & & Intronic & $\mathrm{C} / \mathrm{T}$ & 0.63 & 0.03 & 2.219 & $3.01 \times 10^{-2}$ & 0.00 & 0.00 & NA & NA & NA \\
\hline & $1: 15766722$ & & Intronic & $-/ \mathrm{C}$ & 1.05 & 0.08 & 2.991 & $4.48 \times 10^{-5}$ & 0.00 & 0.00 & NA & NA & NA \\
\hline & $1: 15766730$ & & Intronic & $\mathrm{T} / \mathrm{C}$ & 3.45 & 1.57 & 0.862 & $4.19 \times 10^{-2}$ & 0.00 & 0.00 & NA & NA & NA \\
\hline & 1:15766836 & & Syn & $\mathrm{C} / \mathrm{T}$ & 0.52 & 0.00 & 3.386 & $1.53 \times 10^{-3}$ & 0.00 & 0.00 & NA & NA & NA \\
\hline & $1: 15767036$ & rs49778 & Syn & $\mathrm{C} / \mathrm{T}$ & 7.37 & 9.99 & -0.385 & $1.81 \times 10^{-1}$ & 11.70 & 6.52 & 0.93 & 0.044 & - \\
\hline & $1: 15768845$ & & Intronic & $\mathrm{C} / \mathrm{A}$ & 0.52 & 0.01 & 3.147 & $2.26 \times 10^{-3}$ & 0.00 & 0.00 & NA & NA & NA \\
\hline & 1:15770102 & rs545634 & Intronic & $\mathrm{G} / \mathrm{A}$ & 6.70 & 9.69 & -0.343 & $2.36 \times 10^{-1}$ & 11.70 & 6.52 & 0.93 & 0.044 & - \\
\hline & $1: 15772962$ & & Intronic & $\mathrm{G} / \mathrm{A}$ & 0.54 & 0.01 & 3.545 & $5.11 \times 10^{-4}$ & 0.00 & 0.00 & NA & NA & NA \\
\hline \multirow{7}{*}{$\begin{array}{l}C A S R^{72} \\
(7 / 255)\end{array}$} & $3: 121902632$ & rs 188613418 & Upstream & $\mathrm{A} / \mathrm{G}$ & 0.51 & 0.00 & 3.316 & $1.33 \times 10^{-3}$ & 0.00 & 0.00 & NA & NA & NA \\
\hline & $3: 121952299$ & & Intronic & $\mathrm{C} / \mathrm{T}$ & 0.51 & 0.00 & 2.104 & $4.34 \times 10^{-2}$ & 0.00 & 0.00 & NA & NA & NA \\
\hline & $3: 121952353$ & rs 145416265 & Intronic & $\mathrm{T} / \mathrm{C}$ & 0.51 & 0.02 & 2.718 & $9.32 \times 10^{-3}$ & 0.00 & 0.00 & NA & NA & NA \\
\hline & $3: 121952524$ & & Intronic & $\mathrm{A} / \mathrm{G}$ & 0.51 & 0.02 & 2.318 & $2.46 \times 10^{-2}$ & 0.00 & 0.00 & NA & NA & NA \\
\hline & 3:121980909 & & Nonsyn & $\mathrm{G} / \mathrm{A}$ & 0.51 & 0.00 & 5.092 & $9.01 \times 10^{-7}$ & 0.00 & 0.00 & NA & NA & NA \\
\hline & $3: 122000815$ & & Intronic & $\mathrm{C} / \mathrm{T}$ & 0.51 & 0.00 & 4.559 & $1.12 \times 10^{-5}$ & 0.00 & 0.00 & NA & NA & NA \\
\hline & $3: 122000822$ & rs 113610526 & Intronic & $\mathrm{T} / \mathrm{G}$ & 0.51 & 0.01 & 3.576 & $4.81 \times 10^{-4}$ & 0.00 & 0.00 & NA & NA & NA \\
\hline \multirow{2}{*}{$\begin{array}{l}\text { SPINK } 1^{71,153,162} \\
(2 / 104)\end{array}$} & 5:147207668 & & Syn & $\mathrm{A} / \mathrm{G}$ & 0.52 & 0.01 & 3.217 & $1.50 \times 10^{-3}$ & 0.00 & 0.00 & NA & NA & NA \\
\hline & 5:147210229 & & Intronic & $\mathrm{T} / \mathrm{C}$ & 0.52 & 0.00 & 4.064 & $9.97 \times 10^{-5}$ & 0.00 & 0.00 & NA & NA & NA \\
\hline
\end{tabular}


Table C-6. (Continued)

\begin{tabular}{|c|c|c|c|c|c|c|c|c|c|c|c|c|c|}
\hline \multirow[b]{2}{*}{$\begin{array}{c}\text { Gene } \\
\text { (n of SNPs at } \\
P<0.05 / n \text { of } \\
\text { all SNPs) }^{\mathrm{a}}\end{array}$} & \multirow[b]{2}{*}{ Chr:Loc ${ }^{b}$} & \multirow[b]{2}{*}{ RSID } & \multirow[b]{2}{*}{ Function } & \multirow[b]{2}{*}{$\begin{array}{c}\text { Major } \\
\text { allele/ } \\
\text { minor } \\
\text { allele }\end{array}$} & \multicolumn{4}{|c|}{ Cohort $(n=4217)$} & \multicolumn{4}{|c|}{ Case-control $(n=162)$} & \multirow[b]{2}{*}{$\begin{array}{l}\text { Dire- } \\
\text { ction }^{\mathrm{e}}\end{array}$} \\
\hline & & & & & $\begin{array}{l}\text { MAF\% } \\
\text { in } \\
\text { patients } \\
\text { with AP } \\
(n=95)\end{array}$ & $\begin{array}{c}\text { MAF\% in } \\
\text { patients } \\
\text { without AP } \\
(n=3,161)\end{array}$ & $\begin{array}{l}\text { Coeff- } \\
\text { icient }^{\mathrm{c}}\end{array}$ & $P^{\mathbf{c}}$ & $\begin{array}{c}\text { MAF } \\
\% \text { in } \\
\text { cases } \\
(n= \\
47)\end{array}$ & $\begin{array}{c}\text { MAF } \\
\% \text { in } \\
\text { con- } \\
\text { trols } \\
(\mathrm{n}= \\
115)\end{array}$ & $\begin{array}{l}\text { Coeff- } \\
\text { icient }^{d}\end{array}$ & $P^{d}$ & \\
\hline \multirow{16}{*}{$\begin{array}{c}C_{T T R}^{153,162} \\
(32 / 535)\end{array}$} & $7: 117119516$ & & Upstream & $\mathrm{G} / \mathrm{A}$ & 0.52 & 0.00 & 6.333 & $4.40 \times 10^{-9}$ & 0.00 & 0.00 & NA & $\mathrm{NA}$ & NA \\
\hline & $7: 117119599$ & & Upstream & $\mathrm{G} / \mathrm{C}$ & 0.52 & 0.00 & 4.070 & $1.11 \times 10^{-4}$ & 0.00 & 0.00 & NA & NA & NA \\
\hline & $7: 117120323$ & & Intronic & $\mathrm{T} / \mathrm{C}$ & 0.52 & 0.00 & 5.175 & $1.35 \times 10^{-6}$ & 0.00 & 0.00 & NA & NA & NA \\
\hline & $7: 117120586$ & rs 1876659 & Intronic & $\mathrm{A} / \mathrm{G}$ & 0.52 & 0.01 & 3.273 & $1.64 \times 10^{-3}$ & 0.00 & 0.00 & NA & NA & NA \\
\hline & $7: 117120672$ & & Intronic & $\mathrm{A} / \mathrm{G}$ & 0.52 & 0.04 & 2.174 & $3.55 \times 10^{-2}$ & 0.00 & 0.00 & NA & NA & NA \\
\hline & $7: 117144420$ & & Intronic & $\mathrm{A} / \mathrm{G}$ & 0.52 & 0.00 & 3.601 & $6.92 \times 10^{-4}$ & 0.00 & 0.00 & NA & NA & NA \\
\hline & $7: 117171012$ & & Syn & $\mathrm{G} / \mathrm{A}$ & 0.52 & 0.00 & 3.601 & $6.92 \times 10^{-4}$ & 0.00 & 0.00 & NA & NA & NA \\
\hline & $7: 117174527$ & rs 15757811 & Intronic & $\mathrm{C} / \mathrm{G}$ & 0.54 & 0.01 & 3.626 & $4.34 \times 10^{-4}$ & 1.11 & 0.00 & 15.65 & 0.986 & + \\
\hline & $7: 117175372$ & rs1219946 & $\begin{array}{l}\text { Nonsyn } \\
\text { damaging }\end{array}$ & $\mathrm{A} / \mathrm{G}$ & 0.52 & 0.01 & 3.179 & $2.63 \times 10^{-3}$ & 0.00 & 0.00 & NA & NA & NA \\
\hline & $7: 117180324$ & rs77932196 & $\begin{array}{l}\text { Nonsyn } \\
\text { damaging }\end{array}$ & $\mathrm{G} / \mathrm{C}$ & 0.52 & 0.00 & 3.944 & $1.12 \times 10^{-4}$ & 0.00 & 0.00 & NA & NA & NA \\
\hline & $7: 117180338$ & rs193922497 & $\begin{array}{l}\text { Nonsyn } \\
\text { damaging }\end{array}$ & $\mathrm{C} / \mathrm{T}$ & 1.03 & 0.04 & 2.525 & $5.13 \times 10^{-4}$ & 0.00 & 0.00 & NA & NA & NA \\
\hline & $7: 117182242$ & & Intronic & $\mathrm{G} / \mathrm{A}$ & 0.54 & 0.00 & 2.782 & $5.92 \times 10^{-3}$ & 0.00 & 0.00 & NA & NA & NA \\
\hline & $7: 117188684$ & & Intronic & $-/ \mathrm{TT}$ & 1.11 & 1.02 & -0.531 & $5.98 \times 10^{-1}$ & 4.88 & 0.48 & 2.69 & 0.019 & - \\
\hline & $7: 117188736$ & rs4727853 & $\begin{array}{l}\text { Nonsyn } \\
\text { damaging }\end{array}$ & $\mathrm{C} / \mathrm{A}$ & 39.13 & 40.22 & -0.130 & $6.59 \times 10^{-1}$ & 31.94 & 41.46 & -1.01 & 0.032 & + \\
\hline & $7: 117188750$ & rs2188593 & $\begin{array}{l}\text { Nonsyn } \\
\text { damaging }\end{array}$ & $\mathrm{C} / \mathrm{T}$ & 12.96 & 14.23 & -0.131 & $6.12 \times 10^{-1}$ & 3.85 & 14.20 & -1.59 & 0.016 & + \\
\hline & 7:117188896 & & Intronic & $\mathrm{A} / \mathrm{G}$ & 0.52 & 0.00 & 4.070 & $1.11 \times 10^{-4}$ & 0.00 & 0.00 & NA & NA & NA \\
\hline
\end{tabular}


Table C-6. (Continued)

\begin{tabular}{|c|c|c|c|c|c|c|c|c|c|c|c|c|c|}
\hline \multirow[b]{2}{*}{$\begin{array}{c}\text { Gene } \\
(n \text { of SNPs at } \\
P<0.05 / n \text { of } \\
\text { all SNPs) }\end{array}$} & \multirow[b]{2}{*}{ Chr:Loc ${ }^{b}$} & \multirow[b]{2}{*}{ RSID } & \multirow[b]{2}{*}{ Function } & \multirow[b]{2}{*}{$\begin{array}{l}\text { Major } \\
\text { allele/ } \\
\text { minor } \\
\text { allele }\end{array}$} & \multicolumn{4}{|c|}{ Cohort $(n=4217)$} & \multicolumn{4}{|c|}{ Case-control $(n=162)$} & \multirow[b]{2}{*}{$\begin{array}{l}\text { Dire- } \\
\text { ction }^{\text {e }}\end{array}$} \\
\hline & & & & & $\begin{array}{l}\text { MAF\% } \\
\text { in } \\
\text { patients } \\
\text { with AP } \\
(n=95)\end{array}$ & $\begin{array}{c}\text { MAF\% in } \\
\text { patients } \\
\text { without AP } \\
(n=3,161)\end{array}$ & $\begin{array}{l}\text { Coeff- } \\
\text { icient }^{c}\end{array}$ & $P^{\mathrm{c}}$ & $\begin{array}{c}\text { MAF } \\
\% \text { in } \\
\text { cases } \\
(n= \\
47)\end{array}$ & $\begin{array}{l}\text { MAF } \\
\% \text { in } \\
\text { con- } \\
\text { trols } \\
(n= \\
115)\end{array}$ & $\begin{array}{l}\text { Coeff- } \\
\text { icient }^{d}\end{array}$ & $P^{d}$ & \\
\hline \multirow{16}{*}{$\begin{array}{c}C F T R^{153,162} \\
(32 / 535)\end{array}$} & $7: 117199641$ & rs1891 & $\begin{array}{c}\text { Nonsyn } \\
\text { damaging }\end{array}$ & $\mathrm{A} / \mathrm{G}$ & 0.52 & 0.02 & 3.483 & $5.85 \times 10^{-4}$ & 0.00 & 0.00 & NA & NA & NA \\
\hline & $7: 117199648$ & rs7457153 & $\begin{array}{l}\text { Nonsyn } \\
\text { damaging }\end{array}$ & $\mathrm{T} / \mathrm{G}$ & 0.54 & 0.07 & 2.365 & $1.97 \times 10^{-2}$ & 0.00 & 0.88 & -14.32 & 0.989 & - \\
\hline & $7: 117230283$ & rs1714229 & Intronic & $\mathrm{T} / \mathrm{C}$ & 9.24 & 3.16 & 0.819 & $1.12 \times 10^{-2}$ & 2.22 & 0.45 & 1.37 & 0.330 & + \\
\hline & $7: 117234884$ & & Intronic & $\mathrm{A} / \mathrm{G}$ & 0.53 & 0.00 & 3.909 & $1.25 \times 10^{-4}$ & 0.00 & 0.00 & NA & NA & NA \\
\hline & $7: 117242735$ & & Intronic & $\mathrm{G} / \mathrm{A}$ & 0.52 & 0.00 & 3.583 & $6.37 \times 10^{-4}$ & 0.00 & 0.00 & NA & NA & NA \\
\hline & $7: 117242753$ & & Intronic & $\mathrm{G} / \mathrm{A}$ & 0.52 & 0.00 & 5.333 & $1.24 \times 10^{-4}$ & 0.00 & 0.00 & NA & NA & NA \\
\hline & $7: 117243663$ & rs1219934 & Nonsyn & $\mathrm{C} / \mathrm{T}$ & 0.52 & 0.02 & 2.852 & $5.08 \times 10^{-3}$ & 0.00 & 0.00 & NA & NA & NA \\
\hline & $7: 117243784$ & & $\begin{array}{l}\text { Nonsyn } \\
\text { damaging }\end{array}$ & $\mathrm{G} / \mathrm{A}$ & 0.52 & 0.00 & 2.214 & $3.41 \times 10^{-2}$ & 0.00 & 0.00 & NA & NA & NA \\
\hline & $7: 117250493$ & & Intronic & G/A & 4.35 & 1.57 & 0.999 & $2.01 \times 10^{-2}$ & 0.00 & 2.33 & -15.34 & 0.990 & - \\
\hline & $7: 117250824$ & & Intronic & $\mathrm{C} / \mathrm{G}$ & 0.52 & 0.01 & 2.598 & $1.01 \times 10^{-2}$ & 0.00 & 0.00 & NA & NA & NA \\
\hline & $7: 117251794$ & & $\begin{array}{l}\text { Nonsyn } \\
\text { damaging }\end{array}$ & $\mathrm{A} / \mathrm{C}$ & 0.52 & 0.00 & 3.696 & $3.48 \times 10^{-4}$ & 0.00 & 0.00 & NA & NA & NA \\
\hline & $7: 117254753$ & & $\begin{array}{l}\text { Nonsyn } \\
\text { damaging }\end{array}$ & $\mathrm{G} / \mathrm{C}$ & 0.52 & 0.05 & 2.065 & $4.02 \times 10^{-2}$ & 0.00 & 0.00 & NA & NA & NA \\
\hline & $7: 117282413$ & & Intronic & $\mathrm{T} / \mathrm{C}$ & 0.52 & 0.04 & 2.111 & $3.62 \times 10^{-2}$ & 0.00 & 0.00 & NA & NA & NA \\
\hline & $7: 117282716$ & & Intronic & $\mathrm{A} / \mathrm{C}$ & 0.52 & 0.00 & 4.264 & $4.50 \times 10^{-5}$ & 0.00 & 0.00 & NA & NA & NA \\
\hline & $7: 117307164$ & rs1591472 & Down-stream & $\mathrm{G} / \mathrm{A}$ & 0.52 & 0.01 & 3.180 & $2.21 \times 10^{-3}$ & 0.00 & 0.00 & NA & NA & NA \\
\hline & $7: 117308226$ & & Down-stream & $\mathrm{T} / \mathrm{A}$ & 0.52 & 0.00 & 4.271 & $2.91 \times 10^{-5}$ & 0.00 & 0.00 & NA & NA & NA \\
\hline
\end{tabular}


Table C-6. (Continued)

\begin{tabular}{|c|c|c|c|c|c|c|c|c|c|c|c|c|c|}
\hline \multirow[b]{2}{*}{$\begin{array}{c}\text { Gene } \\
(n \text { of SNPs at } \\
P<0.05 / n \text { of } \\
\text { all SNPs) }^{\mathrm{a}}\end{array}$} & \multirow[b]{2}{*}{ Chr:Loc ${ }^{b}$} & \multirow[b]{2}{*}{ RSID } & \multirow[b]{2}{*}{ Function } & \multirow[b]{2}{*}{$\begin{array}{c}\text { Majora } \\
\text { llele/ } \\
\text { minor } \\
\text { allele }\end{array}$} & \multicolumn{4}{|c|}{ Cohort $(n=4217)$} & \multicolumn{4}{|c|}{ Case-control $(n=162)$} & \multirow[b]{2}{*}{$\begin{array}{l}\text { Dire- } \\
\text { ction }^{\mathrm{e}}\end{array}$} \\
\hline & & & & & $\begin{array}{l}\text { MAF\% } \\
\text { in } \\
\text { patients } \\
\text { with AP } \\
(n=95)\end{array}$ & $\begin{array}{l}\text { MAF\% } \\
\text { in } \\
\text { patients } \\
\text { without } \\
\text { AP } \\
(n= \\
3,161)\end{array}$ & $\begin{array}{l}\text { Coeff- } \\
\text { icient }^{c}\end{array}$ & $P^{\mathfrak{c}}$ & $\begin{array}{c}\text { MAF } \\
\% \text { in } \\
\text { cases } \\
(n= \\
47)\end{array}$ & $\begin{array}{l}\text { MAF } \\
\% \text { in } \\
\text { con- } \\
\text { trols } \\
(n= \\
115)\end{array}$ & $\begin{array}{l}\text { Coeff- } \\
\text { icient }^{d}\end{array}$ & $P^{d}$ & \\
\hline \multirow{17}{*}{$\begin{array}{c}C P A 1^{80,81} \\
(1 / 161) \\
P_{69 S S 1^{67-}} \\
(22 / 283)\end{array}$} & $7: 130020234$ & & Upstream & $\mathrm{C} / \mathrm{T}$ & 0.52 & 0.00 & 4.070 & $1.11 \times 10^{-4}$ & 0.00 & 0.00 & NA & NA & NA \\
\hline & $7: 142457184$ & & Upstream & $\mathrm{C} / \mathrm{T}$ & 0.52 & 0.01 & 4.064 & $6.24 \times 10^{-5}$ & 0.00 & 0.00 & NA & NA & NA \\
\hline & $7: 142458285$ & & Intronic & $-/ A$ & 1.79 & 0.28 & 1.926 & $9.29 \times 10^{-3}$ & 1.56 & 0.00 & 16.28 & 0.991 & + \\
\hline & $7: 142458357$ & rs19942214 & Intronic & $\mathrm{C} / \mathrm{T}$ & 0.52 & 0.05 & 2.515 & $1.27 \times 10^{-2}$ & 0.00 & 0.00 & NA & NA & NA \\
\hline & $7: 142458582$ & rs 2688125 & Intronic & $\mathrm{C} / \mathrm{T}$ & 2.81 & 0.93 & 1.208 & $8.99 \times 10^{-3}$ & 0.00 & 2.31 & -16.23 & 0.988 & - \\
\hline & $7: 142458604$ & rs375924181 & Intronic & $\mathrm{T} / \mathrm{G}$ & 1.60 & 0.59 & 1.162 & $4.85 \times 10^{-2}$ & 0.00 & 1.40 & -16.45 & 0.990 & - \\
\hline & $7: 142458638$ & rs369272152 & Intronic & $\mathrm{C} / \mathrm{G}$ & 1.28 & 0.33 & 1.517 & $3.55 \times 10^{-2}$ & 1.11 & 0.00 & 15.47 & 0.986 & + \\
\hline & $7: 142459524$ & & Intronic & $\mathrm{C} / \mathrm{A}$ & 22.08 & 15.56 & 0.464 & $4.82 \times 10^{-2}$ & 16.67 & 20.45 & -0.30 & 0.497 & - \\
\hline & $7: 142459525$ & & Intronic & $\mathrm{A} / \mathrm{G}$ & 22.37 & 15.67 & 0.491 & $3.75 \times 10^{-2}$ & 17.74 & 19.78 & -0.15 & 0.745 & - \\
\hline & $7: 142459543$ & & Intronic & $\mathrm{A} / \mathrm{G}$ & 35.71 & 25.92 & 0.826 & $3.48 \times 10^{-3}$ & 33.33 & 31.55 & 0.18 & 0.697 & + \\
\hline & $7: 142459547$ & & Intronic & $\mathrm{C} / \mathrm{G}$ & 20.39 & 13.84 & 0.522 & $2.81 \times 10^{-2}$ & 12.20 & 19.68 & -0.70 & 0.109 & - \\
\hline & $7: 142459550$ & & Intronic & $\mathrm{G} / \mathrm{A}$ & 6.90 & 6.62 & 0.085 & $7.85 \times 10^{-1}$ & 8.75 & 2.53 & 1.43 & 0.024 & + \\
\hline & $7: 142459558$ & & Intronic & $\mathrm{T} /-$ & 6.47 & 5.30 & 0.263 & $4.17 \times 10^{-1}$ & 8.75 & 2.58 & 1.40 & 0.026 & + \\
\hline & $7: 142459771$ & rs199769221 & Nonsyn & $\mathrm{G} / \mathrm{C}$ & 0.53 & 0.08 & 2.434 & $1.62 \times 10^{-2}$ & 0.00 & 0.00 & NA & NA & NA \\
\hline & $7: 142459778$ & & Syn & $\mathrm{A} / \mathrm{C}$ & 0.53 & 0.04 & 2.602 & $1.01 \times 10^{-2}$ & 0.00 & 0.00 & NA & NA & NA \\
\hline & $7: 142459784$ & & Syn & $\mathrm{C} / \mathrm{T}$ & 0.52 & 0.05 & 2.491 & $1.38 \times 10^{-2}$ & 0.00 & 0.00 & NA & NA & NA \\
\hline & $7: 142459785$ & & Nonsyn & $\mathrm{G} / \mathrm{T}$ & 0.52 & 0.02 & 3.417 & $8.34 \times 10^{-4}$ & 0.00 & 0.00 & NA & NA & NA \\
\hline
\end{tabular}




\section{Table C-6. (Continued)}

\begin{tabular}{|c|c|c|c|c|c|c|c|c|c|c|c|c|c|}
\hline \multirow[b]{2}{*}{$\begin{array}{c}\text { Gene } \\
\text { (n of SNPs at } \\
P<0.05 / n \text { of } \\
\text { all SNPs) }\end{array}$} & \multirow[b]{2}{*}{ Chr:Loc ${ }^{b}$} & \multirow[b]{2}{*}{ RSID } & \multirow[b]{2}{*}{ Function } & \multirow[b]{2}{*}{$\begin{array}{c}\text { Major } \\
\text { allele/ } \\
\text { minor } \\
\text { allele }\end{array}$} & \multicolumn{4}{|c|}{ Cohort $(n=4217)$} & \multicolumn{4}{|c|}{ Case-control $(n=162)$} & \multirow[b]{2}{*}{$\begin{array}{l}\text { Dire- } \\
\text { ction }^{\text {e }}\end{array}$} \\
\hline & & & & & $\begin{array}{l}\text { MAF\% } \\
\text { in } \\
\text { patients } \\
\text { with AP } \\
(n=95)\end{array}$ & $\begin{array}{c}\text { MAF\% } \\
\text { in } \\
\text { patients } \\
\text { without } \\
\text { AP } \\
(\mathrm{n}= \\
3,161)\end{array}$ & $\begin{array}{l}\text { Coeff- } \\
\text { icient }^{c}\end{array}$ & $P^{\mathbf{c}}$ & $\begin{array}{l}\text { MAF\% } \\
\text { in cases } \\
(n=47)\end{array}$ & $\begin{array}{c}\text { MAF\% } \\
\text { in con- } \\
\text { trols } \\
(n= \\
115)\end{array}$ & $\begin{array}{l}\text { Coeff- } \\
\text { icient }^{\mathrm{d}}\end{array}$ & $P^{\mathrm{d}}$ & \\
\hline \multirow{6}{*}{$\begin{array}{c}P_{69,74,162} \\
(22 / 283)\end{array}$} & $7: 142460163$ & rs111626281 & Intronic & $\mathrm{T} / \mathrm{C}$ & 1.03 & 0.10 & 1.938 & $9.30 \times 10^{-3}$ & 0.00 & 0.00 & NA & NA & NA \\
\hline & $7: 142460323$ & & Syn & $\mathrm{C} / \mathrm{T}$ & 0.52 & 0.00 & 3.952 & $1.44 \times 10^{-4}$ & 0.00 & 0.00 & NA & NA & NA \\
\hline & $7: 142460432$ & & Intronic & $\mathrm{C} / \mathrm{A}$ & 2.78 & 0.00 & 2.850 & $1.12 \times 10^{-2}$ & 0.00 & 2.50 & -17.58 & 0.994 & - \\
\hline & $7: 142460495$ & & Intronic & $\mathrm{A} / \mathrm{C}$ & 41.28 & 41.98 & -0.130 & $6.49 \times 10^{-1}$ & 40.24 & 45.71 & -1.07 & 0.046 & + \\
\hline & $7: 142460503$ & & Intronic & $\mathrm{T} / \mathrm{C}$ & 38.10 & 37.95 & 0.021 & $9.36 \times 10^{-1}$ & 36.59 & 43.43 & -0.95 & 0.045 & - \\
\hline & $7: 142460556$ & rs374121411 & Intronic & $\mathrm{C} / \mathrm{T}$ & 17.11 & 18.90 & -0.151 & $5.41 \times 10^{-1}$ & 28.57 & 17.82 & 0.95 & 0.025 & - \\
\hline \multirow{8}{*}{$\begin{array}{c}P R S S 2^{66,69} \\
(8 / 43)\end{array}$} & $7: 142479912$ & rs248489 & Unknown & $\mathrm{C} / \mathrm{G}$ & 46.28 & 41.94 & 0.811 & $4.46 \times 10^{-2}$ & 38.89 & 43.75 & -0.57 & 0.252 & - \\
\hline & $7: 142479917$ & rs36959197 & Unknown & $\mathrm{C} / \mathrm{T}$ & 46.77 & 41.47 & 1.059 & $1.43 \times 10^{-2}$ & 37.78 & 43.36 & -0.64 & 0.187 & - \\
\hline & $7: 142479940$ & rs58649169 & Unknown & $\mathrm{C} / \mathrm{T}$ & 45.83 & 40.96 & 0.742 & $3.66 \times 10^{-2}$ & 38.30 & 43.04 & -0.53 & 0.267 & - \\
\hline & $7: 142479954$ & rs21779716 & Unknown & $\mathrm{C} / \mathrm{T}$ & 45.83 & 40.74 & 0.839 & $2.62 \times 10^{-2}$ & 38.30 & 43.04 & -0.53 & 0.267 & - \\
\hline & $7: 142480002$ & rs199512312 & Unknown & $\mathrm{C} / \mathrm{A}$ & 45.88 & 40.63 & 0.860 & $2.37 \times 10^{-2}$ & 38.30 & 43.04 & -0.53 & 0.267 & - \\
\hline & $7: 142480012$ & rs56146961 & Unknown & $\mathrm{C} / \mathrm{T}$ & 44.85 & 40.07 & 0.699 & $4.16 \times 10^{-2}$ & 38.30 & 42.61 & -0.44 & 0.354 & - \\
\hline & $7: 142480053$ & rs 2815151 & Unknown & $\mathrm{G} / \mathrm{C}$ & 4.40 & 2.39 & 0.776 & $3.73 \times 10^{-2}$ & 0.00 & 3.92 & -16.93 & 0.990 & - \\
\hline & $7: 142480063$ & rs2216921 & Unknown & $\mathrm{C} / \mathrm{A}$ & 1.11 & 0.13 & 2.280 & $1.50 \times 10^{-3}$ & 0.00 & 0.47 & -14.24 & 0.987 & - \\
\hline $\begin{array}{c}C L D N 2^{66,69} \\
(1 / 67)\end{array}$ & $X: 106163851$ & & Intronic & $\mathrm{T} / \mathrm{A}$ & 0.53 & 0.00 & 3.885 & $1.45 \times 10^{-4}$ & 0.00 & 0.00 & NA & NA & NA \\
\hline
\end{tabular}




\section{Table C-6. (Continued)}

${ }^{\mathrm{a}}$ (Number of SNPs at the $P<0.05$ level)/(number of SNPs identified in the gene). ${ }^{\mathrm{b}}$ Physical location of SNP based on Feb 2009 human genome assembly (hg19). ${ }^{\mathrm{C}}$ Coefficient and P value of minor alleles were estimated using Cox proportional hazard model. ${ }^{\mathrm{d} C o e f f i c i e n t}$ and $\mathrm{P}$ value of minor alleles were estimated using logistic regression model. $\mathrm{P}$ values less than 0.05 were highlighted. ${ }^{\mathrm{e}}$ The SNP goes in the same (+) or different (-) direction in the cohort and the case-control group, or is absent (NA) in the case-control group. MAF, minor allele frequency. RSID, reference SNP identification number. Syn, synonymous.

Nonsyn, nonsynonymous. NA, not applicable. 


\section{VITA}

Chengcheng Liu was born 1985 in Dalian, China. She received her B.S. degree in Chemistry and Biology at Tsinghua University in 2008. She joined in the Integrated

Program of Biomedical Sciences at University of Tennessee Health Science Center in 2009, and anticipated to complete her Ph.D. study in 2015. 